Florida International University FIU Digital Commons

\title{
Microstructure and Mechanical Properties of Nanofiller Reinforced Tantalum-Niobium Carbide Formed by Spark Plasma Sintering
}

Christopher Charles Rudolf

Florida International University, crudo003@fiu.edu

DOI: 10.25148 /etd.FIDC000728

Follow this and additional works at: https:// digitalcommons.fiu.edu/etd

Part of the Ceramic Materials Commons, Mechanics of Materials Commons, and the Nanoscience and Nanotechnology Commons

\section{Recommended Citation}

Rudolf, Christopher Charles, "Microstructure and Mechanical Properties of Nanofiller Reinforced Tantalum-Niobium Carbide Formed by Spark Plasma Sintering" (2016). FIU Electronic Theses and Dissertations. 2596.

https://digitalcommons.fiu.edu/etd/2596 


\title{
FLORIDA INTERNATIONAL UNIVERSITY
}

Miami, Florida

MICROSTRUCTURE AND MECHANICAL PROPERTIES OF NANOFILLER

REINFORCED TANTALUM-NIOBIUM CARBIDE FORMED BY SPARK PLASMA

SINTERING

\author{
A dissertation submitted in partial fulfillment of \\ the requirements for the degree of \\ DOCTOR OF PHILOSOPHY \\ in \\ MECHANICAL ENGINEERING \\ by
}

Christopher Rudolf

2016 
To: Interim Dean Ranu Jung

College of Engineering and Computing

This dissertation, written by Christopher Rudolf, and entitled Microstructure and Mechanical Properties of Nanofiller Reinforced Tantalum-Niobium Carbide Formed by Spark Plasma Sintering, having been approved in respect to style and intellectual content, is referred to you for judgment.

We have read this dissertation and recommend that it be approved.

Zhe Cheng

Wenzhi Li

Baburaj Eranezhuth

Benjamin Boesl,Co-Major Professor

Arvind Agarwal,Co-Major Professor

Date of Defense: May 26, 2016

The dissertation of Christopher Rudolf is approved.

Interim Dean Ranu Jung
College of Engineering and Computing

Florida International University, 2016 


\section{DEDICATION}

I dedicate this dissertation to my wife and my parents. Without their support and encouragement I would not have had the opportunity to expand my education. 


\section{ACKNOWLEDGMENTS}

I sincerely thank my advisors, Dr. Benjamin Boesl and Dr. Arvind Agarwal for their extensive support through my entire time at Florida International University. Without your approaching me to pursue my doctorate when I finished my master's degree, this would have never come to fruition. The guidance you have given me has been invaluable. Whenever I found my research progress slowing, you were both there to suggest new directions to re-energize it. I thank you both for your patience when I did not immediately understand something, and for taking the time to sit down with me to figure it out.

I extend my thanks to all of my committee members including Dr. Zhe Cheng, Dr. Wenzhi Li, and Dr. Baburaj Eranezhuth. In addition to thanking them for agreeing to serve on my dissertation committee, I am grateful for the support and encouragement I have received throughout my research.

I would like to acknowledge the generous support of the Minority Leaders/Research Collaboration Program with Clarkson Aerospace and UTC and those involved including Dr. Baburaj Eranezhuth, Dr. R. Douglas Hutchens, Mr. Tobey Cordell, and Ms. DeAnna Brown.

I am thankful for the support afforded to me by a research grant from the Air Force Research Laboratory (Contract FA8560-13-C-5800, AFRL/RXOP Authority to Proceed) and those individuals involved including technical coordinator, Dr. Lawrence Matson and program manager, Ms. Asheley Blackford. 
I would like to acknowledge Florida International University and the University Graduate School. Thank you for supporting me through a Dissertation Year Fellowship (DYF) award.

I am grateful for the support of the Advanced Materials Engineering Research Institute (AMERI) at FIU for providing excellent research facilities and all those who have helped me with my experimentation, especially Dr. Alex Franco.

I would like to thank all of the staff of the Mechanical and Materials Engineering Department at FIU, especially Dr. Cesar Levy for his guidance in completing my coursework.

I am extremely thankful for all of my PFL groupmates - especially Cheng Zhang who has been present whenever I needed to run SPS or whenever I had any questions.

Last but not least, I want to thank those closest to me who have supported me through this endeavor. To my parents, thank you for always being by my side. The support and encouragement you have given me has been incredible, and without it I would have never gotten to where I am today. To my wife, Erica, there has never been a time over the six years we have been together that you did not given me your full support and love and for that I cannot thank you enough. I am grateful you have pushed me to continue my education. Thank you for always offering encouragement even at the most stressful of times. 


\begin{abstract}
OF THE DISSERTATION
MICROSTRUCTURE AND MECHANICAL PROPERTIES OF NANOFILLER REINFORCED TANTALUM-NIOBIUM CARBIDE FORMED BY SPARK PLASMA SINTERING
\end{abstract}

by

Christopher Rudolf

Florida International University, 2016

Miami, Florida

Professor Benjamin Boesl, Co-Major Professor

Professor Arvind Agarwal, Co-Major Professor

Ultra high temperature ceramics (UHTC) are candidate materials for high temperature applications such as leading edges for hypersonic flight vehicles, thermal protection systems for spacecraft, and rocket nozzle throat inserts due to their extremely high melting points. Tantalum and Niobium Carbide ( $\mathrm{TaC}$ and $\mathrm{NbC})$, with melting points of $3950^{\circ} \mathrm{C}$ and $3600^{\circ} \mathrm{C}$, respectively, have high resistivity to chemical attack, making them ideal candidates for the harsh environments UHTCs are to be used in. The major setbacks to the implementation of UHTC materials for these applications are the difficulty in consolidating to full density as well as their low fracture toughness. In this study, small amounts of sintering additive were used to enhance the densification and Graphene Nanoplatelets (GNP) were dispersed in the ceramic composites to enhance the fracture toughness. While the mechanisms of toughening of GNP addition to ceramics have been previously documented, this study focused on the anisotropy of the mechanisms. Spark plasma sintering was used to consolidate both bulk GNP pellets and 
near full relative density $\mathrm{TaC}-\mathrm{NbC}$ ceramic composites with the addition of both sintering aid and GNP and resulted in an aligned GNP orientation perpendicular to the SPS pressing axis that allowed the anisotropy to be studied. In situ high load indentation was performed that allowed real time viewing of the deformation mechanisms for enhanced analysis. The total energy dissipation when indenting the bulk GNP pellet in the in-plane GNP direction was found to be $270 \%$ greater than in the out-of-plane orientation due to the resulting deformation mechanisms that occurred. In GNP reinforced TaC-NbC composites, the projected residual damaged area as a result of indentation was $89 \%$ greater when indenting on the surface of the sintered compact (out-of-plane GNP orientation) than when indenting in the orthogonal direction (in-plane GNP orientation) which is further evidence to the anisotropy of the GNP reinforcement. 


\section{TABLE OF CONTENTS}

CHAPTER

PAGE

Chapter I: Introduction.................................................................................... 1

1. UHTCs and Their High Temperature Applications - Advantages and Limitations ... 1

1.2 Graphene Nanoplatelet Reinforcement in Composites ...................................... 7

1.3 Objectives of the Current Research............................................................. 7

1.3.1 Investigate the Toughening Mechanisms of GNP ..................................... 8

1.3.2 Achieve Full Relative Densification with a Refined Microstructure ................ 8

1.3.3 Reinforce the Fully Dense Ceramics with GNP for Improved Toughening ...... 9

Chapter II: Literature Review .............................................................................. 10

2.1 Possible UHTC Materials and Why TaC-NbC is Being Investigated ................... 10

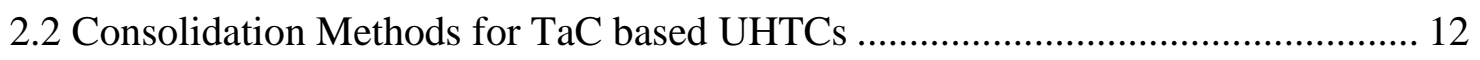

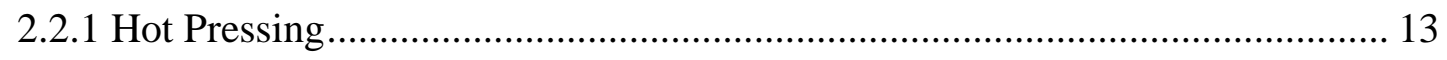

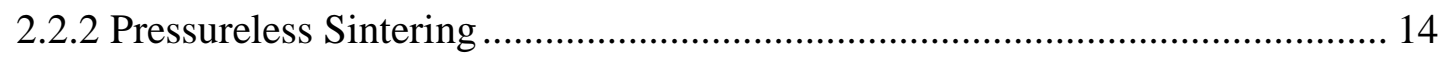

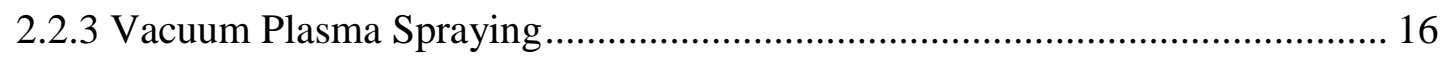

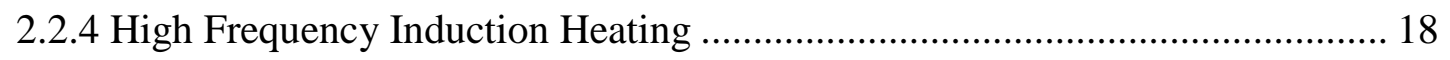

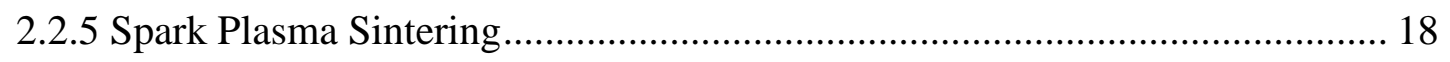

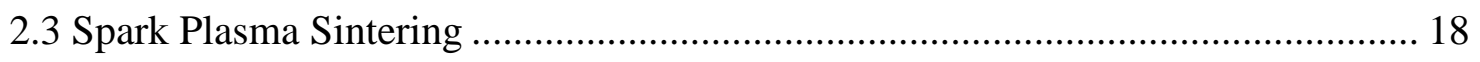

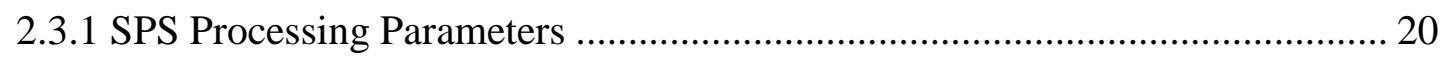

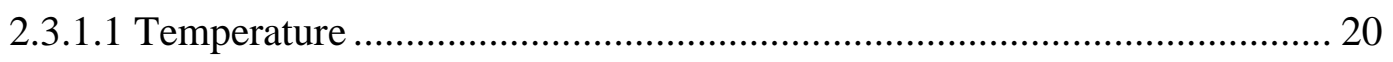

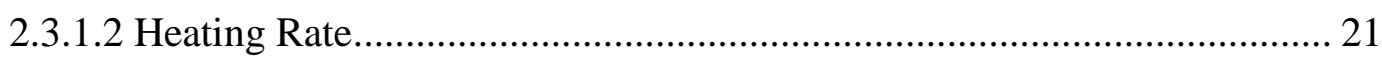

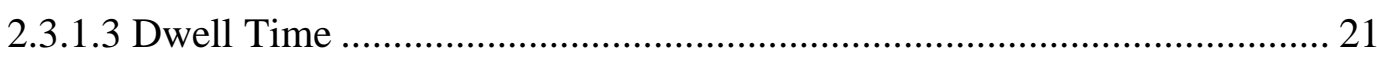

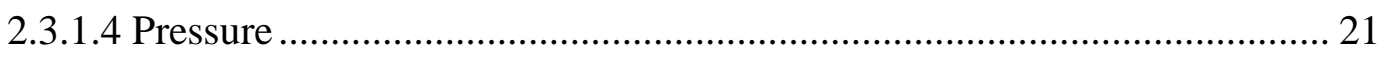

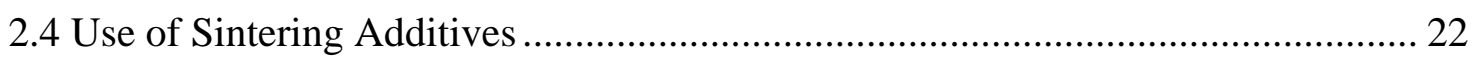

2.5 Materials for Reinforcement in UHTC Composites ........................................... 25

2.5.1 Ceramic Reinforcement to UHTC Composites ............................................. 26

2.5.2 Carbon Nanotube Reinforcement of UHTC Composites ................................ 30

2.5.3 Graphene and Graphene Nanoplatelet Reinforcement of Composites ............. 33

2.6 Reinforcement Mechanisms of GNP in Composites............................................ 34

2.6.1 Regime 1: Property Changes During Processing ......................................... 35

2.6.2 Regime 2: Increased Load Capacity Prior to Initial Crack Propagation .......... 36

2.6.3 Regime 3: Crack Propagation Suppression Mechanisms ............................... 40 


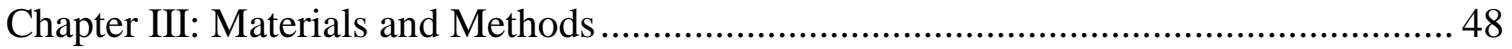

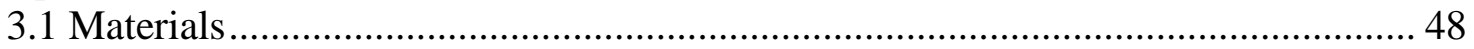

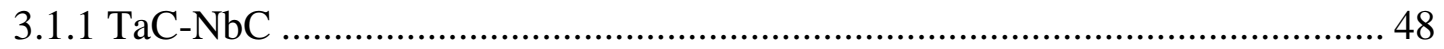

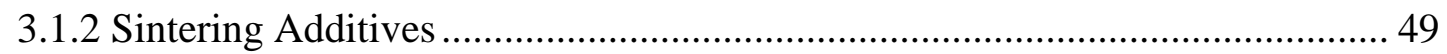

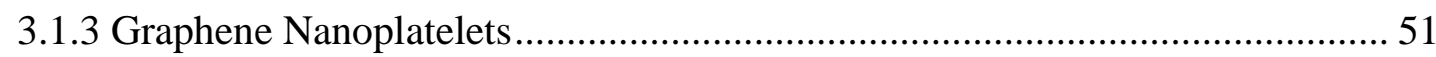

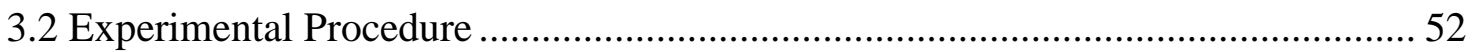

3.2.1 Powder Preparation Methods..................................................................... 52

3.2.2 Consolidation by Spark Plasma Sintering ................................................... 54

3.2.2.1 Initial Evaluation of Processing Parameters .......................................... 56

3.2.2.2 Evaluation of SPS Densification Stages .......................................... 57

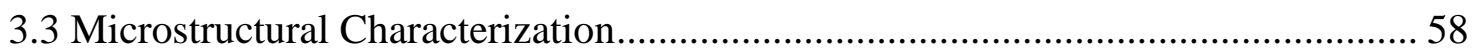

3.4 Evaluation of Mechanical Properties ............................................................... 59

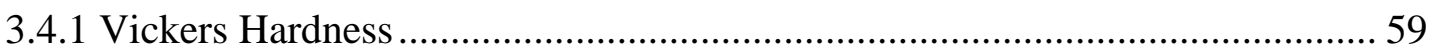

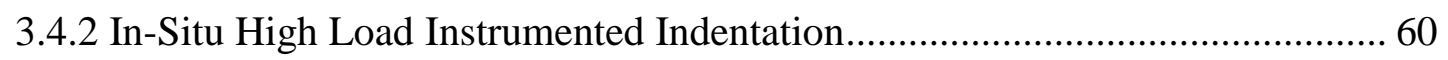

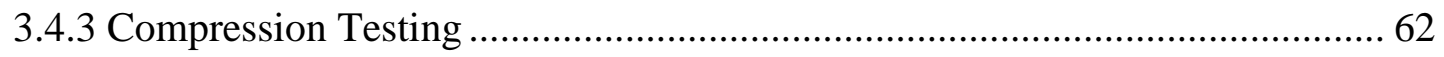

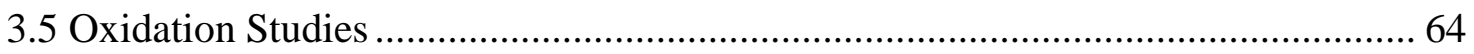

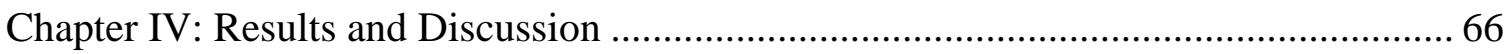

4.1 Intrinsic Deformation Mechanisms of GNP and Their Energy Dissipation

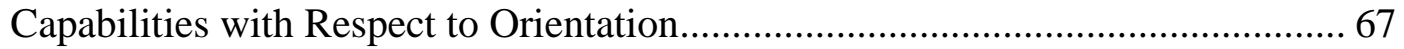

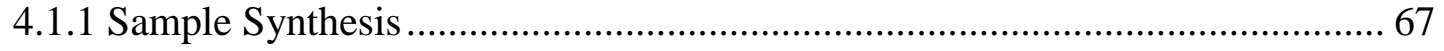

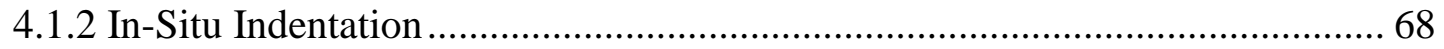

4.1.3 Conclusions Based on the Study of Bulk GNP Deformation Mechanism

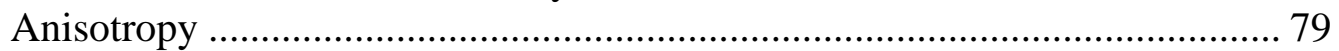

4.2 Powder Processing and Sintering Parameters for the Ceramic Composites .......... 79

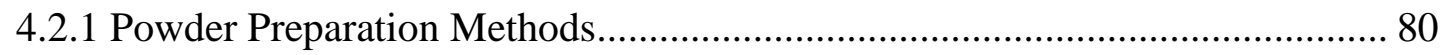

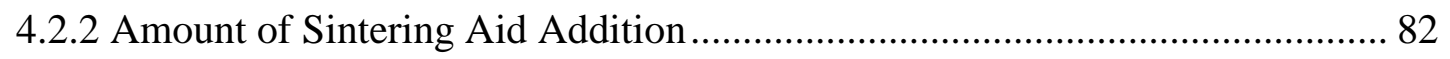

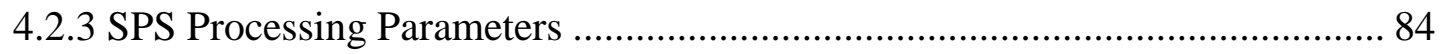

4.3 Use of Sintering Additives for Full Relative Densification ................................ 86

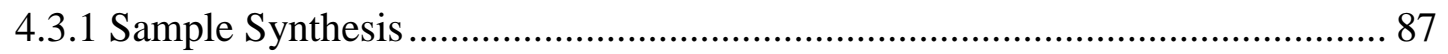

4.3.2 Density, Microstructure, and Secondary Phase Formations ........................... 87

4.3.3 Densification Process and Mechanisms of Sintering .................................... 96

4.3.4 High Load Instrumented Indentation....................................................... 99 
4.3.5 Conclusions Based on the Use of Sintering Additives ................................... 104

4.4 Addition of GNP to Fully Dense TaC-NbC Composites ...................................... 105

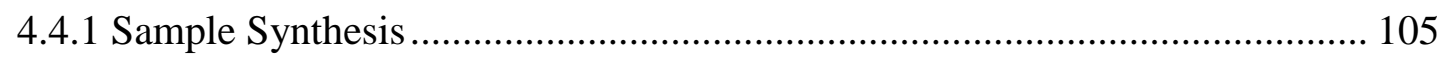

4.4.2 Density, Microstructure, and Secondary Phase Analysis ................................ 106

4.4.2.1 Comparison to Compositions without GNP Addition ............................. 114

4.4.3 Mechanical Properties by Indentation ......................................................... 116

4.4.3.1 Comparison to Compositions without GNP Reinforcement.................... 130

4.4.4 Conclusions with Respect to GNP Reinforcement of the Ceramic

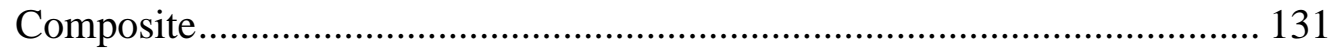

4.5 Compression Testing and Interfacial Stresses.................................................... 131

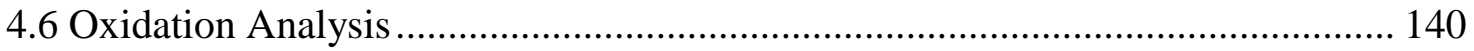

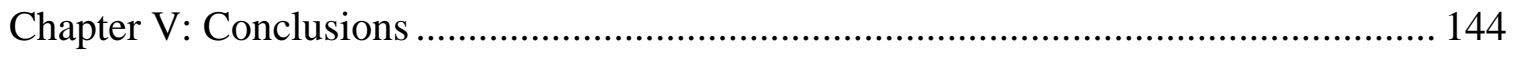

Chapter VI: Recommendations for Future Work...................................................... 148

6.1 Analysis of SiC Reaction between Si and GNP ............................................ 148

6.2 Transmission Electron Microscopy (TEM) Analysis for a Greater Understanding of the Stress Transfer between the Ceramic Matrix and the

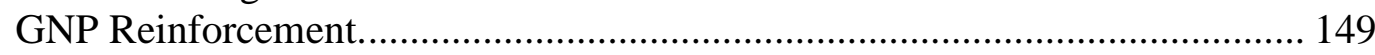

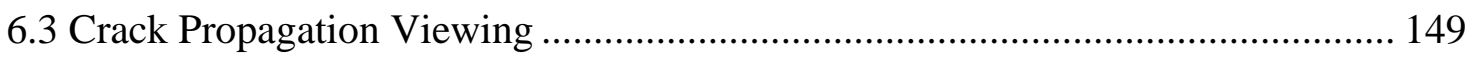

6.4 Investigation into Alternative Ceramic Stoichiometries and Compositions ......... 150

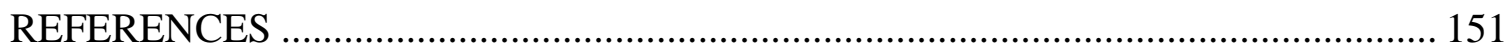

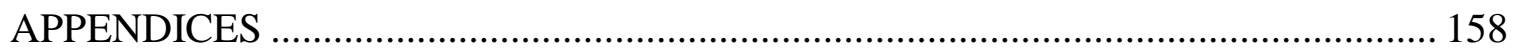

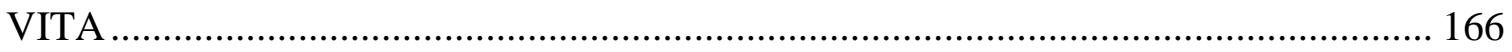




\section{LIST OF TABLES}

TABLE

PAGE

Table 1: Property comparison of $\mathrm{TaC}$ and $\mathrm{NbC}$.

Table 2: Reaction initiation temperatures of compositions after being coated with molten $\mathrm{Al}_{2} \mathrm{O}_{3}$ in solid Aluminum burning rocket nozzles [25]. 12

Table 3: Comparison of consolidation methods for TaC based UHTCs. ....................... 13

Table 4: Properties of TaC and TaC-4 wt.\% CNT compacts prepared by SPS [49]....... 31

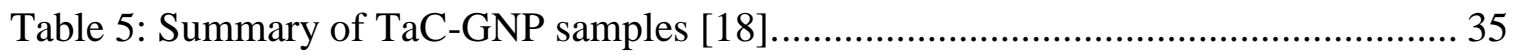

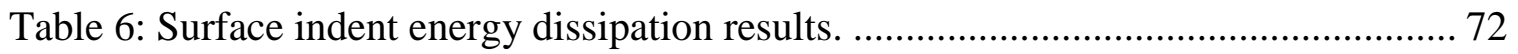

Table 7: Orthogonal indent energy dissipation results. ............................................ 75

Table 8: GNP deformation mechanisms in order of energy dissipation capabilities........ 78

Table 9: Evaluation of SPS processing parameters. ............................................. 85

Table 10: Properties of sintered compacts of $\mathrm{TaC}-\mathrm{NbC}$ with and without sintering additives prepared by SPS at $1850^{\circ} \mathrm{C}$ and a heating rate of $100^{\circ} \mathrm{C} / \mathrm{min}$......... 88

Table 11: Indentation properties of spark plasma sintered $\mathrm{TaC}-\mathrm{NbC}$ compacts with and without sintering additives....

Table 12: Properties of sintered compacts of TaC-NbC-3GNP with and without sintering additives prepared by SPS at $1850^{\circ} \mathrm{C}$ and $60 \mathrm{MPa}$ with a heating rate of $100^{\circ} \mathrm{C} / \mathrm{min}$.

Table 13: Comparison of microstructures and SPS currents of the various ceramic compositions.

Table 14: Indentation properties of spark plasma sintered TaC-NbC compacts.

Table 15: Fracture strength and energy results from diametral compression testing.

Table 16: Values used in calculating the interfacial shear and axial stresses in the varying compositions along with the resulting stresses.

Table 17: Interfacial behavior possibilities between a GNP and the ceramic matrix. 


\section{LIST OF FIGURES}

FIGURE

PAGE

Figure 1: a) Leading edges on hypersonic flight vehicles [3]. b) Heat experienced on ceramic tiles during re-entry into the atmosphere by the space shuttle [4]. c) Schematic showing the components of a rocket nozzle [5].

Figure 2: Flame temperatures of various rocket fuels along with the melting points of various high temperature materials [6] ......................................... 3

Figure 3: Melting point versus density of select UHTC materials [6].......................... 4

Figure 4: Fracture surface of a spark plasma sintered sample with a magnified view showing a porous region. .......................................................... 5

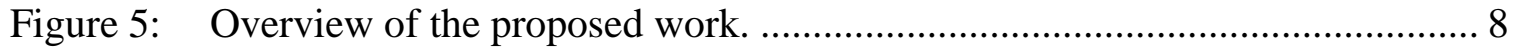

Figure 6: Microstructures of tantalum carbide hot pressed without additives at

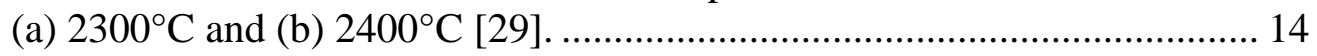

Figure 7: Scanning electron micrographs of the morphologies of the fracture surfaces of the tantalum carbide ceramics. (a) SB22, (b) SB23, (c) SB24, (d) SC22, (e) SC23, and (f) SC24 [32].

Figure 8: Vacuum plasma-sprayed TaC cylinder $50 \mathrm{~mm}$ diameter, $100 \mathrm{~mm}$ height, and $1.5 \mathrm{~mm}$ thick [34].

Figure 9: Schematic of the Spark Plasma Sintering System. 19

Figure 10: SEM micrographs of fracture surfaces of TaC samples prepared by SPS at (a) $1850^{\circ} \mathrm{C}$ and $100 \mathrm{MPa}$, (b) $1850^{\circ} \mathrm{C}$ and $255 \mathrm{MPa}$, and (c) $1850^{\circ} \mathrm{C}$ and $363 \mathrm{MPa}[37]$.

Figure 11: Secondary electron images of the microstructures of the consolidated TaC materials with (a) 5 mol.\% Al, (b) 5 mol.\% Cu, (c) 5 mol.\% Ag, and (d) 5 mol.\% Au additions [44].

Figure 12: (a) SEM micrograph of the fracture surface of TaC-BC-363 sample indicating the presence of $\mathrm{Carbon} / \mathrm{TaB}_{2}$ at $\mathrm{TaC}$ grain boundaries, (b) TEM image of TaC-BC-363 sample showing the presence of carbon flakes between $\mathrm{TaC}$ grains [37].

Figure 13: (a) Polished section parallel to the direction of applied pressure, (b) Polished section normal to the direction of applied pressure [47]. 
Figure 14: Examples of crack path in (a) baseline material, (b) Z10w and (c) Z20w showing increasing tortuosity of the crack profile with the increase of whisker amount [47].

Figure 15: SEM micrograph of fracture surfaces for (a) TaC, (b) TaC-LC, and (c) TaC-SC [51].

Figure 16: SEM images of fracture surface of GPL-reinforced $\mathrm{Al}_{2} \mathrm{O}_{3}$ composite showing the toughening and reinforcing mechanisms [17].

Figure 17: SEM images showing (a) delamination of a GNS-HA interface and (b) a GNS rupture in a GNS/HA composite [19]. 38

Figure 18: GNP toughening mechanisms in TaC: a) GNP bending and kinking, b) GNP sliding [18].

Figure 19: Toughening mechanisms in $\mathrm{GPL}_{-} \mathrm{Si}_{3} \mathrm{~N}_{4}$ nanocomposites. (a) Microhardness testing resulting in the creation of radial cracks stemming from the microhardness indent (inset image). Closer examination of the radial cracks reveals GPL bridging the crack at several locations, two of which are shown in this high-resolution SEM image. (b) Further examination of the radial cracks indicates that they follow a tortuous crack propagation path. (c) Fracture surface of the bulk sample indicates the presence of three-dimensional toughening mechanisms for the GPL-Si ${ }_{3} \mathrm{~N}_{4}$ nanocomposite [60].

Figure 20: Toughening mechanisms in composites. (a) Crack deflection and slow down of the crack propagation at GPLs (fracture line), (b) crack deflection parallel and perpendicular to the fracture surface, (c) crack bridging by FPLs on the fracture line with the plane of sheet nearly parallel to the plane of the polished surface, (d) crack bridging with a GPL on the fracture surface, with the plane of the sheet perpendicular to the plane of the fracture surface [61].

Figure 21: Simplified schematics of the measuring geometry to illustrate the possible conducting paths for the orientations (a) perpendicular and (b) parallel with respect to the SPS pressing axis [64]. 45

Figure 22: Examples of 3D unit cell models of graphene reinforced composites:

(a) Aligned exfoliated model, (b) Random exfoliated model [65]. 46

Figure 23: Crack morphology in an aligned and random model [65] 46

Figure 24: SEM image of the as-received TaC-NbC powder 49

Figure 25: SEM images of $\mathrm{B}_{4} \mathrm{C}$ and $\mathrm{Si}$ powders. 50 
Figure 26: (a) SEM and (b) TEM images of as-received Graphene Nanoplatelets. ....... 51

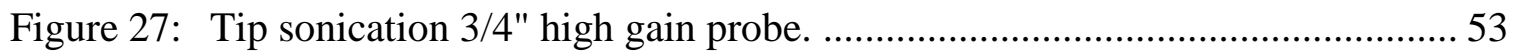

Figure 28: Thermal Technologies Model 10-4 SPS machine installed in the Plasma Forming Lab at FIU. ............................................................. 54

Figure 29: Schematic of the graphite punches and die assembly................................55

Figure 30: Representative plot of the SPS processing parameter outputs...................... 56

Figure 31: Instantaneous relative densities during the spark plasma sintering

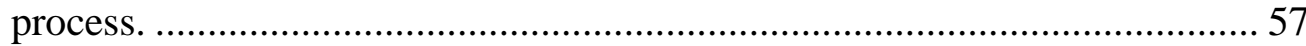

Figure 32: (a) Schematic of the micro-load testing frame used for in-situ instrumented indentation. (b) View of the sample situated across from the indentation tip.

Figure 33: Image of load frame inside the scanning electron microscope for in situ testing.

Figure 34: SEM images of (A) the fracture surface and (B) the top surface of spark plasma sintered bulk GNP compacts. (C) Schematic of the surface and orthogonal indentation directions.

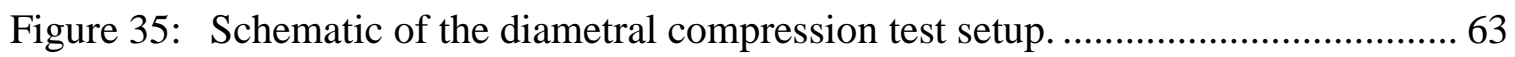

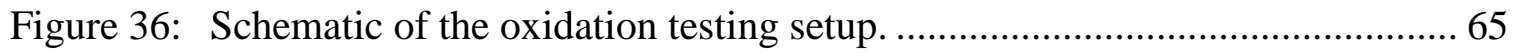

Figure 37: SEM images of A) fracture surface and B) top surface of spark plasma sintered bulk GNP pellet.

Figure 38: Schematic showing the surface and orthogonal indentation orientations.

Figure 39: Surface indent Load-Displacement and Work-Displacement curves and images of deformation occurrences.

Figure 40: Orthogonal indent Load-Displacement and Work-Displacement curves and images of deformation occurrences.

Figure 41: SEM images of A) overall residual indentation and magnified images of deformation mechanisms corresponding to the locations marked in image (A) as a result of the surface indentation: B) sheet sliding with arrows marking wrinkling, C) bending and kinking, and D) sheet pullout. 
Figure 42: SEM images of A) overall residual indentation and magnified images of deformation mechanisms corresponding to the locations marked in image (A) as a result of orthogonal indentation: B) separation and cracking, C) crack propagation, and D) bending during indentation.

Figure 43: SEM images of TaC-NbC-7 vol.\% Si-3 vol.\% GNP dispersed by a) bath sonication, b) ball milling, c) blending, and d) tip sonication.

Figure 44: Relative density measurements using 5 and 10 vol.\% sintering aid addition.

Figure 45: Elastic modulus measurements using 5 and 10 vol. $\%$ sintering aid addition.

Figure 46: Hardness measurements using 5 and 10 vol.\% sintering aid addition.

Figure 47: Instantaneous relative densities during the spark plasma sintering process.

Figure 48: SEM images of the fracture surfaces of TaC-NbC-5 vol.\% Si addition sintered with a 10 minute and 3 minute dwell time.

Figure 49: SEM micrographs of the fracture surfaces of TaC-NbC, TaC-NbC$5 \mathrm{~B}_{4} \mathrm{C}$, and $\mathrm{TaC}-\mathrm{NbC}-5 \mathrm{Si}$ prepared by SPS.

Figure 50: Representative cross-sectional image of sintered TaC-NbC-5 vol.\% Si showing dense microstructure and grains using focused ion beam after milling.

Figure 51: X-ray diffraction patterns for $\mathrm{TaC}-\mathrm{NbC}$ composites with and without sintering additives after spark plasma sintering.

Figure 52: SPS processing and relative densification of TaC-NbC with 5 vol.\% $\mathrm{B}_{4} \mathrm{C}$ addition as a function of the sintering process. Stages of sintering (marked on figure as I-VI) are the following: (I) Initial increase in SPS ram pressure, (II) continued ramping of temperature, (III) rapid densification begins accompanied with an increase in chamber pressure, (IV) rapid densification, (V and VI) final densification.

Figure 53: SPS processing and relative densification of TaC-NbC with 5 vol.\% $\mathrm{Si}$ addition as a function of the sintering process. Stages of sintering (marked on figure as I-VI) are the following: (I) Initial increase in SPS ram pressure, (II) continued ramping of temperature, (III) rapid densification begins, (IV) rapid densification, (V and VI) final densification. 
Figure 54: High load instrumented indentation load-displacement curves for $\mathrm{TaC}-\mathrm{NbC}$ with sintering additives.

Figure 55: (a) SEM image of indentation impression on TaC-NbC, (b) zoomed in SEM image of area marked on (a) showing cracks protruding from indentation, and (c) a schematic of the cracks as a result of indentation with shaded projected residual damaged area.

Figure 56: Map of projected residual damage resulting from high load instrumented indentation for spark plasma sintered $\mathrm{TaC}-\mathrm{NbC}$ with and without sintering additives.

Figure 57: SEM micrographs of the fractured surfaces of TaC-NbC-3GNP: a) low magnification image showing alignment of the GNP, b) high magnification image showing interaction between GNP and matrix grains

Figure 58: SEM micrographs of the fractured surfaces of TaC-NbC-5B ${ }_{4} \mathrm{C}-3 \mathrm{GNP}$ : a) low magnification image showing alignment of the GNP, b) high magnification image showing interaction between GNP and matrix grains

Figure 59: SEM migrographs of the fractured surfaces of TaC-NbC-5Si-3GNP sample showing a) low magnification view, b) magnified view of the area marked in (a), and c) magnified view of the area marked in (b) with arrows pointing to elongation of $\mathrm{SiC}$ grains.

Figure 60: X-ray diffraction patterns for TaC-NbC-3GNP composites with and without sintering additives after spark plasma sintering.

Figure 61: Plots of the current outputs during SPS processing for the various ceramic compositions.

Figure 62: Schematic of indentation directions with respect to the GNP orientation.

Figure 63: High load instrumented indentation load-displacement curves for the TaC-NbC-5B ${ }_{4} \mathrm{C}-3 \mathrm{GNP}$ composite in both the surface and orthogonal directions. Ranges of supplementary videos marked on curves.

Figure 64: High load instrumented indentation load-displacement curves for the TaC-NbC-5Si-3GNP composite in both the surface and orthogonal directions

Figure 65: a) SEM image of indentation impression on TaC-NbC-3GNP, b) zoomed in SEM image of area marked on (a) showing cracks 
protruding from indentation, and c) a schematic of the cracks as a result of indentation with shaded projected residual damaged area.

Figure 66: Map of projected residual damage resulting from high load indentation in the surface direction for spark plasma sintered $\mathrm{TaC}$ $\mathrm{NbC}$ composites.

Figure 67: Map of projected residual damage resulting from high load instrumented indentation in the orthogonal direction for spark plasma sintered $\mathrm{TaC}-\mathrm{NbC}$ composites.

Figure 68: Image stills taken from the real-time indentation video of indentation performed in the surface orientation direction for the TaC-NbC-5B 4 3GNP compact.

Figure 69: Image stills taken from the real-time indentation video of indentation performed in the orthogonal orientation direction for the $\mathrm{TaC}-\mathrm{NbC}$ $5 \mathrm{~B}_{4} \mathrm{C}-3 \mathrm{GNP}$ compact.

Figure 70: SEM images of intrinsic toughening mechanisms of GNP: a) crack bridging in the orthogonal direction, b) crack deflection, c) crack bridging in the surface direction, d) crack arrest, and e) sheet sliding.

Figure 71: Load-displacement curves resulting from diametral compression testing.

Figure 72: Post compression test SEM imaging of the fracture surface of TaCNbC-3GNP.

Figure 73: Post compression test SEM imaging of the fracture surface of TaC$\mathrm{NbC}-5 \mathrm{~B}_{4} \mathrm{C}-3 \mathrm{GNP}$.

Figure 74: Post compression test SEM imaging of the fracture surface of TaC$\mathrm{NbC}-5 \mathrm{Si}-3 \mathrm{GNP}$.

Figure 75: Schematic of GNP in a ceramic matrix used for the stress-transfer equations.

Figure 76: Temperature profile during oxidation testing measured by thermocouple in contact with back side of sample.

Figure 77: SEM micrograph of the front side oxidation layer surface of the TaCNbC-5 vol.\% Si sample.

Figure 78: SEM micrograph of the front side oxidation layer surface of the TaCNbC-5 vol.\% Si-3 vol.\% GNP sample. 


\section{Chapter I: Introduction}

The aim of this research is to synthesize Graphene Nanoplatelet (GNP) reinforced ultra high temperature ceramic (UHTC) composites for use in extreme environment applications. The intention is to develop a composite that overcomes the existing limitations and disadvantages of UHTCs in terms of low fracture toughness and high oxidation susceptibility. An in-depth analysis of the intrinsic deformation mechanisms of GNP as both a bulk material as well as dispersed throughout a composite is conducted insitu to understand the fundamental toughening benefits.

\section{UHTCs and Their High Temperature Applications - Advantages and Limitations}

Ultra high temperature ceramics (UHTC) are candidate materials for high temperature applications such as leading edges for hypersonic flight vehicles (Figure 1a), thermal protection systems for spacecraft (Figure 1b), and rocket nozzle throat inserts (Figure 1c) due to their extremely high melting points and resistivity to chemical attack. Sharp wing leading edges help reduce the drag, enhance maneuverability and performance, and improve safety due to an increased cross-range capability of flight vehicles [1]. The challenge with sharp wing leading edges is that the convective heating to the surface increases as the edge radius decreases. The surface temperature on the stagnation region of the leading edges can potentially exceed $2000^{\circ} \mathrm{C}$ [2]. 

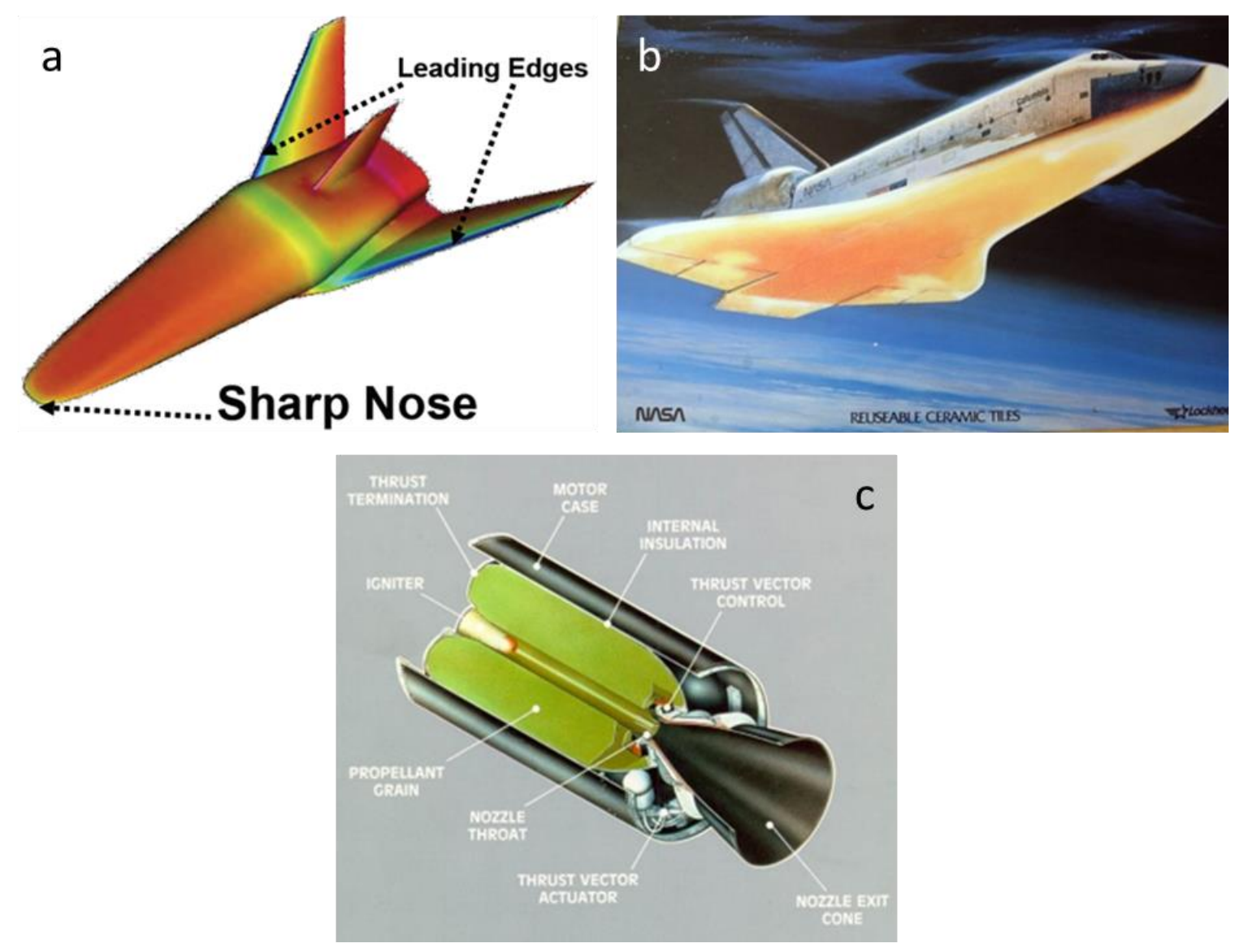

Figure 1: a) Leading edges on hypersonic flight vehicles [3]. b) Heat experienced on ceramic tiles during re-entry into the atmosphere by the space shuttle [4]. c) Schematic showing the components of a rocket nozzle [5].

Thermal protection systems of spacecraft mainly experience convective heating during re-entry; however sufficient energy in the shock layer dissociates air molecules and provides the potential for additional heating. Rocket nozzle throat inserts experience the full flame temperatures of the fuel being used. Figure 2 shows the flame temperatures of various rocket fuels along with the melting points of certain high temperature materials. As can be seen, $\mathrm{TaC}$ and $\mathrm{HfC}$ are both viable options since their melting temperatures exceed the flame temperatures. 


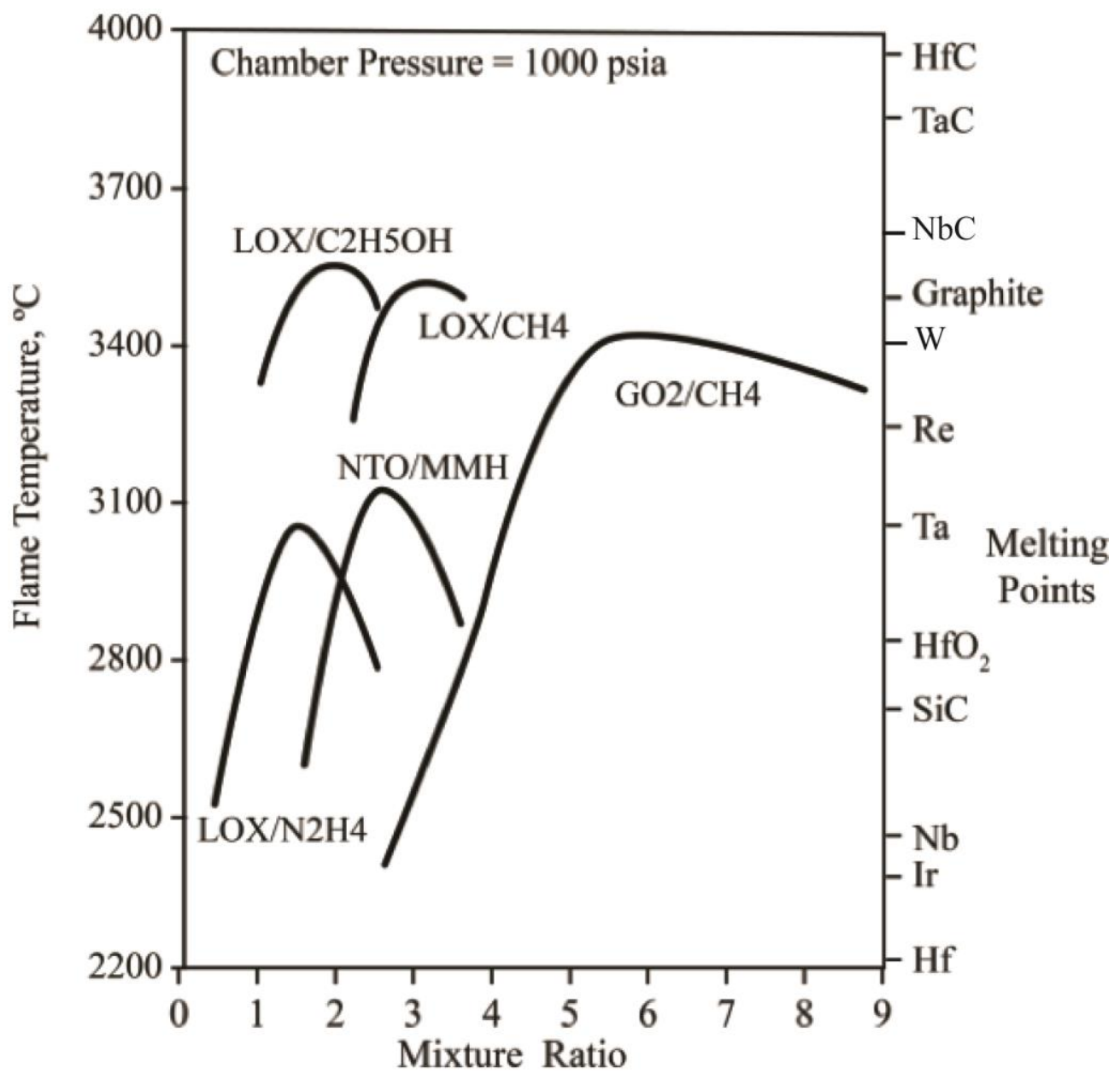

Figure 2: Flame temperatures of various rocket fuels along with the melting points of various high temperature materials [6].

Group IV and V carbides such as Tantalum, Niobium, and Hafnium carbides have melting temperatures in excess of $3600^{\circ} \mathrm{C}$ (Figure 3), making them ideal candidates for high temperature applications [7]. 


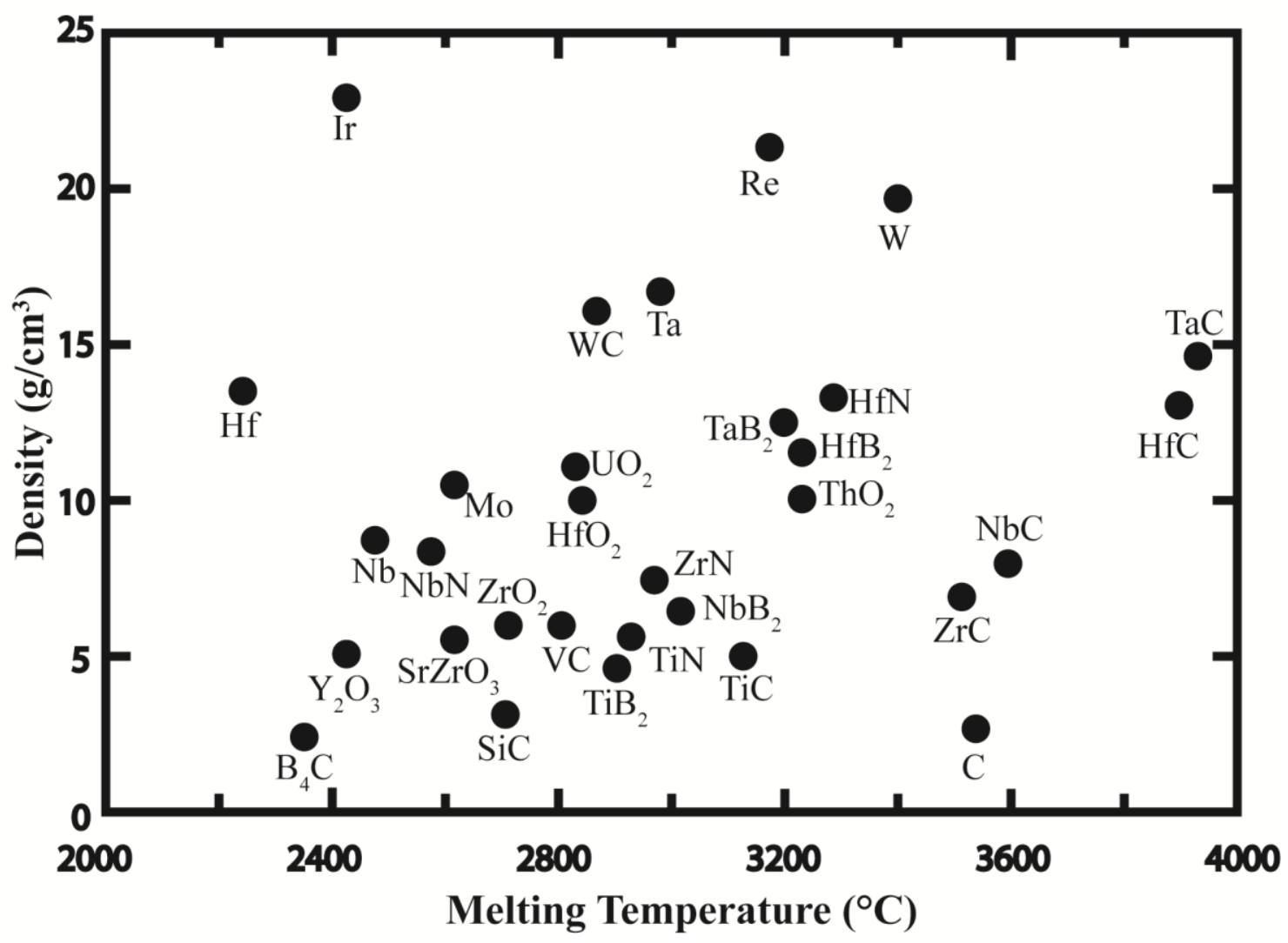

Figure 3: Melting point versus density of select UHTC materials [6].

In order for materials to be used as leading edges or thermal protection systems, they must have high fracture toughness, high mechanical strength, high thermal conductivity, and good oxidation resistance in the extreme environmental conditions. UHTCs are characterized as having good wear resistance, relatively high thermal conductivities, and good mechanical strength at high temperatures.

A major challenge associated with UHTCs is poor consolidation and grain growth during consolidation. UHTC material consolidation is difficult because of their strong covalent bonds, low self-diffusion coefficients, and high melting temperatures. This poses a problem in terms of the reliability of a material being used in such extreme conditions because the microstructure of the material must be as uniform as possible. To 
achieve uniformity, full relative density devoid of any areas of agglomeration and porosity (Figure 4) is necessary.

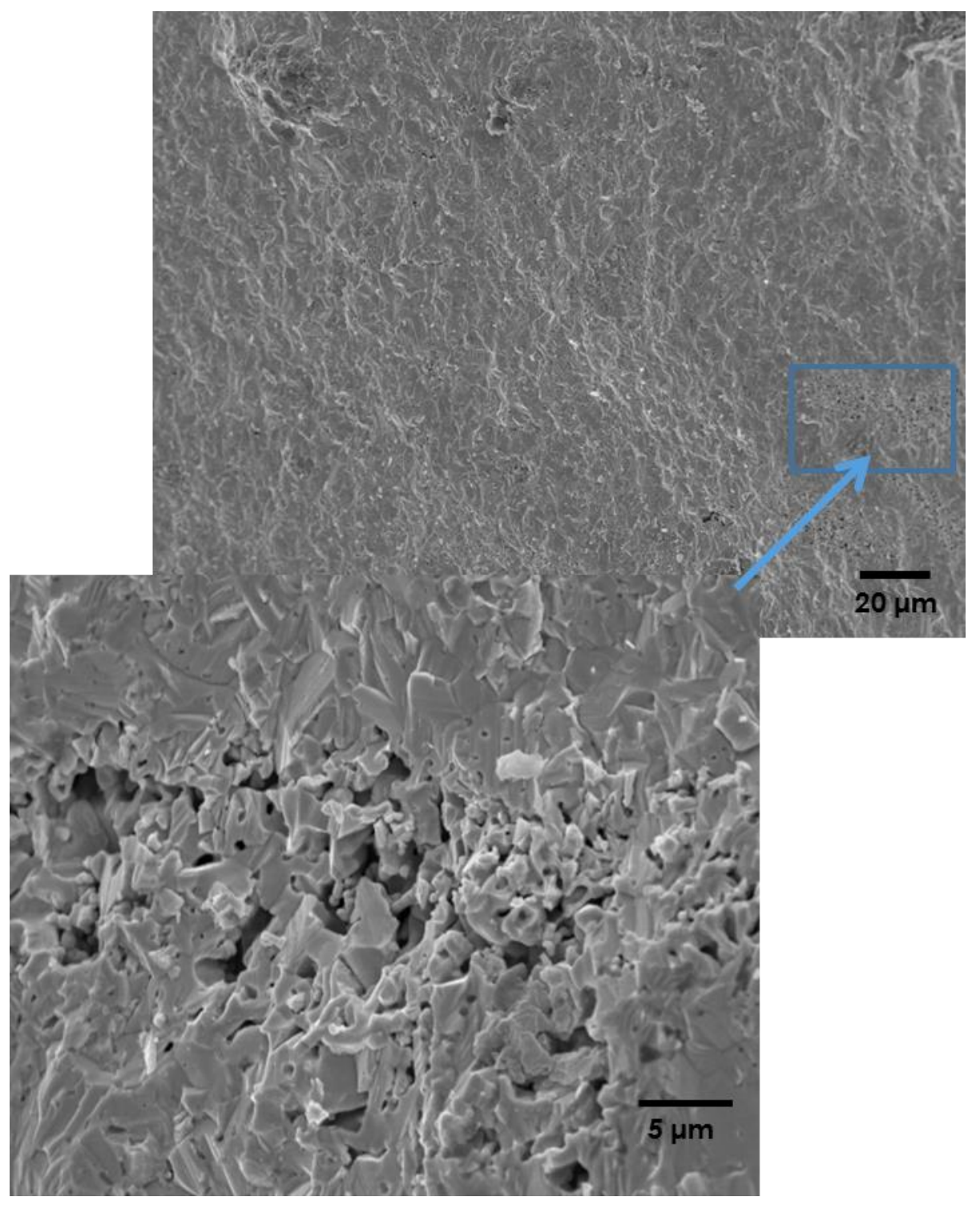

Figure 4: Fracture surface of a spark plasma sintered sample with a magnified view showing a porous region.

The properties of UHTCs are highly influenced by the final grain size and morphology. According to the Hall-Petch relationship, the strength of a material increases as the inverse square root of the grain size which is influenced by the starting powder size, 
added sintering aids, and processing time and temperature [8]. The use of sintering aids can lead to the reduction in temperature when densification begins to take place. Not only will this lead to increased final densification, but it can also reduce the amount of grain growth occurrence.

The leading limitation in using UHTCs in these high temperature applications is their low fracture toughness and poor thermal shock resistance. Even if the fracture toughness is increased, the oxidation resistance of UHTCs needs improvement so the question arises of how this can be accomplished. A possible solution to overcome these limitations is to use nanostructured materials to reinforce the ceramic matrix composite. By reinforcing UHTC composites with nanomaterials such as graphene, the damage tolerance will be increased by enhancing the flexural strength and fracture toughness. Experimentation with the use of nanostructured materials as sintering additives and nanoreinforcement will lead us to a better understanding of the full potential of using UHTC composites in extreme environmental conditions.

One of the hindrances with nanostructure reinforced UHTC composites is the difficulty in observing damage mechanisms and influence on bulk properties at the microscopic level. For this reason, in-situ mechanical property testing techniques will be developed. The ability to observe microscopic deformation in real time can rapidly advance the materials development process. Another reason why advances have been slow is due to the difficulty and expense of testing these materials at the extreme environmental conditions which they will be used in. In order to test the oxidation 
susceptibility in as close to real world operating conditions as possible, plasma flow oxidation testing will be utilized.

\subsection{Graphene Nanoplatelet Reinforcement in Composites}

Graphene is a two-dimensional sheet of $\mathrm{sp}^{2}$ bonded carbon that is known for its extraordinary thermal, mechanical, and electrical properties [9]. The functional properties include a high Young's modulus (0.5 - $1 \mathrm{TPa})$ [10] and a high tensile strength (130 GPa) [11] which is why it is being considered for reinforcement in polymer, metallic, and ceramic composite matrices [12-14]. Graphene Nanoplatelets (GNP) are made up of multiple layers of graphene held together by weak van der Waals forces. GNP are easier and less expensive to form while retaining much of the desired mechanical, thermal, and electrical properties of single layer graphene $[15,16]$. GNP is typically made up of 10-30 sheets of graphene to have a thickness of 3-10 $\mathrm{nm}$ and a width of 1-25 $\mu \mathrm{m}$, providing larger surface areas. Several studies that have used GNP as reinforcement in composites have shown GNP to successfully increase the fracture toughness by absorbing energy that would otherwise result in crack propagation [17-20]. GNP has also been shown to lead to a more refined microstructure by hindering grain growth during sintering [21].

\subsection{Objectives of the Current Research}

The overall objective of this research is to enhance the potential for GNP reinforced UHTC composites to be used in extreme environmental conditions. This research focuses on a pre-alloyed Tantalum-Niobium Carbide system made up of 80 weight percent $\mathrm{TaC}$ and 20 weight percent $\mathrm{NbC}$. Figure 5 shows a graphical representation of the proposed work. 


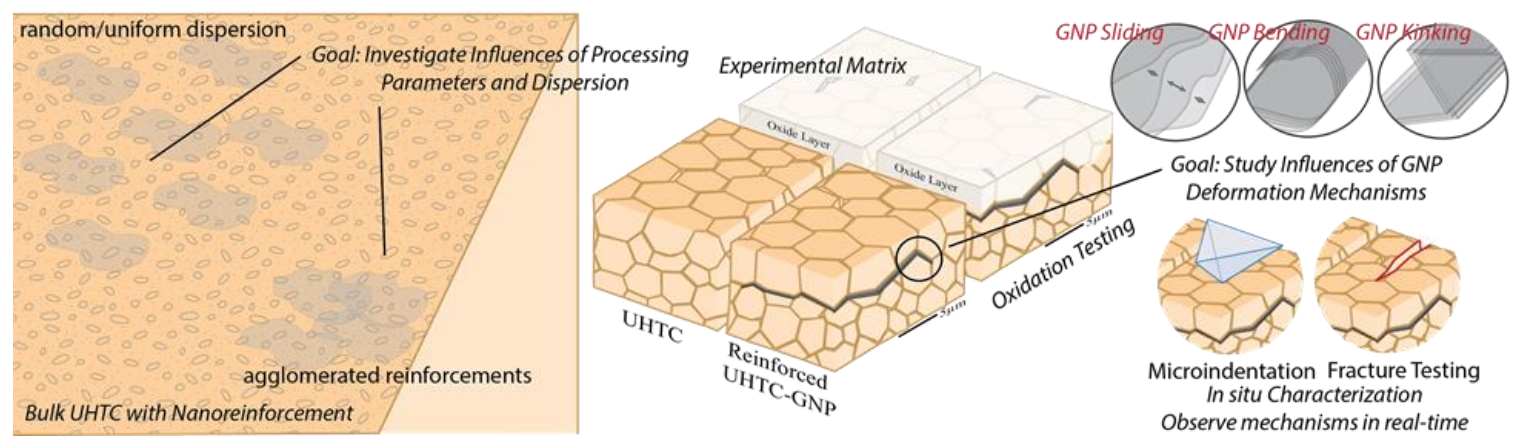

Figure 5: Overview of the proposed work.

The following sections outline the main goals of this work and the specific objectives to meet them.

\subsubsection{Investigate the Toughening Mechanisms of GNP}

The overall toughening benefits of GNP addition to a composite have been previously seen, however they are attributed to various intrinsic mechanisms. In order to increase the toughening benefit that the addition of GNP has to a composite, a fundamental investigation of the GNP mechanisms must first be done. The specific objectives for this are the following:

- Investigate the intrinsic deformation mechanisms in bulk consolidated GNP by in-situ indentation with respect to orientation.

- Analyze the anisotropy of the deformation behavior and the associated energy dissipation capabilities.

\subsubsection{Achieve Full Relative Densification with a Refined Microstructure}

In order to trust the reliability of a material being used in such extreme conditions, the microstructure of the material must be as uniform as possible. To achieve uniformity, full relative density is necessary. The specific objectives for this are the following: 
- Analyze the role of dispersion technique on reduction of agglomeration and uniform constituent dispersion.

- Explore the role that SPS processing parameters (temperature, pressure, dwell time, heating rate) have on microstructure and mechanical properties.

- Explore the use of sintering additives to enhance densification.

- Analyze the formation of secondary phases with the addition of sintering additives.

\subsubsection{Reinforce the Fully Dense Ceramics with GNP for Improved Toughening}

Once full densification of the TaC-NbC compacts can be achieved and the investigation of the intrinsic toughening mechanisms of GNP is complete, fully dense composites will be made with GNP dispersed throughout the matrix for improved toughening. The specific objectives for this are the following:

- Analyze secondary phase formation and retention of GNP characteristics.

- Investigate the addition of GNP on the microstructure of the composite.

- Investigate the fracture toughness with respect to GNP orientation.

- Investigate the bulk fracture strength.

- Analyze the susceptibility to oxidation by plasma flow oxidation testing. 
Chapter II: Literature Review

2.1 Possible UHTC Materials and Why TaC-NbC is Being Investigated

Many different UHTC materials have been investigated for use in extreme environment conditions due to their high melting temperatures in excess of $3000^{\circ} \mathrm{C}$. While the high melting temperature is the property that led to their investigation, their oxidation susceptibility, fracture toughness, thermal conductivity, thermal expansion, and density must also be considered.

Refractory metal diborides, such as $\mathrm{ZrB}_{2}$ and $\mathrm{HfB}_{2}$, have been at the forefront of investigation for UHTCs for extreme environments due to their ability to form refractory oxide scales to withstand temperatures in the 1900 to $2500^{\circ} \mathrm{C}$ range [22]. However, $\mathrm{ZrO}_{2}$ and $\mathrm{HfO}_{2}$ formations become nonstoichiometric by forming oxygen lattice vacancies under low oxygen partial pressure conditions and are readily modified by aliovalent cations of lower valence to form oxygen lattice vacancies allowing rapid oxygen ion transport. Another issue is that at high temperatures, the oxides are tetragonal but upon cooling return to the monoclinic structure with a volume expansion. This can lead to cracking and spalling under thermal transient conditions. One approach to remedy this is to use a cation that can stuff oxygen into the lattice. The best candidates for this are tantalum and niobium which form pentoxides [23].

The ceramic system being explored in this research is a pre-alloyed TantalumNiobium Carbide made up of 80 weight percent TaC and 20 weight percent NbC. Table 1 lists properties of both $\mathrm{TaC}$ and $\mathrm{NbC}$ for comparison. They are both group IV carbides with extremely high melting temperatures $\left(3950^{\circ} \mathrm{C}\right.$ for $\mathrm{TaC}$ and $3600^{\circ} \mathrm{C}$ for $\left.\mathrm{NbC}\right)$. They 
are characterized as having high hardness (16.7 $\mathrm{GPa}$ for $\mathrm{TaC}$ and 19.6 $\mathrm{GPa}$ for $\mathrm{NbC}$ ) [7] and a high elastic modulus (285-560 GPa for $\mathrm{TaC}$ and 338-580 $\mathrm{GPa}$ for $\mathrm{NbC}$ ) [7]. Both $\mathrm{TaC}$ and $\mathrm{NbC}$ are FCC structured materials with very similar lattice constants which form a solid solution from $1500^{\circ} \mathrm{C}$ to melting [24].

Table 1: Property comparison of $\mathrm{TaC}$ and $\mathrm{NbC}$.

\begin{tabular}{|l|c|c|}
\cline { 2 - 3 } \multicolumn{1}{c|}{} & TaC & NbC \\
\hline Molecular Weight & 192.96 & 104.92 \\
\hline Crystal Structure & FCC $(\mathrm{B} 1)$ & FCC $(\mathrm{B} 1)$ \\
\hline Lattice Constants $(\mathrm{nm})$ & 0.4454 & 0.4470 \\
\hline Density $\left(\mathrm{g} / \mathrm{cm}^{3}\right)$ & 14.48 & 7.78 \\
\hline Melting Point $\left({ }^{\circ} \mathrm{C}\right)$ & 3950 & 3600 \\
\hline Vickers Hardness $(\mathrm{GPa})$ & 16.7 & 19.6 \\
\hline Modulus of Elasticity $(\mathrm{GPa})$ & $285-560$ & $338-580$ \\
\hline Transverse Rupture Strength $(\mathrm{MPa})$ & $350-450$ & $300-400$ \\
\hline Coefficient of Thermal Expansion $(1 / \mathrm{K})$ & $6.29 \times 10^{-6}$ & $6.65 \times 10^{-6}$ \\
\hline Thermal Conductivity $(\mathrm{W} / \mathrm{m} * \mathrm{~K})$ & 22 & 14 \\
\hline Electric Resistivity $\left(\mu \Omega^{*} \mathrm{~cm}\right)$ & 25 & 35 \\
\hline
\end{tabular}

While this system may not have as good of an oxidation resistance compared to other UHTC systems, such as $\mathrm{ZrB}_{2}$ and $\mathrm{HfB}_{2}, \mathrm{TaC}$ and $\mathrm{NbC}$ have high resistivity to chemical erosion and show promise for use in non-oxidizing environments such as throat inserts for aluminum burning rockets. Table 2 shows the temperatures at which different compositions begin to react to form oxides after being coated with molten $\mathrm{Al}_{2} \mathrm{O}_{3}$ in solid Aluminum burning rocket nozzles. Due to the reaction temperatures being $>3000^{\circ} \mathrm{C}$ $\left(5432^{\circ} \mathrm{F}\right)$ for the different stoichiometries of $\mathrm{TaC}$ and $\mathrm{NbC}$, these materials are the focus of this research. 
Table 2: Reaction initiation temperatures of compositions after being coated with molten $\mathrm{Al}_{2} \mathrm{O}_{3}$ in solid Aluminum burning rocket nozzles [25].

\begin{tabular}{|c|c|c|}
\hline \multicolumn{3}{|c|}{$\begin{array}{c}\text { Reaction Initiation Temperatures (RIT) of compositions after being } \\
\text { coated with molten } \mathbf{A l}_{2} \mathbf{O}_{3} \text { in Aluminum burning rocket nozzle }\end{array}$} \\
\hline Formula & RIT $\left({ }^{\circ} \mathrm{C}\right)$ @ 1 Atmosphere & Solidus $\left({ }^{\circ} \mathrm{C}\right)$ \\
\hline $\mathrm{TaC}$ & 3006 & 3445 \\
\hline $\mathrm{TaC}_{0.9}$ & 3049 & 3899 \\
\hline $\mathrm{Ta}_{0.9} \mathrm{~W}_{0.1} \mathrm{C}$ & 3051 & 3196 \\
\hline $\mathrm{Ta}_{2} \mathrm{C}$ & 3082 & 3332 \\
\hline $\mathrm{Ta}_{0.36} \mathrm{Nb}_{0.66} \mathrm{C}$ & 3092 & 3199 \\
\hline $\mathrm{TaC}_{0.8}$ & 3097 & 3749 \\
\hline $\mathrm{NbC}$ & 3138 & 3304 \\
\hline $\mathrm{TaC}_{0.7}$ & 3150 & 3510 \\
\hline $\mathrm{Ta}_{0.36} \mathrm{Nb}_{0.65} \mathrm{C}_{0.826}$ & 3188 & 3650 \\
\hline $\mathrm{NbC}_{0.9}$ & 3196 & 3521 \\
\hline $\mathrm{TaC}_{0.6}$ & 3209 & 3610 \\
\hline $\mathrm{NbC}_{0.8}$ & 3253 & 3510 \\
\hline $\mathrm{NbC}_{0.7}$ & 3311 & \\
\hline & & \\
\hline
\end{tabular}

While there has been extensive study on the $\mathrm{TaC}$ system, there has yet to be any study on a $\mathrm{TaC}-\mathrm{NbC}$ system for an extreme environment material. One of the biggest advantages of $\mathrm{NbC}$ addition is that the density is almost half that of $\mathrm{TaC}\left(\mathrm{NbC}=7.79 \mathrm{~g} / \mathrm{cm}^{3}, \mathrm{TaC}=\right.$ $14.50 \mathrm{~g} / \mathrm{cm}^{3}$ ) [7]. Because this system is being considered for high temperature aerospace applications and rocket nozzle throat inserts, the reduction in weight due to a decrease in the density will make this system more viable and cost effective.

\subsection{Consolidation Methods for TaC based UHTCs}

Tantalum Carbide, as well as other UHTC materials such as NbC, VC, and HfC, has been consolidated into bulk form using various processing approaches (outlined in Table 3) including hot pressing [26-31], pressureless sintering [32, 33], vacuum plasma 
spraying [34], high frequency induction heating [35, 36], and spark plasma sintering [37-

40]. Differences in heating and cooling rates, temperatures, dwell times, and pressures

will impact the phase formation, microstructures, and densification of the final product.

Table 3: Comparison of consolidation methods for TaC based UHTCs.

\begin{tabular}{|l|l|l|}
\hline Consolidation Method & Mechanisms of Consolidation & \multicolumn{1}{|c|}{ Remarks } \\
\hline Hot Pressing & $\begin{array}{l}\text { Uniaxial pressure and inductive } \\
\text { or convective heating }\end{array}$ & $\begin{array}{l}\text { Slow heating and long } \\
\text { dwell times result in large } \\
\text { grain growth }\end{array}$ \\
\hline Pressureless Sintering & $\begin{array}{l}\text { High temperature sintering } \\
\text { without pressure after being } \\
\text { cold pressed at high pressures }\end{array}$ & $\begin{array}{l}\text { Can avoid density } \\
\text { variations in the sintered } \\
\text { compact normally caused } \\
\text { by added pressure. High } \\
\text { temperatures can lead to } \\
\text { grain growth }\end{array}$ \\
\hline $\begin{array}{l}\text { Vacuum Plasma } \\
\text { Spraying }\end{array}$ & $\begin{array}{l}\text { Powder injected into a plasma } \\
\text { plume before being deposited } \\
\text { on a substrate }\end{array}$ & $\begin{array}{l}\text { Can only be used to make } \\
\text { thin coatings however } \\
\text { can be used for making } \\
\text { difficult shapes }\end{array}$ \\
\hline Induction Heating & $\begin{array}{l}\text { Heating is thru high frequency } \\
\text { electricity around an } \\
\text { electrically conductive material } \\
\text { or die with or without pressure }\end{array}$ & $\begin{array}{l}\text { Fast heating that works } \\
\text { best for electrically } \\
\text { conductive powders, } \\
\text { otherwise it is conductive } \\
\text { heating through a die }\end{array}$ \\
\hline Spark Plasma Sintering & $\begin{array}{l}\text { Electric DC current flowing } \\
\text { through a die (usually graphite) } \\
\text { creating high temperatures } \\
\text { through an electric field. Uses } \\
\text { uniaxial pressure for particle } \\
\text { rearrangement and enhanced } \\
\text { sintering }\end{array}$ & $\begin{array}{l}\text { The fast heating rate and } \\
\text { direct current heating } \\
\text { leads to densification at } \\
\text { lower temperatures and } \\
\text { shorter dwell times } \\
\text { leading to a more refined } \\
\text { microstructure }\end{array}$ \\
\hline
\end{tabular}

In the following subsections, each of the processing methods will be outlined and the characteristics of the resulting bulk consolidated material will be compared.

\subsubsection{Hot Pressing}

Hot pressing is one of the most widely used processes to consolidate UHTC materials. It involves the slow application of heat and uniaxial pressure to allow 
significant plastic flow of the material to increase relative densification. Zhang et al. [29, 30] achieved $94 \%$ relative densification of $\mathrm{TaC}$ by hot pressing at $2300^{\circ} \mathrm{C}$ for 45 minutes with a pressure of $30 \mathrm{MPa}$. When the temperature was increased to $2400^{\circ} \mathrm{C}$, the relative densification increased to $96 \%$ but saw an increase in average grain size from $1.6 \mu \mathrm{m}$ to $2.8 \mu \mathrm{m}$ (Figure 6).
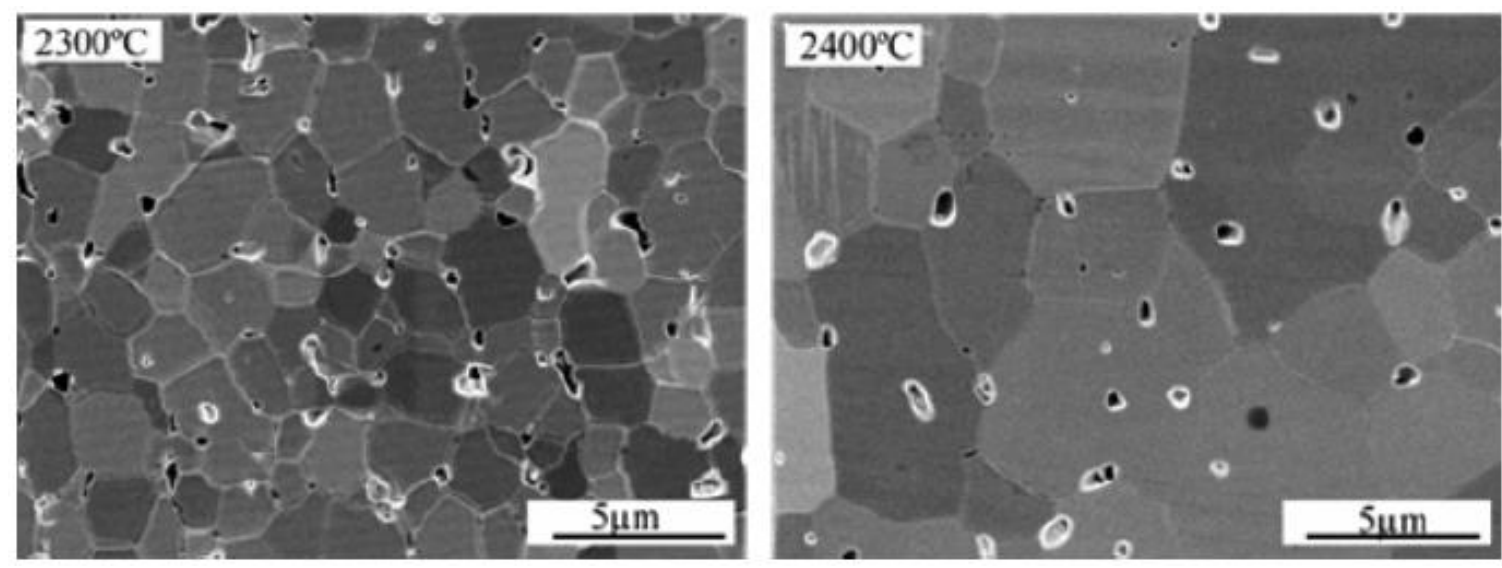

Figure 6: Microstructures of tantalum carbide hot pressed without additives at (a) $2300^{\circ} \mathrm{C}$ and (b) $2400^{\circ} \mathrm{C}$ [29].

Hackett et al. [26] hot pressed $\mathrm{TaC}$ with an average particle size of $1.04 \mu \mathrm{m}$ at $1800^{\circ} \mathrm{C}$ for 2 hours at a pressure of $28 \mathrm{MPa}$ using heating rates of $10-15^{\circ} \mathrm{C} /$ minute. They were able to reach $97 \%$ relative densification but the grain growth was immense with a final average grain size of $7.7 \mu \mathrm{m}$. While hot pressing achieves high densification which can be further enhanced by the use of sintering additives, the process entails slow heating rates, high temperatures, and long dwell times that lead to enhanced grain growth.

\subsubsection{Pressureless Sintering}

The process of pressureless sintering involves high temperatures without any applied pressure; however the samples are normally pressed at high pressures beforehand. 
Liu et al. [32] prepared monolithic $\mathrm{TaC}$ samples by first cold isostatically pressing at 250 MPa followed by pressureless sintering between $2200-2400^{\circ} \mathrm{C}$ using different sized starting powder $(2 \mu \mathrm{m}$ and $0.6 \mu \mathrm{m})$. SEM fractographs (Figure 7) of each starting powder size $(\mathrm{SB}=2 \mu \mathrm{m}, \mathrm{SC}=0.6 \mu \mathrm{m})$ sintered at 2200,2300 , and $2400^{\circ} \mathrm{C}$ show that grain growth occurs with increasing temperature from $6 \mu \mathrm{m}$ to $8 \mu \mathrm{m}$ to $12 \mu \mathrm{m}$ for the $2 \mu \mathrm{m}$ starting powder and from $1.5 \mu \mathrm{m}$ to $2 \mu \mathrm{m}$ to $4 \mu \mathrm{m}$ for the $0.6 \mu \mathrm{m}$ starting powder. A maximum density of $97.5 \%$ was achieved at $2400^{\circ} \mathrm{C}$. 

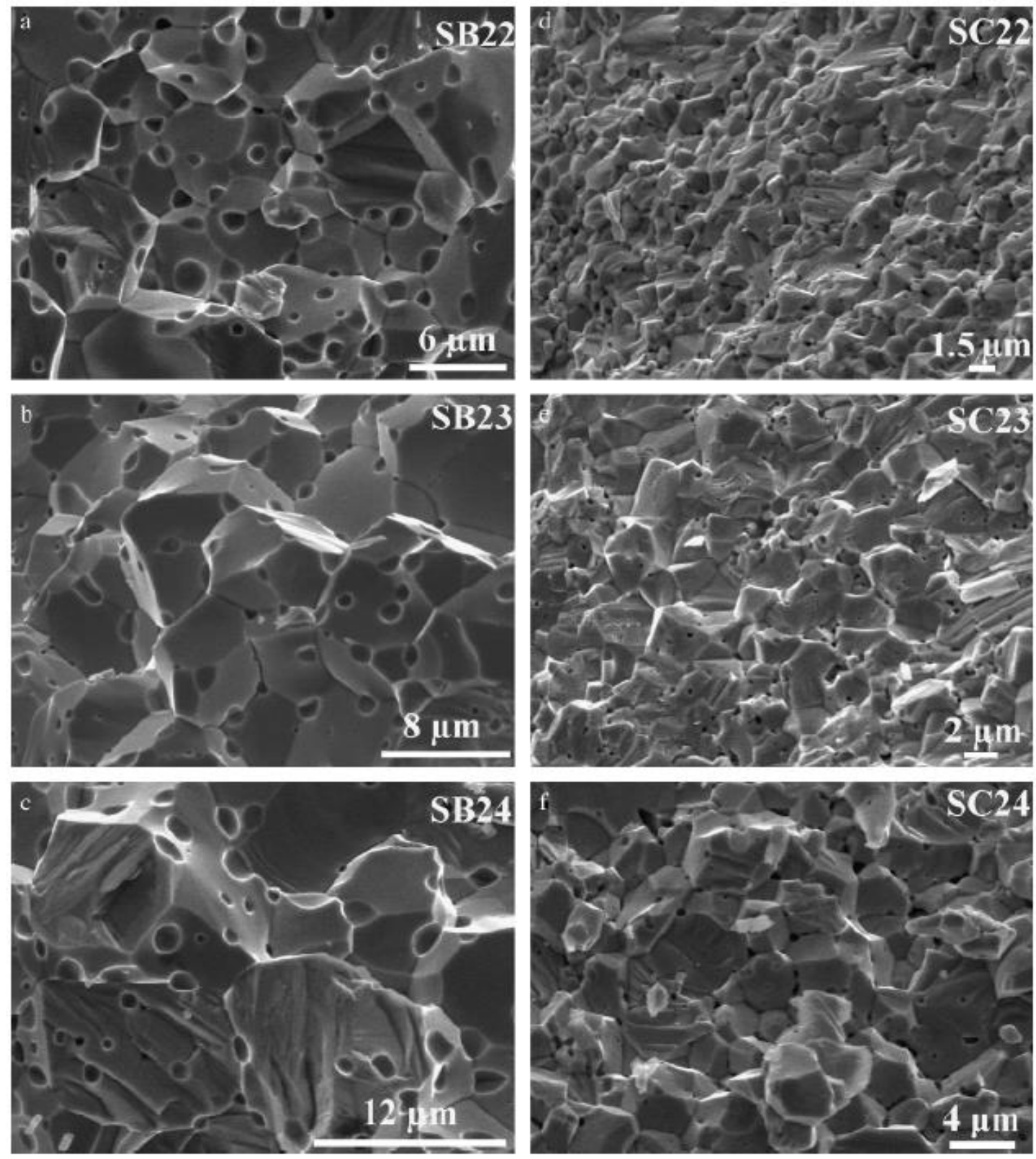

Figure 7: Scanning electron micrographs of the morphologies of the fracture surfaces of the tantalum carbide ceramics. (a) SB22, (b) SB23, (c) SB24, (d) SC22, (e) SC23, and (f) $\mathrm{SC} 24$ [32].

\subsubsection{Vacuum Plasma Spraying}

Vacuum plasma spraying (VPS) allows for the fabrication of thin walled structures as well as contoured structures. The VPS process entails feeding the powder 
into a plasma plume (temperatures in excess of $10,000^{\circ} \mathrm{C}$ ) to partially melt the material which is then deposited on a substrate creating a mechanically bonded layered structure. Belani et al. [34] fabricated freestanding $\mathrm{TaC}$ structures by deposition on a graphite substrate (Figure 8).

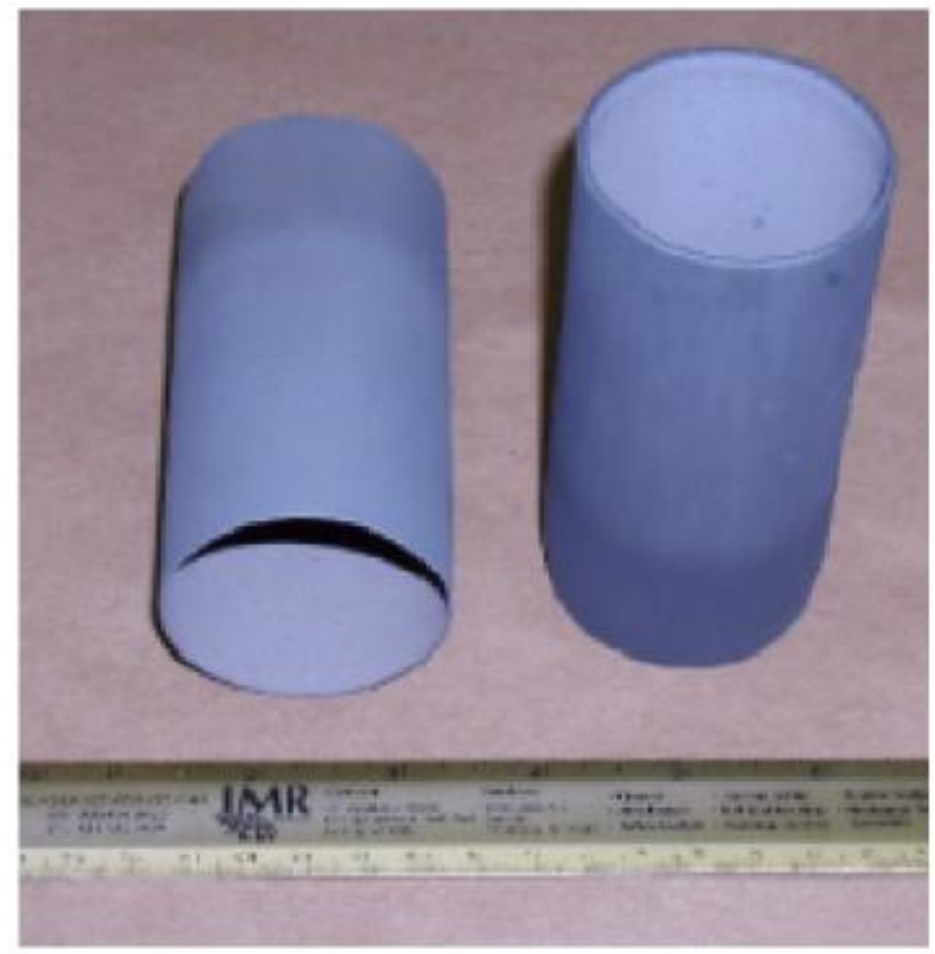

Figure 8: Vacuum plasma-sprayed TaC cylinder $50 \mathrm{~mm}$ diameter, $100 \mathrm{~mm}$ height, and $1.5 \mathrm{~mm}$ thick [34].

The resulting microstructure displayed anisotropic behavior attributed to incomplete formation of grain boundaries, incomplete transformation of the phases, non-uniform distribution of carbon fraction, and polygonization of the grains. This led to varied hardness values in the axial direction $(21.25 \pm 2.69 \mathrm{GPa})$ compared to the transverse direction $(26.90 \pm 9.42 \mathrm{GPa})$. 


\subsubsection{High Frequency Induction Heating}

High frequency induction heating is a sintering process that allows for rapid consolidation of electrically conductive materials. The rapid heating and high temperatures are generated by passing alternating current through a coil which is around the material to be sintered. Kim et al. $[35,36]$ used high frequency induction heating to sinter both $\mathrm{TaC}$ and $\mathrm{NbC}$. The powders were placed in a graphite die and put under an applied pressure of $80 \mathrm{MPa}$ before being sintered for 2-3 minutes. The high frequency induction heating was controlled by output control which reached $80 \%$ of the total $15 \mathrm{~kW}$ power for $\mathrm{TaC}$ and $70 \%$ for $\mathrm{NbC}$. These values correspond to starting temperatures between $1050-1425^{\circ} \mathrm{C}$. For $\mathrm{TaC}$, final relative density varied from $68 \%$ to $96 \%$ depending on the starting powder sizes of between $935 \mathrm{~nm}$ and $33 \mathrm{~nm}$, respectively. For $\mathrm{NbC}$, final relative density varied from $73 \%$ to $98 \%$ depending on the starting powder sizes between $3.7 \mu \mathrm{m}$ and $1.8 \mu \mathrm{m}$, respectively.

\subsubsection{Spark Plasma Sintering}

Spark plasma sintering will be discussed in detail in the following section (section 2.3).

\subsection{Spark Plasma Sintering}

Spark Plasma Sintering, also known as Field Activated Sintering Technique (FAST) or Pulsed Electric Current Sintering (PECS), utilizes a uniaxial force and a direct electric current to perform high speed consolidation of powders. Compared to other consolidation techniques, such as hot pressing, which heat the sample by conduction from the external surface of the container to the powder, the heating of an SPS sample by 
passing direct current through the die and powder is much faster [41]. The high heating rate enhances densification while minimizing grain growth that occurs when holding at a high temperature for an extended period. Compared with the other consolidation techniques mentioned in section 2.2 that could only reach a densification of $97.5 \%$ for the case of TaC consolidation, spark plasma sintering has achieved full densification at lower temperatures and shorter dwell times [37].

The SPS system (Figure 9) consists of a graphite sintering die which is loaded with powder, upper and lower graphite punches, and graphite sintering spacers stacked between the upper and lower uniaxial pressure rams that are also attached to the electrodes allowing high DC current to flow through. The process is carried out under vacuum atmosphere $\left(10^{-2}\right.$ to $10^{-4}$ bar) and temperature is measured using a pyrometer.

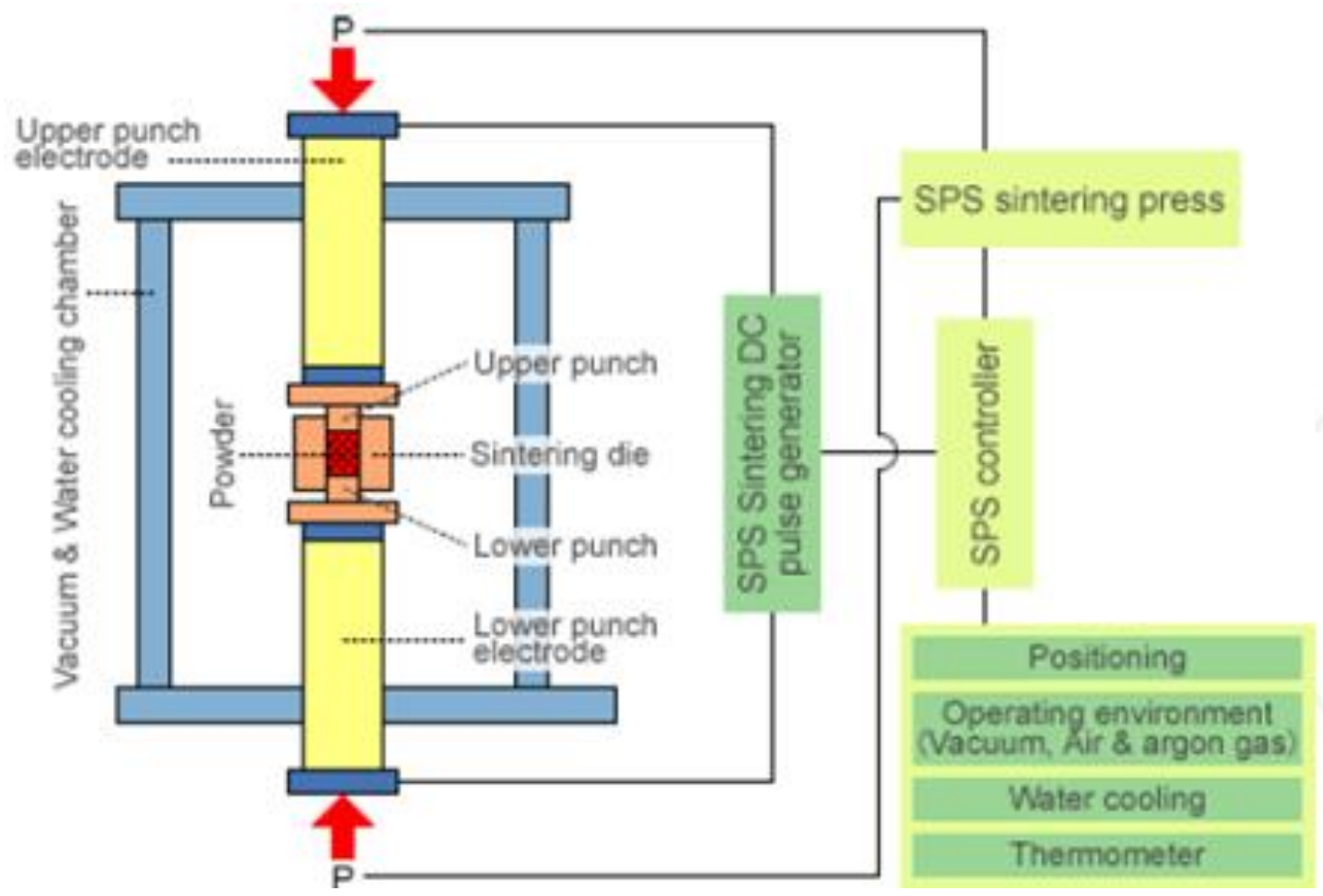

Figure 9: Schematic of the Spark Plasma Sintering System. 
Joule heating, due to the passage of high electric current through the particles, assists in the diffusion between powder particles. Therefore, materials with higher electrical conductivity will have a more uniform microstructure due to a more uniform distribution of heating. The application of pressure during the SPS process has a direct effect on particle re-arrangement which leads to increased surface area allowing better diffusion and greater densification. The ability to use SPS in the formation of UHTCs shows promise to achieve fully dense composites, with limited grain growth, and with limited microstructural defects and enhanced mechanical properties. In the following section, the different SPS processing parameters will be discussed.

\subsubsection{SPS Processing Parameters}

The spark plasma sintering process can be refined by configuring various processing parameters. These parameters include thermal effects such as maximum temperature, heating rate, and dwell time as well as mechanical effects from applied pressure. These parameters will be discussed in the following subsections.

\subsubsection{Temperature}

The temperature at which sintering takes place with SPS processing can be much lower when compared to other consolidation techniques. This reduction in densification temperature results in a reduction of grain growth leading to a more refined microstructure. Liu et al. [40] used SPS to sinter $\mathrm{TaC}$ at an equivalent temperature of $1400^{\circ} \mathrm{C}$ and a dwell time of approximately 5 minutes by using a heating schedule rearrangement consisting of periods of cooling before heating again. They concluded that the low temperature sintering prevented the non-densifying evaporation-condensation and 
exploited the mass diffusion kinetics in the range of $1213-1446^{\circ} \mathrm{C}$. They were able to achieve a relative density greater than $96 \%$ which compared to a hot pressed sample consolidated at $2100^{\circ} \mathrm{C}$ for 2 hours.

\subsubsection{Heating Rate}

The high heating rates is one of the main advantages that SPS has over conventional sintering techniques such as hot pressing. With SPS, the heating rate can be as high as $1000^{\circ} \mathrm{C} / \mathrm{min}$. whereas the typical heating rate for hot pressing is less than $50^{\circ} \mathrm{C} /$ minute. Rapidly heating the sample leads to a greater densification rate while limiting the time for coarsening mechanisms like surface diffusion to take place. This results in a more refined microstructure when compared to other consolidation techniques.

\subsubsection{Dwell Time}

The dwell time at the holding temperature has to be chosen so as to achieve maximum densification while limiting grain growth. One of the output parameters when processing by SPS is displacement of the uniaxial pressure rams. An analysis of the ram displacement can help to determine the appropriate dwell time for a specific process schedule. This will be discussed in detail in section 3.3.

\subsubsection{Pressure}

The uniaxial force that generates the mechanical pressure during sintering leads to improved contact between powder particles, which leads to enhancement of the existing densification mechanisms such as grain boundary diffusion, lattice diffusion, and viscous flow as well as possibly activating new densification mechanisms such as plastic 
deformation or grain boundary sliding [42]. The addition of pressure is not expected to enhance the non-densifying mechanisms such as surface diffusion or evaporation/condensation [43]. Bakshi et al. [37] consolidated TaC by SPS at a temperature of $1850^{\circ} \mathrm{C}$ and varying pressures of 100,255 , and $363 \mathrm{MPa}$. At a pressure of $100 \mathrm{MPa}$, a relative density of $89 \%$ was achieved, whereas full densification was achieved for pressures of $255 \mathrm{MPa}$ and up (Figure 10).

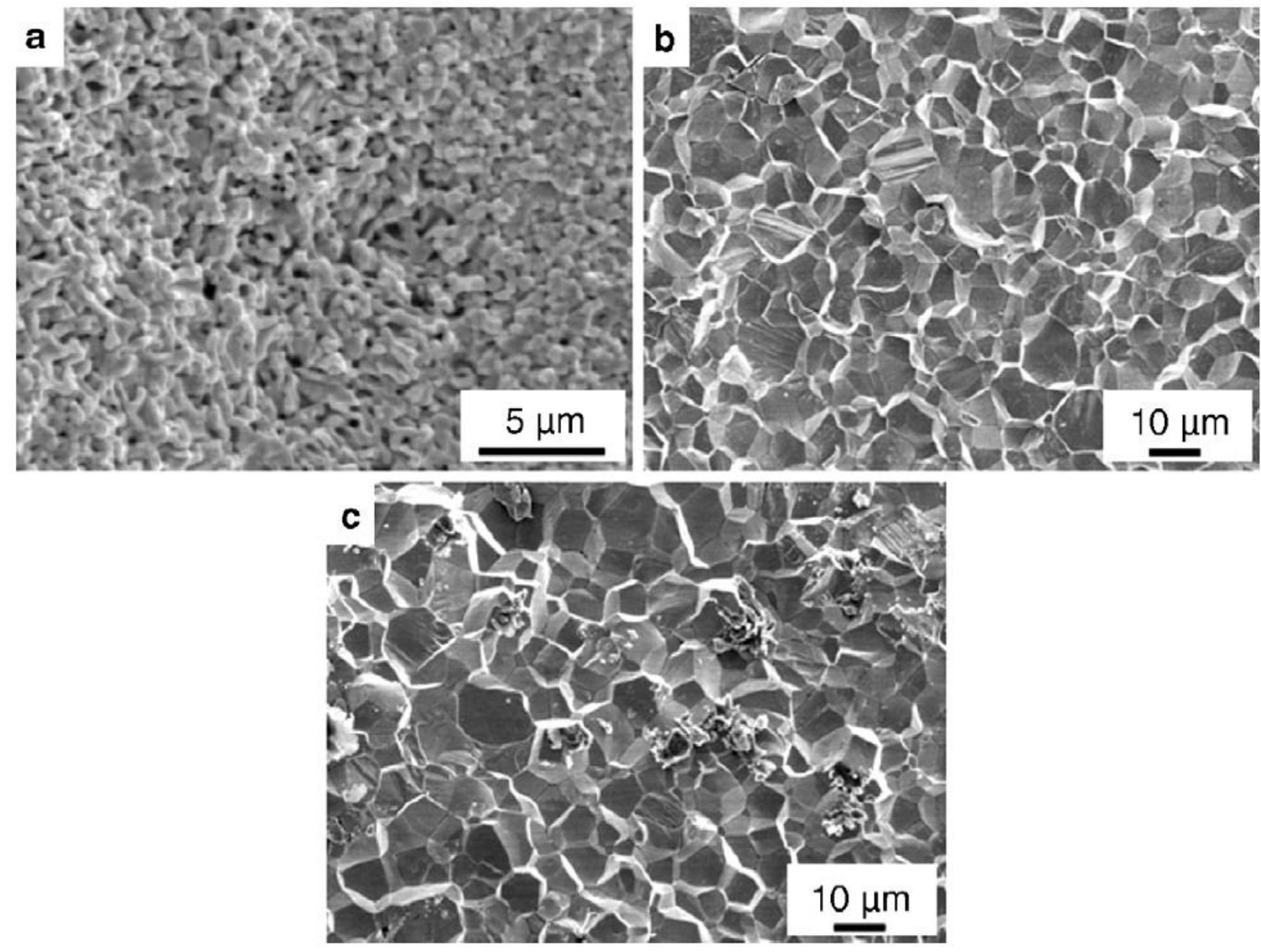

Figure 10: SEM micrographs of fracture surfaces of TaC samples prepared by SPS at (a) $1850^{\circ} \mathrm{C}$ and $100 \mathrm{MPa}$, (b) $1850^{\circ} \mathrm{C}$ and $255 \mathrm{MPa}$, and (c) $1850^{\circ} \mathrm{C}$ and $363 \mathrm{MPa}$ [37].

2.4 Use of Sintering Additives

Much of the research into the densification of ceramics is focused on processing parameters and various consolidation techniques. Another way to increase the 
densification of ceramics is by using sintering additives that can lead to a reduction in temperature when sintering takes place. In addition to the increase in densification, the lower temperatures can have a positive effect on the final microstructure by reducing the grain growth occurrence. When considering possible sintering additives, it is very important to consider both their properties and the properties of possible secondary phases at the extreme temperatures that these UHTCs will be used in. In this section, various groups of sintering additives will be discussed in terms of their ability to enhance densification and microstructure of UHTCs.

One group of sintering aids that has been explored is metallic additives. Due to the sintering temperatures being higher than the melting points of the metallic additives, the additives melt and wet the UHTC particles. This wetting has the potential to reduce surface diffusion that can facilitate grain coarsening. Liu et al. [44] used SPS to sinter $\mathrm{TaC}$ with the addition of $5 \mathrm{~mol} . \% \mathrm{Al}, \mathrm{Cu}, \mathrm{Ag}$, and $\mathrm{Au}$ (Figure 11). Full density while limiting grain growth and an increase in flexural strength were seen with the addition of Au when sintering at $1700^{\circ} \mathrm{C}$ for 5 minutes under $30 \mathrm{MPa}$ pressure. 

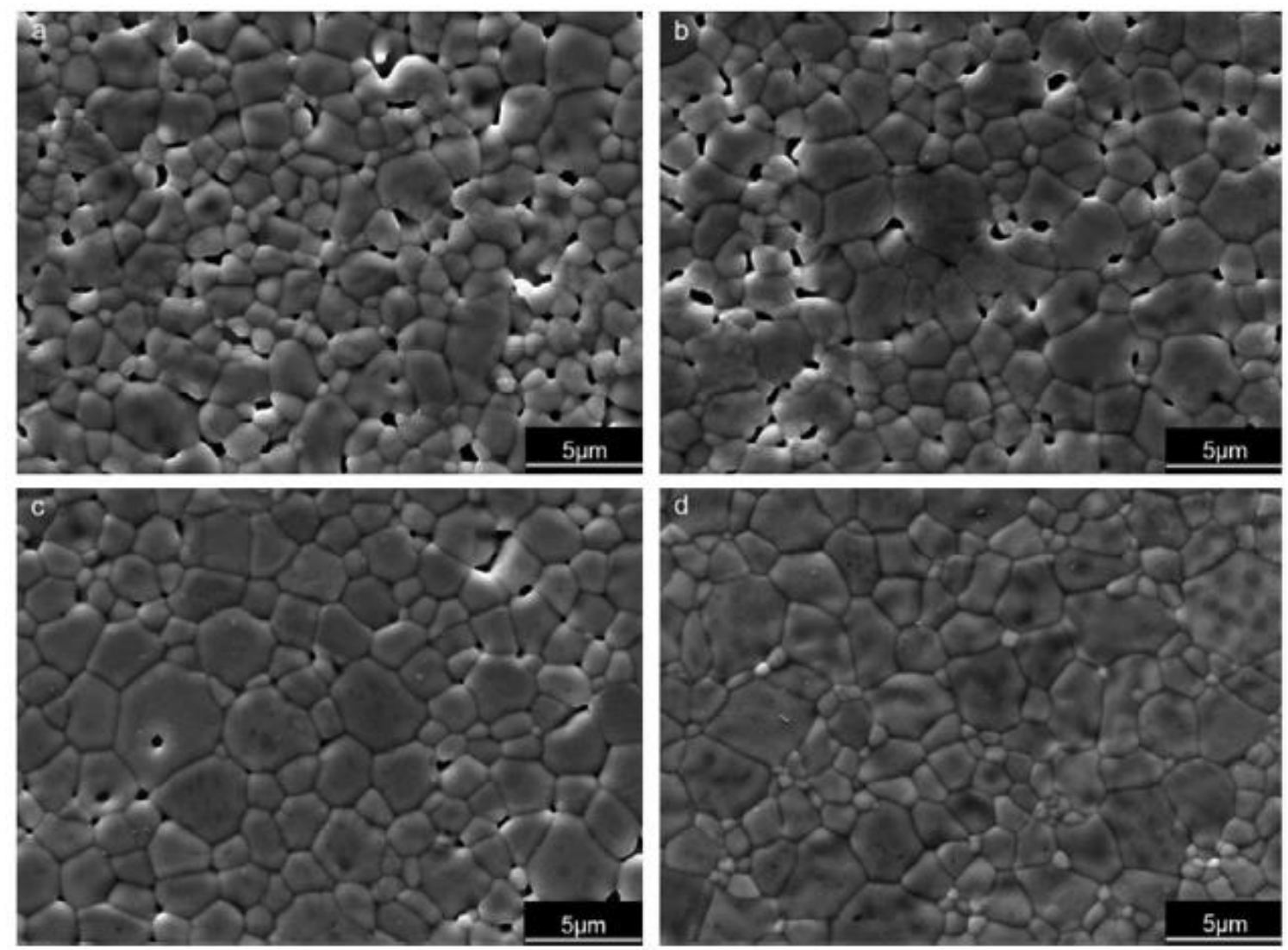

Figure 11: Secondary electron images of the microstructures of the consolidated $\mathrm{TaC}$ materials with (a) $5 \mathrm{~mol} . \% \mathrm{Al}$, (b) $5 \mathrm{~mol} \% \mathrm{Cu}$, (c) $5 \mathrm{~mol} \% \mathrm{Ag}$, and (d) $5 \mathrm{~mol} . \% \mathrm{Au}$ additions [44].

While these additives enhanced the densification of the ceramic composite, the composites failed when heated to just $1200-1600^{\circ} \mathrm{C}$ due to their extreme susceptibility to oxidation. Furthermore, the presence of the liquid phase at the grain boundaries formed by metallic additives limits the usefulness of the UHTCs for the high temperature applications [31].

Other sintering additives that have previously been shown to enhance densification while better maintaining the high temperature properties of the ceramic include carbon, $\mathrm{TaB}_{2}, \mathrm{TaSi}_{2}, \mathrm{MoSi}_{2}, \mathrm{SiC}, \mathrm{B}$ C, and $\mathrm{Si}[28,30,31,33,37,39]$. Densification of a non-oxide ceramic, such as $\mathrm{TaC}$, has been enhanced by adding free 
carbon or $\mathrm{B}_{4} \mathrm{C}$ which act as reducing agents that react with sintering inhibiting oxide impurities, such as $\mathrm{Ta}_{2} \mathrm{O}_{5}$, on the particle surfaces [30]. Sintering additives can also enhance densification by physically pinning grains to mitigate grain growth and reduce the possibility of trapped porosity. Zhang et al. [28] added $10 \mathrm{wt} . \% \mathrm{TaB}_{2}$ to $\mathrm{TaC}$ and hot pressed at $2100^{\circ} \mathrm{C}$. With the addition of $\mathrm{TaB}_{2}$, the relative densification increased from $85 \%$ to $98.6 \%$ which they attributed to grain pinning. The mechanical properties of these UHTC composites with sintering additive are usually as good as or better than the monolithic. Liu et al. [39] prepared $\mathrm{TaC}$ with 20 vol.\% $\mathrm{SiC}$ as sintering additive and saw an increase in Young's modulus, Vickers hardness, flexural strength, and fracture toughness. The increase in mechanical properties can be attributed to the reduction of residual porosity and/or the properties of the secondary phases formed.

\subsection{Materials for Reinforcement in UHTC Composites}

Recently, there has been increasing investigation into the addition of materials for reinforcement to UHTCs due to the fact that their low fracture toughness is a main drawback for their use in extreme environment applications. The main reinforcements that have been used for UHTCs and TaC-based UHTCs in particular are carbon forms and other ceramics. The first carbon form used for UHTC reinforcement was carbon fibers. Gibson et al. [45] reinforced TaC with carbon fibers in the late 1970s but found them difficult to disperse without damaging their structure. A cumbersome multi-step process was used to ensure the carbon fibers were not damaged. The discovery of the next generation nano-carbon forms, such as carbon nanotubes and more recently graphene has since been the focus of reinforcement for UHTCs. In the following sections, 
the various reinforcement materials will be discussed in terms of properties, strengthening mechanisms, ease of dispersion, and survival of structure.

\subsubsection{Ceramic Reinforcement to UHTC Composites}

Various ceramic particles, mainly $\mathrm{B}_{4} \mathrm{C}$ and $\mathrm{SiC}$, have been explored as reinforcement materials for TaC-based UHTCs. The ceramic additives have been shown to improve the density, inhibit grain growth, and in some cases increase the mechanical properties (hardness, elastic modulus, and fracture toughness) of TaC composites. While the mechanical properties of the composite may increase compared to the monolithic, the increase in the mechanical properties is most often attributed to the sintering enhancement leading to increased densification and refined grain size. Zhang et al. [29] found that hot pressing $\mathrm{TaC}$ with 1 and $2 \mathrm{wt} \% \mathrm{~B}_{4} \mathrm{C}$ addition at $2100^{\circ} \mathrm{C}$ led to an increase in Vickers hardness of $7.1 \%$ and $15.6 \%$, respectively. The increase in hardness is attributed to the increased densification (from $94 \%$ for pure $\mathrm{TaC}$ to $>98 \%$ with the addition of $\mathrm{B}_{4} \mathrm{C}$ ) as well as the formation of the secondary phase $\mathrm{TaB}_{2}$ that has a reported hardness of $24.5 \mathrm{GPa}$. The elastic modulus decreased slightly (from $472 \mathrm{GPa}$ to $470 \mathrm{GPa}$ ) compared to the baseline $\mathrm{TaC}$ sample which was attributed to excess carbon after the reaction between $\mathrm{TaC}$ and $\mathrm{B}_{4} \mathrm{C}$ that formed the $\mathrm{TaB}_{2}$. The fracture toughness also decreased with the addition of $\mathrm{B}_{4} \mathrm{C}$ and was attributed to the lack of toughening mechanisms operating in the system.

The processing of composites has also been shown to affect the toughening increase due to the addition of reinforcement. Bakshi et al. [37] consolidated TaC with 1 wt. $\% \mathrm{~B}_{4} \mathrm{C}$ addition by SPS at $1850^{\circ} \mathrm{C}$ and pressures of 100,255 , and $363 \mathrm{MPa}$. Again, the 
addition of the $\mathrm{B}_{4} \mathrm{C}$ acted as a sintering additive, increasing the densification from $89 \%$ to $97 \%$ for the samples consolidated at $100 \mathrm{MPa}$. They observed that the reaction between the $\mathrm{TaC}$ and $\mathrm{B}_{4} \mathrm{C}$ led to the formation of $\mathrm{TaB}_{2}$ and excess carbon at the grain boundaries (Figure 12). This formation led to the pinning of the $\mathrm{TaC}$ grains resulting in a reduction in grain growth.
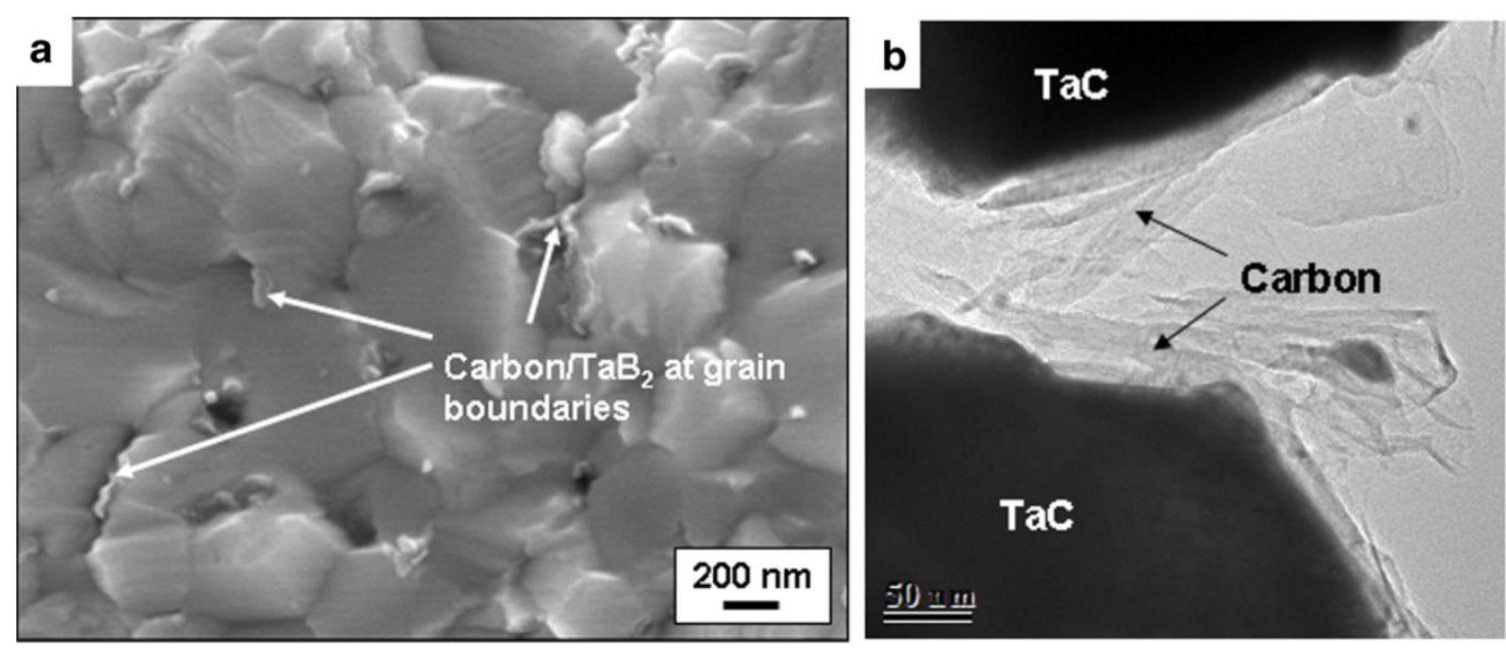

Figure 12: (a) SEM micrograph of the fracture surface of TaC-BC-363 sample indicating the presence of Carbon/ $\mathrm{TaB}_{2}$ at $\mathrm{TaC}$ grain boundaries, (b) TEM image of TaC-BC-363 sample showing the presence of carbon flakes between TaC grains [37].

A 93\% improvement in relative fracture toughness was reported in the sample consolidated at $363 \mathrm{MPa}$. The increase in toughness was attributed to the localized plastic deformation that occurred when processing at such a high pressure that could dissipate energy. The increase in toughness could also be due to the presence of the carbon and $\mathrm{TaB}_{2}$ at the grain boundaries which can increase the fracture energy.

The addition of $\mathrm{SiC}$ to UHTC composites has the same sintering aid effect as $\mathrm{B}_{4} \mathrm{C}$ addition but also shows additional possible toughening mechanisms. SiC has been the most attractive ceramic reinforcement additive to UHTCs due to its high hardness (24.5- 
28.2 GPa), flexural strength (440-500 MPa), elastic modulus (440-475 GPa), thermal conductivity (40-41 W/m* $\left.{ }^{*} \mathrm{C}\right)$, and oxidation resistance. Liu et al. [39] sintered TaC with $20 \mathrm{vol} . \% \mathrm{SiC}$ addition by SPS at $1900^{\circ} \mathrm{C}$ for 5 minutes. They reported an increase in flexural strength of $40 \%$ over the monolithic TaC sample (from 500 to $715 \mathrm{MPa}$ ) as well as a $97 \%$ increase in fracture toughness with the addition of the $\mathrm{SiC}$ (from 3.4 to 6.7 $\mathrm{MPa}^{*} \mathrm{~m}^{1 / 2}$ ). This increase was attributed to pullout of SiC grains in the fracture surfaces indicating weakened bonding between the $\mathrm{SiC}$ and $\mathrm{TaC}$ grains favoring interface debonding and crack deflection resulting in improved strength. In another study [46], they reported that the weakened bonding was a result of thermal expansion mismatch between $\mathrm{TaC}\left(6.3 \times 10^{-6} / \mathrm{K}\right)$ and $\operatorname{SiC}\left(4.3 \times 10^{-6} / \mathrm{K}\right)$ resulting in the thermal residual stresses.

Another form of $\mathrm{SiC}$ reinforcement to UHTCs is the addition of $\mathrm{SiC}$ whiskers and fibers which show a greater toughening benefit compared to the addition of SiC powder. Guicciardi et al. [47] produced $\mathrm{ZrB}_{2}$ ceramic composites with 20 vol.\% SiC whiskers as well as SiC chopped fibers. Figure 13 shows SEM images of the polished cross sections in the directions parallel and normal to the applied pressure during SPS processing. An oriented alignment is seen which is similar to what is seen with the addition of GNP which will be discussed in detail in later sections. With the addition of the SiC fibers, the fracture toughness increased $47 \%$ (from 3.75 to $5.53 \mathrm{MPa}^{*} \mathrm{~m}^{1 / 2}$ ) over the monolithic $\mathrm{ZrB}_{2}$, and the high temperature flexural strength measured at $1200^{\circ} \mathrm{C}$ increased $86 \%$ (from 240 to $447 \mathrm{MPa}$ ). 

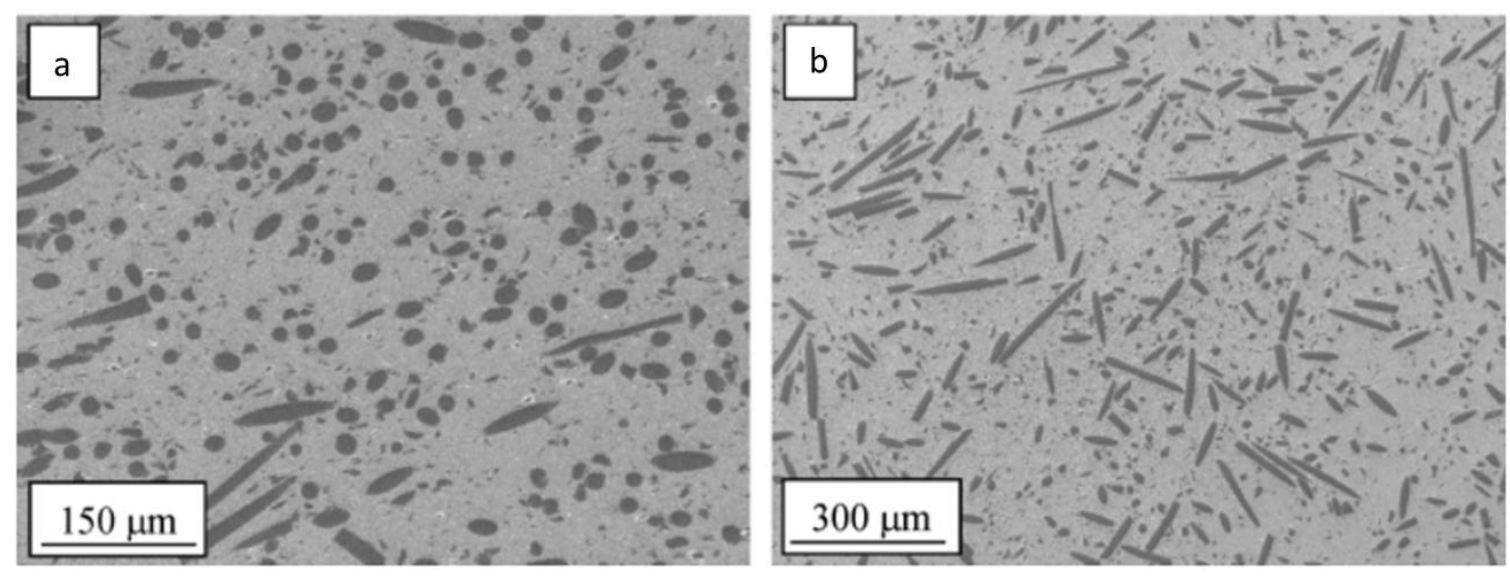

Figure 13: (a) Polished section parallel to the direction of applied pressure, (b) Polished section normal to the direction of applied pressure [47].

When SiC whiskers were added, the fracture toughness increased by 53\% (3.75 to 5.73 $\mathrm{MPa}^{*} \mathrm{~m}^{1 / 2}$ ) over the monolithic $\mathrm{ZrB}_{2}$ and the flexural strength increased by $71 \%$ (from 240 to $411 \mathrm{MPa}$ ). Figure 14 shows the crack propagation during Vickers hardness experiments. With increasing content from 0 to 20 vol.\% SiC whisker addition, the tortuosity (change in direction) of the crack is increased. 

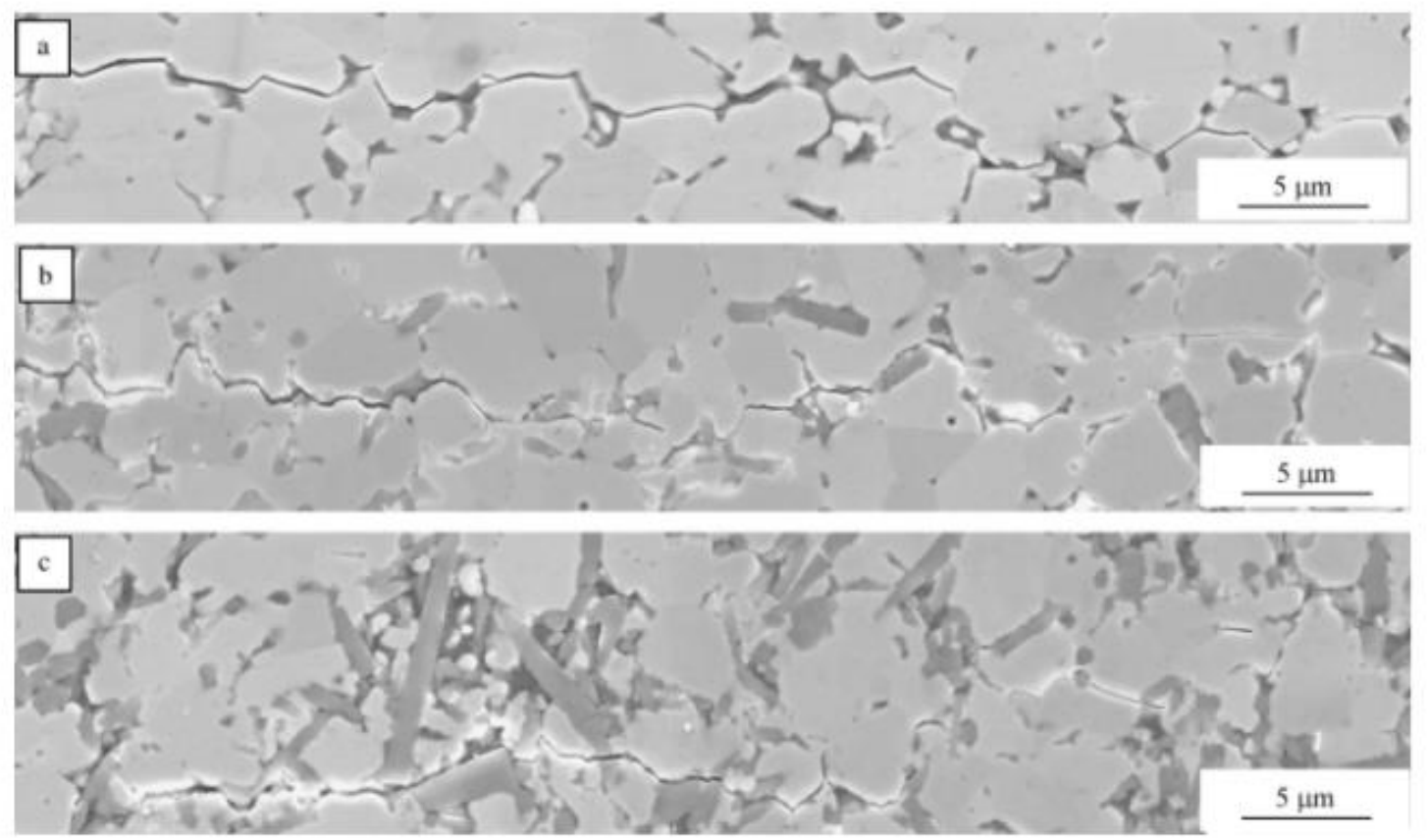

Figure 14: Examples of crack path in (a) baseline material, (b) Z10w and (c) Z20w showing increasing tortuosity of the crack profile with the increase of whisker amount [47].

The increased toughness of the $\mathrm{SiC}$ reinforced ceramics was attributed to crack deflection, crack pinning, and thermal residual stresses between the $\mathrm{SiC}$ and matrix phases.

\subsubsection{Carbon Nanotube Reinforcement of UHTC Composites}

Carbon nanotubes (CNT) have been investigated as reinforcement to a variety of ceramic composites including hydroxyapatite (HA) and TaC. Carbon nanotubes come in a variety of types and sizes. Single-walled carbon nanotubes (SWNT) consist of a single rolled-up graphene sheet whereas multi-walled carbon nanotubes (MWNT) are made up of 2 or more rolls of graphene. The nanotubes are further defined as either long (10-20 micron) or short (1-3 micron) in length. The arrangement of the carbon atoms of the CNTs are responsible for their unique electrical, thermal, and mechanical properties [48]. 
The mechanical properties that make them a candidate for ceramic matrix reinforcement include enhanced tensile strength, elastic modulus, wear resistance, and corrosion resistance. The addition of CNTs to ceramic matrices also enhance sintering and densification due to the increased interparticle diffusion as a result of the high thermal and electrical conductivity of the CNTs between the ceramic grains [49, 50].

The extent of improvement in strength of composites reinforced with CNTs will depend on the type of CNT used. Longer CNTs have a greater surface area that allows a greater load to be transferred between the matrix and CNTs. Bakshi et al. [49] prepared TaC composites with the addition of 4 wt.\% short and long CNTs. The resulting properties are shown in Table 4.

Table 4: Properties of TaC and TaC-4 wt.\% CNT compacts prepared by SPS [49].

\begin{tabular}{|c|c|c|c|c|c|c|c|}
\hline \multirow[t]{2}{*}{ Powder } & \multirow[t]{2}{*}{ SPS compact } & \multicolumn{2}{|c|}{ Density $\left(\mathrm{g} \mathrm{cm}^{-3}\right)$} & \multirow[t]{2}{*}{ Grain size $(\mu \mathrm{m})$} & \multirow{2}{*}{$\begin{array}{l}\text { Nano-hardness } \\
\text { (GPa) }\end{array}$} & \multirow{2}{*}{$\begin{array}{l}\text { Elastic modulus } \\
\text { (GPa) }\end{array}$} & \multirow{2}{*}{$\begin{array}{l}\text { Relative fracture } \\
\text { toughness }\end{array}$} \\
\hline & & Actual & Relative (X) & & & & \\
\hline \multirow{3}{*}{$\mathrm{TaC}$} & $\mathrm{TaC}-100$ & 13.0 & 89 & $0.56 \pm 0.12$ & $17.8 \pm 3.2$ & $335 \pm 28$ & a \\
\hline & $\mathrm{TaC}-255$ & 14.6 & 100 & $4.93 \pm 1.99$ & $26.3 \pm 2.7$ & $469 \pm 46$ & $1^{\mathrm{b}}$ \\
\hline & $\mathrm{TaC}-363$ & 14.6 & 100 & $5.6 \pm 2.4$ & $25.7 \pm 5.7$ & $457 \pm 39$ & $0.99 \pm 0.09$ \\
\hline \multirow{3}{*}{$\begin{array}{l}\text { TaC-4 wt.\% long } \\
\text { CNTs }\end{array}$} & TaC-LC-100 & 11.3 & 95 & $0.61 \pm 0.27$ & $22.9 \pm 2.1$ & $395 \pm 17$ & a \\
\hline & TaC-LC-255 & 11.1 & 94 & $1.06 \pm 0.34$ & $14.2 \pm 1.8$ & $288 \pm 29$ & $1.58 \pm 0.04$ \\
\hline & TaC-LC-363 & 12.3 & 104 & $1.22 \pm 0.43$ & $12.7 \pm 0.7$ & $369 \pm 18$ & $1.60 \pm 0.12$ \\
\hline Spray dried & TaC-SC-100 & 11.1 & 94 & $1.85 \pm 0.72$ & $17.5 \pm 2.4$ & $331 \pm 47$ & a \\
\hline TaC- 4 wt.\% short & TaC-SC-255 & 11.8 & 100 & $1.64 \pm 0.38$ & $14.7 \pm 2.5$ & $313 \pm 37$ & $1.44 \pm 0.21$ \\
\hline CNTs & TaC-SC-363 & 12.3 & 104 & $1.65 \pm 0.44$ & $10.6 \pm 1.2$ & $258 \pm 14$ & $1.08 \pm 0.18$ \\
\hline
\end{tabular}

While the short CNTs are more effective in increasing the densification, long CNTs were better grain growth inhibitors. Although the addition of both short and long CNTs showed an increase in toughening, a greater toughening benefit was observed with the addition of long CNTs over short ones. At pressures greater than $100 \mathrm{MPa}$, destruction of the CNT structure was observed that resulted in graphitic structures. 
The same destruction of CNT structure was seen by Lahiri et al. [51] who also used SPS to consolidate $\mathrm{TaC}$ composites with the addition of 4 wt.\% long and short CNTs. Figure 15 shows that long CNTs retain more of their tubular structure whereas the short CNTs have been reduced to thin platelet-type morphologies similar to GNPs.
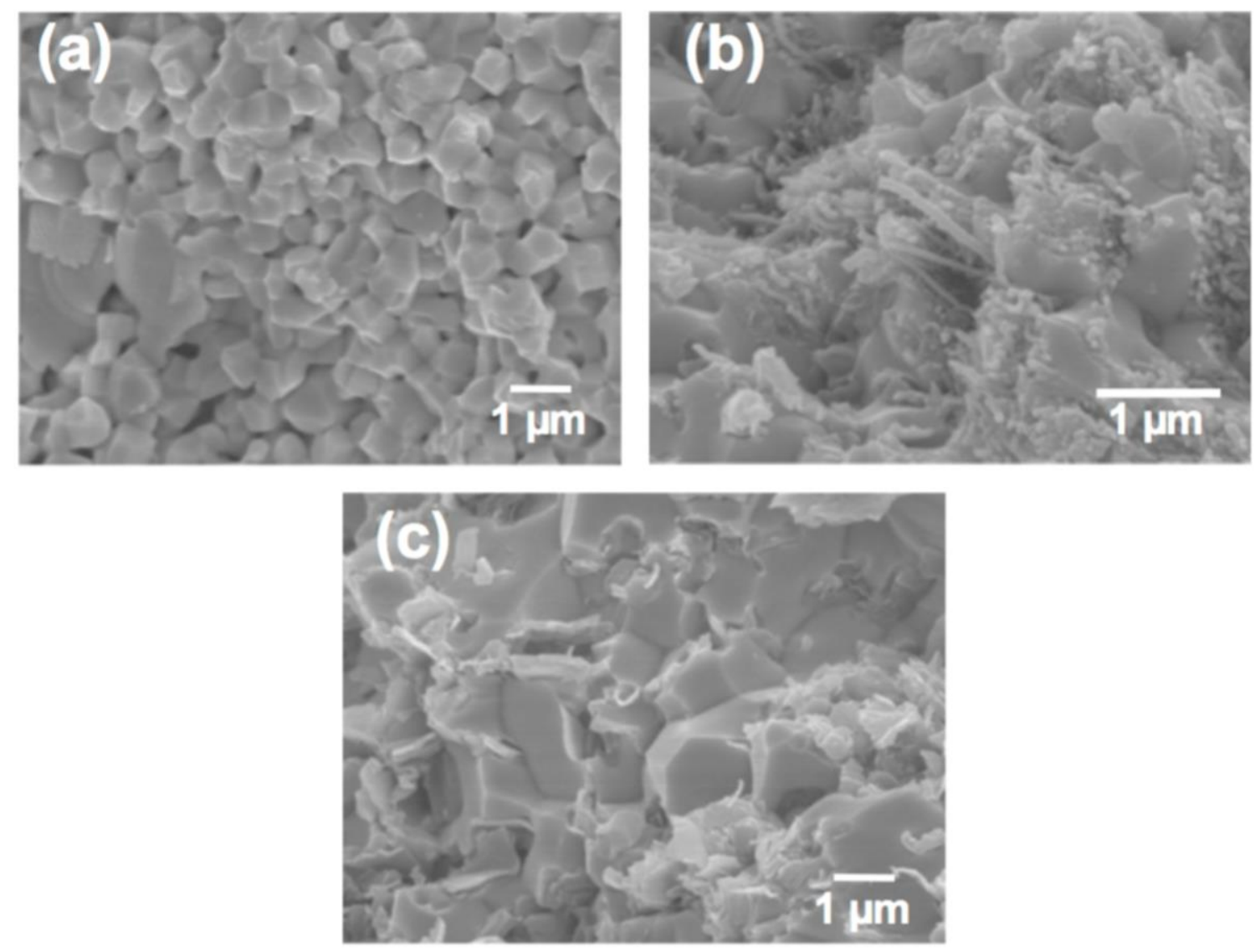

Figure 15: SEM micrograph of fracture surfaces for (a) TaC, (b) TaC-LC, and (c) TaC$\mathrm{SC}[51]$.

Although the elastic modulus and nanohardness were lower with the addition of short CNTs that turned to platelets, the rupture strength was higher (368 MPa) compared with the longer CNTs (279 MPa) that retained the majority of their tubular structure. 
Due to the difficulty in dispersion of CNTs in a composite matrix because of their high propensity to entanglement and the difficulty in retaining their structure, other forms of carbon reinforcement such as GNP show better promise.

\subsubsection{Graphene and Graphene Nanoplatelet Reinforcement of Composites}

Graphene is a two-dimensional, single atom thick sheet of $\mathrm{sp}^{2}$ bonded carbon atoms that is known for its extraordinary thermal, mechanical, and electrical properties [9]. There are four methods that are currently used for the production of graphene [52]: 1) chemical vapor deposition [53, 54], 2) epitaxial growth of graphene films on electrically insulating substrates [55], 3) mechanical exfoliation from bulk graphite [56], and 4) reduction of graphene from graphene oxide [57]. Of the different production methods, the reduction of graphene from graphene oxide provides for large volumes of graphene to be made at a relatively low cost. Graphene nanoplatelets (GNP) are made up of multiple layers of graphene held together by weak van der Waals forces. GNP are easier and less expensive to form while retaining much of the desired mechanical, thermal, and electrical properties of graphene $[15,16]$.

In comparison to CNTs, graphene nanoplatelets have been shown to be superior in reinforcing composites in several ways. Although both GNP and CNTs are similarly made of bonded carbon atoms, the 2D geometry of the GNPs provides greater strengthening and toughening to a composite as a result of the increased surface area as well as the retention of the structure after high temperature processing. Rafiee et al. [12] compared the enhancement of mechanical properties with the addition of $0.1 \mathrm{wt} . \%$ of SWNT, MWNT, and GNP to epoxy nanocomposites. The increase in Young's modulus 
of the GNP reinforced nanocomposite was $31 \%$ greater than the pure epoxy as compared to only a 3\% increase for SWNT addition. The tensile strength of the epoxy saw a $40 \%$ increase with the addition of GNP, whereas an increase of only $14 \%$ was seen with MWNT addition. The fracture toughness of the nanocomposite achieved an increase of $53 \%$ with the addition of GNP while the increase due to the addition of MWNT was only 20\%. The enhanced mechanical properties when using GNP over CNTs was attributed to the GNPs high specific surface area, enhanced nanofiller-matrix adhesion/interlocking due to the wrinkled GNP surface, as well as the 2D geometry of the GNP.

Reinforcement of ceramic composites using GNP benefits the mechanical properties of the bulk composite via three main methods: improvement to the densification and microstructure during processing, improvement of toughness due to the interface stress transfer between the GNP and the matrix, and improvement of toughness due to the mechanical properties and intrinsic deformation mechanisms of the GNP itself. These methods will be discussed in detail in the following section.

\subsection{Reinforcement Mechanisms of GNP in Composites}

As previously stated, the increased toughening in composites reinforced with GNP can be attributed to three different regimes; (1) property changes during processing, (2) increased load capacity prior to initial crack propagation, and (3) crack propagation suppression mechanisms. In the following subsections, the three regimes of increased toughening as well as the anisotropy in the toughening mechanisms will be discussed in detail. 


\subsubsection{Regime 1: Property Changes During Processing}

Graphene reinforcement to composites has the ability to increase densification and homogeneity in the bulk structure of spark plasma sintered composites. The high thermal conductivity of graphene $\left(3,000-5,100 \mathrm{~W}^{*} \mathrm{~m}^{-1} * \mathrm{~K}^{-1}\right)[58,59]$ improves the thermal conductivity of the composite being reinforced leading to more uniform heating during processing. With increasing volume percentages (1, 3, and 5 vol.\%) of GNP added to $\mathrm{TaC}$ before consolidation by spark plasma sintering at $1850^{\circ} \mathrm{C}$, both an increase in relative densification and a decrease in grain growth (up to 68\%) was observed (Table 5) [18].

Table 5: Summary of TaC-GNP samples [18].

\begin{tabular}{|c|c|c|c|}
\hline Sample & $\begin{array}{c}\text { GNP Content } \\
(\mathbf{v o l .} \%)\end{array}$ & $\begin{array}{c}\text { Relative Density } \\
(\boldsymbol{\%})\end{array}$ & Grain Size $(\boldsymbol{\mu m})$ \\
\hline $\mathrm{TaC}$ & None & $94.4 \pm 0.7$ & $4.5 \pm 0.9$ \\
\hline $\mathrm{TaC}-1 \mathrm{G}$ & 1 & $96.9 \pm 0.4$ & $3.4 \pm 1.6$ \\
\hline $\mathrm{TaC}-3 \mathrm{G}$ & 3 & $97.5 \pm 0.4$ & $1.4 \pm 0.6$ \\
\hline $\mathrm{TaC}-5 \mathrm{G}$ & 5 & $98.8 \pm 0.2$ & $1.4 \pm 0.7$ \\
\hline
\end{tabular}

One of the intrinsic toughening mechanisms of GNP in reinforced ceramic composites is the wrapping of the ceramic particles by GNP during sintering. This mechanism is believed to pin the grains during sintering to reduce the grain growth occurrence. The wrapping and pinning of the ceramic grains by GNP results in an effective diffusion barrier that hinders grain growth. Also, the high surface area and thermal conductivity of GNP allow for strong interfacial bonding between TaC grains 
and the GNP which minimizes porosity formation. Increasing the GNP content past 5 vol.\% did not further increase the grain refinement. The leveling off of the refinement was possibly due to the dominating effect of further GNP addition in enhancing the heat transfer in comparison to the effect of additional GNP available to wrap around and pin grains [21].

\subsubsection{Regime 2: Increased Load Capacity Prior to Initial Crack Propagation}

Several studies that have used GNP as reinforcement in composites have shown GNP to successfully increase the fracture toughness by absorbing energy that would otherwise result in crack propagation [17-20].

Liu et al. [17] reinforced $\mathrm{Al}_{2} \mathrm{O}_{3}$ with 0.38 vol.\% GNP and consolidated the composites by SPS. Fracture surfaces of the composites revealed that the GNPs were either distributed between the ceramic grain boundaries as shown in Figure 16a-c or embedded within the ceramic grains as shown in Figure 16d. 

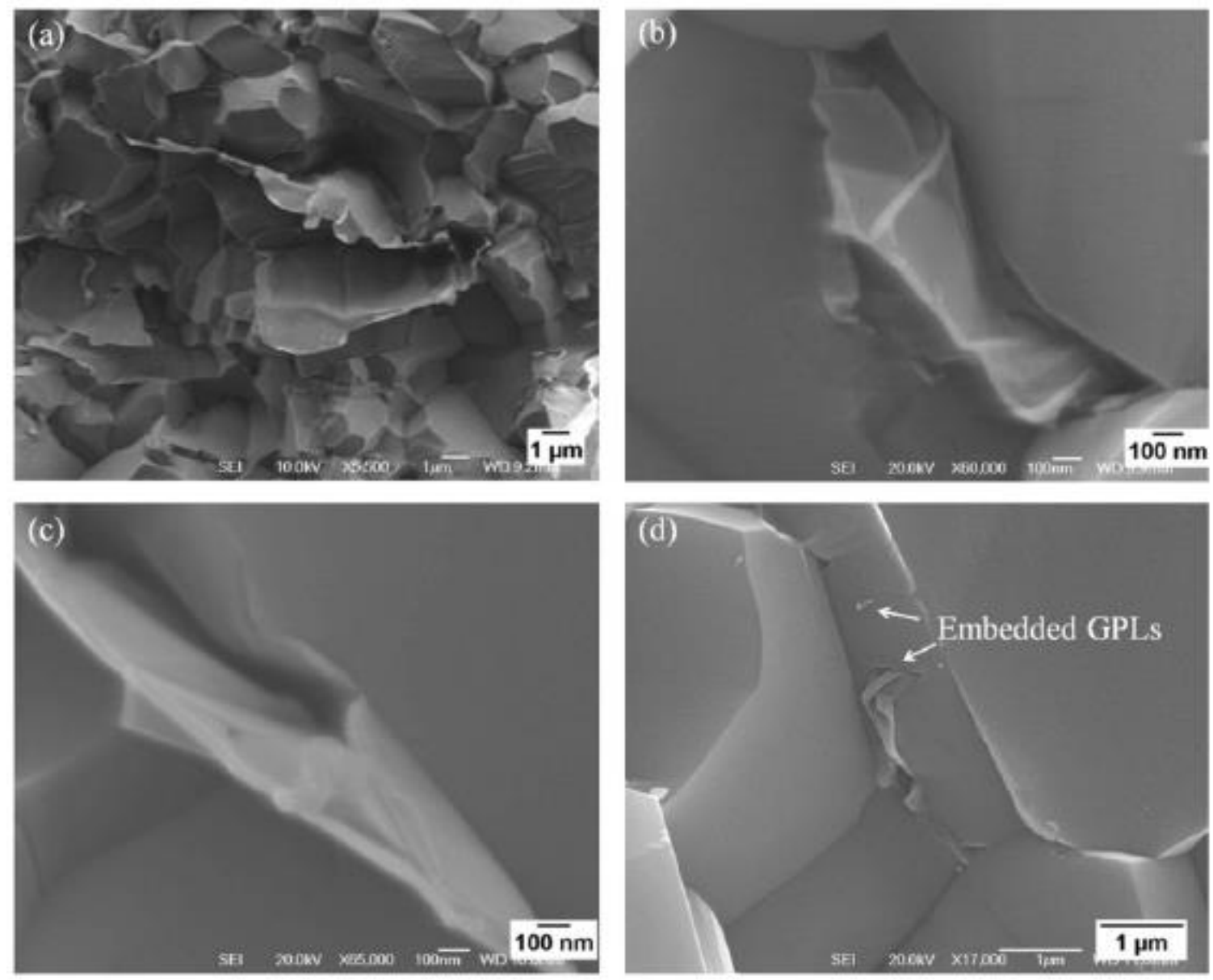

Figure 16: SEM images of fracture surface of GPL-reinforced $\mathrm{Al}_{2} \mathrm{O}_{3}$ composite showing the toughening and reinforcing mechanisms [17].

During consolidation, it was expected that the GNP conformed with the force applied by their neighboring matrix grains to become bent and embedded between the grains. The close contact between the matrix grains and GNP enabled the platelets to anchor at and bind with matrix grains, resulting in increased contact area. They believe that the fracture toughness of the ceramics is therefore improved due to the interfacial friction in the interface between the GNP and ceramic matrix as the energy needed to pull out a GNP is expected to be very high. With the small 0.38 vol.\% GNP addition, the fracture toughness 
of the composite increased by $27 \%$ (from 3.53 to $4.49 \mathrm{MPa}^{*} \mathrm{~m}^{1 / 2}$ ) and the flexural strength increased by $31 \%$ (from 400 to $523 \mathrm{MPa}$ ).

Zhang et al. [19] studied the interfacial behavior of graphene nanosheet reinforced hydroxyapatite (HA) ceramic composites. They found that when the shear stress applied to the interface is larger than the interfacial bond strength, then interfacial delamination occurs as depicted in Figure 17a by the curled graphene nanosheet separating from the HA matrix. If the applied stress is too great, the graphene nanosheet can rupture as shown in Figure 17b. In both of these instances, the energy required to cause these occurrences is being dissipated instead of going towards the formation of cracks.
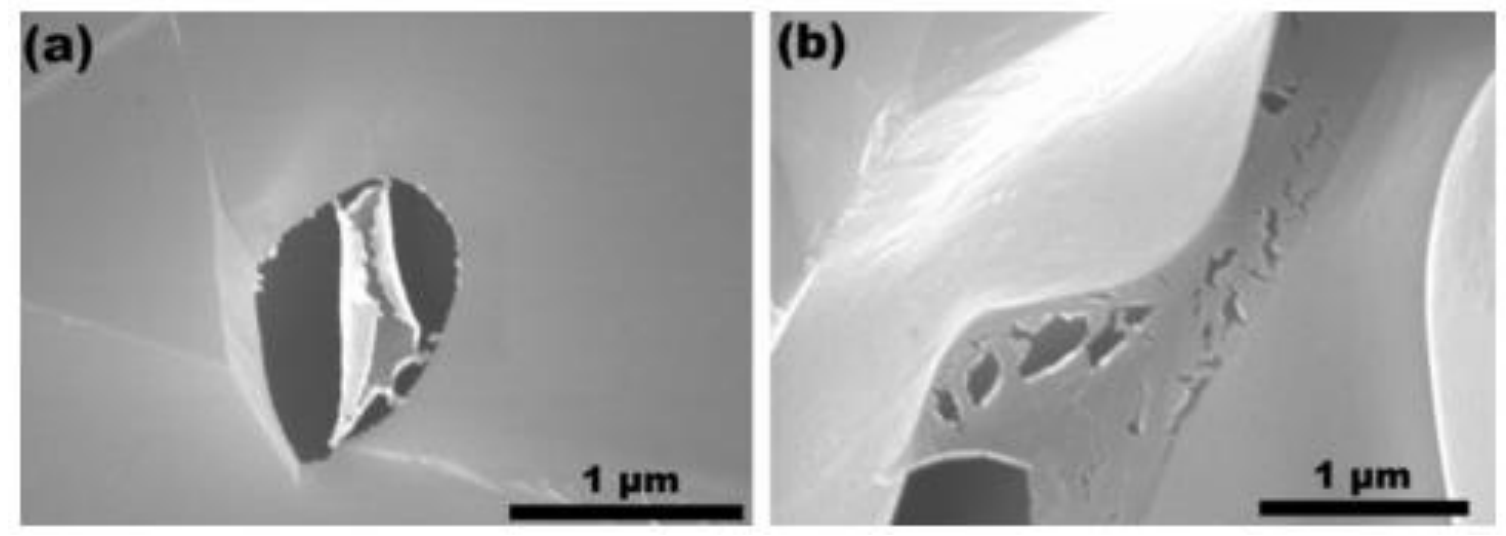

Figure 17: SEM images showing (a) delamination of a GNS-HA interface and (b) a GNS rupture in a GNS/HA composite [19].

The same GNP mechanisms that increased the densification of the GNP reinforced TaC ceramic composites by Nieto et al. [21] in the previous section also work to increase the toughness of the composite by increasing the load capacity prior to crack initiation. In pure $\mathrm{TaC}$, an individual grain is held and bound by other $\mathrm{TaC}$ grains adjacent and above it. In the TaC-GNP composites, they state that GNP between the TaC grains can cause increased displacement of the TaC grain in two modes. First, the GNP 
between $\mathrm{TaC}$ grains acts as a lubricating phase and allows slippage of the grain. And second, due to the bonds between individual graphene sheets in GNP being held together by weak van der Waals forces, the sheets will shear off from the GNP causing the TaC grain to slip and be further displaced. Both of these displacements help to increase the toughening of the overall composite by dissipating energy that would otherwise lead to crack initiation by providing a degree of damping to the $\mathrm{TaC}$ matrix [18]. They also reported three toughening mechanisms (sheet bending, kinking, and sliding) which were shown to be inherent to GNP. Figure 18a shows the bending and kinking of the GNP which is noticed in between grains or embedded in grains. Figure $18 \mathrm{~b}$ shows the sliding of the GNP which occurs during high pressure processing but is also expected to occur during load bearing.
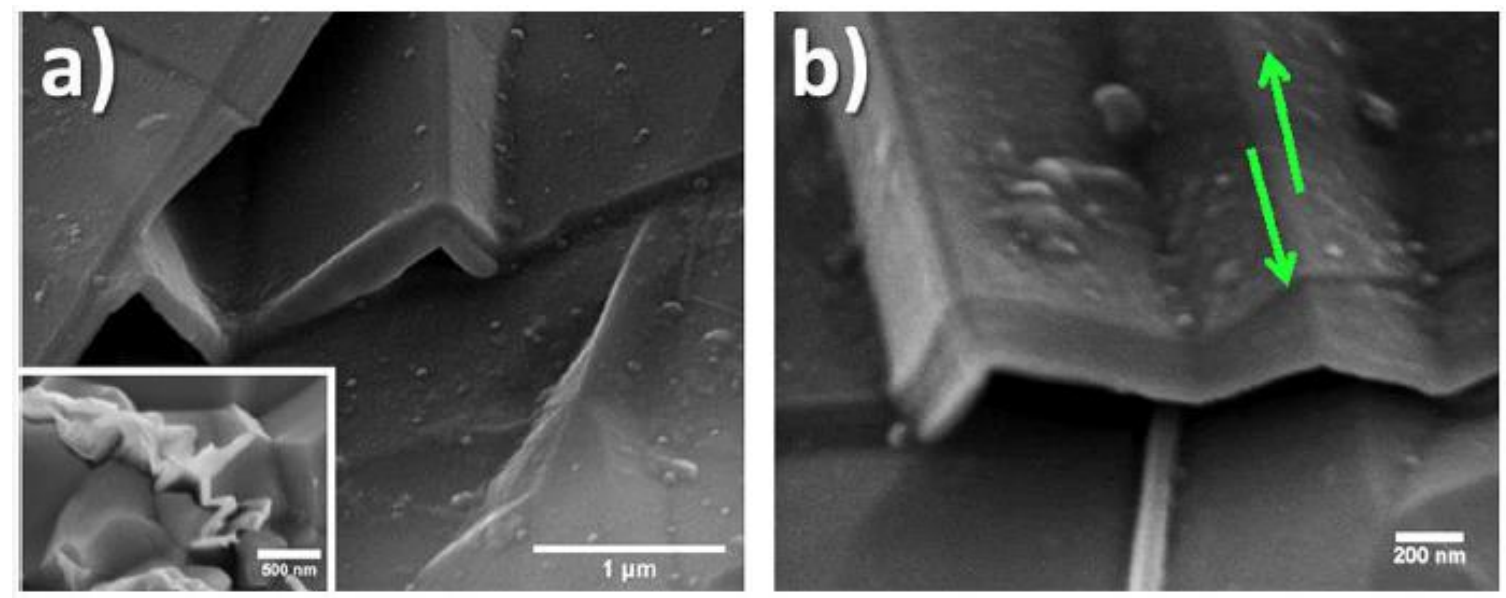

Figure 18: GNP toughening mechanisms in TaC: a) GNP bending and kinking, b) GNP sliding [18].

These bending, kinking, and sliding mechanisms of GNP require strain energy that would otherwise be used for crack formation and propagation [21]. By adding 5 vol.\% GNP to $\mathrm{TaC}$, the fracture toughness increased $98 \%$ (from 5.6 to $11.1 \mathrm{MPa}^{*} \mathrm{~m}^{1 / 2}$ ). 


\subsubsection{Regime 3: Crack Propagation Suppression Mechanisms}

Once crack initiation has taken place, GNP can act to suppress the crack propagation by intrinsic deformation mechanisms such as crack bridging and crack deflection $[20,21,60,61]$. Walker et al. [60] used SPS to sinter $\mathrm{Si}_{3} \mathrm{~N}_{4}$ with GNP ceramic composites and found that with the addition of just 1.5 vol.\% GNP, the fracture toughness increased $235 \%$ from 2.8 to $6.6 \mathrm{MPa}^{*} \mathrm{~m}^{1 / 2}$. Once crack propagation was initiated using Vickers indentation, the crack was seen to deflect and branch when coming into contact with GNP dispersed throughout the matrix (Figure 19a). 


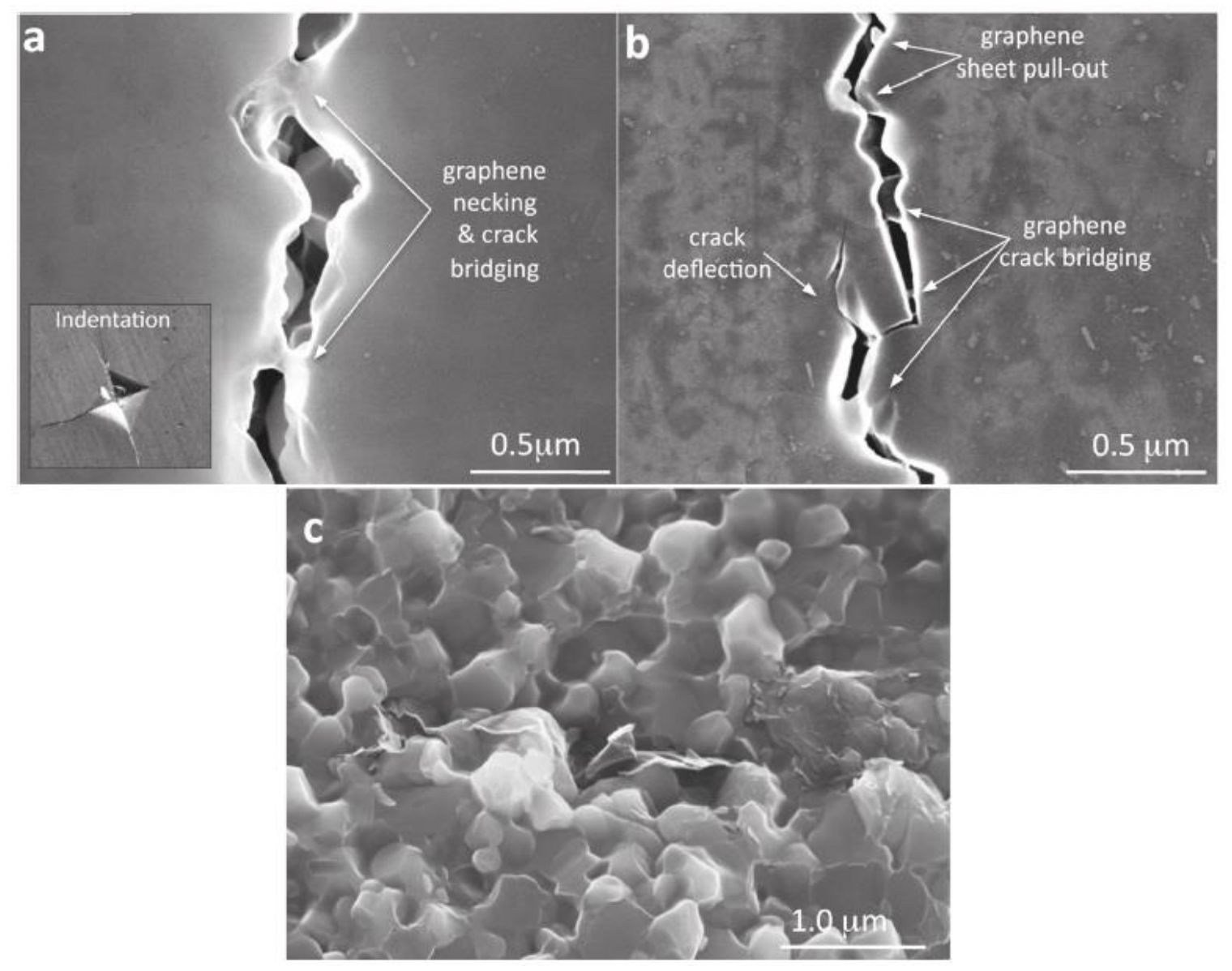

Figure 19: Toughening mechanisms in GPL-Si $\mathrm{N}_{4}$ nanocomposites. (a) Microhardness testing resulting in the creation of radial cracks stemming from the microhardness indent (inset image). Closer examination of the radial cracks reveals GPL bridging the crack at several locations, two of which are shown in this high-resolution SEM image. (b) Further examination of the radial cracks indicates that they follow a tortuous crack propagation path. (c) Fracture surface of the bulk sample indicates the presence of three-dimensional toughening mechanisms for the GPL-Si ${ }_{3} \mathrm{~N}_{4}$ nanocomposite [60].

When zooming in on the cracks, they were able to find evidence of sheet pull-out and graphene sheets bridging the cracks (Figure 19a-b). Also in Figure 19a, there are two regions within the crack where it appears that the GPLs are necking down to a smaller cross-sectional area within the crack wake. They believe that the 2D structure of the GNP, seen by the GNP protruding out of the fracture surface of Figure 19c, explains the ability of the GNP to block the in-plane propagation of the crack. When the in-plane 
propagation is blocked, the crack is forced to change directions (to an out-of-plane direction) and suggests that the 2D structure of the GNP promotes the deflection of cracks in three dimensions. All of these toughening mechanisms that were seen in this GNP reinforced composite act to dissipate the forces that would be used for increased cracking, thereby enhancing the overall fracture toughness of the composite. While the crack bridging is seen in CNT reinforced composites, the structure of the GNP provides the additional toughening mechanism of forcing a change in crack direction which has not been seen in ceramic particulate, CNT, or fiber reinforced ceramics.

Kvetková et al. [61] also saw the same toughening mechanisms (crack bridging, crack deflection, and GNP pull-out) in a ceramic composite reinforced with GNP. They found that when the propagating crack comes into contact with smaller sized GNPs, then crack branching with secondary crack lengths of several microns is very frequently observed. When a propagating crack comes into contact with larger GNPs, crack deflection is usually observed as shown in Figure 20a-b. 

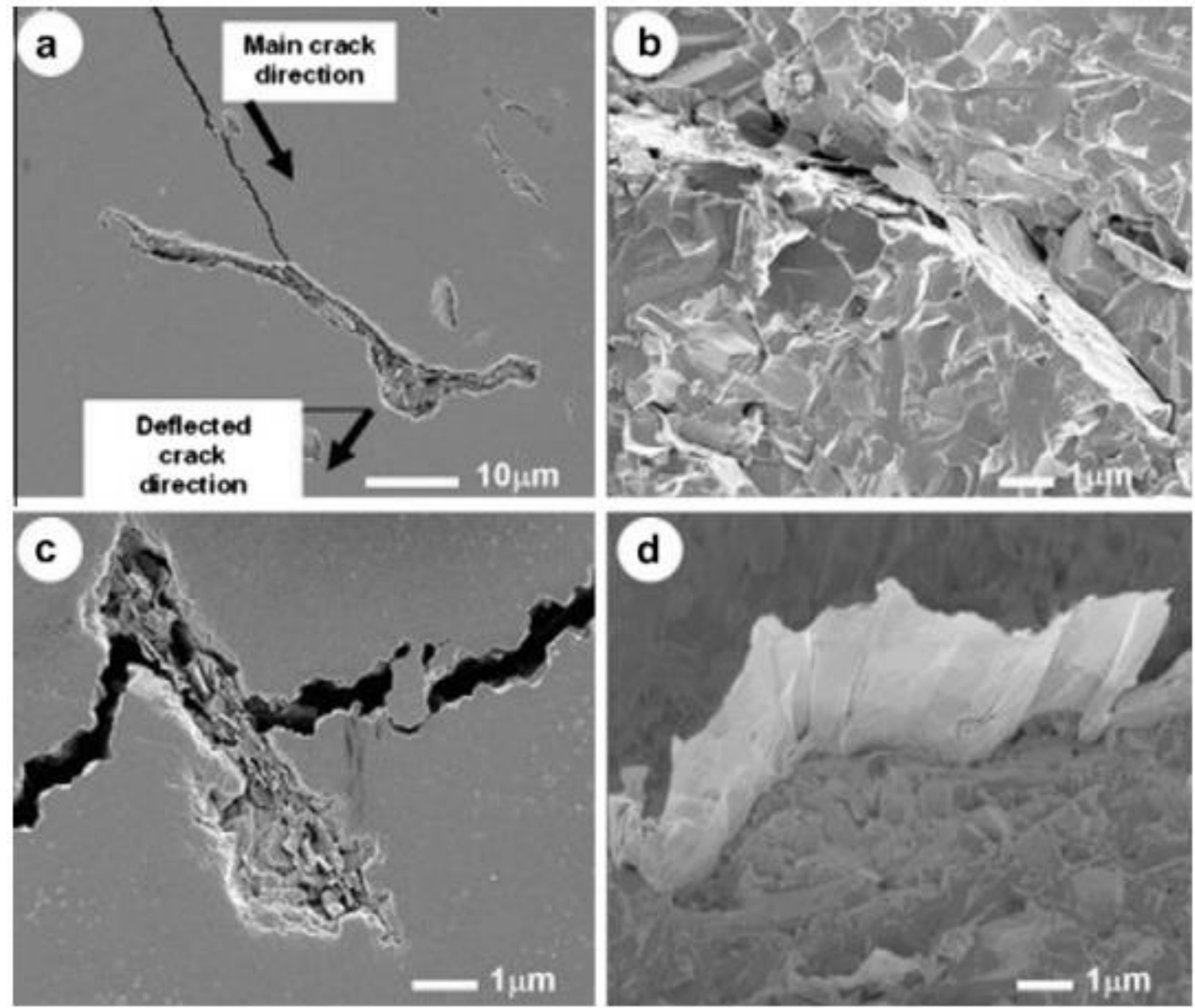

Figure 20: Toughening mechanisms in composites. (a) Crack deflection and slow down of the crack propagation at GPLs (fracture line), (b) crack deflection parallel and perpendicular to the fracture surface, (c) crack bridging by FPLs on the fracture line with the plane of sheet nearly parallel to the plane of the polished surface, (d) crack bridging with a GPL on the fracture surface, with the plane of the sheet perpendicular to the plane of the fracture surface [61].

After the crack was deflected by the larger GNP, the crack was seen to slow down and crack branching was noticed in a different direction to the main crack. Figure 20c shows crack bridging by a large GNP, although crack bridging also occurred when cracks came into contact with small GNPs as well. The pullout of GNPs (Figure 20d) was observed when the GNP plane was perpendicular to the plane of the fracture surface. This pullout occurred after initially bridging the crack in the zone of the crack far behind the crack tip. 
This pullout mechanism is expected to be a greater toughening mechanism in systems reinforced with GNP compared to systems reinforced with CNTs due to the wrapping of the GNP around the ceramic grains of the composite.

\subsubsection{Anisotropy of GNP Toughening Mechanisms}

The processing of ceramic matrix composites reinforced with GNP by SPS leads to two distinct occurrences. The first is that the GNPs orient themselves perpendicular to the pressing axis of the SPS pressure ram. The second is that the lower sintering temperature along with the fast consolidation process lends to the retention of the GNPs structure after sintering $[18,21,60]$. The orientation of platelet-structured particles has been previously seen during compression in $\mathrm{SiC}$ reinforced $\mathrm{Al}_{2} \mathrm{O}_{3}$ composites [62] as well as in GNP reinforced composites $[21,60,63]$. This alignment and orientation of the GNP will have an effect on the electrical properties of GNP reinforced composites as well as have a possible effect on the toughening mechanisms.

Ramirez et al. [64] studied the electrical conductivity in graphene nanoplatelet/silicon nitride composites using conducting scanning force microscopy. Current maps were obtained for two orientations of the composites, parallel and perpendicular to the press sintering axis, to study the effect of GNP orientation as shown in Figure 21. They found that due to the GNP's aspect ratio and stiffness, the GNPs become self-oriented, lying on their a-b plane, during SPS processing. 

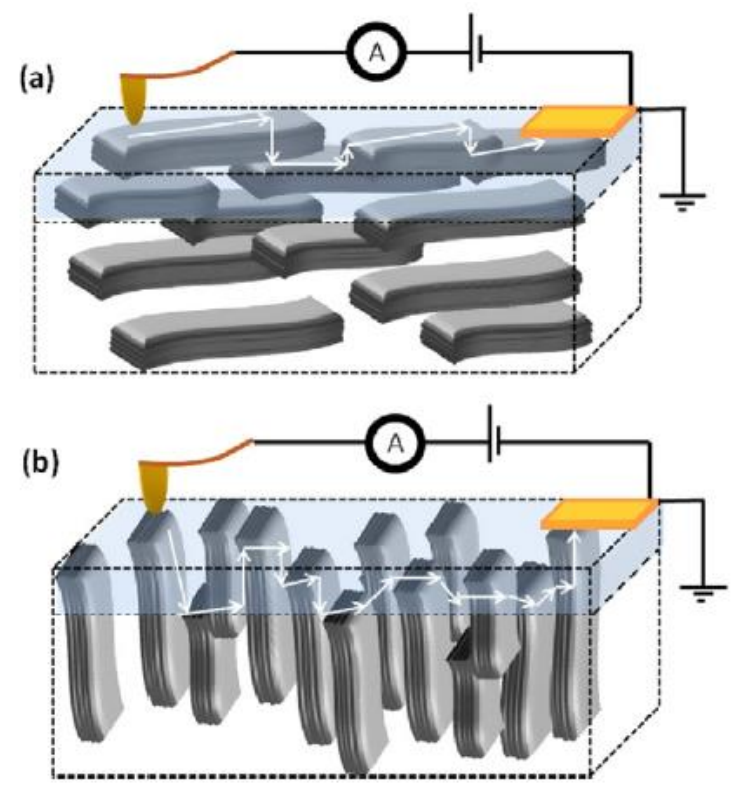

Figure 21: Simplified schematics of the measuring geometry to illustrate the possible conducting paths for the orientations (a) perpendicular and (b) parallel with respect to the SPS pressing axis [64].

When calculated for the two different orientations, it was found that the perpendicular direction resulted in twice the effective current compared to the parallel orientation direction.

In regards to the anisotropy of toughening mechanisms of GNP reinforced composites with respect to orientation, there has not been in-depth study. Dai et al. [65] used computational analysis to model graphene reinforced polymer composites. They generated several 3D unit cell models with 25 graphene sheets with aspect ratio 2000 (graphene fraction of $0.5 \%$ ) and varied orientations (aligned and random) as shown in Figure 22. 

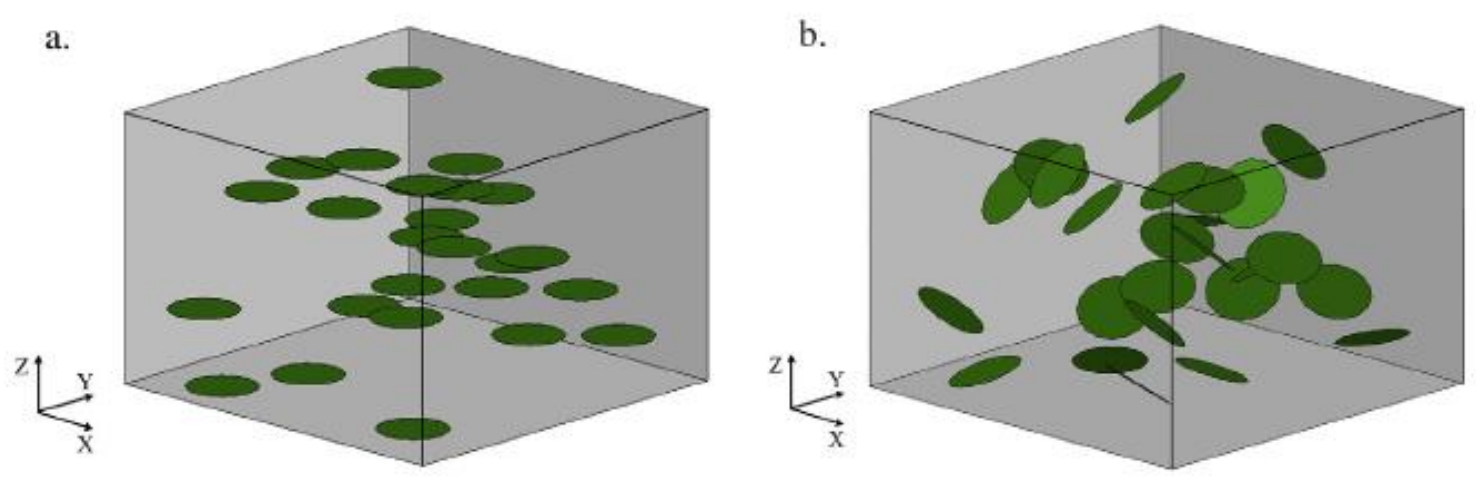

Figure 22: Examples of 3D unit cell models of graphene reinforced composites: (a) Aligned exfoliated model, (b) Random exfoliated model [65].

The composites with aligned graphene sheets demonstrated a higher Young's modulus (3.52 GPa in $\mathrm{X}$ direction and $3.27 \mathrm{GPa}$ in $\mathrm{Z}$ direction versus $1.92 \mathrm{GPa}$ in the $\mathrm{X}$ direction and $1.84 \mathrm{GPa}$ in the $\mathrm{Z}$ direction for the material with random orientations), and higher ultimate strength (106.76 $\mathrm{MPa}$ and $89.56 \mathrm{MPa}$ in $\mathrm{X}$ and $\mathrm{Z}$ direction, respectively, versus 40.91 and $38.82 \mathrm{MPa}$ for the random model). This resulted in the conclusion that the random/misaligned nanosheets lead to a reduction of Young's modulus of the composites by $43.8 \%$ and to a reduction in strength of $56.7 \%$ (along the $\mathrm{Z}$ direction). Next, they modeled crack morphology in unit cells of each orientation. The crack initiates at the graphene-matrix interface region and propagates into the matrix as shown in Figure 23.
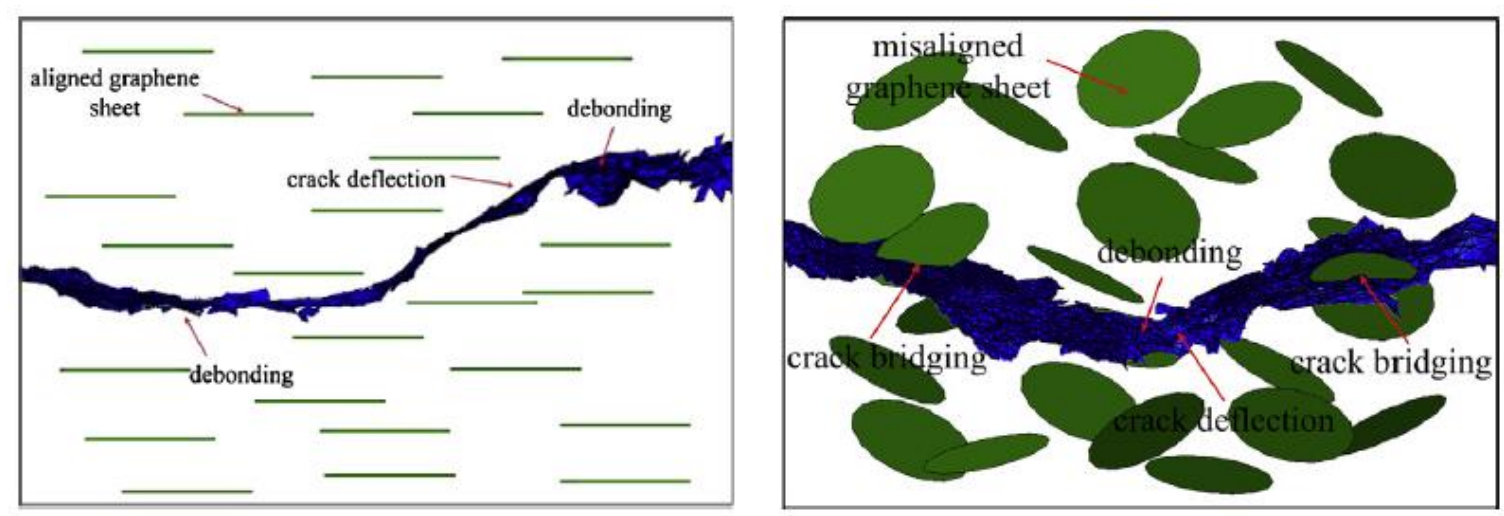

Figure 23: Crack morphology in an aligned and random model [65]. 
It is observed that the main damage/toughening mechanisms in the case of a composite with aligned sheets are debonding and crack deflection. These same mechanisms are seen in the case of randomly oriented graphene sheets along with the additional mechanisms of crack pinning and crack bridging. 


\section{Chapter III: Materials and Methods}

This chapter details the materials used as well as the synthesis of the bulk GNP, TaC-NbC with and without sintering additives, and $\mathrm{TaC}-\mathrm{NbC}$ with sintering additives and GNP addition. The characterization techniques and respective equipment used for obtaining information on the microstructure and mechanical properties is also detailed.

\subsection{Materials}

In this study, pre-alloyed TaC-NbC powder was used as our base ceramic matrix. Graphene nanoplatelets were used as a reinforcing phase to increase the toughness of the TaC-NbC matrix as well as consolidated as a bulk compact to study the anisotropy of the deformation mechanisms. Sintering additives (nano-Si and nano- $\mathrm{B}_{4} \mathrm{C}$ ) were added to the ceramic matrix to enhance the sintering of the ceramic matrix for improved densification. In the following subsections, the characteristics and properties of these materials will be detailed.

\subsubsection{TaC-NbC}

The powder that provided the base for our ceramic matrix was a pre-alloyed Tantalum-Niobium Carbide (TaC-NbC) solid-solution made up of 80 wt.\% TaC and 20 wt.\% $\mathrm{NbC}\left(\left(\mathrm{Ta}_{0.67} \mathrm{Nb}_{0.33}\right) \mathrm{C}\right)$. This powder was manufactured by Inframat Advanced Materials, LLC (Manchester, CT) who gave the purity by weight to be $>99.6 \%$ with an oxygen content $\leq 0.2 \%$ and a free carbon content $\leq 0.15 \%$. The average powder size was measured from an SEM image of the as-received powder (Figure 24) using ImageJ image processing software. Fifty measurements were taken to give an average size of $0.80 \pm$ $0.26 \mu \mathrm{m}$. 


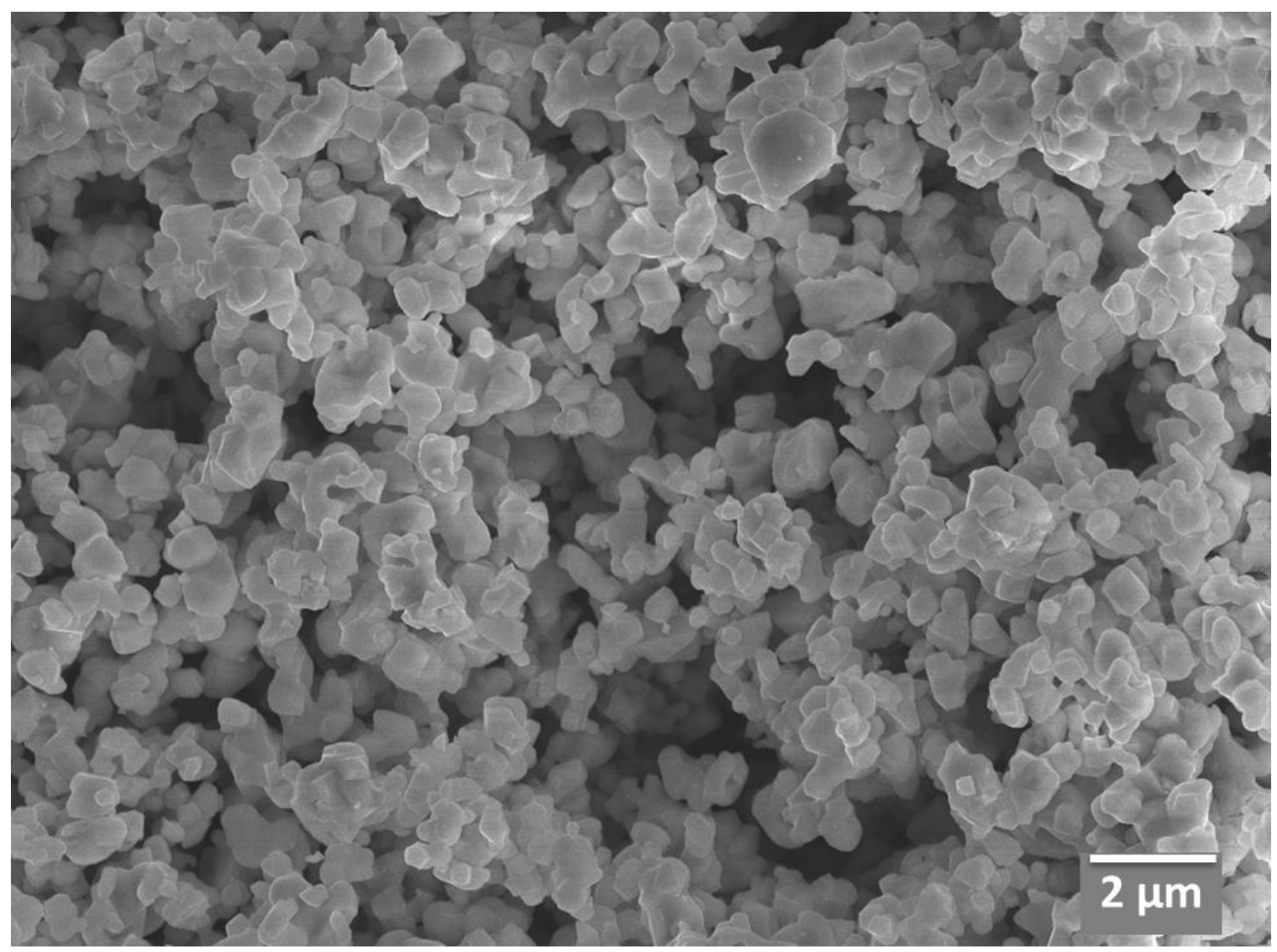

Figure 24: SEM image of the as-received TaC-NbC powder.

\subsubsection{Sintering Additives}

The sintering additives investigated to enhance the sintering of $\mathrm{TaC}-\mathrm{NbC}$ in this research were $\mathrm{B}_{4} \mathrm{C}$ and Silicon nanoparticles. They were chosen based on their high temperature properties when considering the possible secondary phases formed during the sintering process. With the addition of $\mathrm{B}_{4} \mathrm{C}$, secondary phases of $\mathrm{TaB}_{2}$ and $\mathrm{NbB}_{2}$ have the potential to form (Equations 1 and 2) which have melting points of $3037^{\circ} \mathrm{C}$ and $3050^{\circ} \mathrm{C}$, respectively.

$$
\begin{aligned}
& 2 \mathrm{TaC}+\mathrm{B}_{4} \mathrm{C}=2 \mathrm{TaB}_{2}+3 \mathrm{C} \ldots \ldots \ldots \ldots \ldots \text { Equation (1) } \\
& 2 \mathrm{NbC}+\mathrm{B}_{4} \mathrm{C}=2 \mathrm{NbB}_{2}+3 \mathrm{C} \ldots \ldots \ldots \ldots \ldots \text { Equation (2) }
\end{aligned}
$$


With the addition of $\mathrm{Si}$, the formation of $\mathrm{SiC}$ (Equations 3 and 4) is expected which has a melting point of $2830^{\circ} \mathrm{C}$.

$$
\begin{aligned}
& \mathrm{TaC}+3 \mathrm{Si}=\mathrm{TaSi}_{2}+\mathrm{SiC} \ldots \ldots \ldots \ldots \ldots \ldots . . . \ldots \text { Equation }(3) \\
& \mathrm{NbC}+3 \mathrm{Si}=\mathrm{NbSi}_{2}+\mathrm{SiC} \ldots \ldots \ldots \ldots \ldots \ldots \text { Equation (4) }
\end{aligned}
$$

The $\mathrm{B}_{4} \mathrm{C}$ was purchased from US Research Nanomaterials, Inc (Houston, TX) who gave a purity by weight $>99 \%$ with a composition of $77.48 \%$ Boron, $21.52 \%$ Carbon, $<0.08 \%$ Nitrogen, and $<0.1 \%$ each of oxygen, $\mathrm{Si}, \mathrm{Fe}$, and Ni. While the company declared an average particle size of $45-55 \mathrm{~nm}$, SEM imaging of the as-received powder (Figure 25) revealed a bi-modal size distribution with means of $50 \mathrm{~nm}$ and $5 \mu \mathrm{m}$.
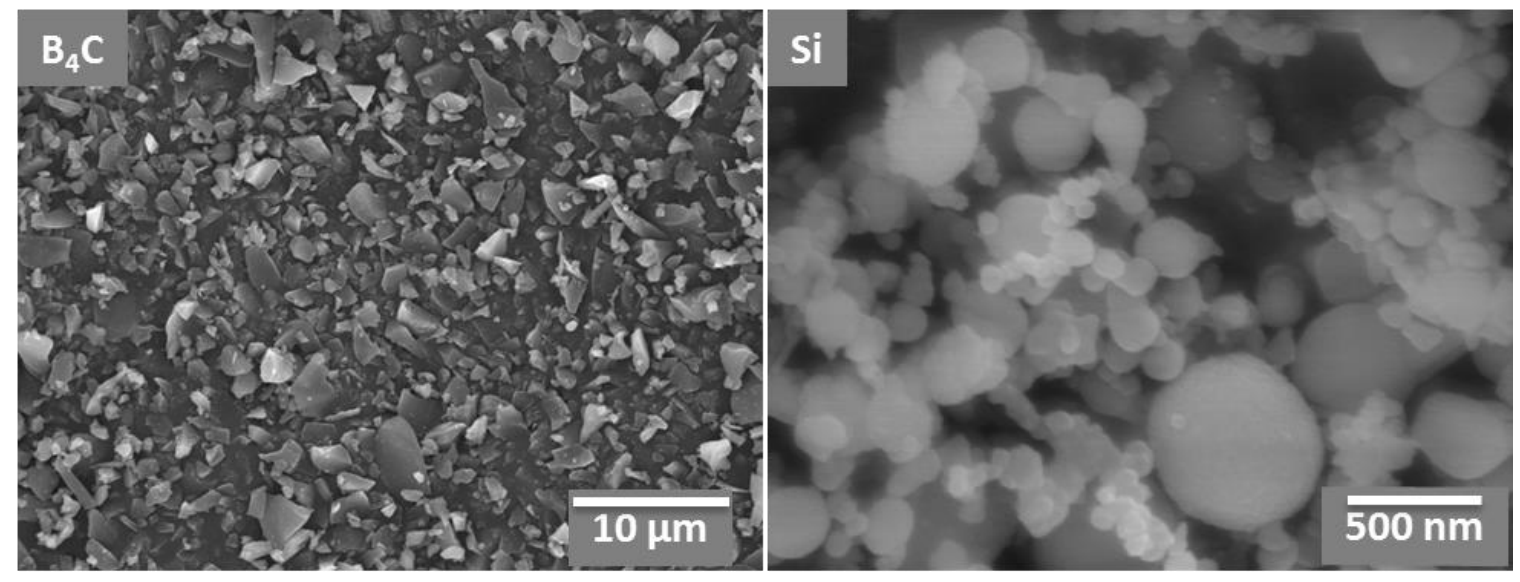

Figure 25: SEM images of $\mathrm{B}_{4} \mathrm{C}$ and $\mathrm{Si}$ powders.

The Silicon nanoparticles were purchased from SkySpring Nanomaterials, Inc. (Houston, TX) who gave a purity by weight $>99 \%$ with a composition of $\mathrm{Si} \geq 99 \%$ and $\mathrm{Cu}<0.45 \%$. The average particle size measured from SEM imaging (Fig. Figure 25) was $100 \mathrm{~nm}$.

Preliminary experimentation was performed with varying volume percentages $(5$ and 10 vol. $\%$ ) of sintering aid addition to determine the optimum amount. For each of the 
sintered samples, the relative density was measured by the water immersion method as well as the Elastic Modulus and Hardness using nano-indentation.

\subsubsection{Graphene Nanoplatelets}

The graphene nanoplatelets (xGNP-M-5) chosen for reinforcement of the ceramic matrix were purchased from XG Sciences (Lansing, MI). The GNP particles have an average diameter of $5 \mu \mathrm{m}$ and a thickness of approximately $6-8 \mathrm{~nm}$, giving a typical surface area of 120 to $150 \mathrm{~m}^{2} / \mathrm{g}$ [66]. Given that each layer of graphene is approximately $0.35-0.37 \mathrm{~nm}$ thick $[15,19]$, the average GNP particle is made up of about 20 sheets of graphene. Figure 26 shows both SEM and TEM images of the graphene nanoplatelets.
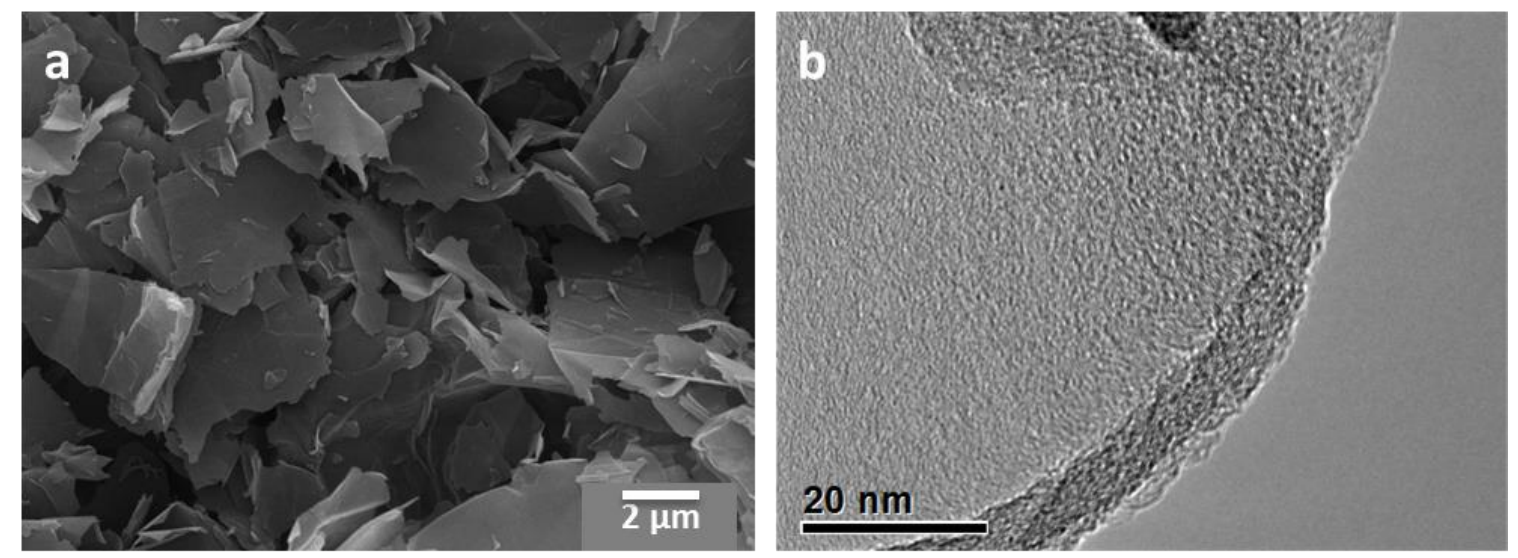

Figure 26: (a) SEM and (b) TEM images of as-received Graphene Nanoplatelets.

In the SEM image, wrinkles and folds are visible on some of the platelets, especially towards the edges. The TEM image shows a GNP with the cross-section of an edge visible that corresponds to a thickness of approximately $8 \mathrm{~nm}$. 


\subsection{Experimental Procedure}

\subsubsection{Powder Preparation Methods}

Initial investigation into powder preparation methods included wet chemistry methods (bath sonication, blending, and tip sonication) as well as a mechanical high energy ball milling method. For each method investigated, a powder mixture of $\mathrm{TaC}-\mathrm{NbC}$ with 7 vol.\% Si addition and 3 vol.\% GNP addition was arbitrarily chosen to compare the dispersion and powder morphology of both sintering additive and reinforcement material. Bath sonication was performed by separating the powder into 4 different beakers in 500 $\mathrm{ml}$ of methanol. Sonication was performed for 90 minutes with sintering aid alone ( $\mathrm{Si}$ ) followed by 90 minutes with the addition of the GNP, and finished with an addition 90 minutes with the addition of $\mathrm{TaC}-\mathrm{NbC}$. The mixtures were then dried overnight in an air atmosphere at $80^{\circ} \mathrm{C}$, broken up with a mortar and pestle, and dried an additional 5 hours to ensure no moisture remained. Blending was performed in a single-blade kitchen blender for a total of 12 minutes in methanol in 1.5 minute increments followed by a 2.5 minute cool down. Ball milling, which has previously been shown to increase the sinterability of samples by reducing the starting powder size, was performed in $25 \mathrm{ml}$ stainless steel canisters with four $10 \mathrm{~mm}$ stainless steel balls for 60 minutes at $1350 \mathrm{RPM}$.

Tip sonication was performed using a Vibra-Cell VCX750 ultrasonic processor (Sonics \& Materials, Inc., Newtown, CT) outfitted with a 3/4 " high gain probe (Figure 27). 


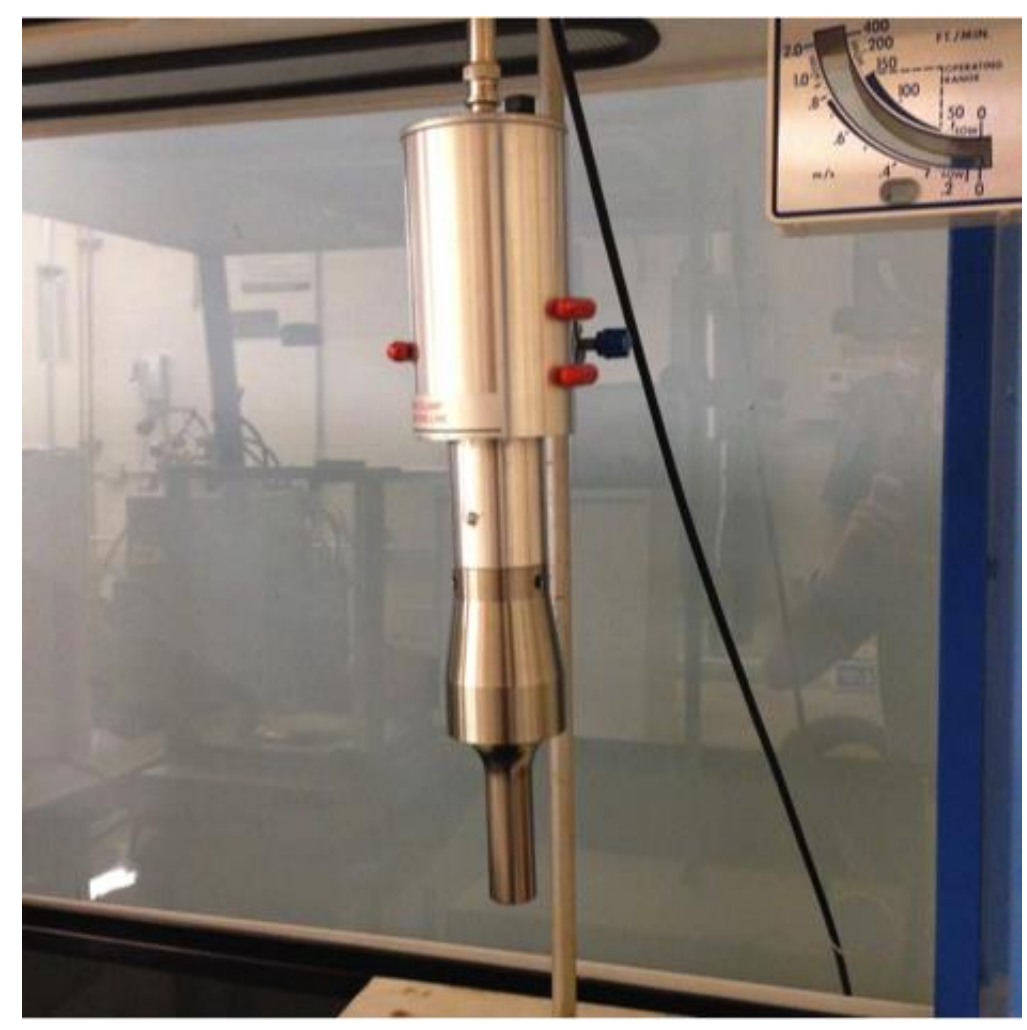

Figure 27: Tip sonication 3/4" high gain probe.

The powder was split up into 3 beakers filled with $400 \mathrm{ml}$ of methanol (when Si was used as the sintering additive) or acetone (when $\mathrm{B}_{4} \mathrm{C}$ was used as the sintering additive) so as to reduce any chance of a reaction with the powder. Each beaker was tip sonicated at 95\% amplitude for 45 minutes. The beakers were surrounded by an ice bath which cooled the mixture to keep the temperature below the boiling points of the acetone and methanol. This was necessary because of the increase in temperature resulting from the high energy imparted to the mixture. Following sonication, the powders were dried overnight in an oven at $80^{\circ} \mathrm{C}$, broken up by a mortar and pestle, and subsequently dried an additional 5 hours to ensure no moisture remained. In order to get sintered samples with a thickness between 4 and $5 \mathrm{~mm}, 16.5$ grams of powder were used in making each compact. The sintered compacts of pure GNP did not undergo any form of pre-sintering processing. 


\subsubsection{Consolidation by Spark Plasma Sintering}

The samples were consolidated using a Thermal Technologies model 10-4 spark plasma sintering machine (Thermal Technologies, LLC, Santa Rosa, CA) (Figure 28).

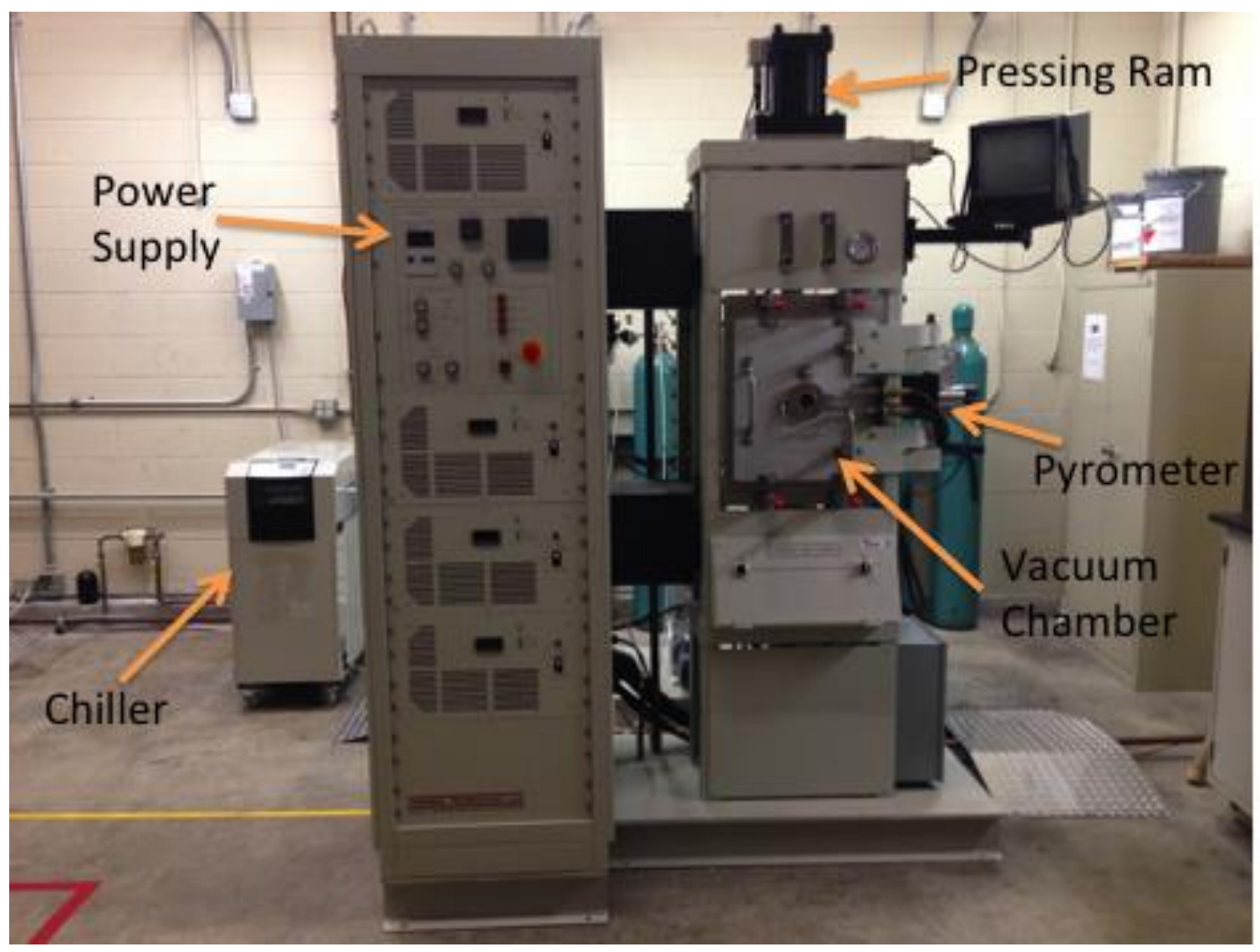

Figure 28: Thermal Technologies Model 10-4 SPS machine installed in the Plasma Forming Lab at FIU.

Consolidation was carried out in a $20 \mathrm{~mm}$ diameter graphite die (Figure 29). Graphite is used due to its high thermal and electrical conductivity. For improved current flow, the starting powder was surrounded by graphite foil which also prevented reactions between the powder and graphite punches and die. Temperature was measured using a pyrometer positioned on the side of the chamber pointing through a pass-through window. The pyrometer was aligned to a hole drilled halfway through the side of the graphite die to 
improve the accuracy of the measured temperature. Each sample was consolidated under vacuum atmosphere $\left(<2 \times 10^{-2}\right.$ Torr $)$.

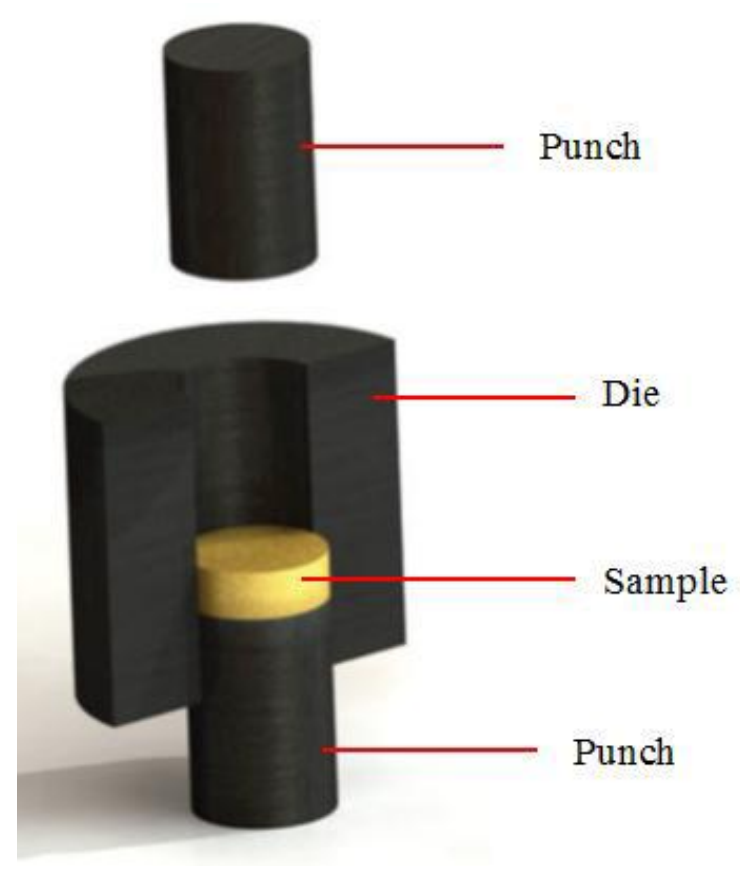

Figure 29: Schematic of the graphite punches and die assembly.

When using SPS, the machine outputs and logs the process parameters during sintering. These parameters, including temperature, ram displacement, chamber pressure, and ram pressure, can be combined into a plot to facilitate analysis of the different stages of sintering. Figure 30 is a representative plot of the SPS process outputs. As an example of the benefit of comparing the processing parameters following sintering, if you take a look at when the slope of the instantaneous relative density begins to drastically increase (regimes 3 and 4 in the figure), the increase in densification is accompanied with an increase in chamber pressure. This increase in chamber pressure can be explained by outgassing that occurs as a result of a reaction taking place. By looking at the temperature 
at which this takes place, the possible reactions that lead to the increase in densification can be narrowed down.

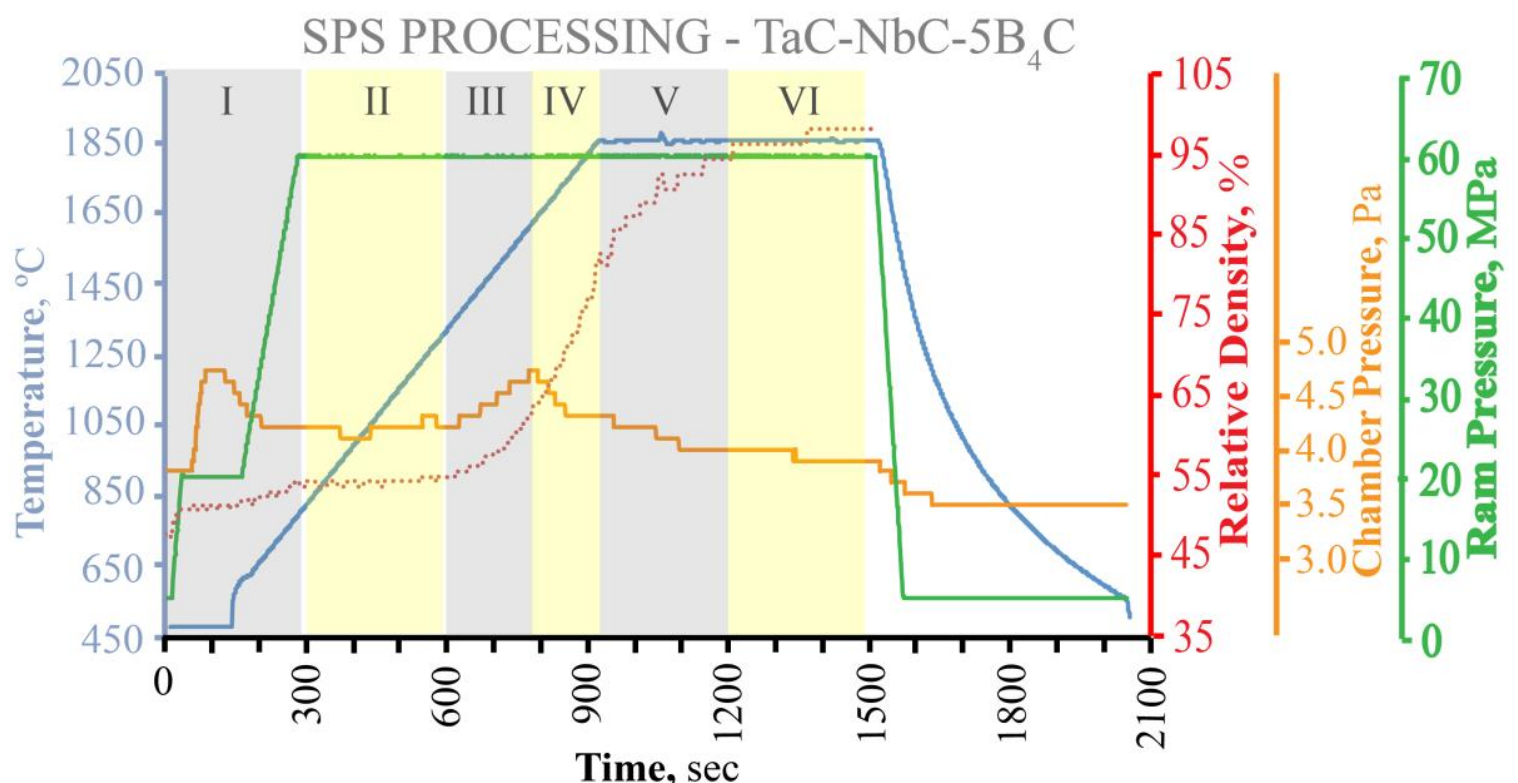

Figure 30: Representative plot of the SPS processing parameter outputs.

In the following subsections, determination of the processing parameters as well as the densification stages will be discussed.

\subsubsection{Initial Evaluation of Processing Parameters}

Ceramic composite samples were initially made using parameters used by our group to consolidate $\mathrm{TaC}$ powder by $\mathrm{SPS}\left(1850^{\circ} \mathrm{C}, 60 \mathrm{MPa}\right.$ pressure, $100^{\circ} \mathrm{C} / \mathrm{min}$. heating rate, and a dwell time of 10 minutes). Experimentation was then performed by varying the maximum temperature, the heating rate, the pressure, and the dwell time and are reported in results section 4.2.3.

For the consolidation of the bulk GNP samples, SPS was carried out using parameters previously used by our group. The samples were consolidated at a 
temperature of $1850^{\circ} \mathrm{C}$, a pressure of $80 \mathrm{MPa}$, with a heating rate of $200^{\circ} \mathrm{C} / \mathrm{min}$. and a dwell time of 10 minutes.

\subsubsection{Evaluation of SPS Densification Stages}

Based on the composition of the powder (which sintering additive was being used), the SPS dwell time was further refined based on when densification was taking place. In order to better understand when densification is taking place, the instantaneous relative density throughout the SPS process is calculated (as shown in Figure 31).

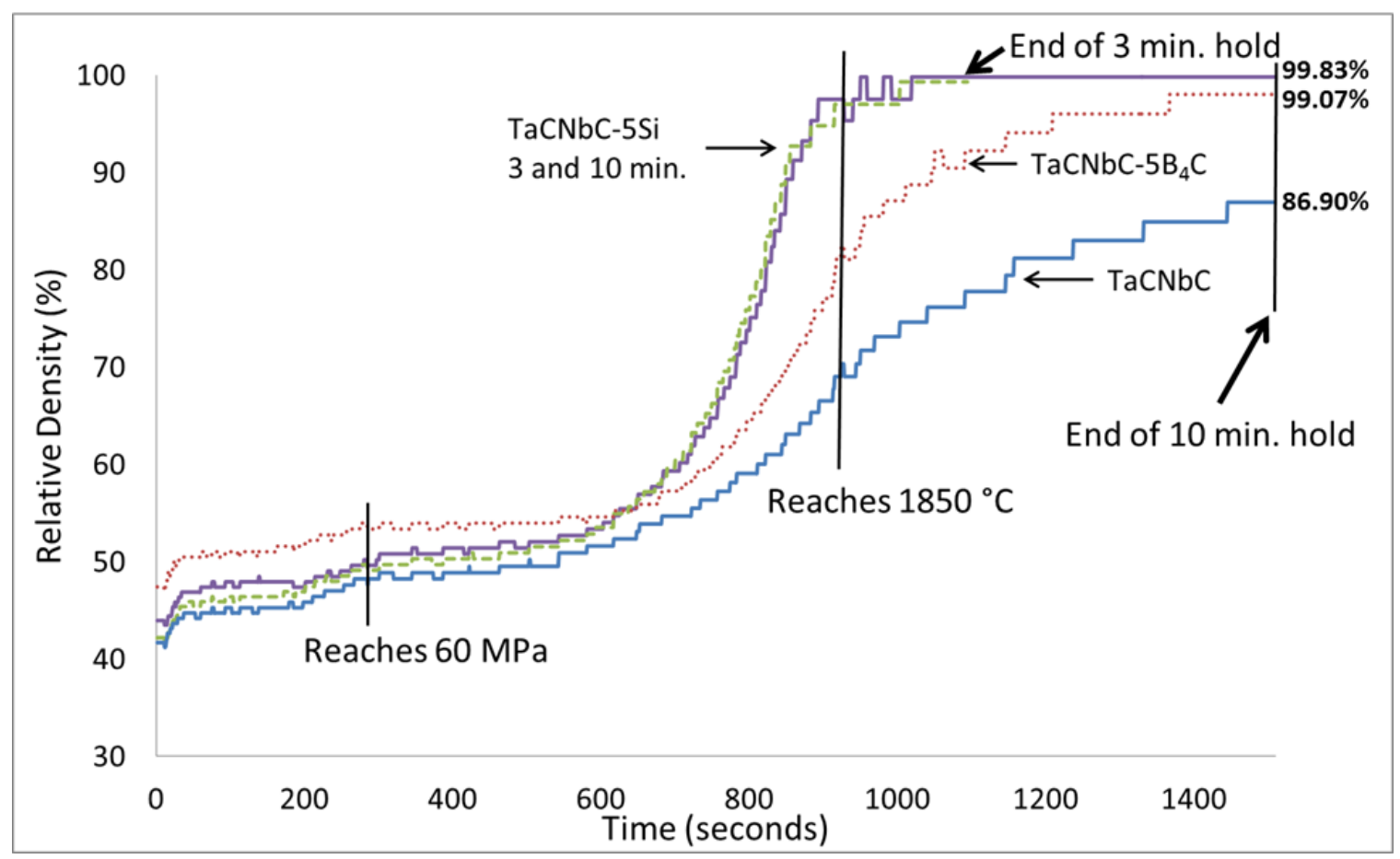

Figure 31: Instantaneous relative densities during the spark plasma sintering process.

Instantaneous relative density is calculated based on the ram displacement data from the SPS process after subtracting out the displacement due to thermal expansion of the dies and the following equation: 


$$
\rho_{\text {instantaneous }}=\left(\frac{L_{\mathrm{f}}}{L}\right) \rho_{\mathrm{f}}
$$

In order to calculate the thickness of the specimen $(L)$ at time $\mathrm{t}$ throughout the SPS process, the initial thickness of the powder is first found by adding the total distance the rams travel throughout the process to the final thickness $\left(L_{\mathrm{f}}\right)$ of the SPS compact measured after sintering. From the initial thickness, the displacement of the ram is subtracted in order to get the instantaneous thickness at time t. The ratio of the final thickness to the instantaneous thickness is then multiplied by the final relative density $\left(\rho_{\mathrm{f}}\right)$ of the sample. This allowed full relative densification to be achieved without the grain growth occurrence that would result from additional dwell time.

\subsection{Microstructural Characterization}

The TaC-NbC sintered compacts with and without sintering additives and GNP were ground using $\mathrm{SiC}$ paper to remove the graphite foil that surrounded the surfaces as a result of SPS processing. The apparent bulk density measurements were carried out using the water immersion technique that employs Archimedes principle as well as using a Helium gas pycnometer (Accupyc 1340, Micromeretics Instrument Corporation, Norcross, GA). The relative densities were calculated as a percentage of true density, which was measured for each starting powder mixture using the Helium gas pycnometer. Phase components of the starting powders along with the sintered samples were analyzed using X-Ray Diffraction (XRD) (Bruker D5000) using $\mathrm{Cu} \mathrm{K \alpha}$ radiation (wavelength of $1.542 \AA$ ) at a scan rate of $1 \%$ minute. The operating voltage and current used were $40 \mathrm{kV}$ and $35 \mathrm{~mA}$, respectively. Fracture surfaces were prepared by cutting off a $5 \mathrm{~mm}$ section of each sintered sample using a low speed diamond saw and then fracturing the cut 
sections using a hammer strike. Scanning electron microscopy (SEM) of the powders and the fracture surfaces of the sintered compacts was carried out using a JEOL JSM-6330 field emission scanning electron microscope (JEOL USA, Inc., Peabody, MA) at an operating voltage of $20 \mathrm{kV}$. The SEM is equipped with energy dispersive X-ray spectroscopy (EDS) used to characterize secondary phase formations as a result of sintering. The surfaces and cross-sections of the remaining portion of samples were ground using diamond grit papers to the $15 \mu \mathrm{m}$ level followed by polishing using diamond suspensions to a $0.25 \mu \mathrm{m}$ finish. A Focused Ion Beam (FIB) (JIB 4500, JEOL USA Inc., Peabody, MA) was used to mill a portion of the polished cross-section with rough milling followed by a precision milling cut in order to reveal the grain sizes and morphologies of the sintered samples.

The bulk GNP samples also used SiC paper to remove the graphite foil layer before being put in the Helium gas pycnometer for density measurement. The sintered compacts were then cut in half using a low speed diamond saw. Half of the compact was fractured and put in the SEM for fracture surface viewing. Both the surface and crosssections of the other half were ground using SiC papers to a 600-grit finish to be used for mechanical property evaluation.

\subsection{Evaluation of Mechanical Properties}

\subsubsection{Vickers Hardness}

Vickers hardness was measured by indenting on the polished cross-sections of the sintered samples. For the samples with GNP addition, indentations were performed on both the cross-section as well as the surface in order to compare the values with respect to 
the aligned orientation of the GNP. Indentations were performed using a LECO LM810AT (LECO Corporation, St. Joseph, MI) microindentation hardness testing system at a load of 1000 grams and a dwell time of 10 seconds. A total of 8 indentations were performed on each sample and the average and standard deviations were calculated.

\subsubsection{In-Situ High Load Instrumented Indentation}

Instrumented indentation was performed using a linear, screw driven micro-load frame, (SEMtester 1000, MTI Instruments, Inc., Albany, NY) shown in Figure 32a. The micro-load frame has a load capacity of $4500 \mathrm{~N}$ with an accuracy of $0.2 \%$ and a linear movement resolution of $20 \mathrm{~nm}$. The load frame is controlled using an interface and MTESTQuattro software (ADMET, Norwood, MA) which also outputs the loaddisplacement data.
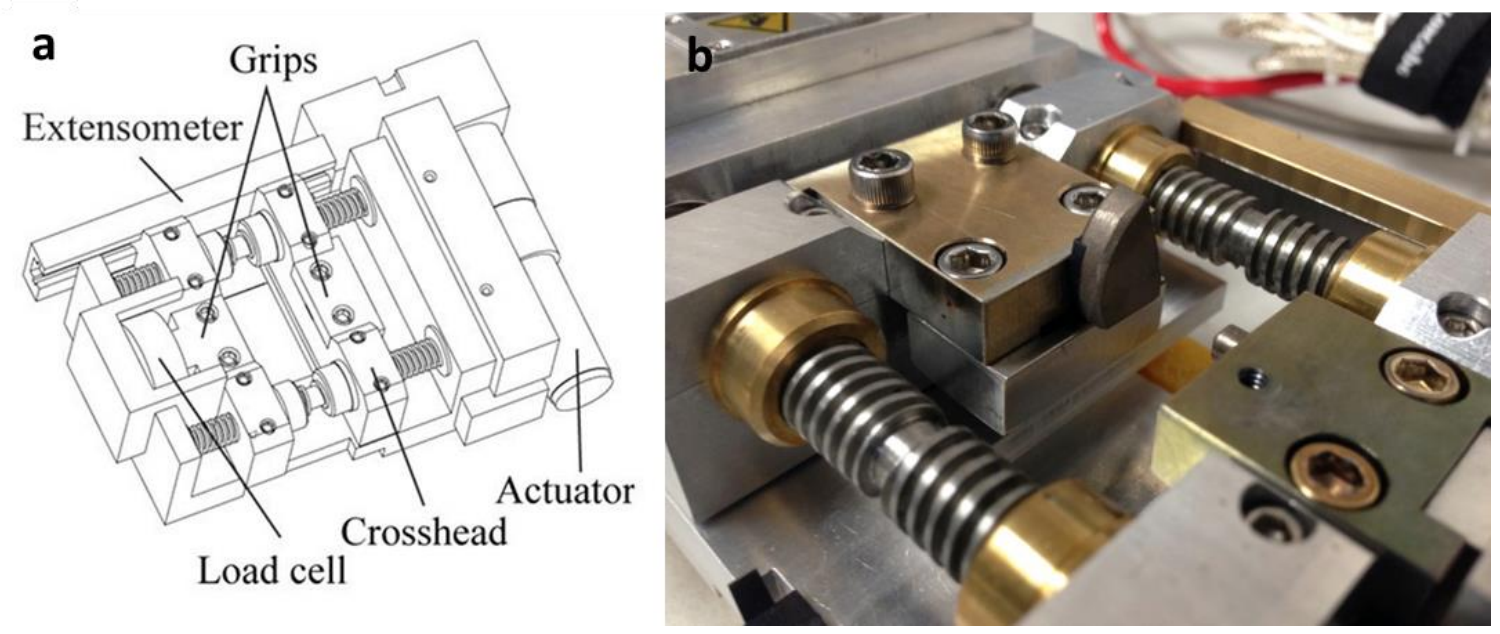

Figure 32: (a) Schematic of the micro-load testing frame used for in-situ instrumented indentation. (b) View of the sample situated across from the indentation tip.

The load frame was instrumented with an approximately $1 \mu \mathrm{m}, 120^{\circ}$ conospherical diamond tip loaded across from the prepared sample (Figure 32b). These depth sensing 
indentation tests are conducted within the chamber of an electron microscope for realtime imaging at the microscopic level (Figure 33).

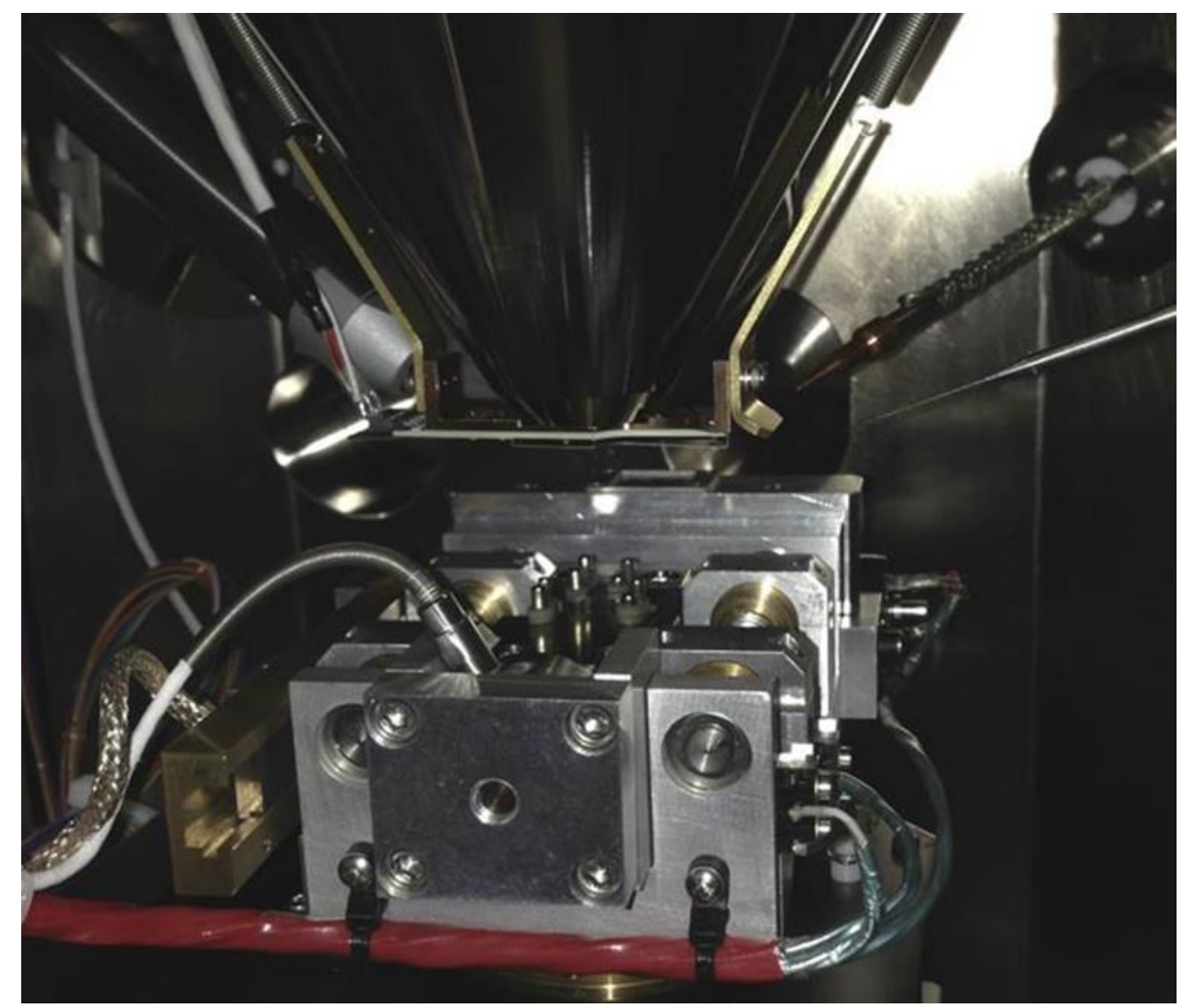

Figure 33: Image of load frame inside the scanning electron microscope for in situ testing.

The combination of the indentation load-displacement measurements with highresolution real-time imaging provides a new perspective on the influence of GNP reinforcement on the ceramic matrix.

For bulk GNP, instrumented indentation was carried out on both the surface (outof-plane GNP orientation) as well as the orthogonal direction (in-plane GNP orientation) as shown in Figure 34. 

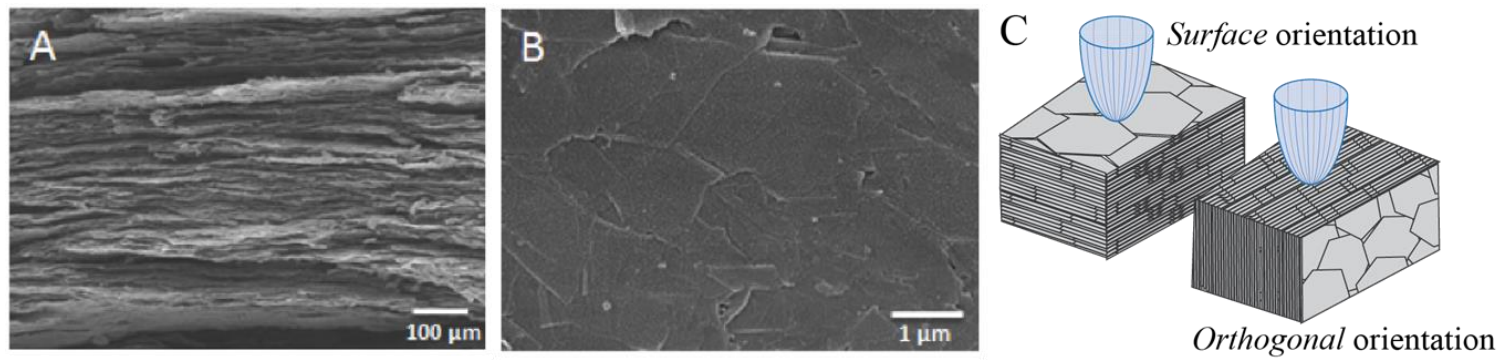

Figure 34: SEM images of (A) the fracture surface and (B) the top surface of spark plasma sintered bulk GNP compacts. (C) Schematic of the surface and orthogonal indentation directions.

Indentation was performed in a vacuum atmosphere using a displacement control of 0.05 $\mathrm{mm} / \mathrm{min}$. to a displacement of $0.5 \mathrm{~mm}$ and then unloaded at the same rate. By studying the intrinsic deformation mechanisms in bulk consolidated GNP with respect to orientation, the anisotropy of the mechanism occurrences and their associated energy dissipation capabilities can be understood.

For the $\mathrm{TaC}-\mathrm{NbC}$ composites with and without sintering additive and GNP addition, the in-situ instrumented indentation tests were performed using the same diamond tip but under load control. A loading function of $100 \mathrm{~N} / \mathrm{min}$. up to $500 \mathrm{~N}$ followed by a dwell time of 5 minutes at $500 \mathrm{~N}$ before being unloaded at $100 \mathrm{~N} / \mathrm{min}$. was used. The resulting load-displacement curves were compared as well as the resulting residual indentation fracture by way of SEM imaging.

\subsubsection{Compression Testing}

Diametral compression testing was performed on the sintered compacts with GNP inclusion to get the tensile strength of each sample. Testing is performed using a MTS electromechanical universal testing system (MTS Criterion Series 43, MTS Systems Corporation, Eden Prairie, MN) by compressing a sintered sample at a rate of 
$0.05 \mathrm{~mm} / \mathrm{min}$. until fracture. The specimen is positioned between two high-strength, hardened steel plates as shown in Figure 35.

\section{Load applied-P}

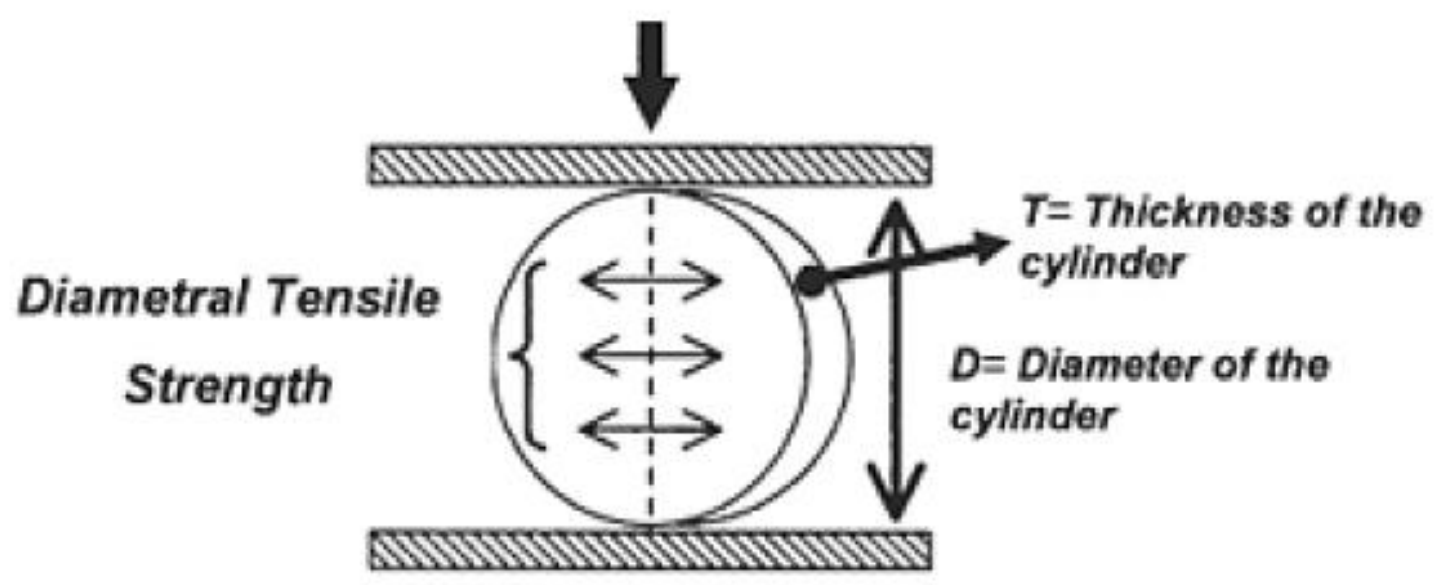

Figure 35: Schematic of the diametral compression test setup.

Sintered samples were ground to a thickness of $3.5 \mathrm{~mm}$ and a final diameter of 19.75 $\mathrm{mm}$. The tensile fracture strength is calculated by equation 5 .

$$
\sigma=\frac{2 P}{\pi D t} \ldots \ldots \ldots \ldots . . . \text { Equation (5) }
$$

Where $\sigma$ is the fracture tensile strength, $\mathrm{P}$ is the load at which fracture occurs, $\mathrm{D}$ is the diameter of the sample, and $\mathrm{t}$ is the sample thickness. This equation results from taking the Hertzian equations for stress-states (Equations (6) through (8)) of elastic disks under point loading and making the following assumptions [67]:

1. The maximum tensile principal stress is responsible for the sample failure and occurs at the center of the disk (therefore $\mathrm{x}=\mathrm{y}=0$ in the Hertzian equations).

2. The strains involved are small. 
3. There is frictionless contact at the boundaries.

$$
\begin{aligned}
& \sigma_{x}=\frac{-2 P}{\pi t}\left(\frac{x^{2}(R-y)}{\beta_{1}^{4}}+\frac{x^{2}(R+y)}{\beta_{2}^{4}}-\frac{1}{2 R}\right) \ldots \ldots \ldots \ldots \ldots \ldots \ldots \ldots . . \text { Equation (6) } \\
& \sigma_{y}=\frac{-2 P}{\pi t}\left(\frac{(R-y)^{3}}{\beta_{1}^{4}}+\frac{(R+y)^{3}}{\beta_{2}^{4}}-\frac{1}{2 R}\right) \ldots \ldots \ldots \ldots \ldots \ldots \ldots \ldots . . \text { Equation (7) }
\end{aligned}
$$

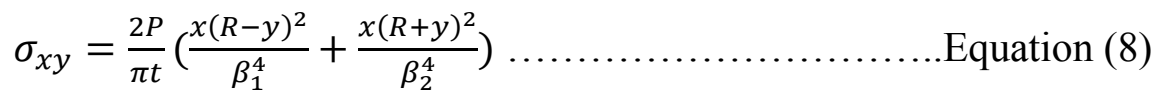

The areas under the load-displacement curves were calculated as a measure of the fracture energy.

\subsection{Oxidation Studies}

Oxidation testing was carried out using a SG 100 DC plasma gun (Praxair, Danbury, CT) that is typically used for high temperature spray deposition of coatings. The gun is operated at a power of $32 \mathrm{~kW}$ and generates plasma with the primary argon gas flowing at $56 \mathrm{slpm}$ and helium as the secondary gas flowing at $60 \mathrm{slpm}$. The high temperature that can be obtained (up to $\sim 10,000^{\circ} \mathrm{C}$ ) and the flow speed usually used to deposit powders $(75-300 \mathrm{~m} / \mathrm{s})$ help to simulate the extreme conditions that the tested materials would be used. As shown in the schematic of the testing setup (Figure 36), a standoff distance of $75 \mathrm{~mm}$ is used between the plasma gun and the sample to ensure that it is exposed to ample oxygen instead of solely being immersed in the plasma flow. A thermocouple is in contact with the back side of the sample and a front side temperature is found using an accuraspray in-flight particle diagnostic sensor (Tecnar Automation Ltd., QC, Canada) to estimate the temperature at a standoff distance of $75 \mathrm{~mm}$. 


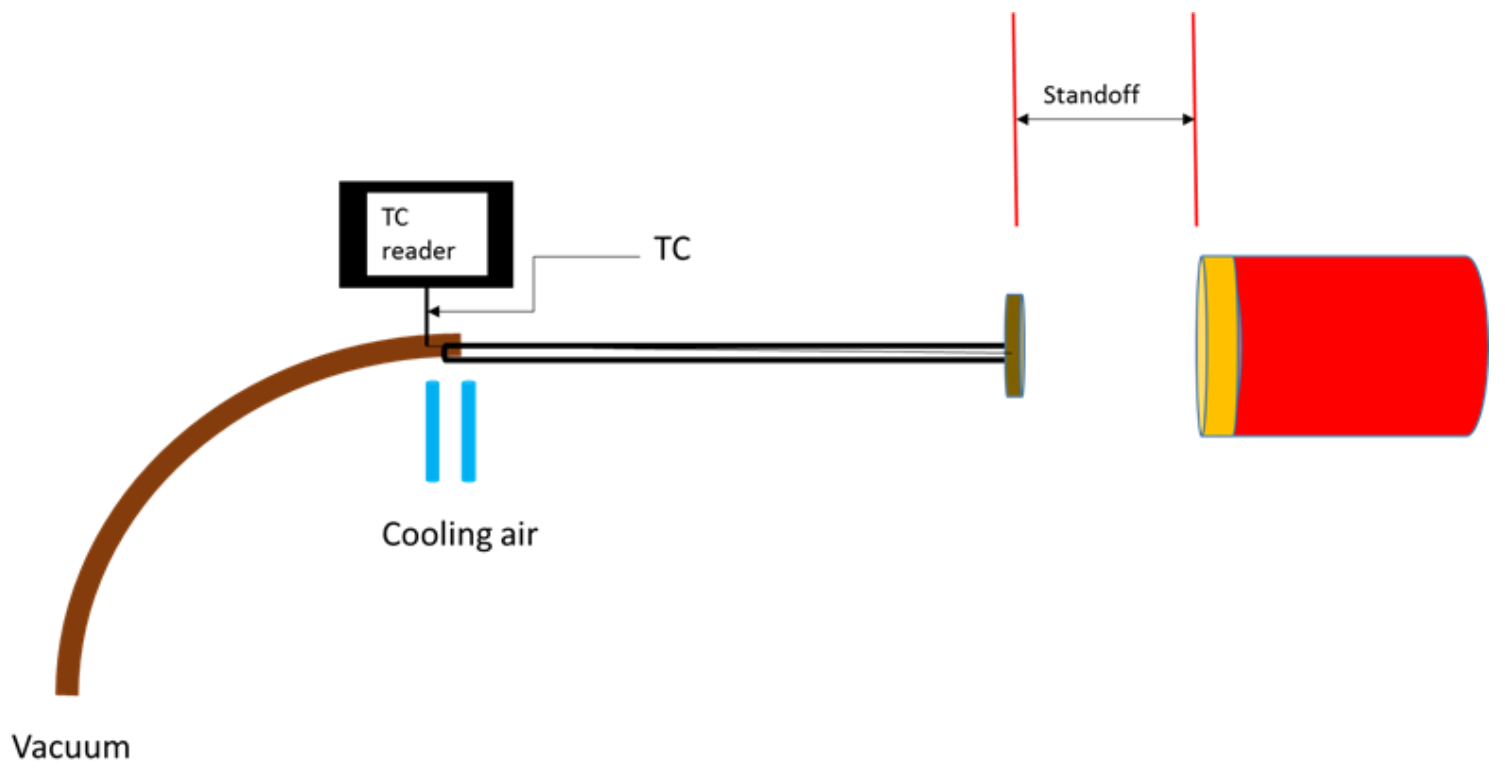

Figure 36: Schematic of the oxidation testing setup. 


\section{Chapter IV: Results and Discussion}

The overall objective of this research is to enhance the potential for GNP reinforced TaC-NbC composites to be used in extreme environmental conditions. In order to further the GNP's potential as a reinforcement, the intrinsic toughening mechanisms must be fully understood. Hypotheses for increased toughness in composites reinforced with GNP can be attributed to three different regimes; (i) property changes during processing, (ii) increased load capacity prior to initial crack propagations, and (iii) crack propagation suppression mechanisms. In order to achieve our overall objective, three specific objectives must be met which will investigate the hypotheses for increased toughness with GNP addition. First, the toughening mechanisms of GNP need to be investigated in terms of their energy dissipation potential. Due to the anisotropy of the GNP deformation mechanisms, a study is first done on bulk GNP consolidated by SPS which aligns the GNP in a uniform orientation allowing us to determine the deformation mechanisms that are most prevalent in each direction. This investigation will shed light on the regime (ii) toughening mechanisms including bending, kinking, and sliding which dissipate energy that would otherwise lead to crack propagation in a composite. The second objective is to achieve as close to full relative density as possible with a uniform, refined microstructure of the $\mathrm{TaC}-\mathrm{NbC}$ in order to improve the reliability of the ceramic compacts. To achieve this, the role of SPS processing parameters as well as the use of sintering additives to enhance densification will be explored. For the third objective, what has been learned from the first two objectives will be combined to synthesize fully dense, GNP reinforced TaC-NbC with improved toughening. This objective will shed light on 
all three regimes hypothesized to increase toughening as a result of GNP reinforcement in composites.

4.1 Intrinsic Deformation Mechanisms of GNP and Their Energy Dissipation Capabilities with Respect to Orientation

In order to achieve the highest toughening in $\mathrm{GNP}$ reinforced $\mathrm{TaC}-\mathrm{NbC}$, the intrinsic deformation mechanisms of the GNP need to be realized in terms of their energy dissipation capabilities. Bulk GNP consolidated by SPS results in GNP aligned in an orientation normal to the pressing axis of the SPS machine. This alignment enables investigation of the GNP intrinsic deformation mechanisms as a function of the GNP anisotropy. The motive behind this investigation is to gain a fundamental understanding of the effect of GNP orientation on toughening through the combination of depth sensing indentation and in situ imaging using an electron microscope. Using the combined experimentation and high magnification imaging, an understanding of which mechanisms occur in each orientation and the associated work of indentation that is required to initiate and propagate each mechanism can be determined. Information related to work and energy can be used to predict both bulk GNP response, as well as improve the mechanistic understanding of GNP reinforced composites.

\subsubsection{Sample Synthesis}

Bulk graphene nanoplatelets (xGNP-M-5, XG Sciences, Lansing, MI) were consolidated by spark plasma sintering at $1850^{\circ} \mathrm{C}$ with a heating rate of $200^{\circ} \mathrm{C} / \mathrm{min}$., a dwell time of 10 minutes, and a pressure of $80 \mathrm{MPa}$. The consolidated specimen was a 3 mm thick disk with a diameter of $20 \mathrm{~mm}$. Density measurements carried out using a 
Helium pycnometer revealed a density of $2.01 \mathrm{~g} / \mathrm{cm}^{3}$ which is higher than the measured pre-sintered GNP particles of $1.82 \mathrm{~g} / \mathrm{cm}^{3}$. This increase is attributed to the consolidation of the particles.

Figure 37a shows an SEM image of the fracture surface of the SPS consolidated bulk GNP. As can be seen, the GNP are aligned in an orientation normal to the pressing axis of the SPS machine. Figure 37b shows an SEM image of the top surface of the consolidated pellet which shows that the GNP are layered but still retain their platelet shape. The consolidation of overlapping GNP at the edges tends to form a grain boundary-like structure as shown in Figure 37b.
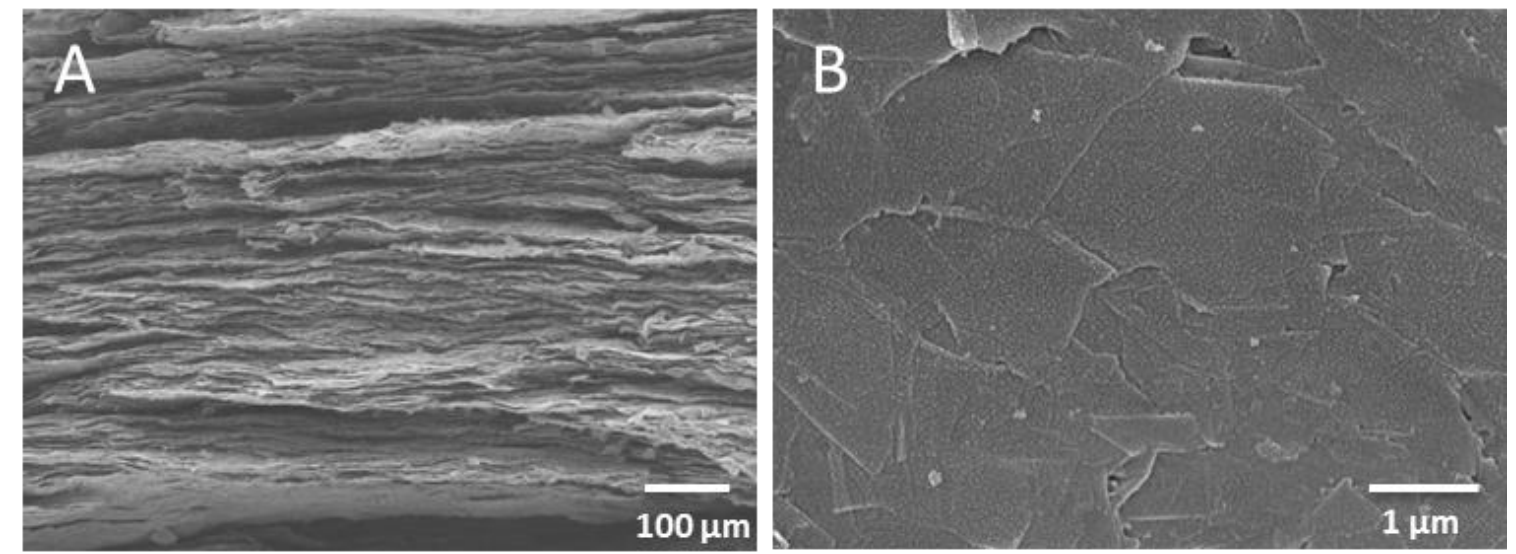

Figure 37: SEM images of A) fracture surface and B) top surface of spark plasma sintered bulk GNP pellet.

\subsubsection{In-Situ Indentation}

In situ indentation was carried out inside a dual beam Focused Ion Beam/SEM. A linear, screw driven micro-load frame instrumented with an approximately $1 \mu \mathrm{m}, 120^{\circ}$ conospherical diamond tip loaded across from the prepared sample was used for the indentation experiments. The consolidated bulk GNP sample was prepared by cutting the 
$20 \mathrm{~mm}$ diameter, $3 \mathrm{~mm}$ thick cylindrical sample in half and polishing the surface and cross section to a metallographic finish with a surface roughness of $0.25 \mu \mathrm{m}$. Figure 38 illustrates the two loading directions identified as surface and orthogonal.

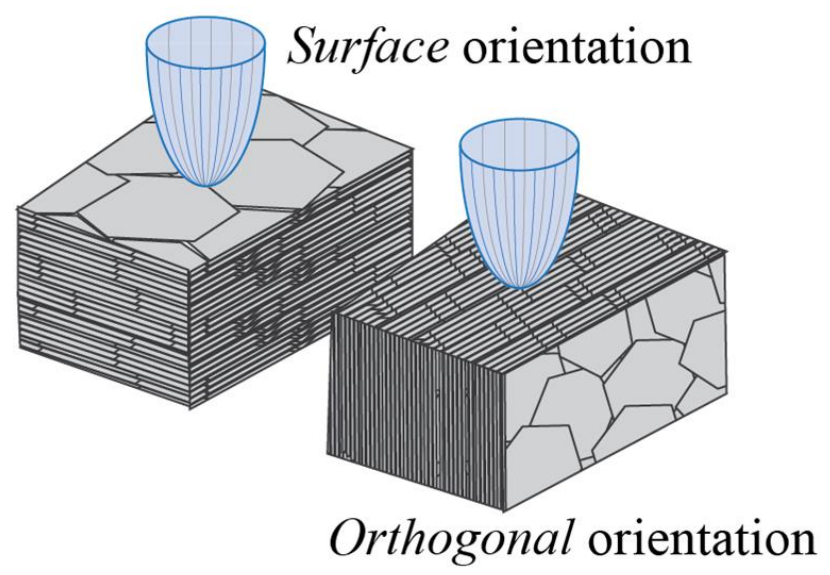

Figure 38: Schematic showing the surface and orthogonal indentation orientations.

Indentation was carried out under vacuum atmosphere at a displacement controlled rate of $0.05 \mathrm{~mm} / \mathrm{min}$. to $0.5 \mathrm{~mm}$ and then unloaded at the same rate. Real time video of the indentations as well as the load-displacement curve outputs enable characterization of deformation mechanisms and corresponding energy dissipation.

From the indentation load-displacement curves (Figure 39 and Figure 40), it is evident that the orientation of the GNP with respect to the loading direction has a dramatic effect on the mechanical properties. Indentation of the specimen in the orthogonal direction required a maximum load of $42.89 \mathrm{~N}$ (Figure 40) to reach a displacement of $0.48 \mathrm{~mm}$ whereas indentation performed on the surface required a maximum load of only $20.40 \mathrm{~N}$ (Figure 39) in order to reach a displacement of $0.46 \mathrm{~mm}$. Loading was carried out in displacement control and started with the indenter tip slightly offset from the sample which is the reason the final displacements differ by $0.02 \mathrm{~mm}$. 
Loading in the orthogonal direction (in-plane GNP orientation) is expected to be much higher since the high young's modulus of GNP (1 TPa) [66] is reported for this direction.
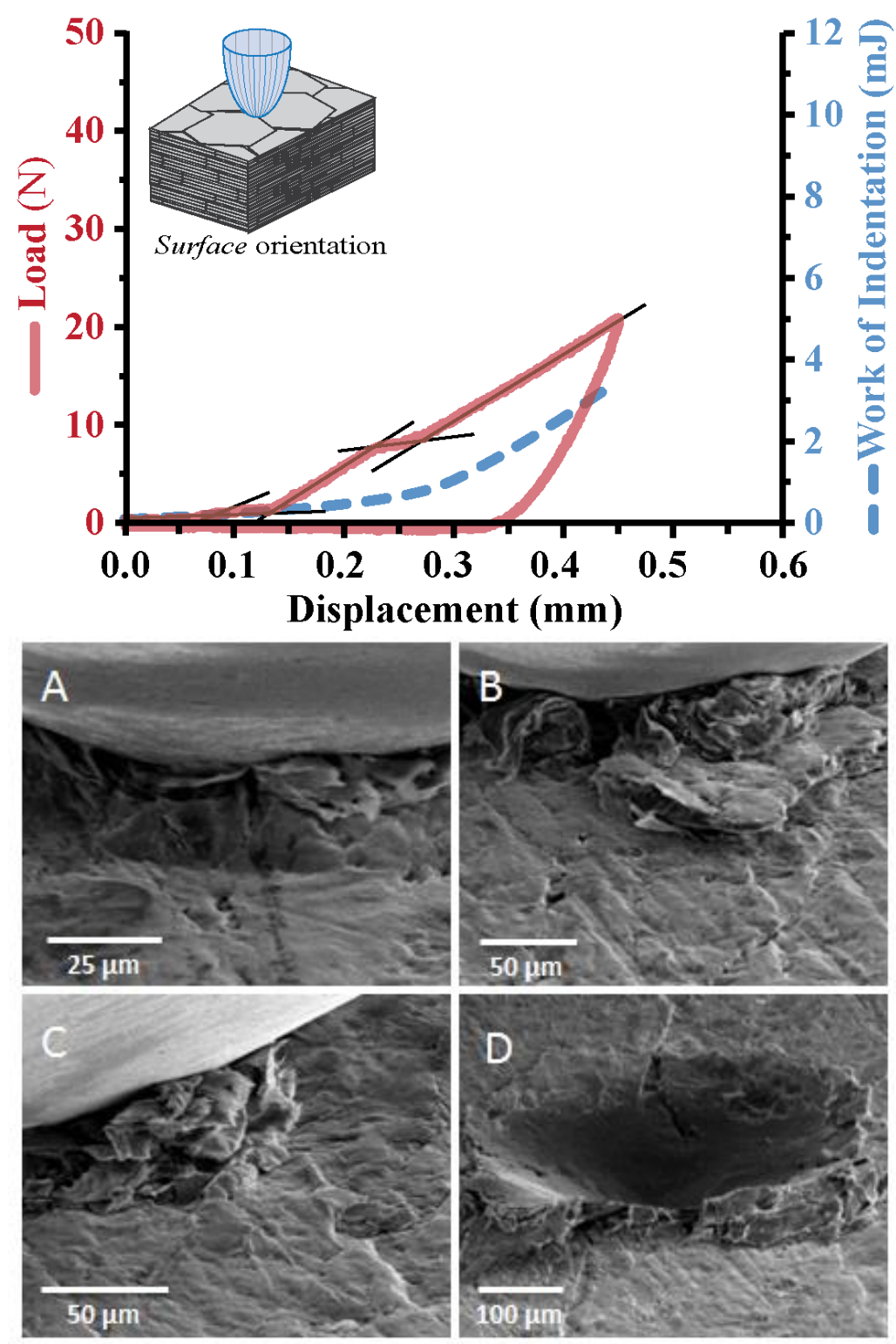

Figure 39: Surface indent Load-Displacement and Work-Displacement curves and images of deformation occurrences. 

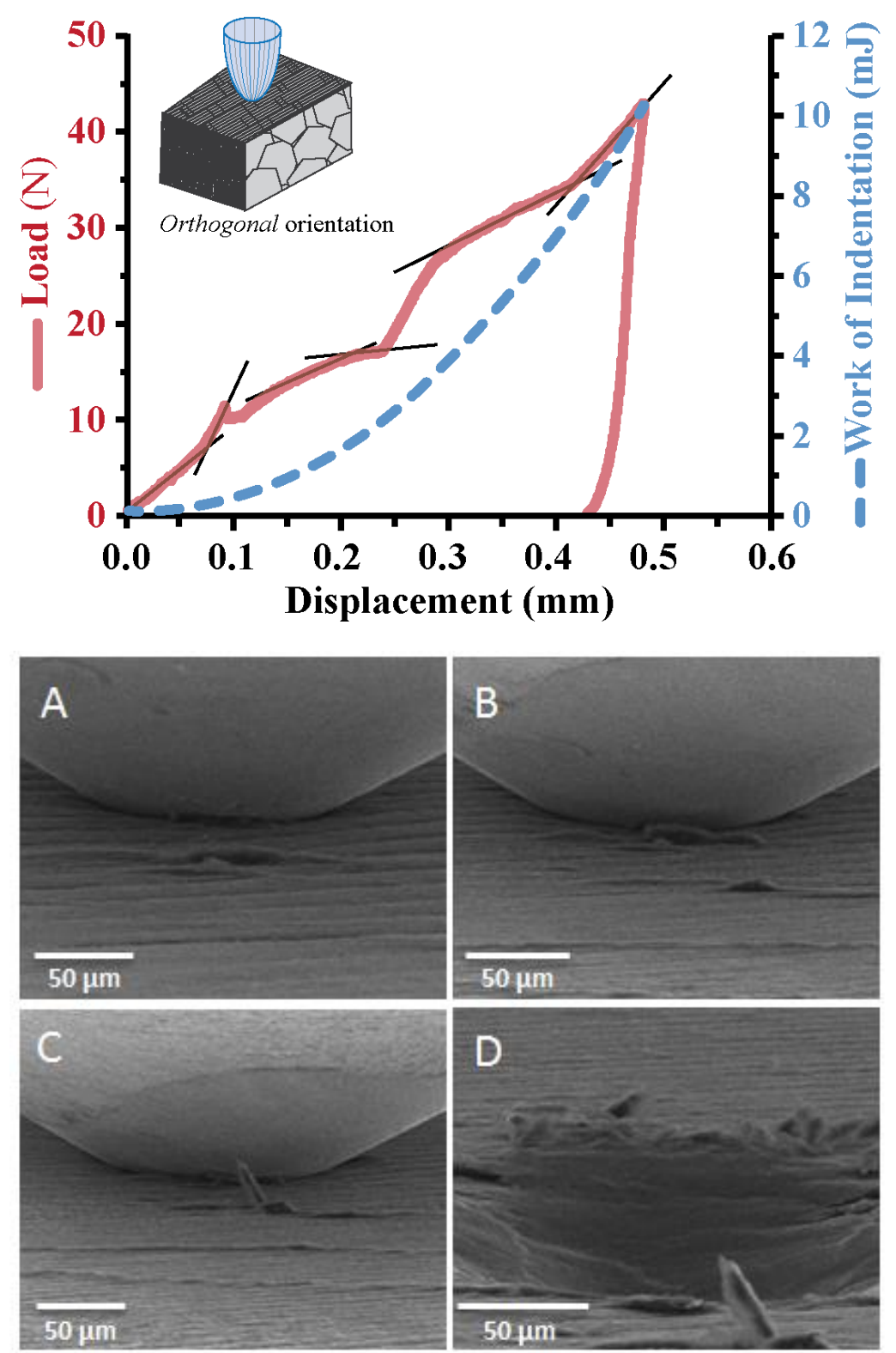

Figure 40: Orthogonal indent Load-Displacement and Work-Displacement curves and images of deformation occurrences.

The work of indentation versus displacement curves were calculated from the area under the loading curves. It is seen that the work required to achieve the same displacement is much higher when indentation occurs orthogonal to the GNP surface. The total work of indentation in the orthogonal direction was $10.02 \mathrm{~mJ}$ or about $270 \%$ more than the total work of indentation on the surface which was $3.71 \mathrm{~mJ}$. 
When looking at the Load-Displacement data from the surface indent (Figure 39) there are five distinct changes in the slope. This change in slope can be correlated to the deformation mechanisms at that stage and amount of normalized associated energy being dissipated in the sample. Normalized energy is found by taking the slope at the various listed displacement ranges and extrapolating that slope over a displacement range from 0 $\mathrm{mm}$ to $0.45 \mathrm{~mm}$. This normalized energy is the work of indentation energy that would result if the slope stayed constant over the entire displacement range of $0.45 \mathrm{~mm}$ and allows for a comparison to be made between the dissipating energies with what deformation mechanisms are occurring.

Table 6 lists the displacement range (indentation depth), the time ranges on the indentation video, and the normalized associated energy for each distinct slope regime in the load-displacement curve of the surface indent.

Table 6: Surface indent energy dissipation results.

\begin{tabular}{|c|c|c|c|}
\hline Slope & $\begin{array}{c}\text { Displacement Range } \\
(\mathbf{m m})\end{array}$ & $\begin{array}{c}\text { Time Range in } \\
\text { Video (min.) }\end{array}$ & $\begin{array}{c}\text { Normalized Associated } \\
\text { Energy (mJ) }\end{array}$ \\
\hline 1 & $0.010-0.075$ & $0: 12-0: 51$ & 1.30 \\
\hline 2 & $0.083-0.100$ & $0: 56-1: 06$ & 0.01 \\
\hline 3 & $0.108-0.200$ & $1: 12-2: 06$ & 5.96 \\
\hline 4 & $0.216-0.233$ & $2: 16-2: 27$ & 6.06 \\
\hline 5 & $0.258-0.455$ & $2: 42-4: 39$ & 1.85 \\
\hline
\end{tabular}

During slope regime 1, a small amount of sheet delamination occurred in the form of GNP sliding followed by bending over of the pileup of delaminated GNP (Figure 39A). The energy dissipation associated with this regime is small because the indentation is 
mainly occurring on GNP aligned in an out-of-plane orientation. The mechanisms associated with this small energy are the sliding and bending of the GNP, which do not dissipate much energy due to the weak van der Waals forces between GNP layers. This sliding mechanism is evident in the post-indentation SEM image Figure 41B where it can be seen that the GNP are separating at the indent site and sliding in a direction perpendicular to the indentation load. The wrinkling in the GNP that is seen on the right hand side of the figure (marked with arrows) is further evidence of the lateral GNP movement by the sliding mechanism. In the displacement range corresponding to slope 2 we saw the pileup from regime 1 easily folding over with very little new sheet delamination. At the end of this regime, a large area of slide-out occurs as shown in Figure 39B. The results of this regime exemplify what was expected, that the GNP sliding mechanism dissipates the least amount of energy. During the slope 3 regime there is significant new sheet delamination in the form of bending and kinking occurring all around the indentation tip. The large increase in the associated energy occurs as a result of the onset of GNP kinking. A change of orientation is observed from out-of-plane to inplane GNP alignment to give a mixed-plane loading. These bending and kinking mechanisms occur in the areas surrounding the indenter tip causing pileup, shown in Figure 41C. The mechanisms that are observed in regime 4 are very similar to what was seen in regime 1. The areas of kinking and pileup are pushed away from the indentation tip by sheet delamination and slide-out as seen in Figure 39C. Therefore the benefits of the kinked GNP are no longer in contact with the indenter and the associated dissipation energy is again the result of the weak van der Waals forces between sliding GNP layers. Another mechanism that occurred as a result of the sheet sliding is GNP pull-out (Figure 
41D) which occurred when the overlapping GNP that were connected at the grain boundaries separate causing a crack. In regime 5, similar deformation mechanisms as regime 3 , bending and kinking, are occurring. This again creates the mixed-plane loading that dissipates a greater energy.
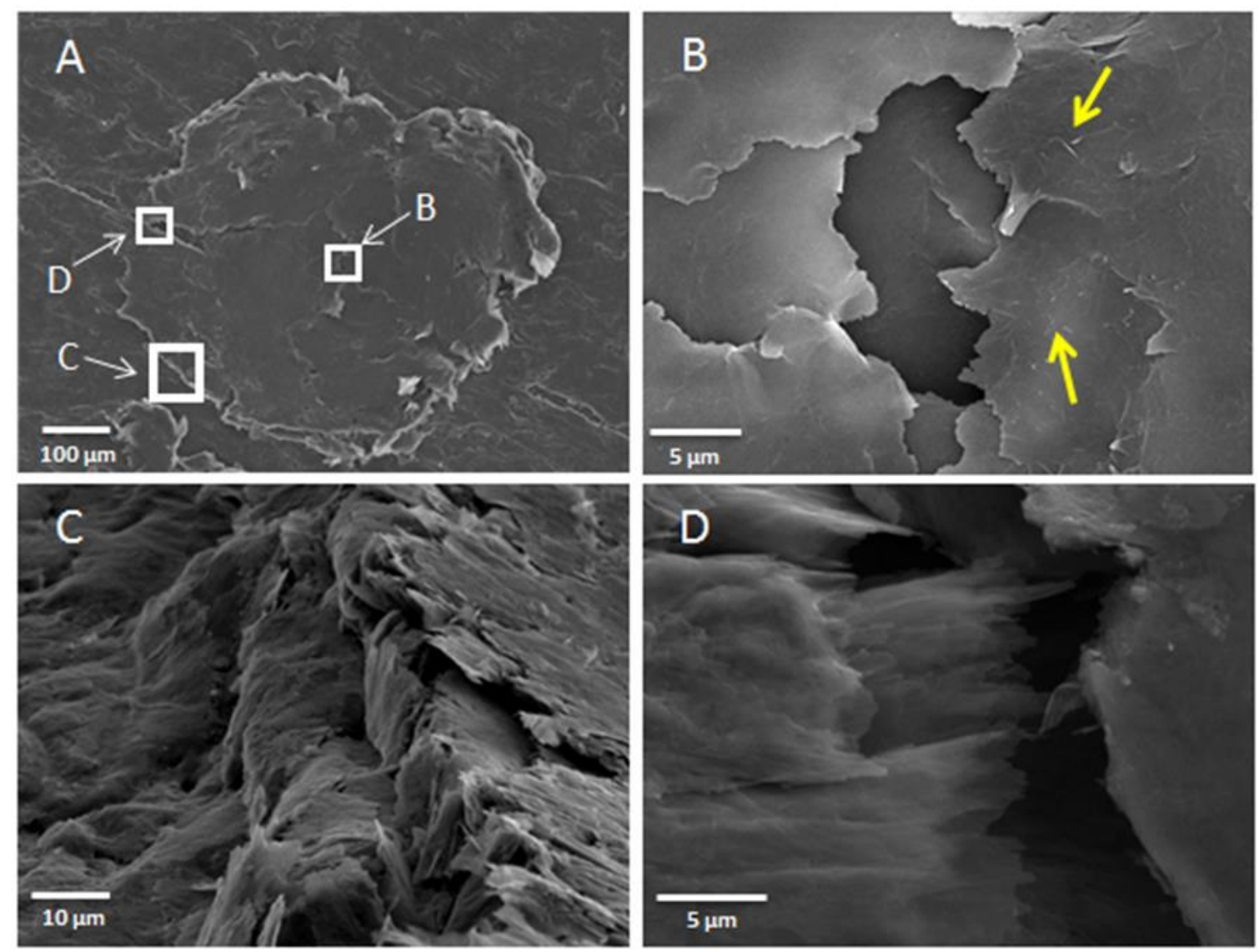

Figure 41: SEM images of A) overall residual indentation and magnified images of deformation mechanisms corresponding to the locations marked in image (A) as a result of the surface indentation: B) sheet sliding with arrows marking wrinkling, C) bending and kinking, and D) sheet pull-out.

Table 7 lists the displacement range, the time ranges on the indentation video, and the normalized associated energy for each distinct slope regime in the load-displacement curve of the indent in the orthogonal direction. 
Table 7: Orthogonal indent energy dissipation results.

\begin{tabular}{|c|c|c|c|}
\hline Slope & $\begin{array}{c}\text { Displacement } \\
\text { Range }(\mathbf{m m})\end{array}$ & $\begin{array}{c}\text { Time Range in Video } \\
(\mathbf{m i n} .)\end{array}$ & $\begin{array}{c}\text { Normalized Associated } \\
\text { Energy }(\mathbf{m J})\end{array}$ \\
\hline 1 & $0.000-0.060$ & $0: 03-0: 38$ & 9.08 \\
\hline 2 & $0.080-0.090$ & $0: 50-0: 56$ & 23.49 \\
\hline 3 & $0.120-0.230$ & $1: 13-2: 20$ & 4.92 \\
\hline 4 & $0.231-0.280$ & $2: 21-2: 40$ & 17.48 \\
\hline 5 & $0.320-0.400$ & $3: 14-4: 02$ & 5.75 \\
\hline 6 & $0.420-0.480$ & $4: 14-4: 49$ & 12.62 \\
\hline
\end{tabular}

During the displacement range of regime 1, the indenter begins compressing the GNP oriented in the in-plane direction and bending starts to occur around the indenter tip (Figure 42D). In the displacement range corresponding to regime 2, compression occurs perpendicular or outwards from the indenter tip. This is evident by what appears to be buckling, or GNP push-out, that occur because the outward pressure begins compressing GNP away from the indenter tip. At the end of this regime at a displacement of about $0.09 \mathrm{~mm}$, a large push-out occurs (Figure 40A) that relieves the pressure that is reinforcing the GNP at the indent site to remain in the high energy dissipating in-plane direction. This push-out deformation coincides with the drop in load seen in Figure 40 . This is where regime 3 begins. After the compression pressure is relieved, the normalized energy dissipation returns to a value closer to what was seen in regime 1 and more bending occurs around the indenter tip. As the displacement continues deeper, smaller, more frequent push-out formations are seen further out from the indenter tip. This again reduces the compression reinforcing the indenter tip location, which is evident in the lower normalized associated energy in this regime. Another possible occurrence is that 
when the compression is relieved, the GNP that were being held in the in-plane orientation by the compressive pressure are now able to bend to give a mixed-plane loading of both in-plane and out-of-plane GNP orientations. At the end of regime 3 and start of regime 4, a large GNP pop-out occurs (Figure 40C) which is evidence that the outward compression is again occurring. This gives larger reinforcement to in-plane alignment of the GNP at the indent site that again requires increased work of indentation. In regimes 5 and 6 , there is no visible evidence to link the energy dissipation values to any specific GNP deformation mechanisms. A possible reason for this is that the deformation mechanisms occur on the back side of the indenter tip. 

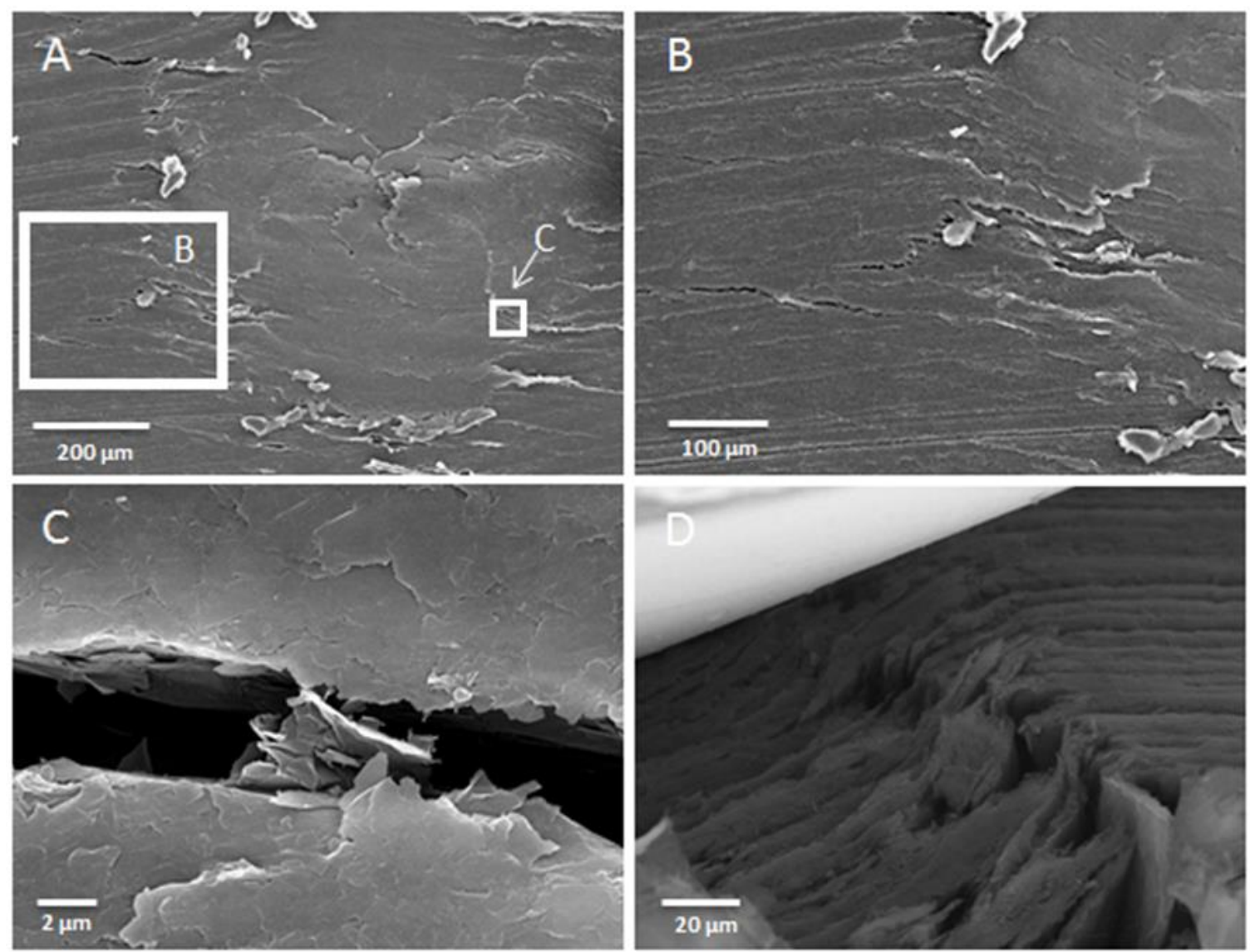

Figure 42: SEM images of A) overall residual indentation and magnified images of deformation mechanisms corresponding to the locations marked in image (A) as a result of orthogonal indentation: B) separation and cracking, C) crack propagation, and D) bending during indentation.

Figure 41A and Figure 42A show SEM images of the residual damage left after indenting on the surface and orthogonal directions, respectively. Indentation performed on the surface resulted in greater pileup. This is expected because of the slide-out that occurred outward from the indentation site. The indentation performed in the orthogonal direction showed almost no pileup. It is evident from Figure 42D that the GNP in contact with the indentation tip experienced bending that reconfigured the GNP alignment to the out-of-plane orientation. In contrast to the cracking that occurred as a result of GNP sheet separation during the surface indent, the cracks that formed during the orthogonal indent 
propagated outward from the bending locations around the tip as shown in Figure 42B and Figure 42C. This occurrence follows what was previously seen by Zhang et al. [19] in a graphene platelet agglomeration of graphene platelet-HA composites under a bending load.

Based on the observed deformation mechanisms and the quantified normalized energy associated with each slope regime, it is possible to arrange the GNP deformation mechanisms in order of energy dissipation capabilities (Table 8).

Table 8: GNP deformation mechanisms in order of energy dissipation capabilities.

\begin{tabular}{|l|l|l|}
\hline Deformation Mechanism & Associated Indent/Regime & $\begin{array}{c}\text { Normalized Associated } \\
\text { Energy of Regime (mJ) }\end{array}$ \\
\hline Reinforcing Compression & Orthogonal (2), (4) & $23.49,17.48$ \\
\hline MLG Push-out, Pop-out & Orthogonal (2), (3), (4) & $23.49,4.92,17.48$ \\
\hline Kinking & Surface (3), (5) & $5.96,6.06$ \\
\hline Bending & Orthogonal (1), (3) & $9.08,4.92$ \\
\hline MLG Pull-out & Surface (3), (5) & $5.96,6.06$ \\
\hline Sliding & Surface (4) & 1.85 \\
\hline
\end{tabular}

When indentation is performed on the surface, the deformation mechanism that dissipates the greatest energy is kinking of the GNP. This is followed by bending of the GNP, GNP pull-out, and sliding. When indentation is performed in the orthogonal direction, the greatest energy is dissipated when compression occurs outward from the indentation site which in turn reinforces the in-plane alignment of the GNP that are in contact with the indenter tip. This is followed by GNP pop-out, push-out, and bending. 
4.1.3 Conclusions Based on the Study of Bulk GNP Deformation Mechanism Anisotropy

The toughening benefits of GNP as a result of deformation mechanisms are highly orientation driven. When indentation is performed on the surface, the energy dissipating deformation mechanism that most readily occurs is GNP sliding followed by bending, kinking, and GNP pull-out. When indentation is performed in the orthogonal direction, the total energy that is dissipated is up to $270 \%$ greater than when indentation is performed on the surface. This is attributed to the fact that the deformation mechanism that occurs most readily during the surface indent, GNP sliding, is also recognized as providing the least amount of energy dissipation due to the weak van der Waals forces. The deformation mechanisms that are observed during the orthogonal indentation are bending, push-out, and GNP pop-out as a result of outward compression. The outward compression can also act to increase the van der Waals forces between layers by decreasing separation between them. This can also increase the friction forces between layers causing a greater energy dissipation leading up to the push-out of the GNPs. The understanding of the fundamental deformation mechanisms and anisotropy of bulk graphene nanoplatelets in this study will enable the engineering of GNP reinforced composites that can be tailored to desired toughening properties.

\subsection{Powder Processing and Sintering Parameters for the Ceramic Composites}

Before consolidating the ceramic composites by spark plasma sintering, analysis was performed to determine the best methods of pre-sintering powder processing and composite compositions. Once the pre-sintered powder processing was determined, analysis was performed to determine the role of the SPS processing parameters on the 
microstructure and mechanical properties of the sintered compacts. The goal of performing this analysis and initial experimentation is to find the parameters that achieve samples with high uniformity and densification in order to improve the reliability of the composites. In this section, preliminary results will be discussed including powder preparation methods, volume of sintering aid addition, and SPS processing parameters and how these initial findings led to the final processing parameters.

\subsubsection{Powder Preparation Methods}

The powder preparation methods initially explored included bath sonication, ball milling, blending, and tip sonication as outlined in section 3.2.1. The goal in experimenting with various preparation methods was to determine how each one affected the dispersion of the additives (both sintering aid and GNP) as well as the resulting uniformity of the powder (frequency and size of agglomerations). For each method described in this section, an arbitrarily chosen composition of $\mathrm{TaC}-\mathrm{NbC}$ with 7 vol.\% $\mathrm{Si}$ addition and 3 vol.\% GNP addition was used.

Bath sonication was the first method explored due to its ease of use. The powder was split into four separate beakers in order to increase the chances of breaking agglomerations. Figure 43a shows that the results of bath sonication included adequate dispersion of the reinforcement phase, however agglomerates of between 10-30 $\mu \mathrm{m}$ were observed for the TaC-NbC powder as well as $30 \mu \mathrm{m}$ agglomerates of GNP. While the bath sonication method has the benefit of being very simple, the resulting agglomerations would impede the uniformity, and thereby the reliability, of the sintered sample. 
High energy ball milling was explored to see if the mechanical mixing would also result in the loosening of agglomeration. While the dispersion of both sintering additive and GNP appeared to be uniform, agglomerates of TaC-NbC powder of up to $100 \mu \mathrm{m}$ were readily observed, as shown in Figure $43 \mathrm{~b}$.

Blending using a standard Black and Decker 5-speed blender was explored to see if the energy imparted to the solution by the blade was enough to break of the agglomerations. Blending resulted in non-uniform dispersion of the $\mathrm{Si}$ additive as well as TaC-NbC and GNP agglomerates up to $60 \mu \mathrm{m}$ and $35 \mu \mathrm{m}$, respectively (Figure 43c).
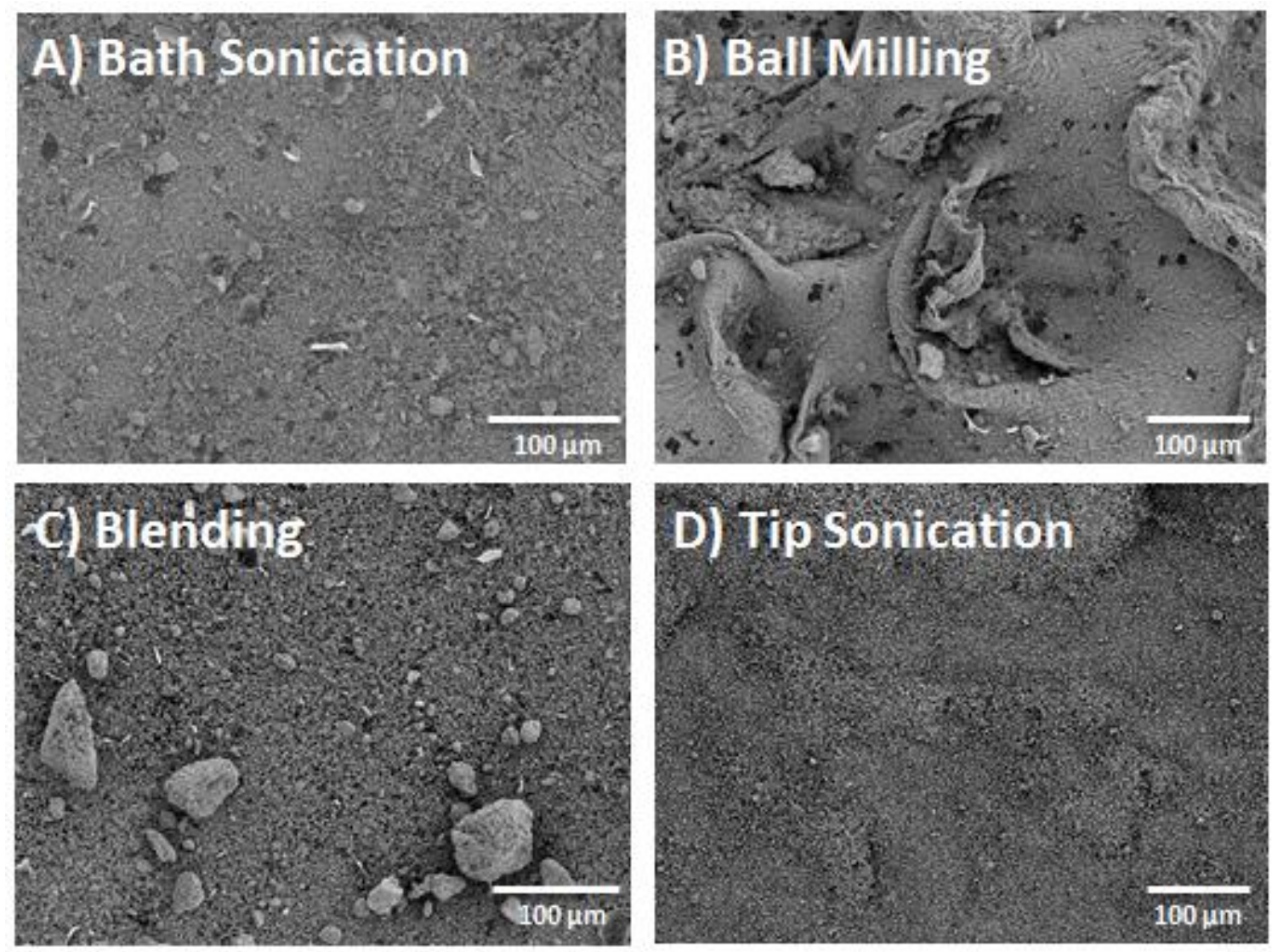

Figure 43: SEM images of TaC-NbC-7 vol.\% Si-3 vol.\% GNP dispersed by a) bath sonication, b) ball milling, c) blending, and d) tip sonication. 
The dispersion method that was ultimately used was the tip sonication method due to the uniform dispersion and the ability of the high energy imparted to the system to break up the agglomerations. Figure $43 \mathrm{~d}$ shows the greatest powder homogeneity compared to the other methods and only sporadic, loose agglomerations up to $10 \mu \mathrm{m}$ could be seen. All samples from here on out were prepared using tip sonication.

\subsubsection{Amount of Sintering Aid Addition}

Initial experimentation was performed using both 5 vol. $\%$ and $10 \mathrm{vol} . \%$ of added sintering aid for enhanced densification. The resulting density and mechanical properties were compared to determine the ideal amount of sintering additive.

The desired densification (>99\%) was achieved with the addition of 5 vol.\% sintering aid addition and did not benefit noticeably by increasing up to 10 vol.\% addition as shown in Figure 44. The elastic modulus and hardness values (Figure 45 and Figure 46 , respectively) also did not benefit noticeably when increasing from 5 to $10 \mathrm{vol} \% \mathrm{~B}_{4} \mathrm{C}$ addition and decreased when increasing from 5 to 10 vol.\% Si addition. Based on these initial findings, 5 vol.\% sintering additive was chosen for use in all subsequent samples with sintering aid. By using the minimal necessary amount of sintering aid, we expect to retain more of the high temperature properties that make $\mathrm{TaC}-\mathrm{NbC}$ a possible UHTC for extreme environment applications. 


\section{Relative Density}

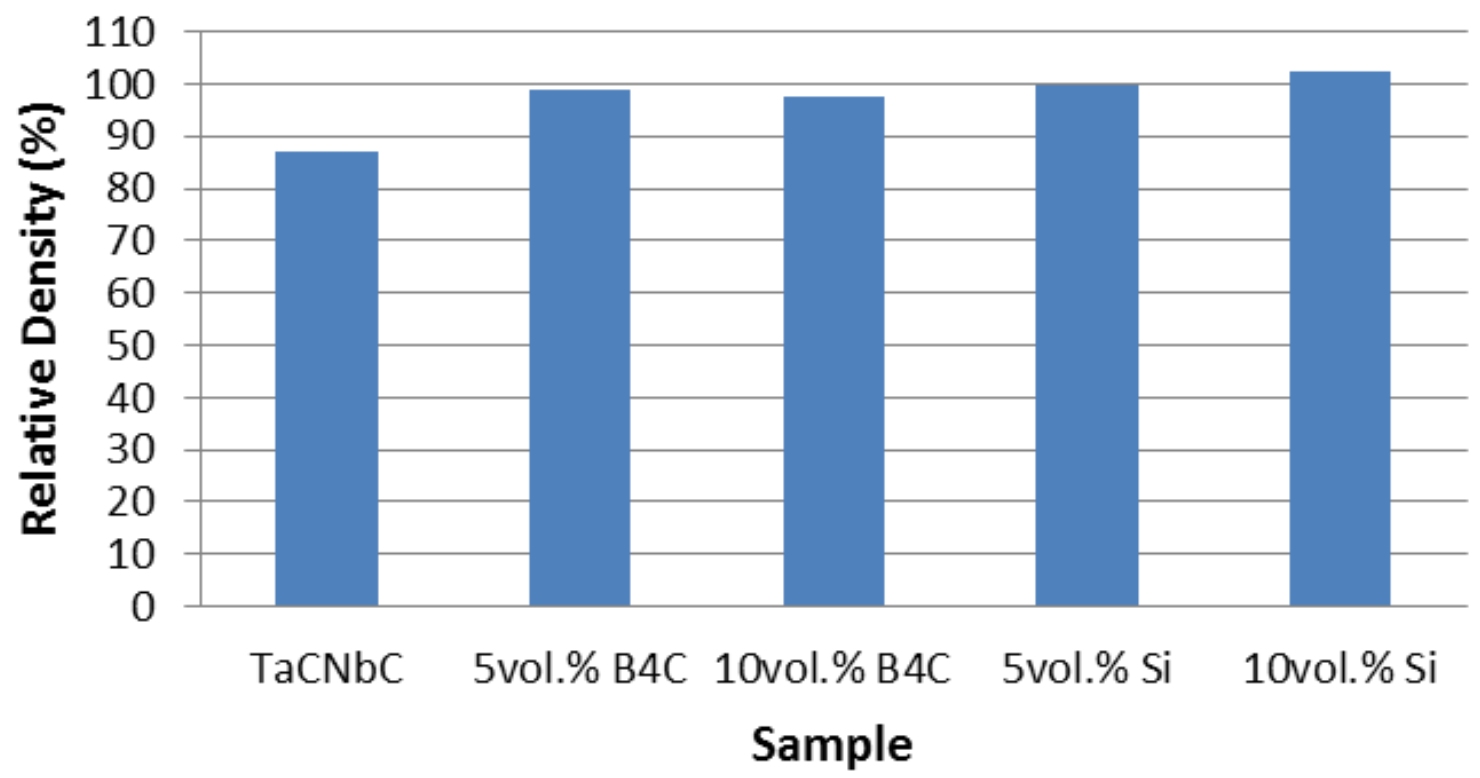

Figure 44: Relative density measurements using 5 and 10 vol.\% sintering aid addition.

Elastic Modulus

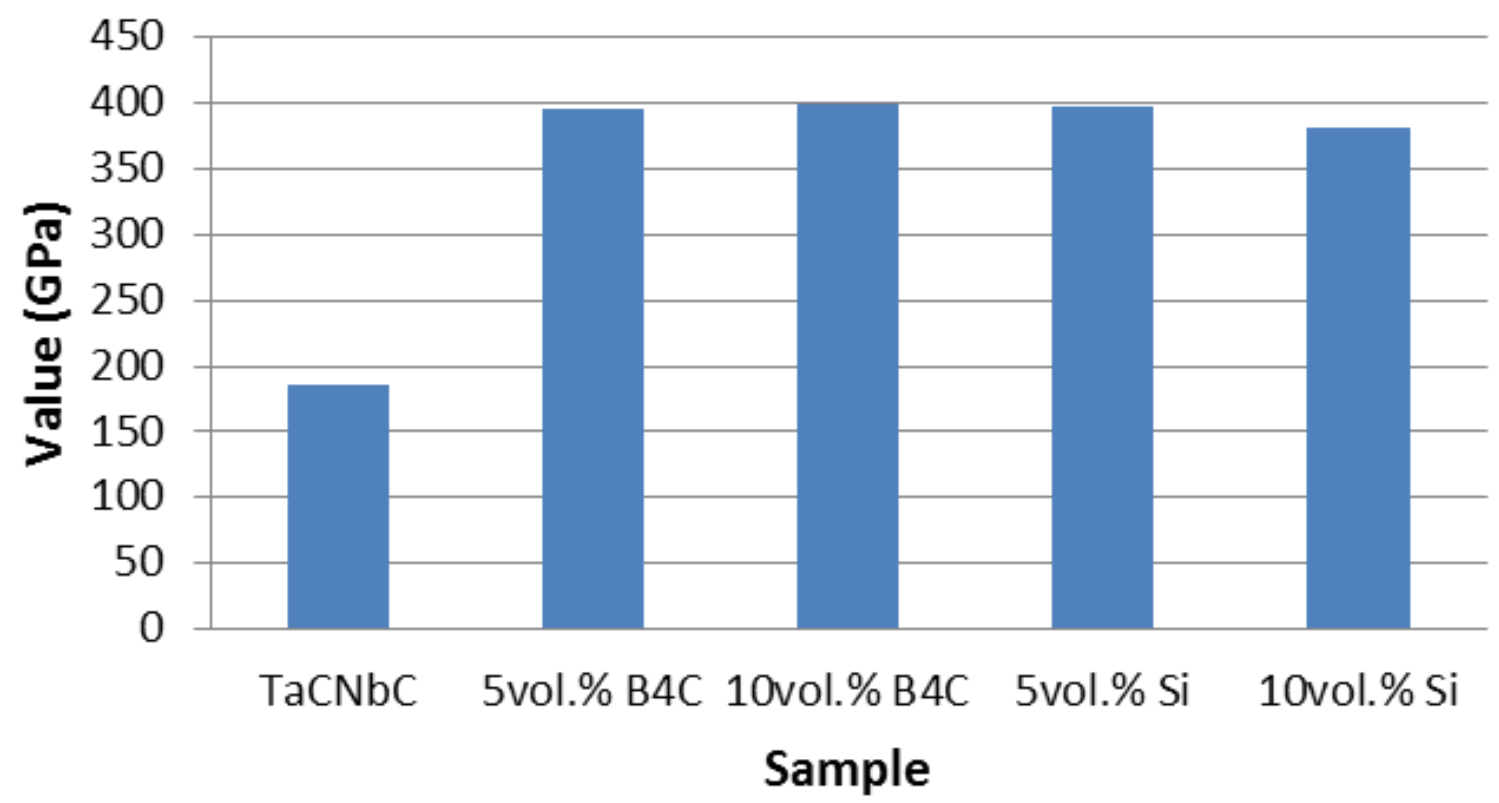

Figure 45: Elastic modulus measurements using 5 and 10 vol.\% sintering aid addition. 


\section{Hardness}

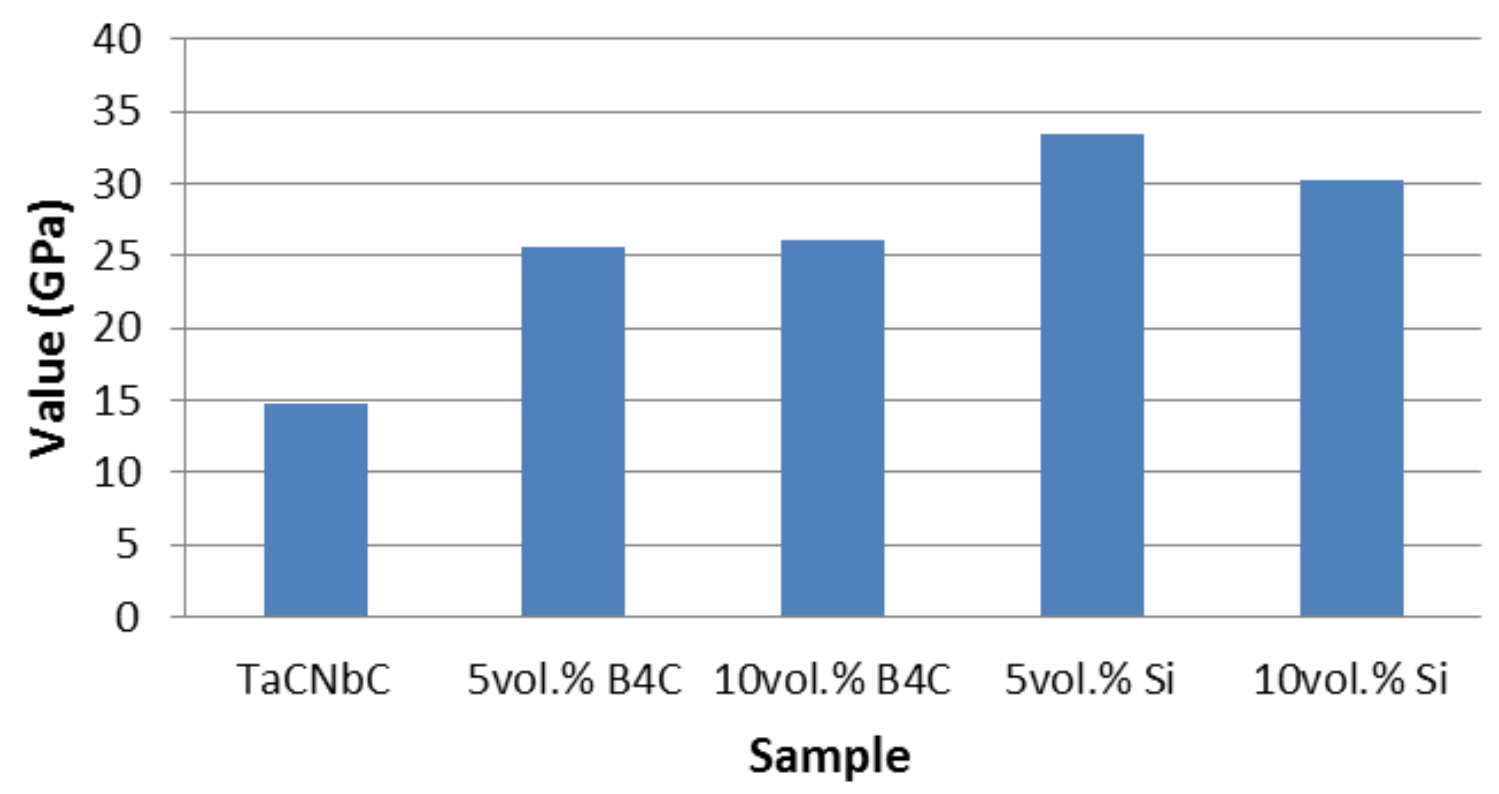

Figure 46: Hardness measurements using 5 and 10 vol.\% sintering aid addition.

\subsubsection{SPS Processing Parameters}

Initially, samples were made using parameters previously used by our group to consolidate $\mathrm{TaC}$ powder by SPS. These included a temperature of $1850^{\circ} \mathrm{C}, 60 \mathrm{MPa}$ pressure, a $100^{\circ} \mathrm{C} / \mathrm{min}$. heating rate, and a dwell time of 10 minutes. Table 9 shows the densification and grain size results of varying different processing parameters when using pure $\mathrm{TaC}-\mathrm{NbC}$ powder. When increasing the temperature $50^{\circ} \mathrm{C}$ from $1850^{\circ} \mathrm{C}$ to $1900^{\circ} \mathrm{C}$ (samples 1 and 2, respectively), a decrease in relative density and a slight increase in average grain size was observed. It was determined that increasing the maximum temperature would only lead to increased grain growth and the possibility of greater pore entrapment leading to a decrease in densification. When dwell time was increased from 10 to 15 minutes (samples 1 and 3, respectively), there was again a decrease in relative density. When the heating rate was increased from $100^{\circ} \mathrm{C} / \mathrm{min}$. to $200^{\circ} \mathrm{C} / \mathrm{min}$. (samples 1 
and 4 , respectively), a decrease in densification was noticed which was attributed to a shorter time for sintering to take place. Due to the maximum force that the graphite dies could handle at these high temperatures, the maximum pressure that could be achieved for our current die setup is $100 \mathrm{MPa}$. When the pressure was increased from 60 to 100 MPa (samples 1 and 5, respectively), an increase in densification was observed (from 86.9 to $88.6 \%$ ).

Table 9: Evaluation of SPS processing parameters.

\begin{tabular}{|c|c|c|c|c|c|c|}
\hline Sample & $\begin{array}{c}\text { Temperature } \\
\left({ }^{\circ} \mathrm{C}\right)\end{array}$ & $\begin{array}{c}\text { Pressure } \\
(\mathrm{MPa})\end{array}$ & $\begin{array}{c}\text { Heating } \\
\text { Rate } \\
\left({ }^{\circ} \mathrm{C} / \mathrm{min} .\right)\end{array}$ & $\begin{array}{c}\text { Dwell } \\
\text { Time } \\
(\mathrm{min} .)\end{array}$ & $\begin{array}{c}\text { Relative } \\
\text { Density } \\
(\%)\end{array}$ & $\begin{array}{c}\text { Avg. Grain } \\
\text { Size }(\mu \mathrm{m})\end{array}$ \\
\hline 1 & 1850 & 60 & 100 & 10 & 86.9 & $0.78 \pm 0.18$ \\
\hline 2 & 1900 & 60 & 100 & 10 & 85.0 & $0.79 \pm 0.20$ \\
\hline 3 & 1850 & 60 & 100 & 15 & 83.3 & $0.73 \pm 0.18$ \\
\hline 5 & 1850 & 60 & 200 & 10 & 84.4 & $0.78 \pm 0.16$ \\
\hline
\end{tabular}

It was decided that this increase in densification did not justify the use of the high pressures that would cause the dies to develop micro-cracks and fail. Therefore, in order to increase densification of the ceramic samples, sintering additives would be used at the maximum recommended pressure of the graphite dies $(60 \mathrm{MPa})$. Therefore the parameters that would be used for each of the $\mathrm{TaC}-\mathrm{NbC}$ ceramic composites would be a temperature of $1850^{\circ} \mathrm{C}$, pressure of $60 \mathrm{MPa}$, heating rate of $100^{\circ} \mathrm{C} / \mathrm{min}$., and a dwell time 
that would be dependent on the composition of the powder (which sintering additive was being used).

Samples of each composition were initially made with a dwell time of 10 minutes. The instantaneous relative densification curves were then analyzed and the dwell time was refined to achieve maximum relative density with a refined microstructure. Based on our evaluation of when densification was taking place, it was determined that for pure TaC-NbC samples as well as TaC-NbC samples with $\mathrm{B}_{4} \mathrm{C}$ as the sintering aid, a full dwell time of 10 minutes was required as densification continued throughout the entire dwell time. When Si was added as the sintering aid, it was noticed that no further densification occurred after about a minute and a half into the dwell time. Therefore it was decided that a dwell time of 3 minutes would be used to ensure maximum relative densification be achieved while limiting the time at high temperatures which promotes grain growth. This is further outlined in section 4.3.2.

\subsection{Use of Sintering Additives for Full Relative Densification}

As previously stated, a major challenge associated with UHTC materials is poor consolidation and grain growth. When a material is used in such extreme conditions, any defect areas (porosity, large grains, agglomerations, etc.) are likely to be the source of failure, significantly hindering the reliability of the composite. Either high mechanical pressures (>255 MPa) during sintering or the use of sintering additives can lead to full relative densification. The use of sintering additives can lead to the reduction in temperature when densification begins to take place, leading to increased final densification while also inhibiting grain growth. In this section, processing parameters 
and the use of sintering additives to achieve full relative densification (>99\%) will be discussed.

\subsubsection{Sample Synthesis}

Tantalum-Niobium carbide powder (TaC20NbC; 80 wt.\% TaC, 20 wt.\% NbC) $\left(\mathrm{Ta}_{0.67} \mathrm{Nb}_{0.33}\right) \mathrm{C}$ was consolidated by spark plasma sintering (SPS) with and without the addition of sintering additives. The sintering additives investigated $\left(\mathrm{B}_{4} \mathrm{C}\right.$ and $\left.\mathrm{Si}\right)$ were chosen based on their high temperature properties when considering the possible secondary phases formed during the sintering process. With the addition of $\mathrm{Si}$, the formation of $\mathrm{SiC}$ is expected which has a melting point $>2800^{\circ} \mathrm{C}$. With the addition of $\mathrm{B}_{4} \mathrm{C}, \mathrm{TaB}_{2}$ and $\mathrm{NbB}_{2}$ have the possibility to form which have melting points $>3030^{\circ} \mathrm{C}$.

The powder was prepared using tip sonication to mix the sintering additive with the TaC-NbC powder. The dried powder was loaded into graphite dies and sintered by SPS at $1850^{\circ} \mathrm{C}$ with a heating rate of $100^{\circ} \mathrm{C} /$ minute. The pure $\mathrm{TaC}-\mathrm{NbC}$ sample and the sample with $\mathrm{B}_{4} \mathrm{C}$ as sintering additive had a sintering dwell time of 10 minutes. The addition of Si led to faster densification and therefore only required a dwell time of 3 minutes to achieve a relative density $>99 \%$.

\subsubsection{Density, Microstructure, and Secondary Phase Formations}

Previous studies on the densification of UHTCs by SPS have reported that increasing the pressure during sintering will increase the densification. Bakshi et al. [37] were able to achieve full densification when spark plasma sintering $\mathrm{TaC}$ at $1850^{\circ} \mathrm{C}$ when using pressures of $255 \mathrm{MPa}$ and up. To study the effect of pressure during sintering, a TaC-NbC sample was sintered at $100 \mathrm{MPa}$, which is the maximum pressure attainable 
with our current SPS setup. While the increased pressure did result in an increase in relative density, it was only a slight rise to $88.6 \%$ compared to the $86.9 \%$ for the sample sintered at $60 \mathrm{MPa}$ as shown in Table 10. Since the increased pressure did not provide the desired densification, the emphasis of this study is on using sintering additives for improved densification.

Table 10: Properties of sintered compacts of $\mathrm{TaC}-\mathrm{NbC}$ with and without sintering additives prepared by SPS at $1850^{\circ} \mathrm{C}$ and a heating rate of $100^{\circ} \mathrm{C} / \mathrm{min}$.

\begin{tabular}{|c|c|c|c|c|c|}
\hline \multirow[b]{2}{*}{ Sample } & \multicolumn{2}{|c|}{ SPS Parameters } & \multicolumn{2}{|c|}{ Density $\left(\mathrm{g} / \mathrm{cm}^{3}\right)$} & \multirow{2}{*}{$\begin{array}{c}\text { Average } \\
\text { Grain Size } \\
(\mu \mathrm{m})\end{array}$} \\
\hline & $\begin{array}{l}\text { Pressure } \\
\text { (MPa) }\end{array}$ & $\begin{array}{l}\text { Hold } \\
\text { Time } \\
\text { (min.) }\end{array}$ & Actual & Relative & \\
\hline $\begin{array}{l}\text { TaC-NbC } \\
\text { Powder }\end{array}$ & N/A & N/A & 12.36 & $100.0 \%$ & $0.80 \pm 0.26$ \\
\hline $\mathrm{TaC}-\mathrm{NbC}$ & 60 & 10 & 10.75 & $86.9 \%$ & $0.78 \pm 0.18$ \\
\hline $\begin{array}{c}\text { TaC-NbC-100 } \\
\mathrm{MPa}\end{array}$ & 100 & 10 & 10.95 & $88.6 \%$ & $0.85 \pm 0.22$ \\
\hline $\begin{array}{l}\text { TaC-NbC-5 } \\
\text { vol. } \% \mathrm{~B}_{4} \mathrm{C}\end{array}$ & 60 & 10 & 11.71 & $99.1 \%$ & $0.83 \pm 0.15$ \\
\hline $\begin{array}{c}\text { TaC-NbC-5 } \\
\text { vol.\% Si }\end{array}$ & 60 & $3 *$ & 11.89 & $99.3 \%$ & $2.26 \pm 0.74$ \\
\hline
\end{tabular}

*Hold time was changed to 3 minutes as most of the densification occurred in that period. 
Based on the composition of the powder (which sintering additive was being used), the SPS processing parameters were refined.

In order to better understand when densification is taking place, the instantaneous relative density throughout the SPS process is calculated and shown in Figure 47. Instantaneous relative density is calculated based on the ram displacement data from the SPS process after subtracting out the displacement due to thermal expansion of the dies and the following equation.

$$
\rho_{\text {instantaneous }}=\left(\frac{L_{f}}{L}\right) \rho_{f}
$$

In order to calculate the thickness of the specimen (L) at time $t$ throughout the SPS process, the initial thickness of the powder is first found by adding the total distance the rams travel throughout the process to the final thickness $\left(\mathrm{L}_{\mathrm{f}}\right)$ of the SPS compact measured after sintering. From the initial thickness, the displacement of the ram is subtracted in order to get the instantaneous thickness at time t. The ratio of the final thickness to the instantaneous thickness is then multiplied by the final relative density $\left(\rho_{\mathrm{f}}\right)$ of the sample.

For the addition of 5 vol.\% $\mathrm{Si}$ as a sintering additive, a 10 minute hold achieved an almost full relative densification, however it was accompanied with large grain growth (average size of $3.1 \mu \mathrm{m}$ ). When grain coarsening occurs in UHTCs the mechanical properties are detrimentally effected compared with ceramics with refined microstructures [68]. By analyzing the instantaneous relative density during the SPS 
process it was observed that the sample reached its greatest density after about a minute and a half into hold time at $1850^{\circ} \mathrm{C}$ as seen in Figure 47.

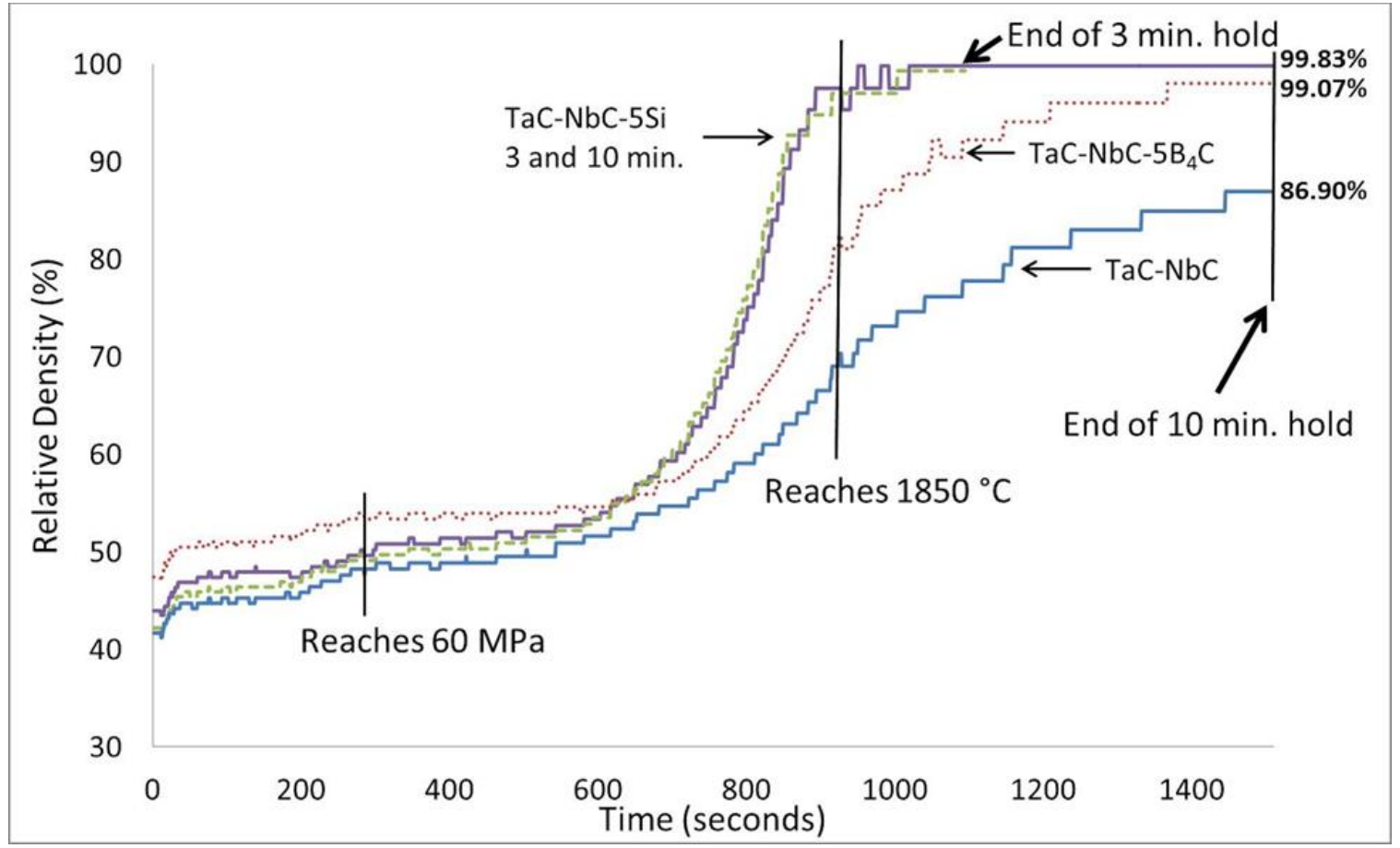

Figure 47: Instantaneous relative densities during the spark plasma sintering process.

Based on this information, a new sample was sintered with a 3 minute hold at $1850^{\circ} \mathrm{C}$ which was chosen in order to ensure the desired densification be reached while limiting the undesired grain growth occurrence that is attributed to the additional holding time. By reducing the hold time from 10 minutes to 3 minutes, grain growth occurrence was reduced from an average $3.1 \mu \mathrm{m}$ to $2.2 \mu \mathrm{m}$ while maintaining $99 \%$ or greater relative density (Figure 48). 

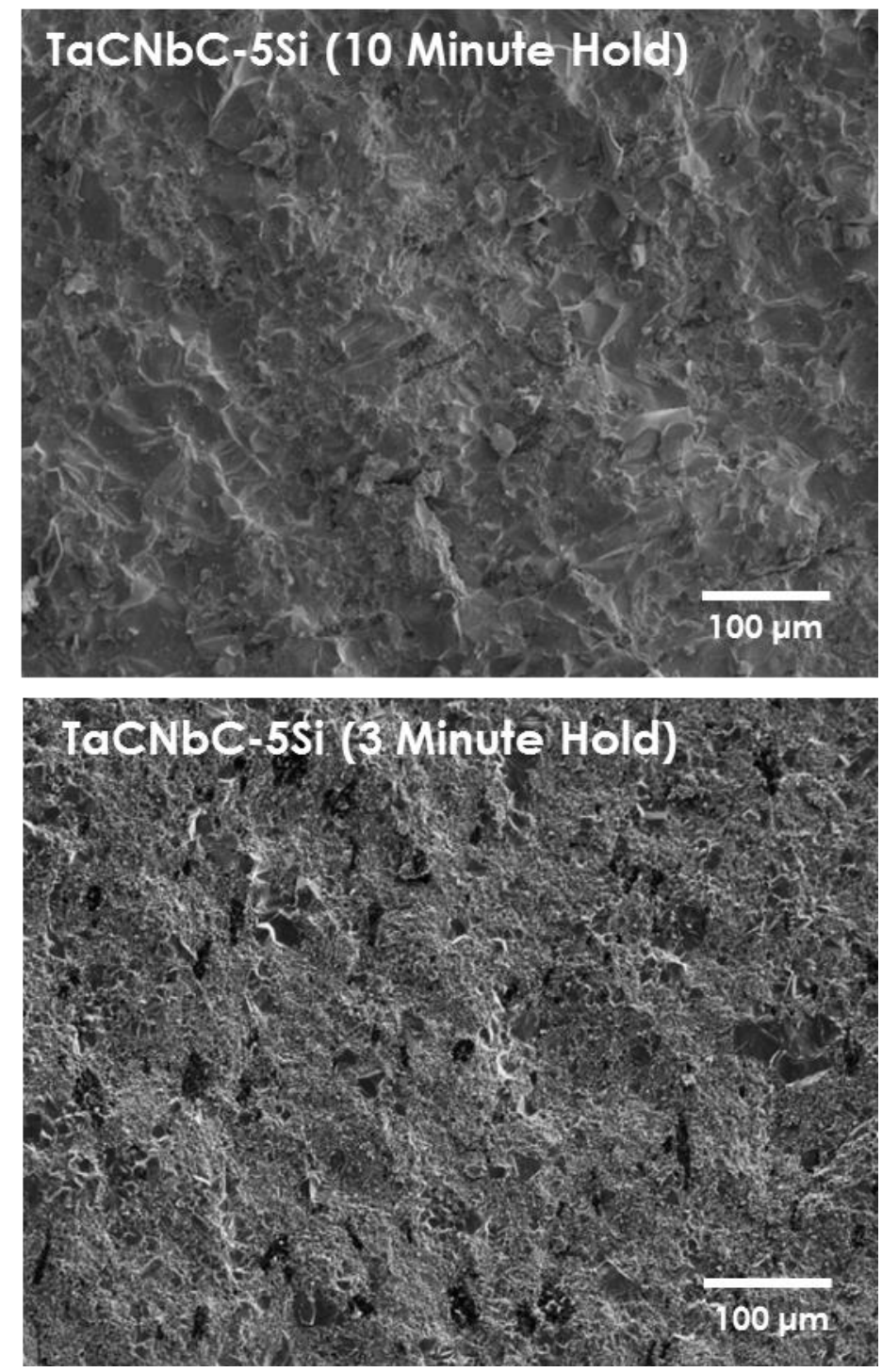

Figure 48: SEM images of the fracture surfaces of TaC-NbC-5 vol.\% Si addition sintered with a 10 minute and 3 minute dwell time.

The results presented throughout the rest of this dissertation are for samples sintered with a 3 minute hold when silicon is used as the sintering additive. For the addition of 5 vol.\% $\mathrm{B}_{4} \mathrm{C}$ as the sintering additive, a holding time of 10 minutes was deemed necessary since densification continued occurring throughout the majority of the 10 minute hold. 
Figure 49 shows SEM fractographs of the sintered samples. For pure TaC-NbC, a relative density of roughly $87 \%$ was achieved with no grain growth. The fracture that occurred was mostly inter-granular in nature.
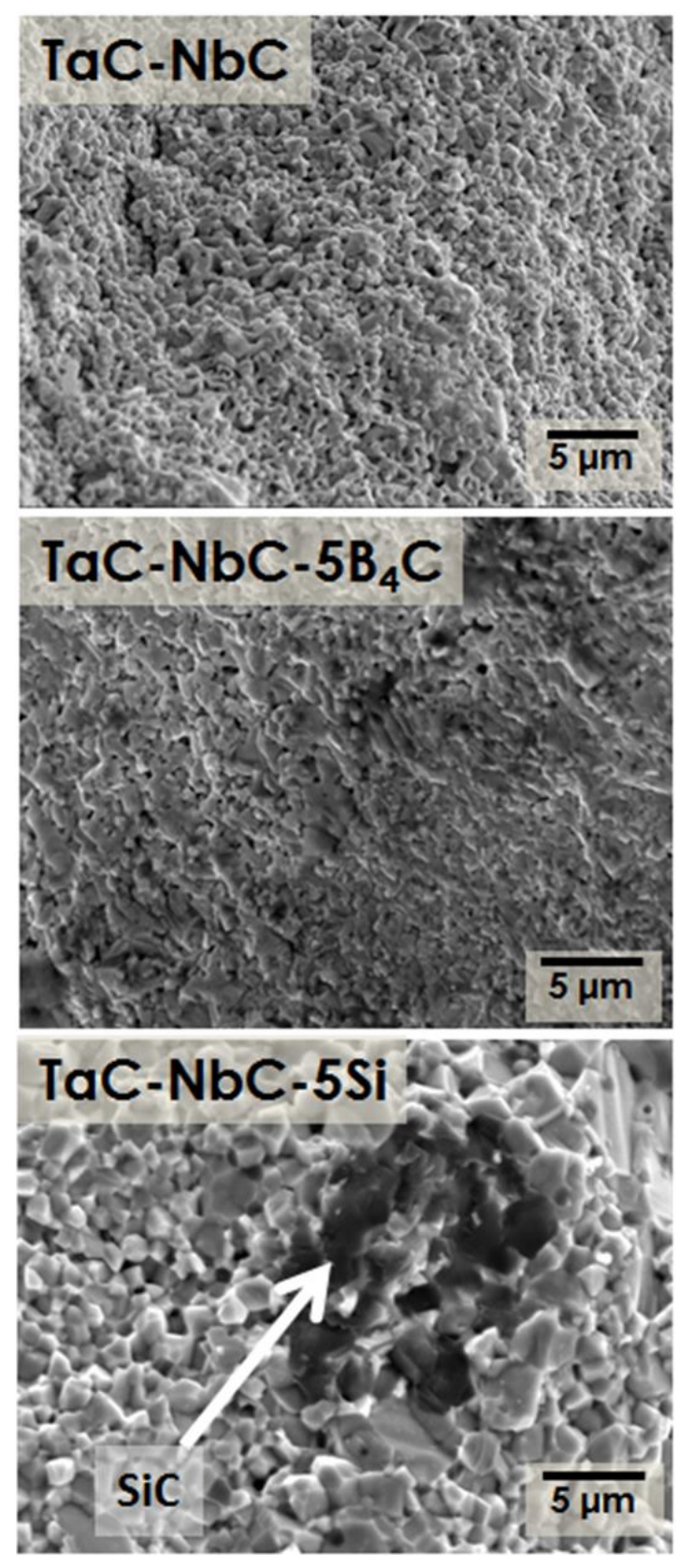

Figure 49: SEM micrographs of the fracture surfaces of $\mathrm{TaC}-\mathrm{NbC}$, $\mathrm{TaC}-\mathrm{NbC}-5 \mathrm{~B}_{4} \mathrm{C}$, and TaC-NbC-5Si prepared by SPS. 
For the samples with sintering additive, relative densities greater than $99 \%$ were achieved however some areas still showed localized porosity. These areas could be the areas with higher sintering additive concentration where faster consolidation occurred which resulted in the trapping of pores.

Figure 50 shows the cross-section of the sintered sample with 5 vol.\% Si addition achieved using a focused ion beam (FIB) to image the grains after rough milling followed by a precision milling cut. The sample sintered with 5 vol.\% Si addition had an average grain size of $2.2 \mu \mathrm{m}$ with a bi-modal size distribution where the majority of the grains had a size of about $1.5 \mu \mathrm{m}$ but also isolated areas where the grains had a size of about $3.5 \mu \mathrm{m}$. This could be due to an inhomogeneous distribution of the Si in the ceramic matrix. 


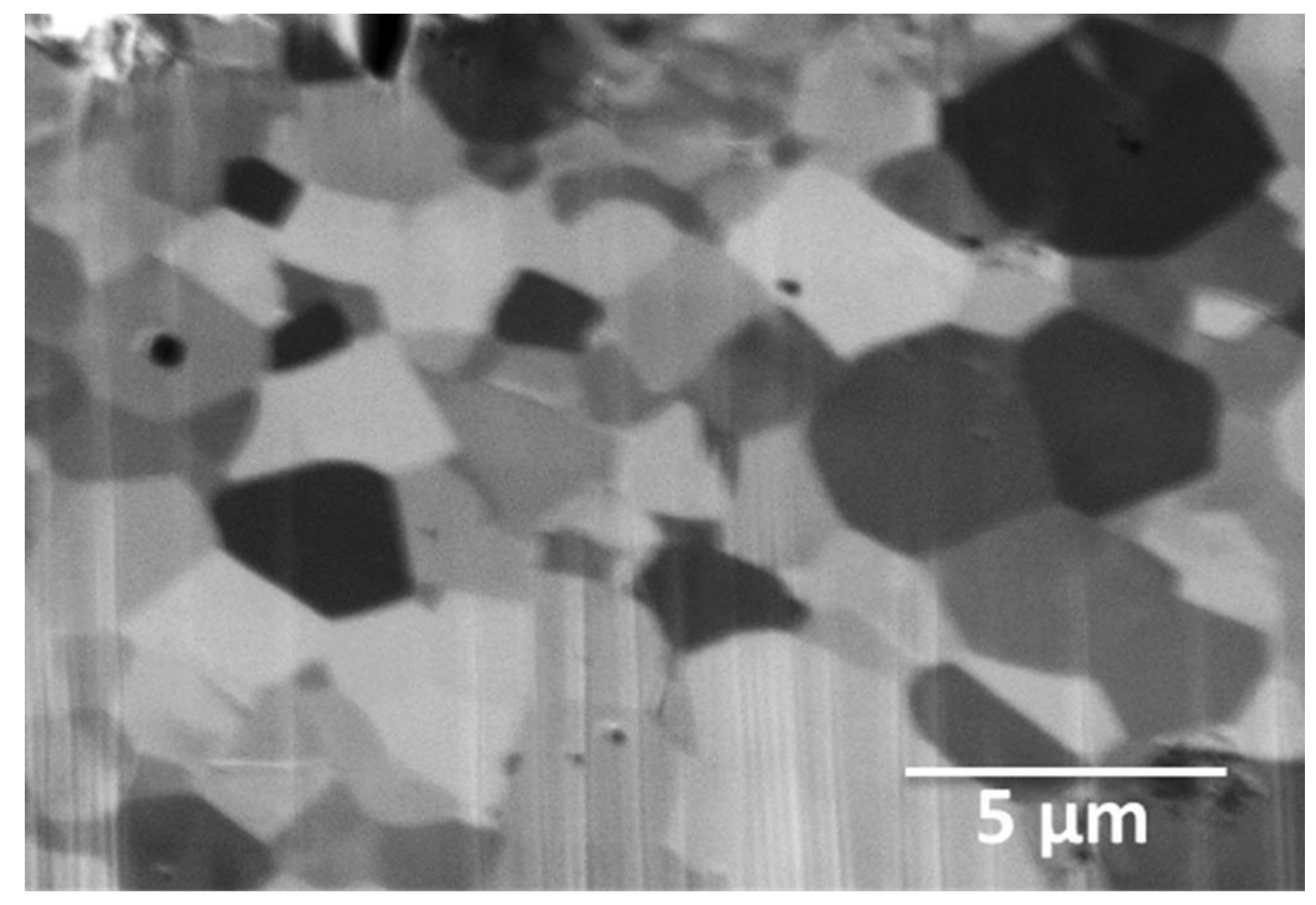

Figure 50: Representative cross-sectional image of sintered TaC-NbC-5 vol.\% Si showing dense microstructure and grains using focused ion beam after milling.

Figure 51 shows the XRD plots for the starting powders as well as the sintered samples. The starting powder of $\mathrm{TaC}-\mathrm{NbC}$ is not shown since the peak positions of the pre-sintered powder and sintered compact are the same. When 5 vol. $\% \mathrm{~B}_{4} \mathrm{C}$ is added to $\mathrm{TaC}-\mathrm{NbC}$, the new peaks belong to $\mathrm{TaB}_{2}$ and/or $\mathrm{NbB}_{2}$ which would be formed by the following reactions:

$$
\begin{aligned}
& 2 \mathrm{TaC}+\mathrm{B}_{4} \mathrm{C}=2 \mathrm{TaB}_{2}+3 \mathrm{C} \\
& 2 \mathrm{NbC}+\mathrm{B}_{4} \mathrm{C}=2 \mathrm{NbB}_{2}+3 \mathrm{C}
\end{aligned}
$$

It is not possible to distinguish between $\mathrm{TaB}_{2}$ and $\mathrm{NbB}_{2}$ using $\mathrm{XRD}$ since the peak positions are so similar (JCPDS: $\mathrm{TaB}_{2} 38-1462, \mathrm{NbB}_{2} 35-0742$ ). 


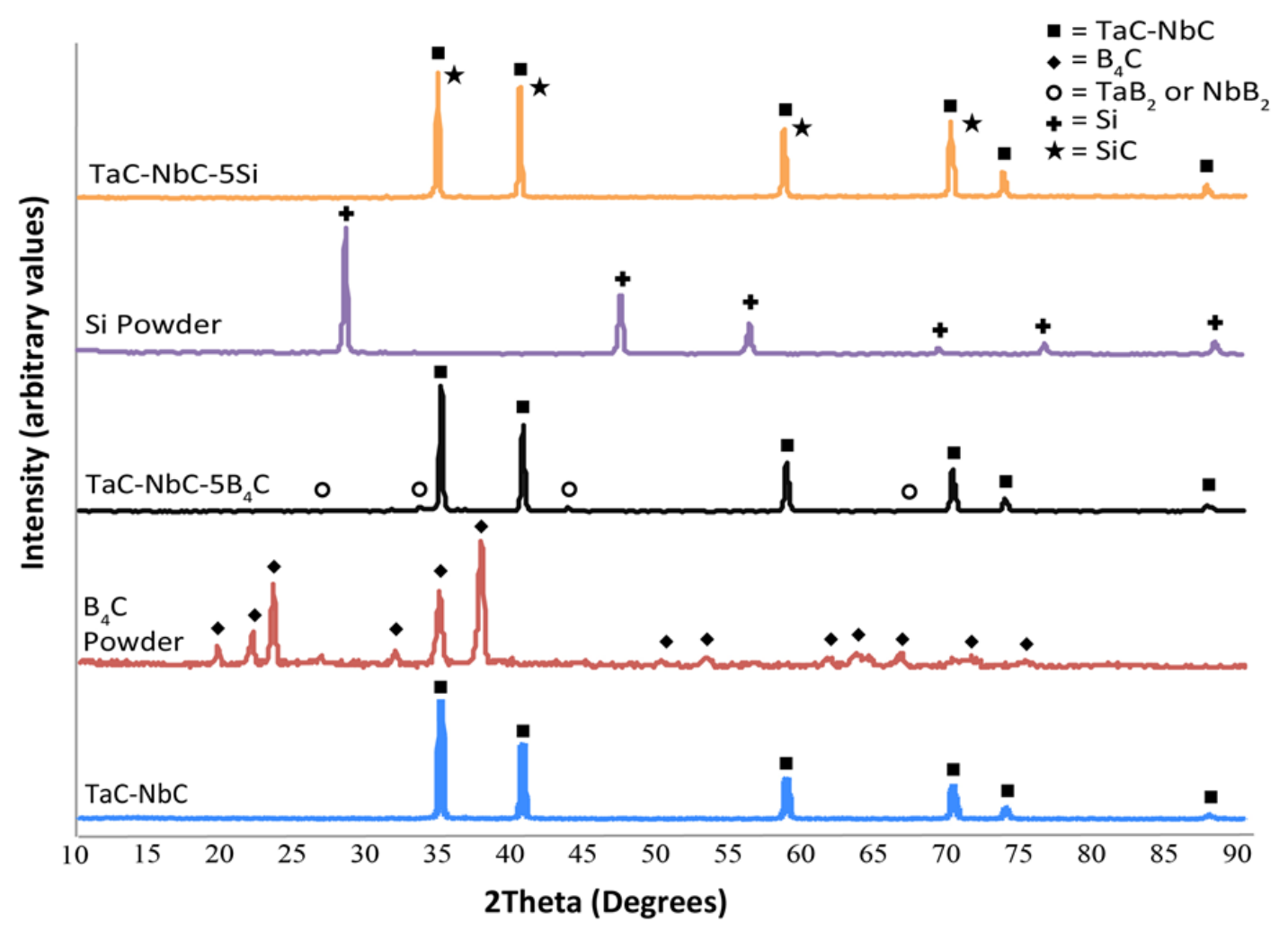

Figure 51: X-ray diffraction patterns for TaC-NbC composites with and without sintering additives after spark plasma sintering.

Since there are no $\mathrm{Si}$ peaks in the sintered sample, a secondary phase must be formed as a result of sintering TaC-NbC with 5 vol.\% Si addition. It is believed that $\beta$ $\mathrm{SiC}$ (shown in Figure 49) is formed which overlaps with the main peaks of the $\mathrm{TaC}-\mathrm{NbC}$ and is formed by the following reaction:

$$
\begin{aligned}
& \mathrm{TaC}+3 \mathrm{Si}=\mathrm{TaSi}_{2}+\mathrm{SiC} \\
& \mathrm{NbC}+3 \mathrm{Si}=\mathrm{NbSi}_{2}+\mathrm{SiC}
\end{aligned}
$$

There is a slight shift (about 0.08 degrees) to increasing $2 \theta$ values of the four main peaks of $\mathrm{TaC}-\mathrm{NbC}$ that provides evidence to this occurrence. Shifts in the other overlapping 
peaks were not noticeable which is to be expected due to the small amount of Si added to the system. While other researchers have seen the formation of $\mathrm{TaSi}_{2}$ as a result of the addition of $\mathrm{Si}$ in the $\mathrm{TaC}$ system, this was only seen when adding concentrations greater than 37 vol.\% $[39,69]$.

\subsubsection{Densification Process and Mechanisms of Sintering}

Figure 52 shows the SPS process outputs of temperature, chamber pressure, and ram pressure as well as the calculated relative instantaneous density of the $\mathrm{TaC}-\mathrm{NbC}$ sample with the addition of 5 volume $\% \mathrm{~B}_{4} \mathrm{C}$ as the sintering additive. The densification process can be split up into 6 stages (marked on figure) based on the slope of the densification curve with the most significant densification occurring in stages III-V.

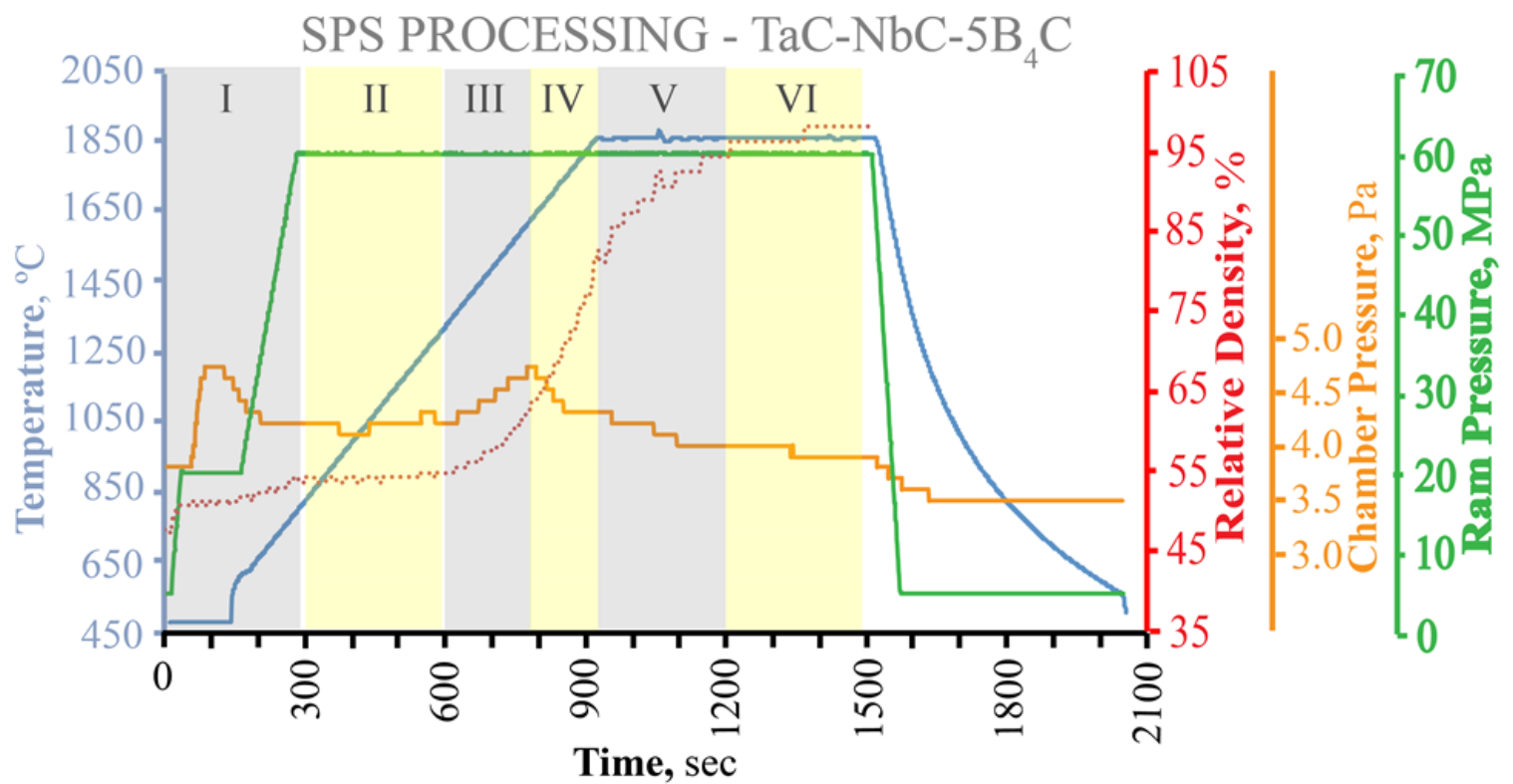

Figure 52: SPS processing and relative densification of $\mathrm{TaC}-\mathrm{NbC}$ with 5 vol. $\% \mathrm{~B}_{4} \mathrm{C}$ addition as a function of the sintering process. Stages of sintering (marked on figure as IVI) are the following: (I) Initial increase in SPS ram pressure, (II) continued ramping of temperature, (III) rapid densification begins accompanied with an increase in chamber pressure, (IV) rapid densification, (V and VI) final densification. 
In stage I, an increase in densification is observed from about $47 \%$ to $54 \%$ which is attributed to the increase in mechanical ram pressure from $5 \mathrm{MPa}$ to $60 \mathrm{MPa}$. The sharp increase in chamber pressure is a likely result of the release of trapped air around the powder which is forced out due to the increase in SPS ram pressure. Stage II, which is up to about $1330^{\circ} \mathrm{C}$, does not have any significant densification. Significant densification begins to occur at the beginning of stage III, encompassing a temperature range of $1330^{\circ} \mathrm{C}$ to $1630^{\circ} \mathrm{C}$. The increase in chamber pressure that is seen leads to the assumption that a reaction is occurring that releases a volatile substance. When hot pressing $\mathrm{TaC}$ with $\mathrm{B}_{4} \mathrm{C}$, Zhang et al. [30] saw the reduction of surface oxides which led to gas generation in the form of $\mathrm{B}_{2} \mathrm{O}_{3}$ and/or CO. However, the densification in stage III as well as the densification that takes place in stage IV could also be attributed to particle rearrangement and mass diffusion as well as a possible evaporation-condensation process [40]. In stage $\mathrm{V}$, starting from the hold temperature of $1850^{\circ} \mathrm{C}$ up until 5 minutes into the holding time, densification increased from $80 \%$ to $96 \%$. This can be attributed to the closing of pores. Stage VI only saw a small increase in densification which can be attributed to continued elimination of porosity.

Figure 53 shows the SPS process outputs of temperature, chamber pressure, and ram pressure as well as the calculated relative instantaneous density of the TaC-NbC sample with the addition of 5 volume $\% \mathrm{Si}$ as the sintering additive. This densification process can be split up into 6 stages (marked on figure) with the most significant densification occurring in stages III and IV. 


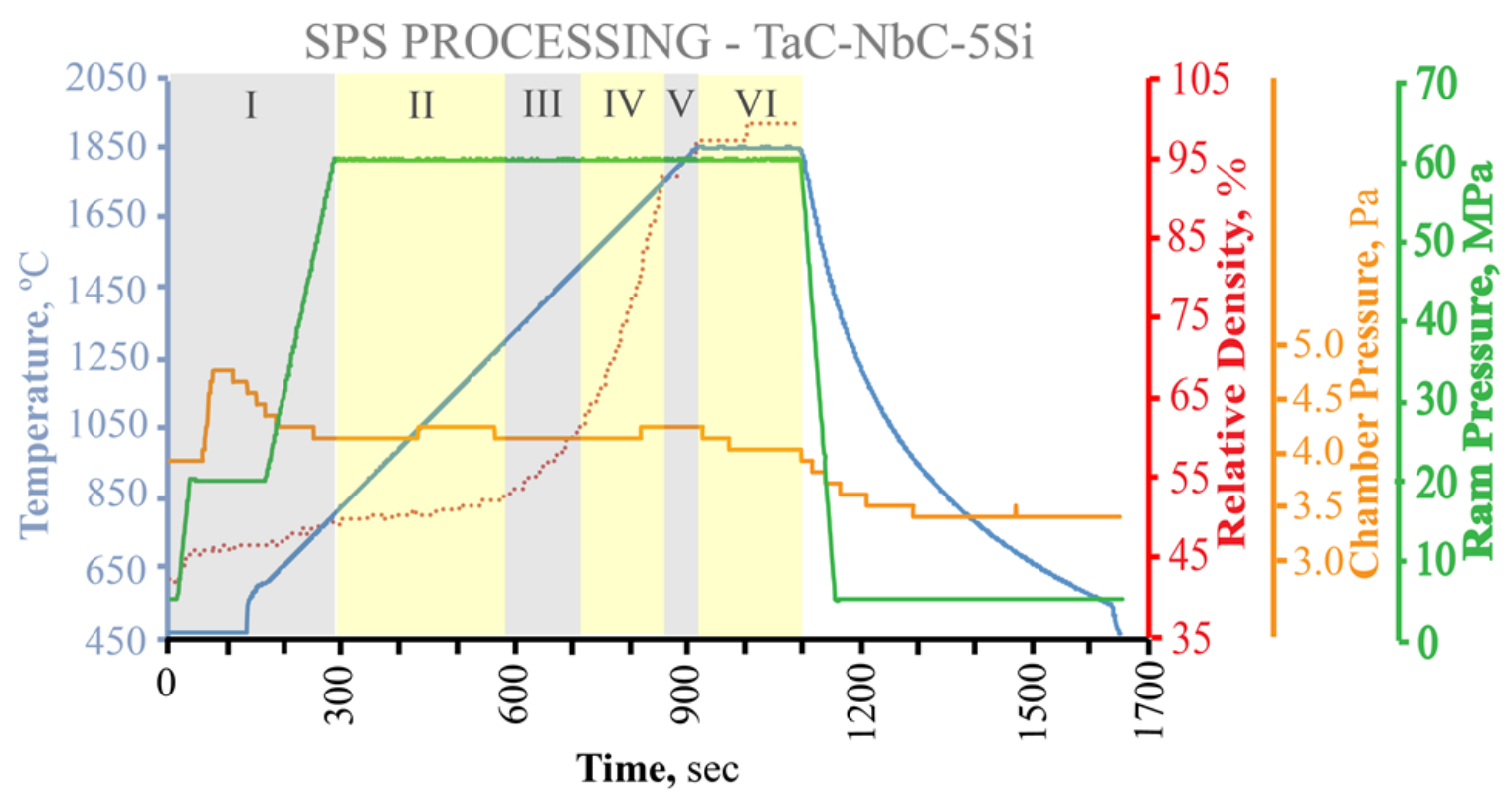

Figure 53: SPS processing and relative densification of $\mathrm{TaC}-\mathrm{NbC}$ with 5 vol.\% $\mathrm{Si}$ addition as a function of the sintering process. Stages of sintering (marked on figure as IVI) are the following: (I) Initial increase in SPS ram pressure, (II) continued ramping of temperature, (III) rapid densification begins, (IV) rapid densification, (V and VI) final densification.

Again, stage I densification is a result of the increase of mechanical ram pressure which results in greater powder packing and the release of trapped air. Stage II did not contain significant densification; however the increase in the chamber pressure could be an indication of a reaction between surface oxides and the silicon which would result in the release of $\mathrm{SiO}$ or $\mathrm{CO}$ gas [69]. Stages III and IV, taking place between $1287^{\circ} \mathrm{C}$ and $1725^{\circ} \mathrm{C}$, brings the beginning of significant densification. The densification mechanism attributed to these stages is particle rearrangement by creep flow up to $1413^{\circ} \mathrm{C}$ (melting point of $\mathrm{Si}$ ) and then wetting of the $\mathrm{TaC}-\mathrm{NbC}$ particles and the formation of $\mathrm{TaSi}_{2}$ and SiC. From the beginning of stage III to the end of stage IV, relative densification increased from about $53 \%$ to $92 \%$. It is assumed that the final densification, occurring in 
stages $\mathrm{V}$ and VI, is a result of the closing of pores as well as a possible evaporationcondensation mechanism that resulted in increased mass transportation and grain growth.

\subsubsection{High Load Instrumented Indentation}

Vickers hardness was measured by indenting on the polished cross-sections of the sintered samples. An increase in Vickers hardness is observed with the addition of sintering additives as shown in Table 11. Compared with the Vickers hardness of TaC$\mathrm{NbC}$ without any sintering additive, an increase in hardness of $188 \%$ and $194 \%$ was seen with the addition of $\mathrm{B}_{4} \mathrm{C}$ and $\mathrm{Si}$ as sintering additives, respectively. This is mainly contributed to the increase in densification of the sintered samples. Compared with results from other studies using $\mathrm{TaC}$ with sintering additives, the achieved hardness values were very similar. Zhang et al. [29] achieved a hardness value of $15.1 \mathrm{GPa}$ when adding a similar amount $(1 \mathrm{wt} . \%)$ of $\mathrm{B}_{4} \mathrm{C}$ to $\mathrm{TaC}$ and hot pressing at $2100^{\circ} \mathrm{C}$. Zhong et al. [69] showed a hardness of $13.2 \mathrm{GPa}$ when adding $0.78 \mathrm{wt} \% \mathrm{Si}$ to $\mathrm{TaC}$ and sintering by SPS at $1700^{\circ} \mathrm{C}$.

Table 11: Indentation properties of spark plasma sintered TaC-NbC compacts with and without sintering additives.

\begin{tabular}{|l|c|c|c|}
\hline \multicolumn{1}{|c|}{ Sample } & Hardness (GPa) & $\begin{array}{c}\text { Projected Damaged } \\
\left.\text { Area } \mathbf{( m m}^{\mathbf{2}}\right)\end{array}$ & Relative Density \\
\hline $\mathrm{TaC}-\mathrm{NbC}$ & $7.63 \pm 0.47$ & 1.020 & $86.90 \%$ \\
\hline $\mathrm{TaC}-\mathrm{NbC}-5 \mathrm{~B}_{4} \mathrm{C}$ & $14.32 \pm 0.59$ & 1.025 & $99.07 \%$ \\
\hline $\mathrm{TaC}-\mathrm{NbC}-5 \mathrm{Si}$ & $14.83 \pm 0.95$ & 0.555 & $99.32 \%$ \\
\hline
\end{tabular}

High load instrumented indentation was performed with an approximately $1 \mu \mathrm{m}$, $120^{\circ}$ conospherical diamond tip loaded across from the prepared sample. The 
indentations were performed under load control with a loading function of $100 \mathrm{~N} / \mathrm{min}$. up to $500 \mathrm{~N}$ followed by a dwell time of 5 minutes at $500 \mathrm{~N}$ before being unloaded at 100 N/min. The resulting load-displacement curves from high load instrumented indentation are shown in Figure 54.

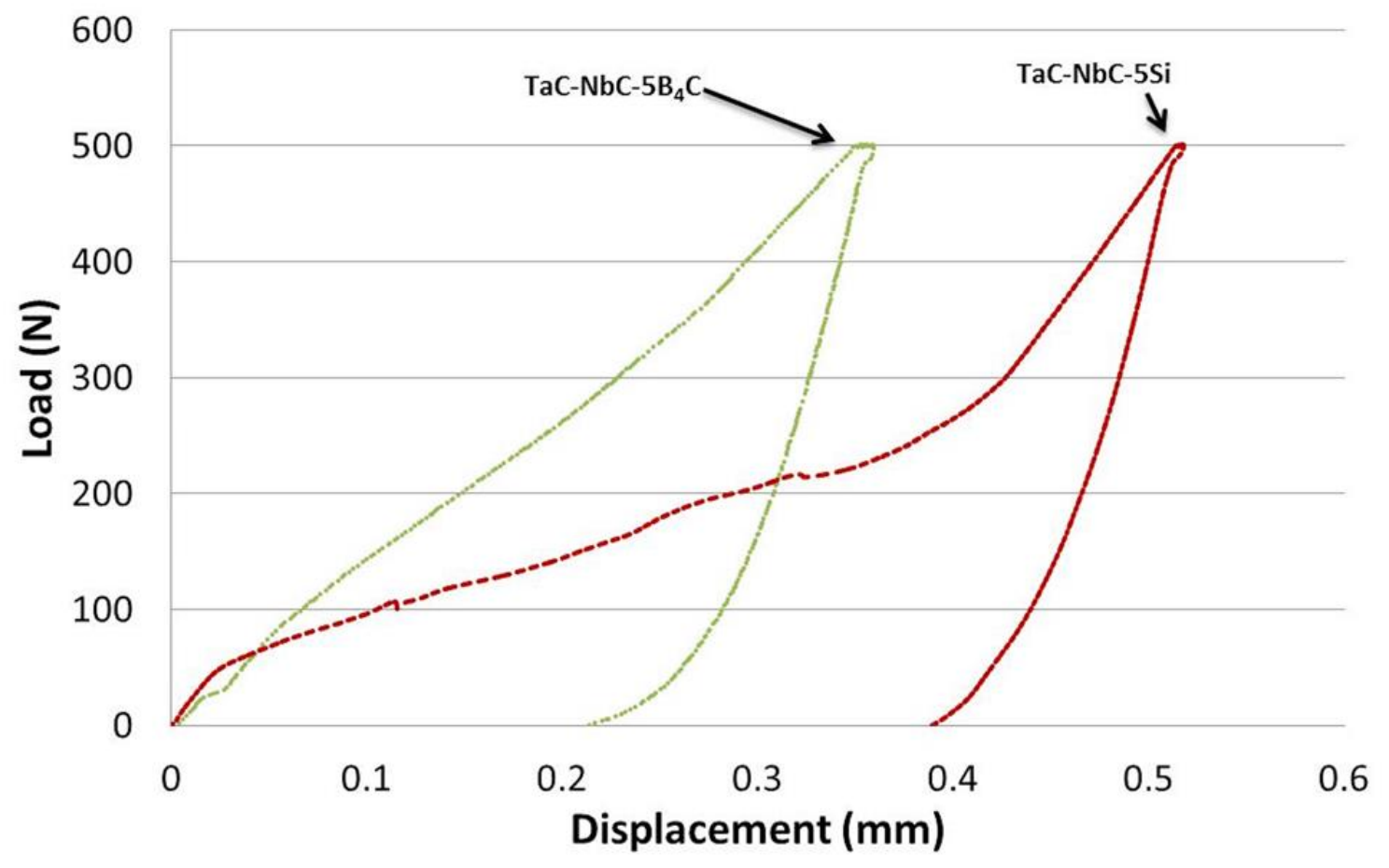

Figure 54: High load instrumented indentation load-displacement curves for $\mathrm{TaC}-\mathrm{NbC}$ with sintering additives.

Load-displacement curves are being shown only for the samples with sintering additives due to their similar density. In comparing the two curves with sintering aid addition, it is observed that a greater displacement is achieved to reach the same load when $\mathrm{Si}$ is the additive. When calculating the areas under the curves, the total work of indentation is $88.12 \mathrm{~mJ}$ for the sample with the addition of $5 \mathrm{vol} . \% \mathrm{~B}_{4} \mathrm{C}$ and $103.05 \mathrm{~mJ}$ for the sample with the addition of 5 vol.\% Si. Therefore the sample with the addition of 5 vol.\% Si addition dissipated $14.5 \%$ more energy during the indentation. 
Figure 55 shows the residual damage as a result of instrumented indentation at a high load of 500 N. In Figure 55a, the indenter tip impression can be clearly seen, however there are long cracks emanating out from the site of the indent that are not discernable at a low magnification. Therefore Figure 55b, which is a zoomed in image of the location marked in Figure 55a, is provided as a representative image showing the stemming cracks. Since the SEM images of the indentations cannot accurately capture the entire crack length of all cracks that have formed, a map of the residual damaged area (Figure 56) was made by tracing the crack lengths originating from point of indentation and connecting the ends of the cracks to calculate the projected area of residual damage as depicted in the schematic of Figure 55c. 

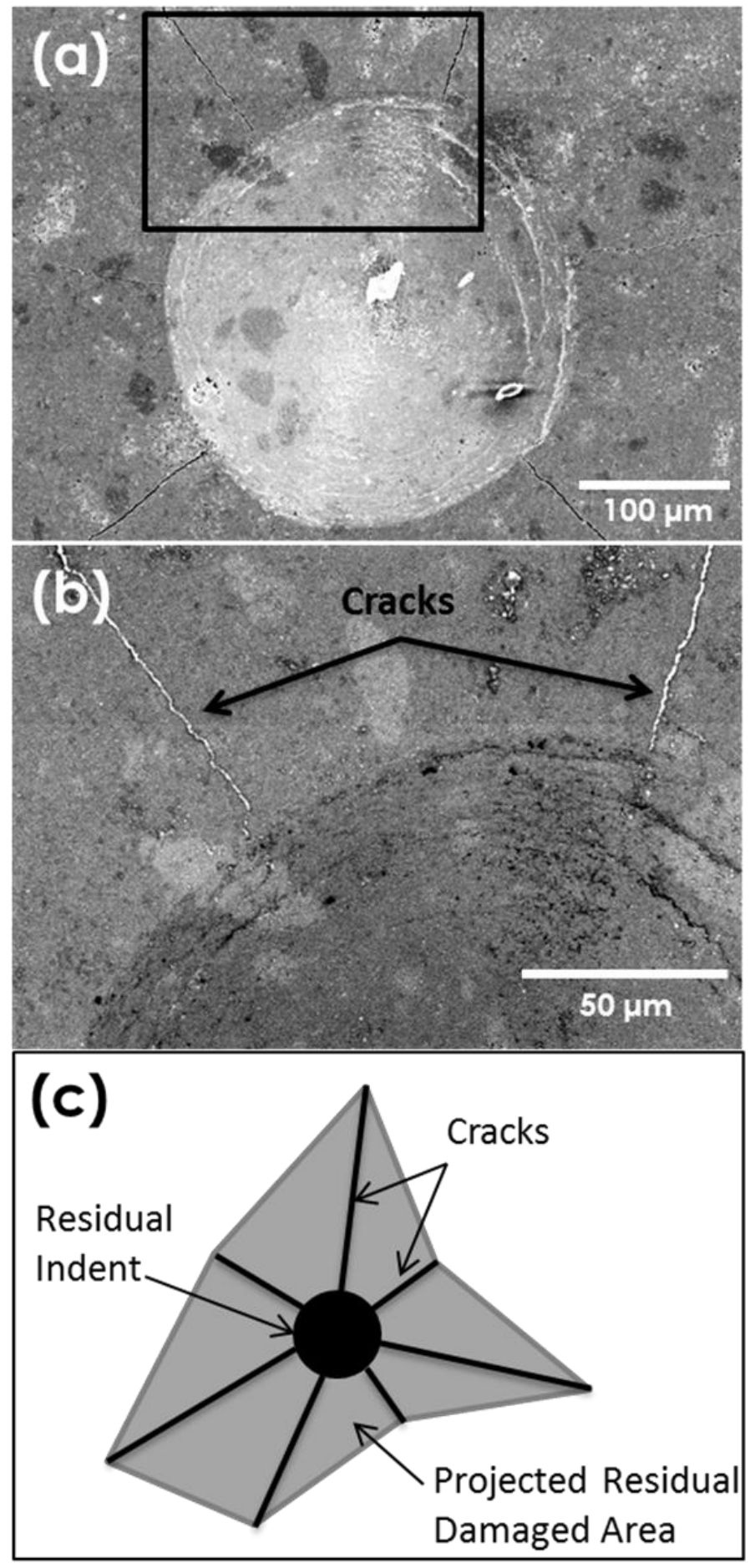

Figure 55: (a) SEM image of indentation impression on TaC-NbC, (b) zoomed in SEM image of area marked on (a) showing cracks protruding from indentation, and (c) a schematic of the cracks as a result of indentation with shaded projected residual damaged area. 


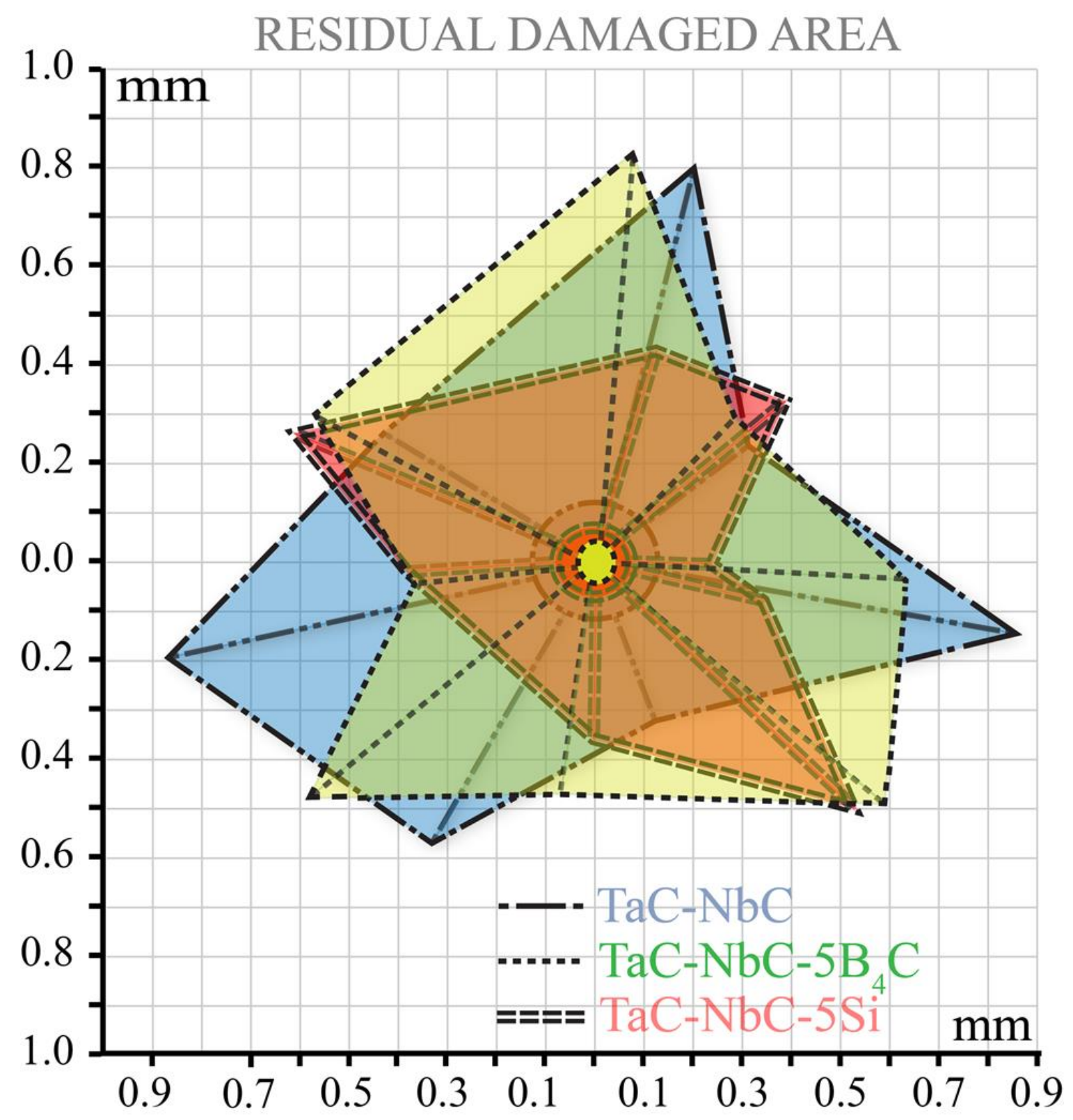

Figure 56: Map of projected residual damage resulting from high load instrumented indentation for spark plasma sintered $\mathrm{TaC}-\mathrm{NbC}$ with and without sintering additives.

The total projected area of residual damage (stated in Table 11) was very similar for the $\mathrm{TaC}-\mathrm{NbC}$ sample and the $\mathrm{TaC}-\mathrm{NbC}-5 \mathrm{~B}_{4} \mathrm{C}$ sample while the $\mathrm{TaC}-\mathrm{NbC}-5 \mathrm{Si}$ sample showed $\sim 46 \%$ less projected residual damaged area. This is in agreement with the finding from the load-displacement curves (Figure 54) that show the sample with 5 vol.\% Si 
dissipates a greater energy during indentation and therefore has a higher relative fracture toughness than the sample with the addition of 5 vol. $\% \mathrm{~B}_{4} \mathrm{C}$ addition. The increase in relative toughness with the addition of $\mathrm{Si}$ was expected since metals have a greater ability to plastically deform compared to ceramics and has been previously seen when $\mathrm{Si}$ or $\mathrm{TaSi}_{2}$ have been added to a $\mathrm{TaC}$ matrix. Zhong et al. [69] observed pullout of the $\mathrm{TaC}$ grains after fracturing indicating a weakened bonding between the $\mathrm{TaC}$ grains which instead favored toughening mechanisms such as interface debonding and crack deflection. Silvestroni et al. [70] also observed an increase in fracture toughness of $28 \%$ when 15 vol. $\% \mathrm{TaSi}_{2}$ was added to the $\mathrm{TaC}$ matrix, which was a result of the composite matrix being under a compressive stress. Hence, our finding are consistent with the same although with a novel high load $(500 \mathrm{~N})$ instrumented indentation technique on TaC-NbC based ceramics.

\subsubsection{Conclusions Based on the Use of Sintering Additives}

With the addition of just 5 vol. $\% \mathrm{~B}_{4} \mathrm{C}$ or 5 vol. $\% \mathrm{Si}$ as sintering additive, nearly full relative densification of the sintered $\mathrm{TaC}-\mathrm{NbC}$ is achieved with hold time as short as 3 minutes. The secondary phases formed by the addition of $\mathrm{B}_{4} \mathrm{C}$ as sintering aid are $\mathrm{TaB}_{2}$ and $\mathrm{NbB}_{2}$ which have melting points of $3040^{\circ} \mathrm{C}$ and $3050^{\circ} \mathrm{C}$, respectively. With the addition of $\mathrm{Si}$ as the sintering additive, the secondary phase formed is $\mathrm{SiC}$ with a melting point of $2830^{\circ} \mathrm{C}$. Each of the secondary phases formed have melting points that are high enough to allow the system to remain viable for use in high temperature applications. While the increase in hardness achieved using sintering additives is attributed to the increase in density, the use of $5 \mathrm{vol} . \%$ Si resulted in a $46 \%$ less projected residual damage 
area and $14.5 \%$ more energy dissipation during high load indentation than with the addition of 5 vol. $\% \mathrm{~B}_{4} \mathrm{C}$ and therefore has a higher overall relative toughness.

\subsection{Addition of GNP to Fully Dense TaC-NbC Composites}

Since near full relative densification $(>99 \%)$ has been achieved with the use of sintering additives and the intrinsic toughening mechanisms of bulk GNP have been studied with respect to orientation, what was learned is used to synthesize fully dense, GNP reinforced TaC-NbC composites with improved toughening. This section will look at the microstructure changes of the ceramic composites as a result of GNP addition. In addition, the toughening mechanisms will be examined in terms of all three hypothesized toughening regimes in the reinforced ceramic composites. Comparisons between the respective ceramic compositions with and without GNP addition will be assessed to realize the added benefits of GNP addition.

\subsubsection{Sample Synthesis}

Tantalum-Niobium carbide powder (TaC20NbC; 80 wt.\% TaC, 20 wt.\% NbC) $\left(\mathrm{Ta}_{0.67} \mathrm{Nb}_{0.33}\right) \mathrm{C}$ was consolidated with 3 vol.\% GNP addition with and without the addition of sintering additives $\left(5\right.$ vol. $\% \mathrm{~B}_{4} \mathrm{C}$ or $\left.\mathrm{Si}\right)$.

Each composite powder was prepared using tip sonication of the mixture in acetone for the sample powders prepared with GNP and $\mathrm{B}_{4} \mathrm{C}$ with GNP and in methanol for the powder prepared with Si and GNP to avoid any detrimental reactions. The dried powder was loaded into graphite dies and consolidated by spark plasma sintering under vacuum atmosphere at $1850^{\circ} \mathrm{C}$ and $60 \mathrm{MPa}$ pressure with a heating rate of $100^{\circ} \mathrm{C} /$ minute. 
A dwell time of 10 minutes was used for the TaC-NbC-3GNP and TaC-NbC-5B 4 C-3GNP samples and only 3 minutes for the TaC-NbC-5Si-3GNP sample to reduce grain growth.

\subsubsection{Density, Microstructure, and Secondary Phase Analysis}

The use of sintering additives improved the density from $87.3 \%$ to $98.7 \%$ when using 5 vol. $\% \mathrm{~B}_{4} \mathrm{C}$ and to $99.0 \%$ when using 5 vol. $\% \mathrm{Si}$ (Table 12).

Table 12: Properties of sintered compacts of TaC-NbC-3GNP with and without sintering additives prepared by SPS at $1850^{\circ} \mathrm{C}$ and $60 \mathrm{MPa}$ with a heating rate of $100^{\circ} \mathrm{C} / \mathrm{min}$.

\begin{tabular}{|c|c|c|c|c|}
\hline \multirow{2}{*}{ Sample } & \multirow{2}{*}{$\begin{array}{c}\text { SPS Hold } \\
\text { Time } \\
\text { (minutes) }\end{array}$} & Actual $\left(\mathrm{g} / \mathrm{cm}^{3}\right)$ & Relative & Average Grain \\
\cline { 3 - 4 } & 10 & 10.51 & $87.3 \%$ & $0.78 \pm 0.14$ \\
\hline TaC-NbC-3GNP & 10 & 11.44 & $98.7 \%$ & $1.53 \pm 0.29$ \\
\hline $\begin{array}{c}\text { TaC-NbC-5B } 4 \text { C- } \\
\text { 3GNP }\end{array}$ & 3 & 11.68 & $99.0 \%$ & $1.35 \pm 0.31$ \\
\hline $\begin{array}{c}\text { TaC-NbC-5Si- } \\
\text { 3GNP }\end{array}$ & & & & \\
\hline
\end{tabular}

Grain size measurements were calculated from focused ion beam (FIB) imaging after milling the sample with rough milling followed by precision milling in order to reveal the grain morphologies (Appendix 1). A total of 50 grain size measurements were gathered for each sample and the average grain sizes found are reported in Table 12. With the addition of GNP to $\mathrm{TaC}-\mathrm{NbC}$, an average grain size of $0.78 \mu \mathrm{m}$ was found which is slightly less than the calculated initial TaC-NbC powder size of $0.80 \mu \mathrm{m}$. This small decrease is within the error bounds of the grain size calculations. In both the samples with 
the addition of sintering additives, grain growth was observed. The largest grain growth was seen in the sample with $\mathrm{B}_{4} \mathrm{C}$ and GNP addition where the average grain size nearly doubled to $1.53 \mu \mathrm{m}$. It is possible this large grain growth can be attributed to the $\mathrm{B}_{4} \mathrm{C}$ powder, which had a bi-modal size distribution with grain sizes up to $5 \mu \mathrm{m}$ as well as the longer dwell time during sintering over the sample with Si addition. Figure 57, Figure 58, and Figure 59 show the fracture surfaces of TaC-NbC-3GNP, TaC-NbC-5B ${ }_{4} \mathrm{C}-3 \mathrm{GNP}$, and TaC-NbC-5Si-3GNP, respectively. In both the TaC-NbC-3GNP (Figure 57b) and TaC-NbC-5B ${ }_{4} \mathrm{C}-3 \mathrm{GNP}$ (Figure 58b) samples, the GNP is observed conforming to the TaC-NbC grains by bending, kinking, and wrapping around them. This results in greater interfacial contact between the GNP and the TaC-NbC matrix, which leads to an increased toughening potential due to the improved ability for stress to be transferred from the ceramic matrix to the GNP.

The sample with Si and GNP addition shown in Figure 59 achieved relative densification of $99 \%$ with a dwell time of just 3 minutes and had an increase in grain size of $173 \%$ over the sample without sintering additive. Compared to the TaC-NbC-5Si sample without GNP addition, which had an increase in grain size of $289 \%$, the addition of GNP led to a reduction in grain growth. Nieto et al. [21] previously documented a $60 \%$ reduction in grain growth of $\mathrm{TaC}$ grains with the addition of 3 and 5 vol.\% GNP. This reduction of grain growth was the result of the GNP wrapping around TaC grains to act as a diffusion barrier as well as the pinning of the $\mathrm{TaC}$ grains. 

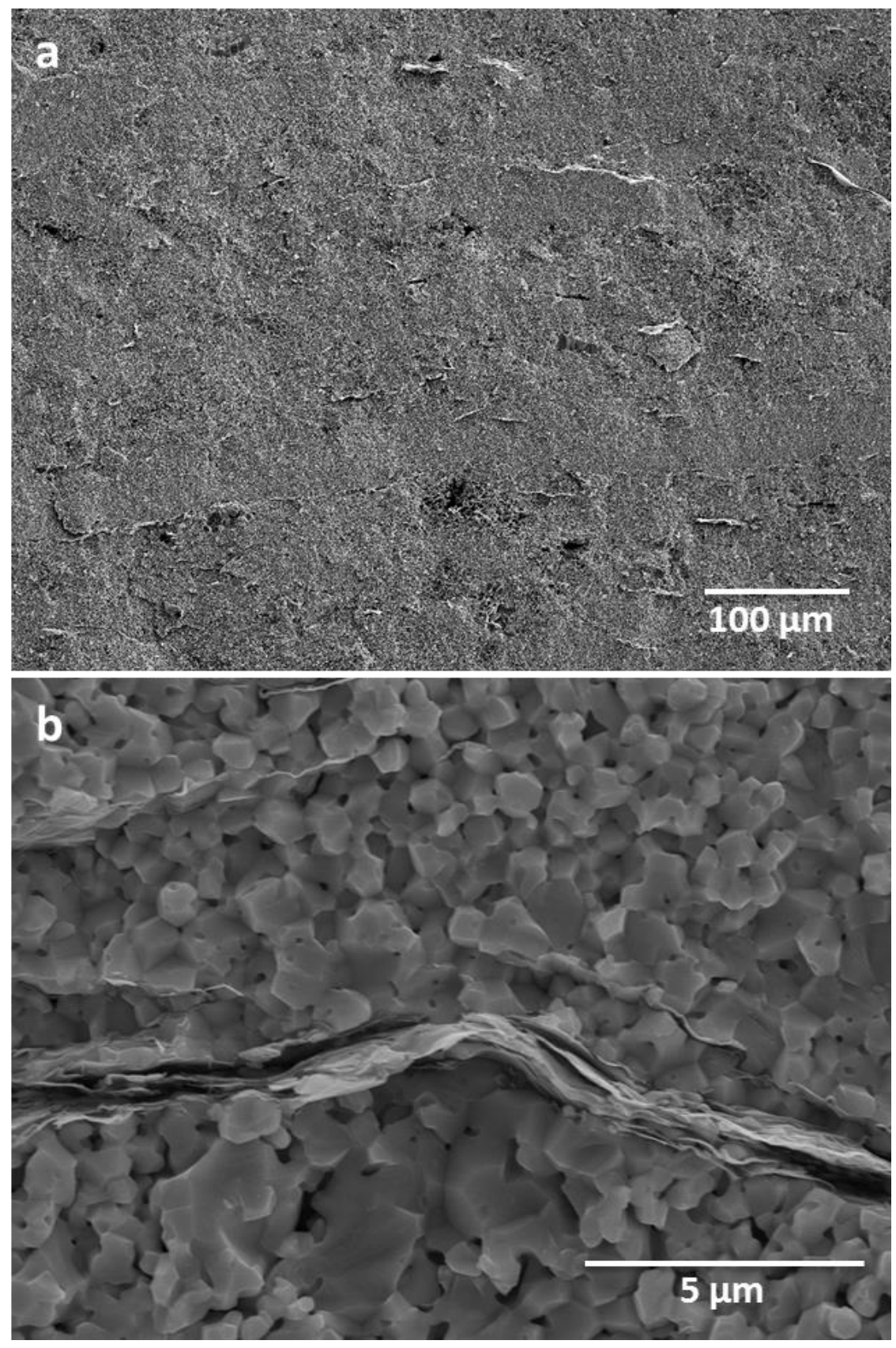

Figure 57: SEM micrographs of the fractured surfaces of TaC-NbC-3GNP: a) low magnification image showing alignment of the GNP, b) high magnification image showing interaction between GNP and matrix grains. 

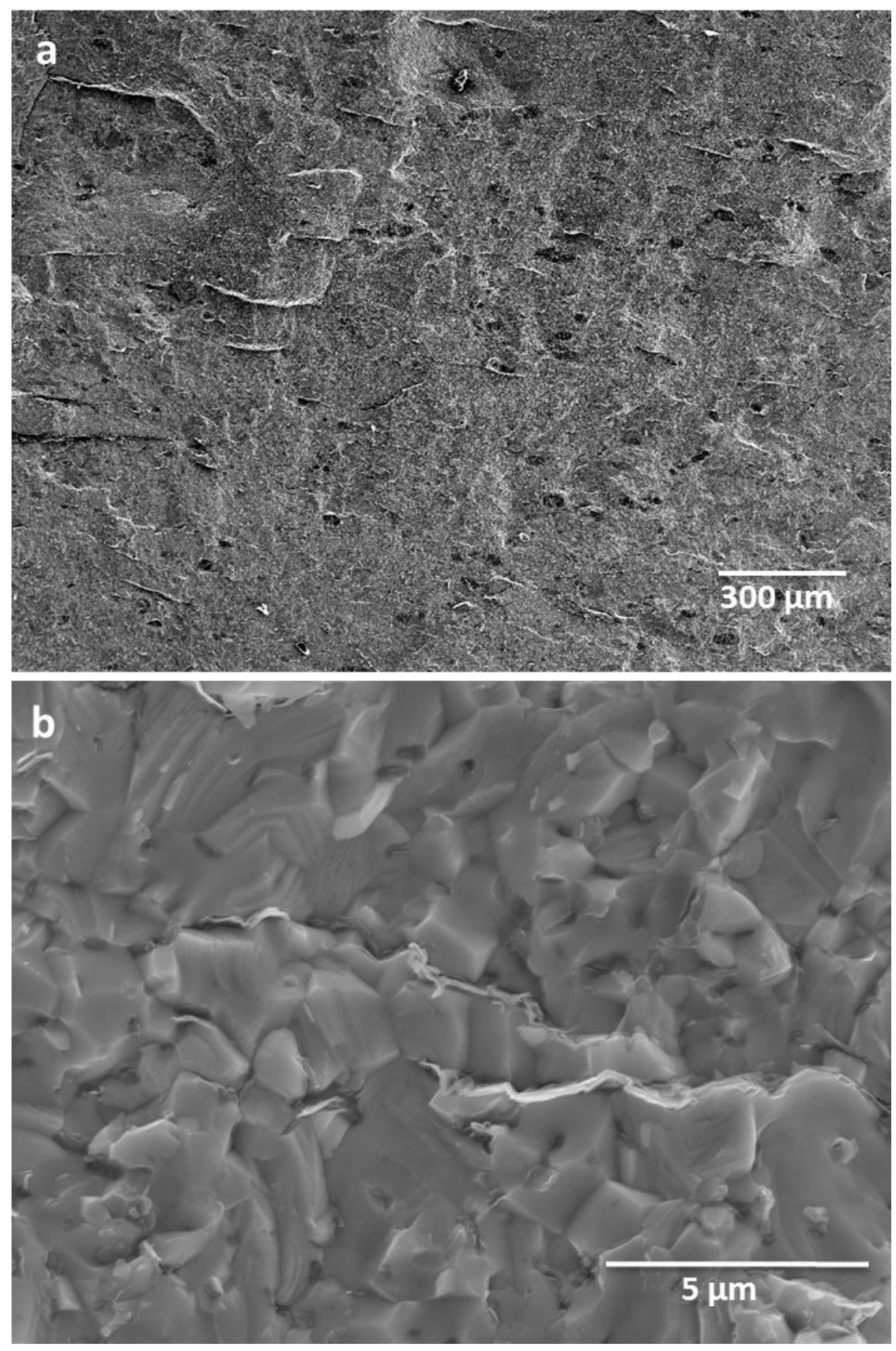

Figure 58: SEM micrographs of the fractured surfaces of TaC-NbC-5B ${ }_{4} \mathrm{C}-3 \mathrm{GNP}$ : a) low magnification image showing alignment of the GNP, b) high magnification image showing interaction between GNP and matrix grains.

Figure 57a and Figure 58a show low magnification images of the fracture surface of TaCNbC-3GNP and TaC-NbC-5B 4 C-3GNP, respectively. The GNP can be seen dispersed throughout the composite in an aligned orientation perpendicular to the pressing axis of 
the SPS machine. Previous publications from various researchers have also reported this alignment as a result of processing via SPS [21, 60, 63, 71].

In the sample with silicon as the sintering additive, the alignment of the GNP is not evident when compared with the other two samples. While the low magnification fractograph of the silicon addition sample, Figure 59a, does not display GNP alignment, areas of a secondary phase formation alignment are noticed. These areas of alignment, which are magnified in Figure 59b and Figure 59c, show a secondary phase of SiC which has been verified using Energy Dispersive Spectroscopy (EDS) (see appendix 2). The Si, which was added to aid in densification, is believed to have reacted with the already aligned GNP to form SiC which resulted in the visible alignment of the secondary phase. Gibbs free energy $(\Delta \mathrm{G})$ calculations indicate that the Si can react to form $\beta$-SiC, $\alpha-\mathrm{SiC}$, $\mathrm{TaSi}_{2}$, and $\mathrm{NbSi}_{2}$ as shown in the following reactions:

$$
\begin{aligned}
& \mathrm{Si}+\mathrm{C}=>\beta-\mathrm{SiC} ; \Delta \mathrm{G}=-274.7 \mathrm{~kJ} / \mathrm{mol} @ 1850^{\circ} \mathrm{C} \\
& \mathrm{Si}+\mathrm{C}=>\alpha-\mathrm{SiC} ; \Delta \mathrm{G}=-41.1 \mathrm{~kJ} / \mathrm{mol} @ 1850^{\circ} \mathrm{C} \\
& \mathrm{Ta}+2 \mathrm{Si}=>\mathrm{TaSi}_{2} ; \Delta \mathrm{G}=-82.1 \mathrm{~kJ} / \mathrm{mol} @ 1850^{\circ} \mathrm{C} \\
& \mathrm{Nb}+2 \mathrm{Si}=>\mathrm{NbSi}_{2} ; \Delta \mathrm{G}=-93.6 \mathrm{~kJ} / \mathrm{mol} @ 1750^{\circ} \mathrm{C}
\end{aligned}
$$

While the $\Delta \mathrm{G}$ calculations reveal that at the dwell temperature of $1850^{\circ} \mathrm{C}$ all of the reactions are possible, it is believed that the secondary phase formed is $\beta$-SiC which is the most favorable of the reactions. Although it is believed that $\beta$-SiC makes up most of the secondary SiC phase, the elongation of the $\mathrm{SiC}$ grains shown in Figure 59c point to a $\beta$ $\mathrm{SiC} \rightarrow \alpha-\mathrm{SiC}$ transformation. The transformation from the $\beta$-SiC to the hexagonal 
polytype has been extensively documented [72-74]. Sciti et al. [75] noted the development of elongated or platelet-shaped grains as a result of the $\beta \rightarrow \alpha$ phase transformation at temperatures of $1900-2000^{\circ} \mathrm{C}$. It is also possible that the elongated laminar structure of the GNP aids in the formation of elongated $\alpha$-SiC formation. Due to the shortened dwell time of just 3 minutes with this sample, it is expected that the elongated morphology will not be as prevalent as it would be with a longer reaction time. While the reaction between the GNP and the Si resulted in the deterioration of the GNP structure, areas of unreacted GNP aligned perpendicular to the SPS pressing axis were still noted. 

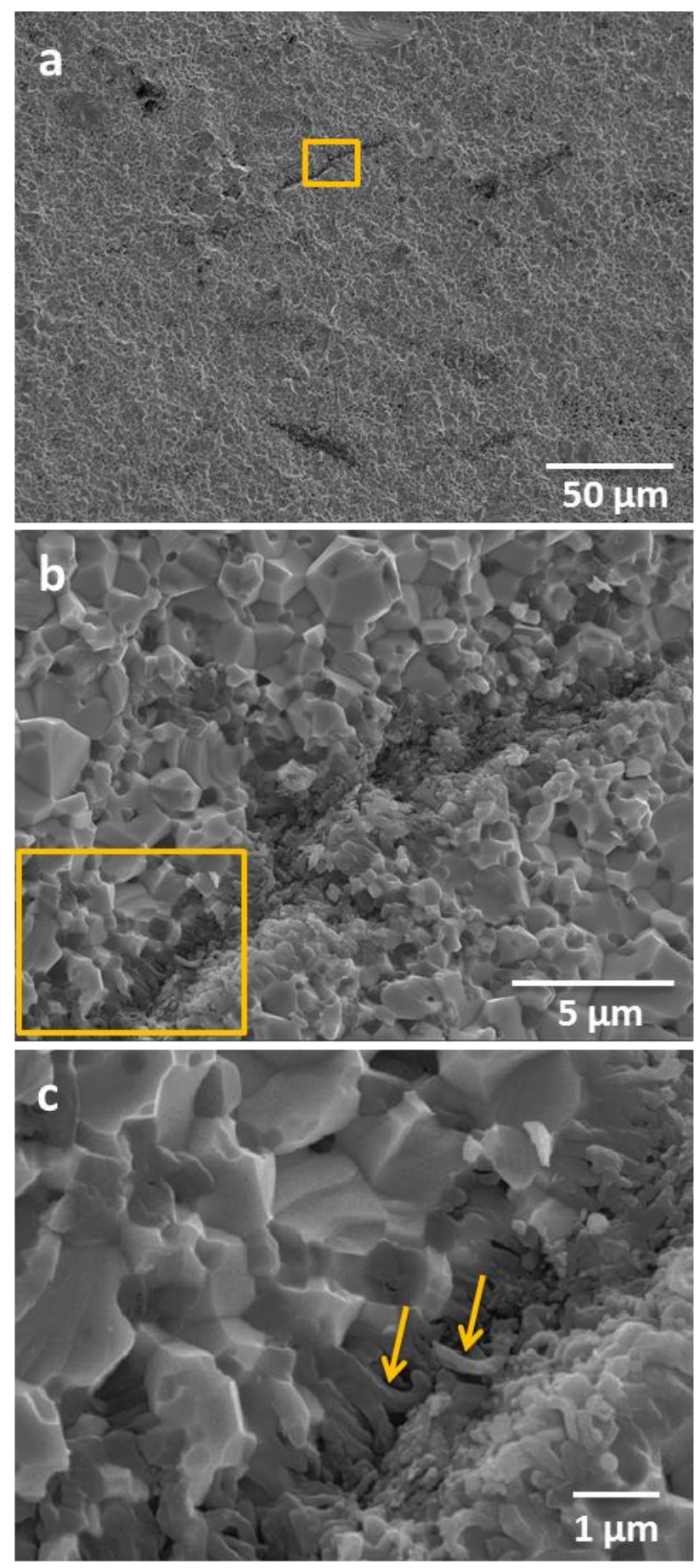

Figure 59: SEM migrographs of the fractured surfaces of TaC-NbC-5Si-3GNP sample showing a) low magnification view, b) magnified view of the area marked in (a), and c) magnified view of the area marked in (b) with arrows pointing to elongation of $\mathrm{SiC}$ grains. 
Figure 60 shows the XRD plots for the sintered compacts as well as for a sintered compact of pure $\mathrm{TaC}-\mathrm{NbC}$ for comparison. There are no new peaks formed in the TaCNbC-3GNP sample which was expected due to the low volume percentage of GNP added. When 5 vol. $\%$ sintering additives are used, secondary phases of $\mathrm{TaB}_{2}$ and/or $\mathrm{NbB}_{2}$ are formed when $\mathrm{B}_{4} \mathrm{C}$ is added and $\mathrm{SiC}$ is formed when $\mathrm{Si}$ is added. These results match what was seen without the addition of GNP.

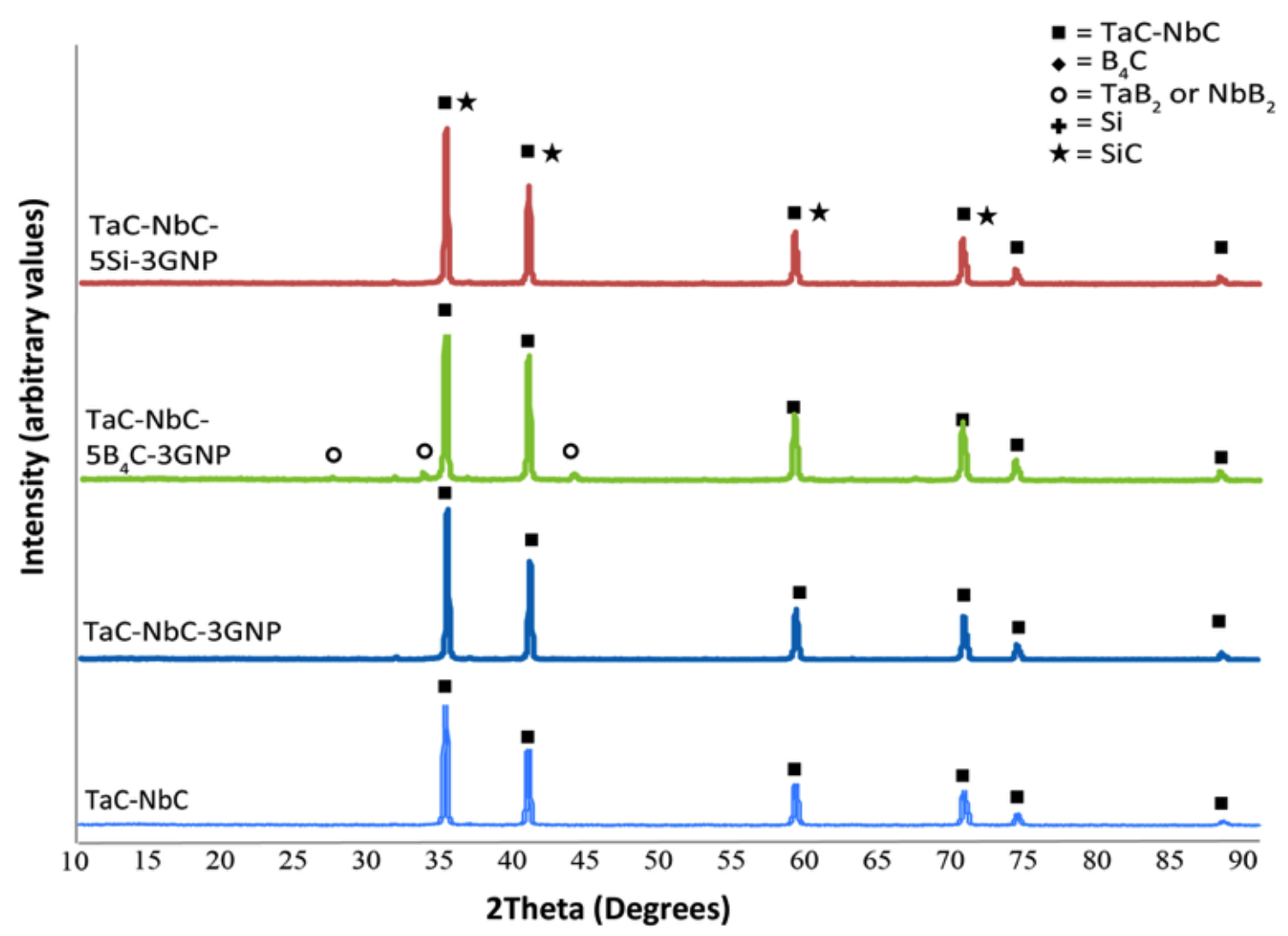

Figure 60: X-ray diffraction patterns for TaC-NbC-3GNP composites with and without sintering additives after spark plasma sintering.

The SiC peaks (JSPDS: 29-1129, 29-1131) are believed to overlap with the TaC-NbC peaks, however due to the small volume percentage of Si added to the composite, they could not be discerned on the XRD plots [76]. This overlap of $\mathrm{SiC}$ has been previously 
reported when adding $\mathrm{Si}$ or $\mathrm{SiC}$ to $\mathrm{TaC}$ composites but only at concentrations greater than 37 vol.\% $[39,69]$.

\subsubsection{Comparison to Compositions without GNP Addition}

In comparing the effect of the various additives on the sintering behavior of the compositions, a correlation was made between the thermal and electrical properties of the phases with the resulting microstructure. When the effect of GNP addition on the TaC$\mathrm{NbC}-5 \mathrm{~B}_{4} \mathrm{C}$ sample was examined, increased grain growth was noticed (Table 13). One possibility for this is that excess carbon by way of GNP addition as well as from the formation of the $(\mathrm{Ta}, \mathrm{Nb}) \mathrm{B}_{2}$ resulting from the reaction $\left[(\mathrm{Ta}, \mathrm{Nb}) \mathrm{C}+\mathrm{B}_{4} \mathrm{C}=>(\mathrm{Ta}, \mathrm{Nb}) \mathrm{B}_{2}+\right.$ $(\mathrm{Ta}, \mathrm{Nb}) \mathrm{C}+\mathrm{C}]$ contributed to excess internal temperature rise and electromigration after $(\mathrm{Ta}, \mathrm{Nb}) \mathrm{B}_{2}$ formation. The GNP and excess carbon increase the electrical conductivity and consequentially leads to an increase in local internal heating by Joule heating.

Table 13: Comparison of microstructures and SPS currents of the various ceramic compositions.

\begin{tabular}{|l|c|c|l|}
\hline \multicolumn{1}{|c|}{ Sample } & Density & $\begin{array}{c}\text { Avg. Grain } \\
\text { Size }(\mu \mathrm{m})\end{array}$ & \multicolumn{1}{c|}{$\begin{array}{c}\text { SPS Current } \\
\text { (lowest [1] to highest [6]) }\end{array}$} \\
\hline TaC-NbC & $86.9 \%$ & 0.78 & 1 \\
\hline TaC-NbC-3GNP & $87.3 \%$ & 0.78 & 4 \\
\hline TaC-NbC-5B 4 C & $99.1 \%$ & 0.83 & 2 \\
\hline TaC-NbC-5B 4 C-3GNP & $98.7 \%$ & 1.53 & 5 until 790 sec then 6 \\
\hline TaC-NbC-5Si & $99.3 \%$ & 2.26 & 3 \\
\hline TaC-NbC-5Si-3GNP & $99.0 \%$ & 1.35 & 6 until 790 sec then 5 \\
\hline
\end{tabular}

When the effect of GNP addition on the TaC-NbC-5Si sample was examined, the grain size decreased with the addition of GNP. One possibility for this occurrence is that with the addition of the GNP, the $\mathrm{Si}$ reacted to form $\mathrm{SiC}$ at an earlier point in the sintering process which resulted in more $(\mathrm{Ta}, \mathrm{Nb}) \mathrm{C}$ grain pinning by the $\mathrm{SiC}$ phase, which 
prevented the grains from growing. This phenomenon has been previously seen by other researchers $[39,46]$. In addition, due to the reaction to form the $\mathrm{SiC}$, there was not as much excess carbon or GNP left in the sample when compared to the samples with $\mathrm{B}_{4} \mathrm{C}$ addition. The resulting decrease in thermal conductivity of the sample would lead to a reduction in excess internal temperatures of the powder compact during sintering which could also result in a reduction in grain growth. Evidence of this can be seen in the plotting of the current output during SPS processing (Figure 61). At about 790 seconds, the current necessary to obtain the same temperature for the TaC-NbC-5B ${ }_{4} \mathrm{C}-3 \mathrm{GNP}$ and TaC-NbC-5Si-3GNP samples flip flop which points to a change in thermal conductivity of the sample.

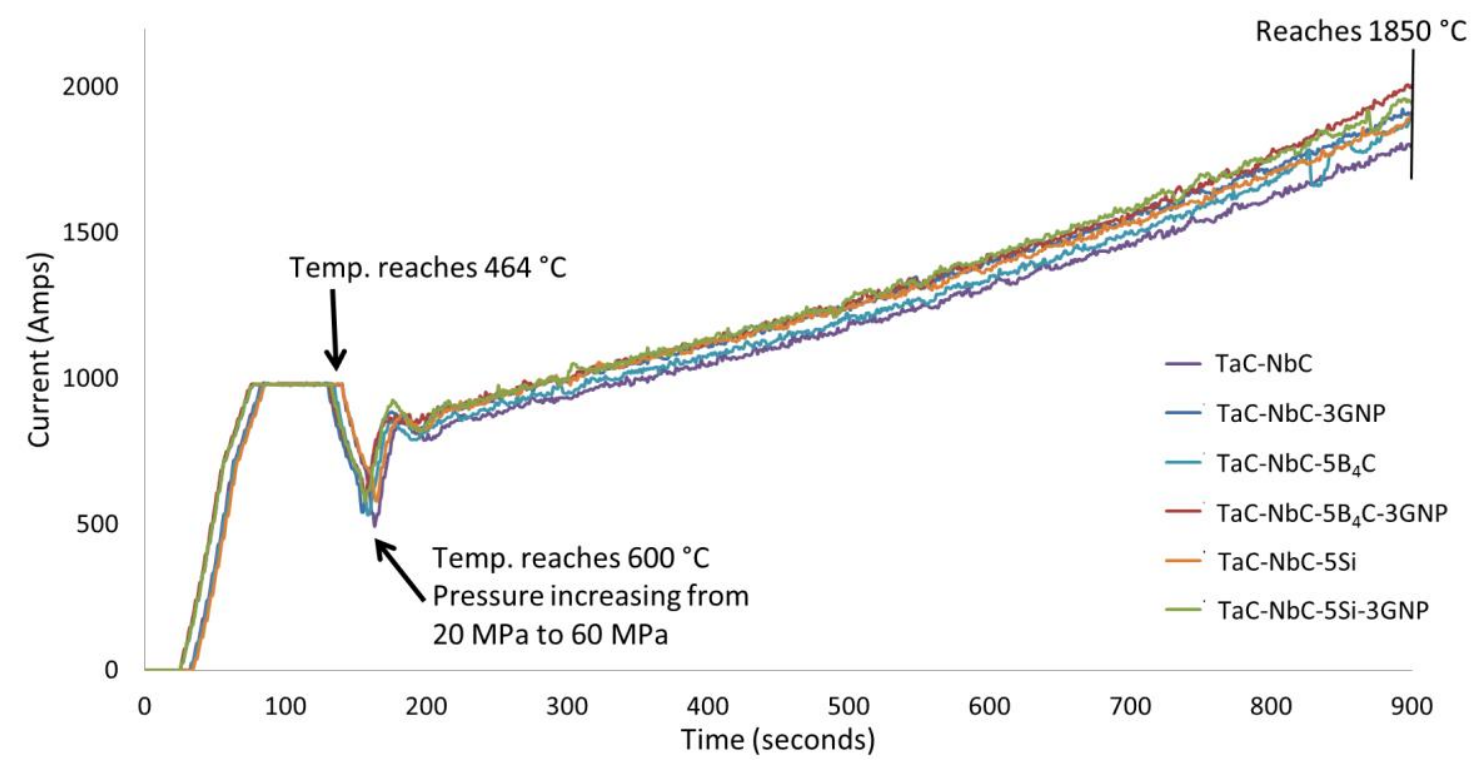

Figure 61: Plots of the current outputs during SPS processing for the various ceramic compositions. 


\subsubsection{Mechanical Properties by Indentation}

As a result of the aligned orientation of the GNP in the TaC-NbC-3GNP and TaCNbC-5B ${ }_{4} \mathrm{C}-3 \mathrm{GNP}$ samples and the alignment of the $\mathrm{SiC}$ phases and smaller occurrences of GNP alignment in the TaC-NbC-5Si-3GNP sample, the mechanical properties are evaluated in both the surface and orthogonal directions shown in Figure 62. By performing indentations in both directions, the anisotropy of the toughening benefits of GNP addition was evaluated.
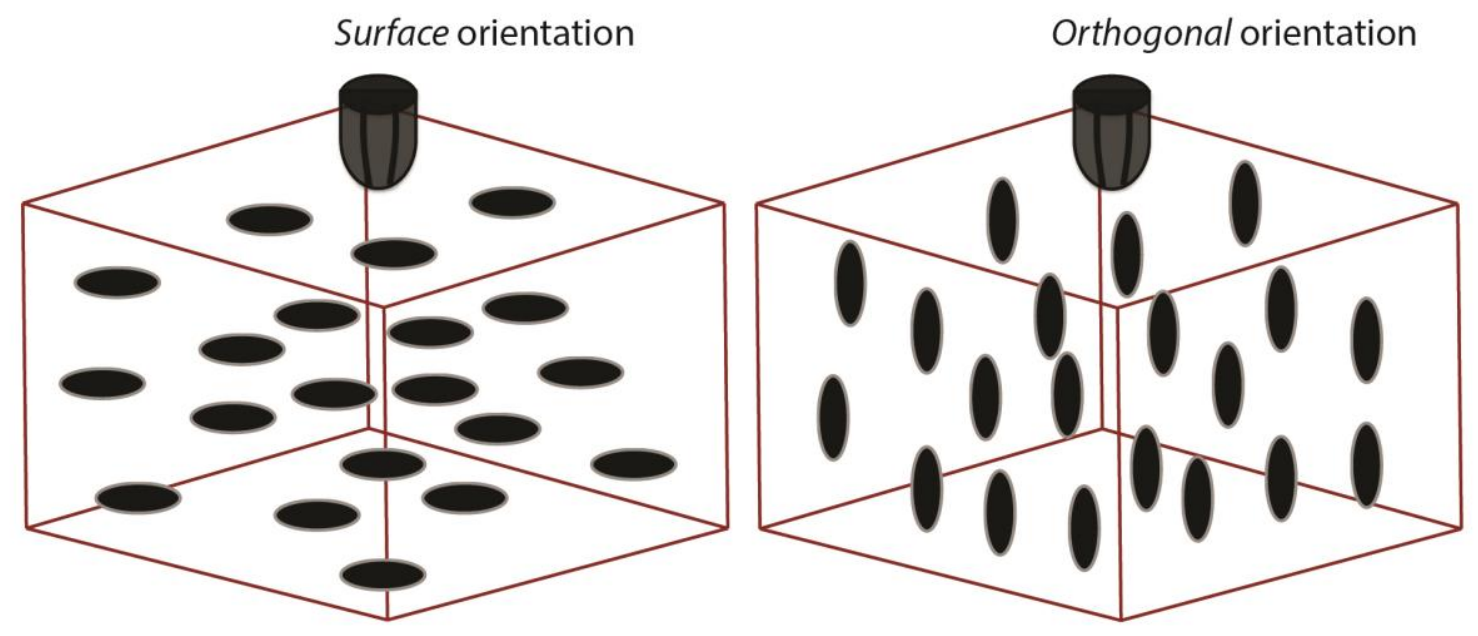

Figure 62: Schematic of indentation directions with respect to the GNP orientation.

Vickers hardness increased with the addition of sintering additives as shown in

Table 14. This increase is mainly attributed to the increase in densification with respect to the sample without sintering additive. With respect to the indentation orientation in relation to the GNP alignment, indentation in the surface direction results in a $15 \%$ and $6 \%$ increase in hardness for the TaC-NbC-3GNP and TaC-NbC-5B4C-3GNP samples, respectively. The sample with $\mathrm{Si}$ addition did not follow the same trend. The hardness value in the orthogonal indentation direction was $10 \%$ greater than in the surface 
direction. This break in the trend is likely due to the formation of $\mathrm{SiC}$ which reacted with the GNP and took its place in the composite.

Table 14: Indentation properties of spark plasma sintered TaC-NbC compacts.

\begin{tabular}{|c|c|c|c|c|}
\hline Sample & $\begin{array}{c}\text { Hardness } \\
\text { (GPa) }\end{array}$ & $\begin{array}{c}\text { Projected } \\
\text { Damaged Area } \\
\left(\mathrm{mm}^{2}\right)\end{array}$ & $\begin{array}{l}\text { Relative } \\
\text { Density }\end{array}$ & $\begin{array}{c}\text { Grain Size } \\
(\mu \mathrm{m})\end{array}$ \\
\hline TaC-NbC & $7.63 \pm 0.47$ & 1.020 & $86.9 \%$ & $0.78 \pm 0.18$ \\
\hline $\begin{array}{c}\text { TaC-NbC-3GNP } \\
\text { Surface }\end{array}$ & $10.44 \pm 0.70$ & 1.068 & \multirow{2}{*}{$87.3 \%$} & \multirow{2}{*}{$0.78 \pm 0.14$} \\
\hline $\begin{array}{c}\text { TaC-NbC-3GNP } \\
\text { Orthogonal }\end{array}$ & $8.96 \pm 0.57$ & 0.794 & & \\
\hline $\begin{array}{c}\text { TaC-NbC-5B }{ }_{4} \mathrm{C}- \\
\text { 3GNP Surface }\end{array}$ & $15.55 \pm 0.89$ & 0.954 & \multirow{2}{*}{$98.7 \%$} & \multirow{2}{*}{$1.53 \pm 0.29$} \\
\hline $\begin{array}{l}\text { TaC-NbC-5B }{ }_{4} \mathrm{C}- \\
\text { 3GNP Orthogonal }\end{array}$ & $14.60 \pm 0.73$ & 0.504 & & \\
\hline $\begin{array}{l}\text { TaC-NbC-5Si- } \\
\text { 3GNP Surface }\end{array}$ & $13.21 \pm 0.70$ & 0.743 & \multirow{2}{*}{$99.0 \%$} & \multirow{2}{*}{$1.35 \pm 0.31$} \\
\hline $\begin{array}{c}\text { TaC-NbC-5Si- } \\
\text { 3GNP Orthogonal }\end{array}$ & $14.61 \pm 0.55$ & 0.379 & & \\
\hline
\end{tabular}

Load-displacement curves from high load instrumented indentation are shown in Figure 63 for indentation on TaC-NbC-5B 4 C-3GNP and Figure 64 for indentation on TaC-NbC-5Si-3GNP. Each figure compares the curves of indentation occurring in the surface and orthogonal indentation orientations. Curves are only being shown for the samples with sintering additive due to the density of the sample without sintering additive being too low for accurate comparison. 
TaC-NbC-5B ${ }_{4} \mathrm{C}-3 \mathrm{GNP}$

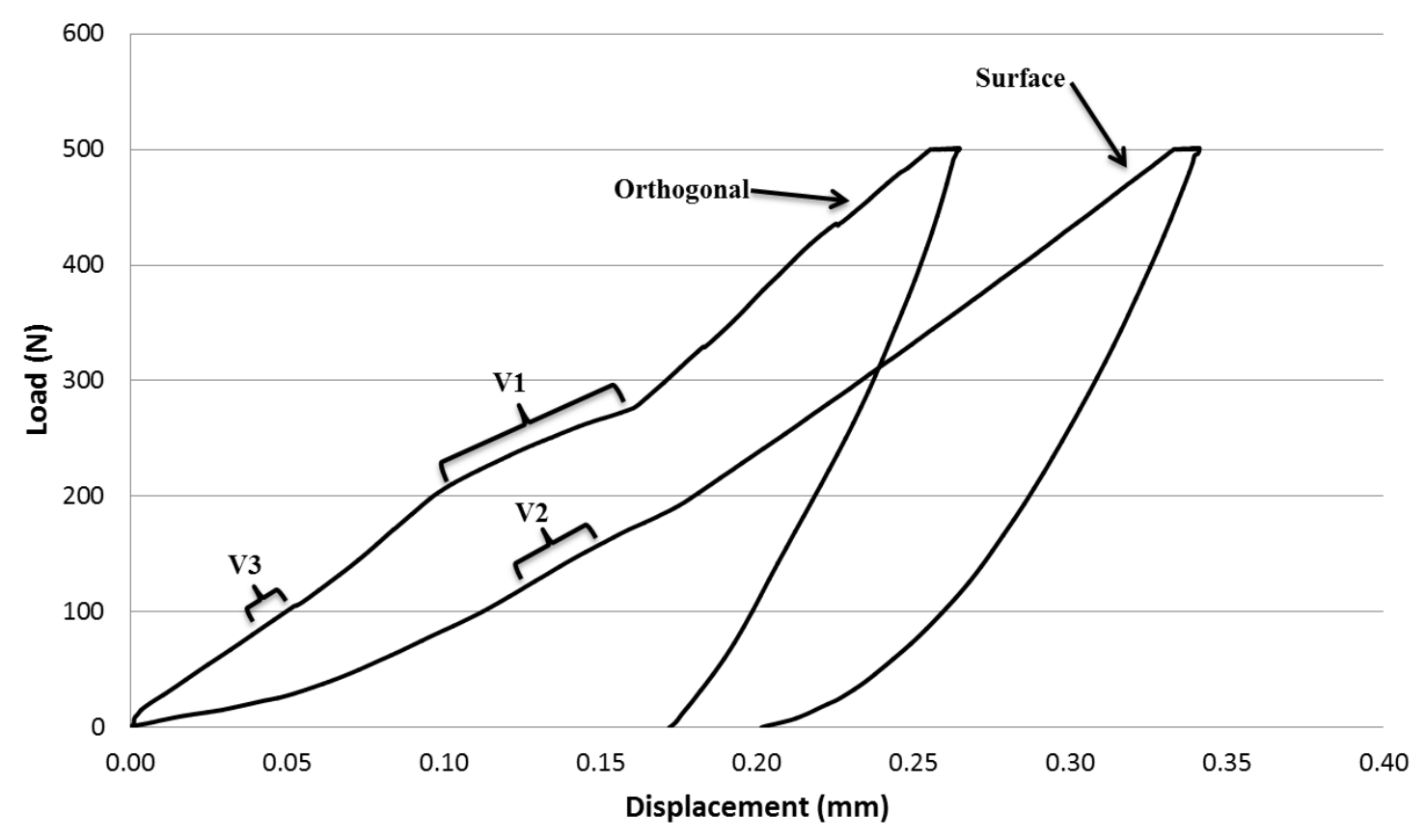

Figure 63: High load instrumented indentation load-displacement curves for the TaC$\mathrm{NbC}-5 \mathrm{~B}_{4} \mathrm{C}-3 \mathrm{GNP}$ composite in both the surface and orthogonal directions. Ranges of supplementary videos marked on curves.

In comparing the indents in respect to indentation direction, it is clear that for the same load, the indentation depth is larger in the surface orientation. Nieto et al. [21] reported that when a $\mathrm{TaC}$ grain is being indented during nanoindentation in a composite without GNP addition, the grain is held tightly by other $\mathrm{TaC}$ grains under and adjacent to it. However, with the addition of GNP, increased displacement can result due to the shearing between graphene sheets that will cause the $\mathrm{TaC}$ grain to slip. When indentation is performed in the surface direction, the orientation of the GNP results in a greater surface area for the $\mathrm{TaC}-\mathrm{NbC}$ grains to interact with leading to increased grain slipping and indentation depth. 
For the sample with $\mathrm{B}_{4} \mathrm{C}$ addition, the depth of penetration was $31 \%$ greater in the surface direction $(0.333 \mathrm{~mm})$ than in the orthogonal direction $(0.254 \mathrm{~mm})$ at the same 500 $\mathrm{N}$ load. In the orthogonal indentation, a change in slope can be seen between displacements of $0.1 \mathrm{~mm}$ and $0.16 \mathrm{~mm}$ (marked as V1 on the figure). Due to these indentations being performed in-situ which allows real-time microscopic viewing, this change in slope is a result of two cracks convening, which causes a fracture to occur on the sample that reduces the resistance to the indentation. Even with this occurrence, the indentation in the orthogonal direction shows a greater resistance to an applied force which is attributed to the indentation occurring on the in-plane orientation of the aligned GNP which results in bending and kinking of the GNP that dissipates force.

For the sample with $\mathrm{Si}$ addition, the depth of penetration was $64 \%$ greater in the surface direction $(0.353 \mathrm{~mm})$ than in the orthogonal direction $(0.215 \mathrm{~mm})$. While the majority of the GNP reacted with the $\mathrm{Si}$ to form $\mathrm{SiC}$, an aligned orientation was still displayed. 
TaC-NbC-5Si-3GNP

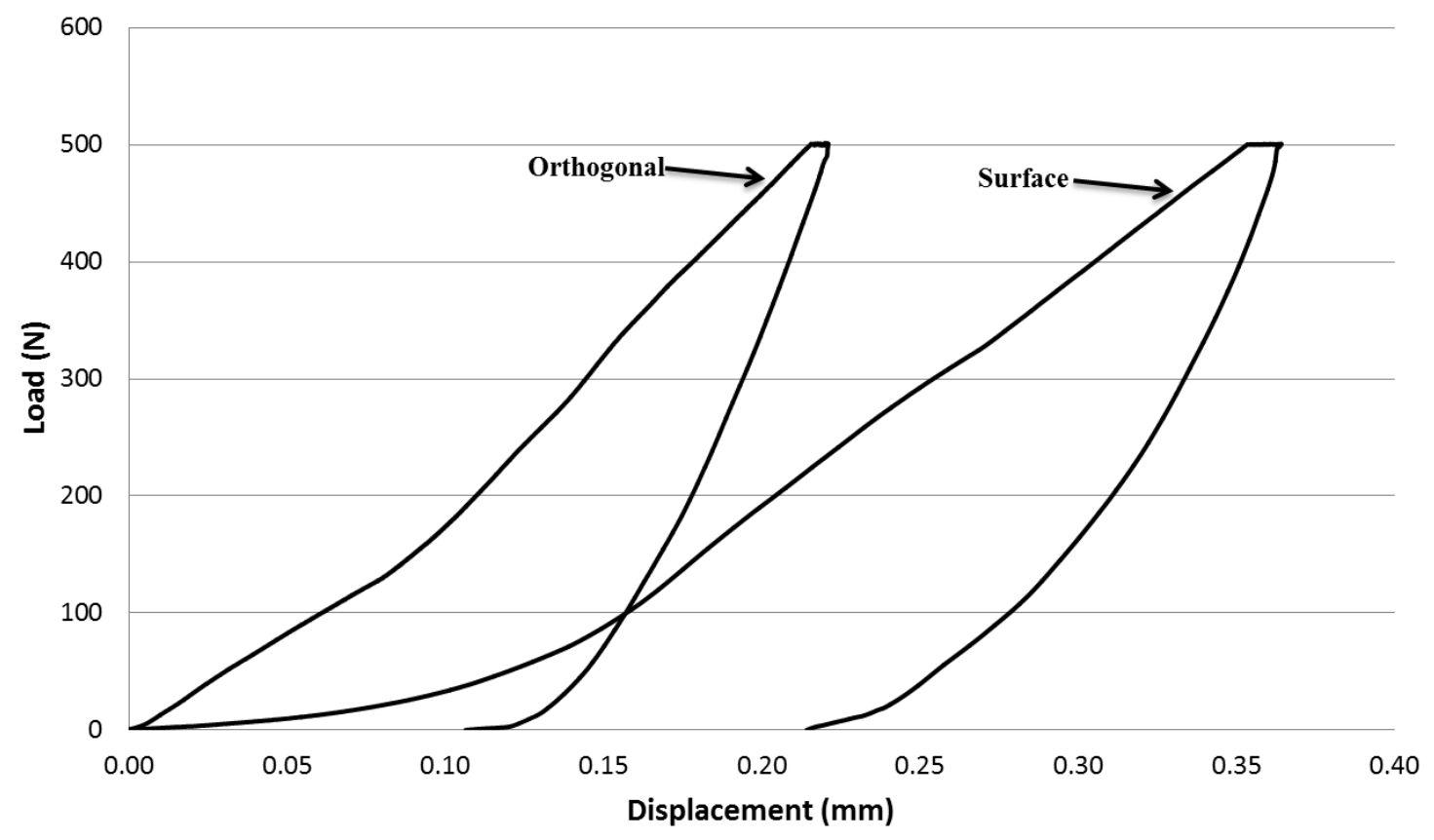

Figure 64: High load instrumented indentation load-displacement curves for the TaCNbC-5Si-3GNP composite in both the surface and orthogonal directions.

The residual damage as a result of high load indentation can be seen in Figure 65 . The impression left by the indentation tip can be clearly seen in Figure 65a, however the cracks that emanate out from the indentation site are not discernable at such a low magnification. Figure $65 \mathrm{~b}$ shows a higher magnification of the area marked in Figure 65a at which cracks can be seen stemming from the indentation site. Due to the inability to accurately perceive the crack lengths of all the cracks that have formed, maps of the residual damaged area for both the surface indentation direction (Figure 66) and the orthogonal indentation direction (Figure 67) were made by tracing the crack lengths originating from the point of indentation and connecting the ends of the cracks to calculate the projected area of residual damage as shown in the schematic of Figure 65c. 

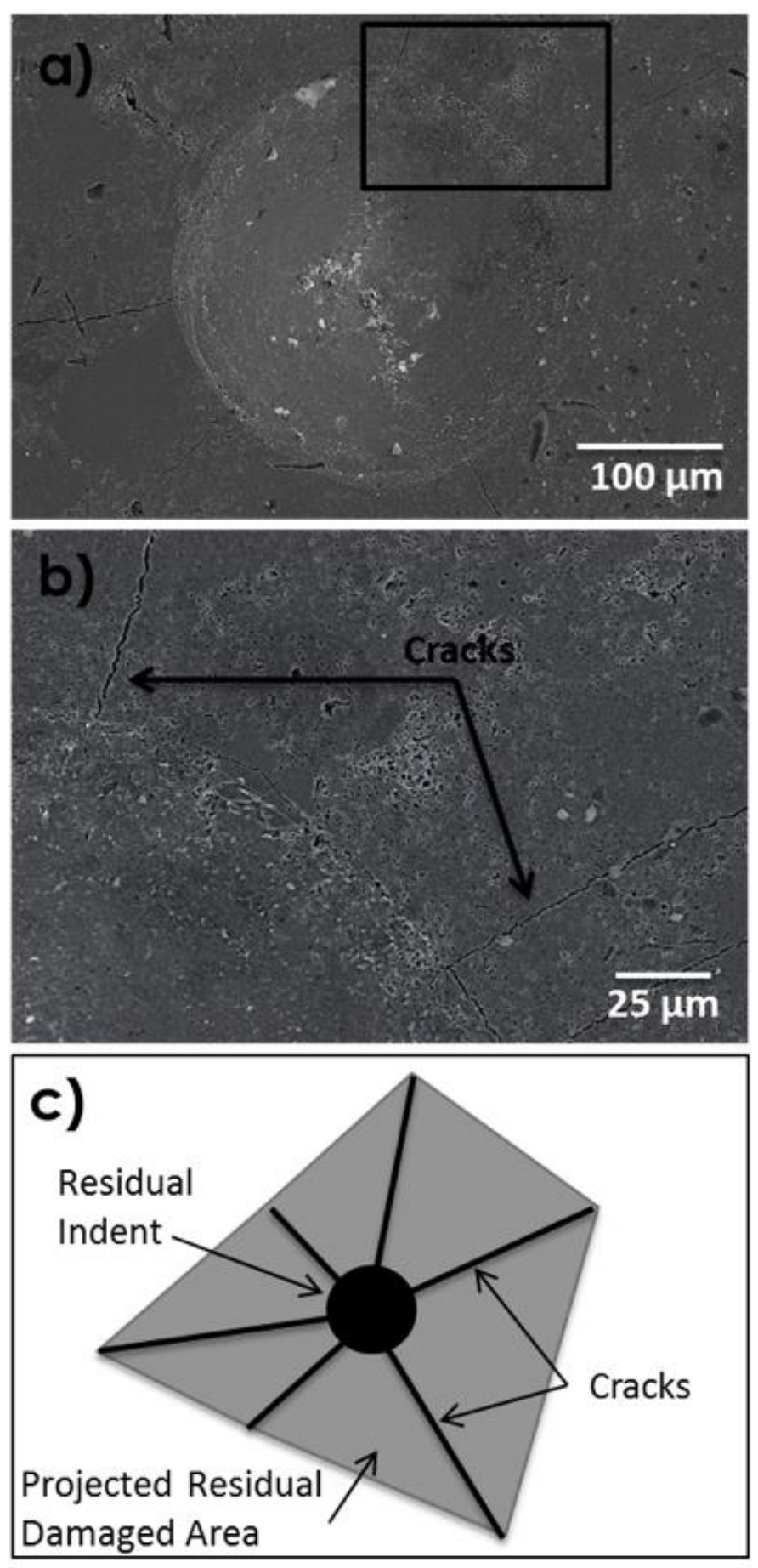

Figure 65: a) SEM image of indentation impression on TaC-NbC-3GNP, b) zoomed in SEM image of area marked on (a) showing cracks protruding from indentation, and c) a schematic of the cracks as a result of indentation with shaded projected residual damaged area. 
When comparing the projected residual damaged areas (stated in Table 14) of the three different compositions, the sample with $\mathrm{Si}$ addition has the least amount of projected residual damage. This is consistent with our findings previously reported which attributed the increased relative toughness to Si having a greater ability to plastically deform compared to ceramics. In addition, the formation of $\mathrm{SiC}$ has added toughening mechanisms. Liu et al. [39] reported pullout of $\mathrm{SiC}$ grains in the fracture surface of a $\mathrm{TaC} / \mathrm{SiC}$ composite which indicates weakened bonding between the $\mathrm{SiC}$ and $\mathrm{TaC}$ grains which in turn favors interface debonding and crack deflection. Padture et al. [77] noted an increase in fracture toughness due to crack bridging in a pressure-less sintered $\mathrm{SiC}$ sample with a heterogeneous grain structure due to $\beta \rightarrow \alpha$ phase transformation.

When analyzing the results with respect to indentation direction, the projected residual damaged area in the surface direction was $34.5 \%$ greater in the TaC-NbC-3GNP sample, $89 \%$ greater in the $\mathrm{TaC}-\mathrm{NbC}-5 \mathrm{~B}_{4} \mathrm{C}-3 \mathrm{GNP}$ sample, and $96 \%$ greater in the $\mathrm{TaC}-$ $\mathrm{NbC}-5 \mathrm{Si}-3 \mathrm{GNP}$ sample when compared to indentation in the orthogonal direction. Therefore, the relative fracture toughness of the composites can be said to be greater in the orthogonal direction compared to in the surface direction. If these materials were being used for thermal protection systems or leading edges, having the GNP aligned perpendicular to the surface would provide greater protection against a strike or impact. The differences in projected residual damaged area with respect to indentation direction further points to the fact that the intrinsic toughening benefits of GNP are anisotropic. 


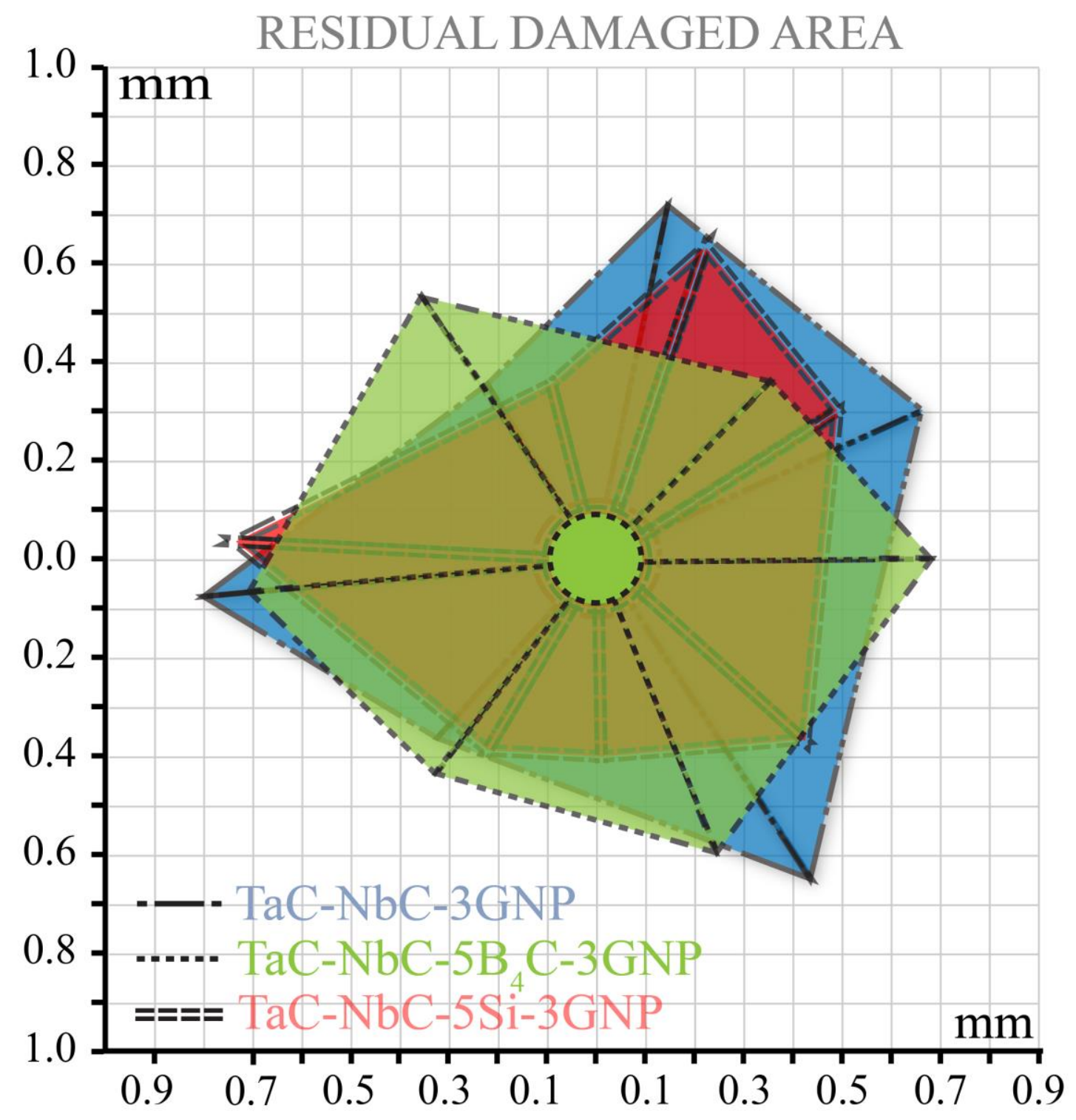

Figure 66: Map of projected residual damage resulting from high load indentation in the surface direction for spark plasma sintered $\mathrm{TaC}-\mathrm{NbC}$ composites. 


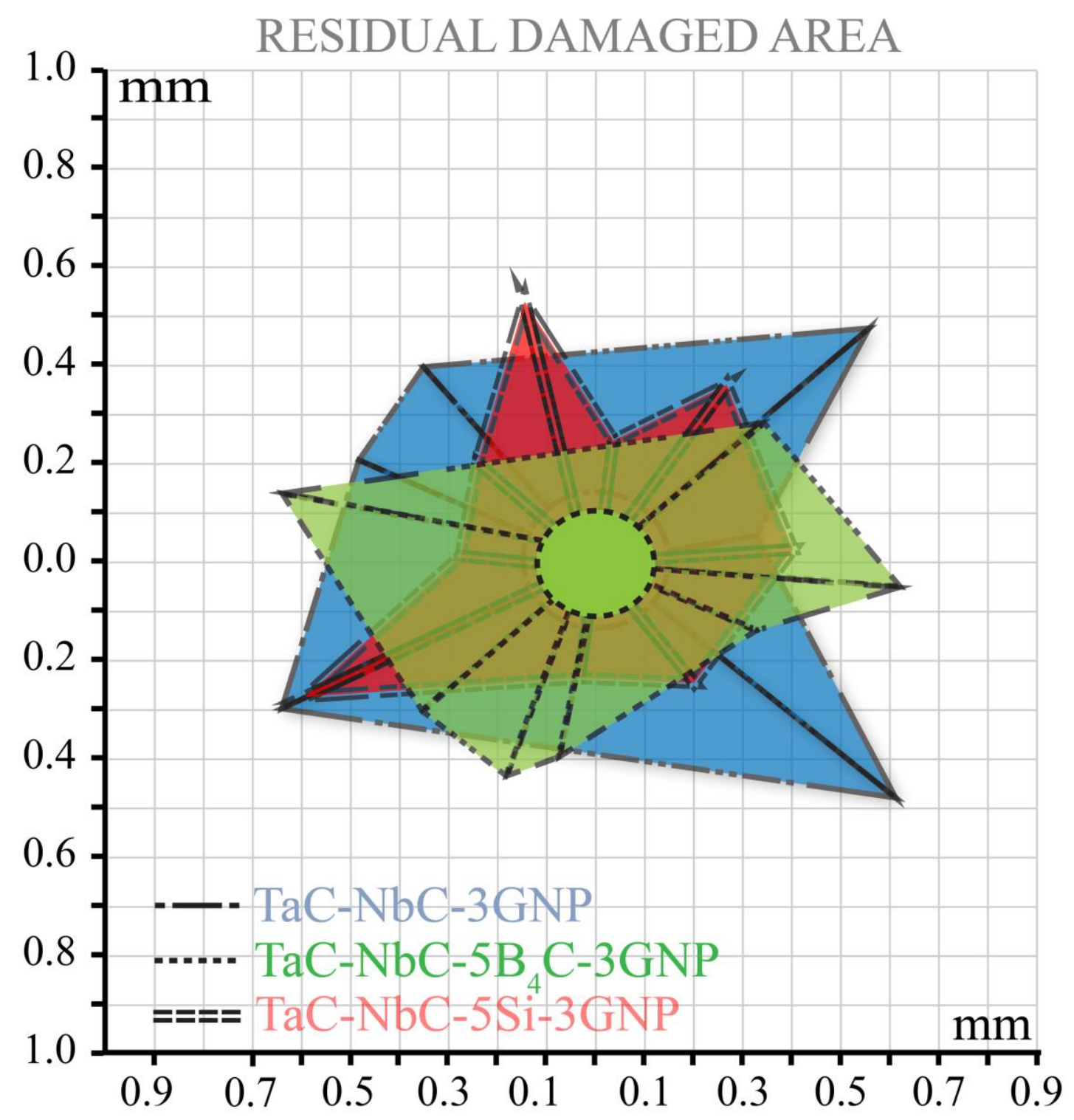

Figure 67: Map of projected residual damage resulting from high load instrumented indentation in the orthogonal direction for spark plasma sintered $\mathrm{TaC}-\mathrm{NbC}$ composites.

An added benefit of these tests being done in situ is the ability to see in real time what deformation mechanisms are occurring. Compared to post-indentation SEM imaging, in situ imaging reveals when these deformation mechanisms take place as well as their frequency. When comparing the indentation videos for the $\mathrm{TaC}-\mathrm{NbC}-5 \mathrm{~B}_{4} \mathrm{C}$ - 
3GNP sample in each indentation orientation, it is evident that the predominant GNP deformation mechanism in the surface orientation was sheet sliding whereas in in the orthogonal indentation direction the predominant mechanism was crack bridging.

Figure 68 shows image stills taken from the real-time video of the indentation in the surface orientation which shows the GNP sliding mechanism. This sliding mechanism was seen during the indentation depth range $(0.123 \mathrm{~mm}$ to $0.154 \mathrm{~mm})$ marked in Figure 63 as V2. As seen in Figure 68a, a small area begins to delaminate from the surface of the ceramic matrix. This area slides up and away from the site where the indentation tip is in contact with the composite. A magnified view showing the visible GNP is shown in Figure 68c. While the sliding is taking place, energy is being dissipated that would otherwise be used in propagating a crack. This sliding is an example of regime 2 toughening. While just one instance of this sheet sliding is shown, it occurred frequently and was the predominant toughening mechanism for indentation performed in the surface orientation. 


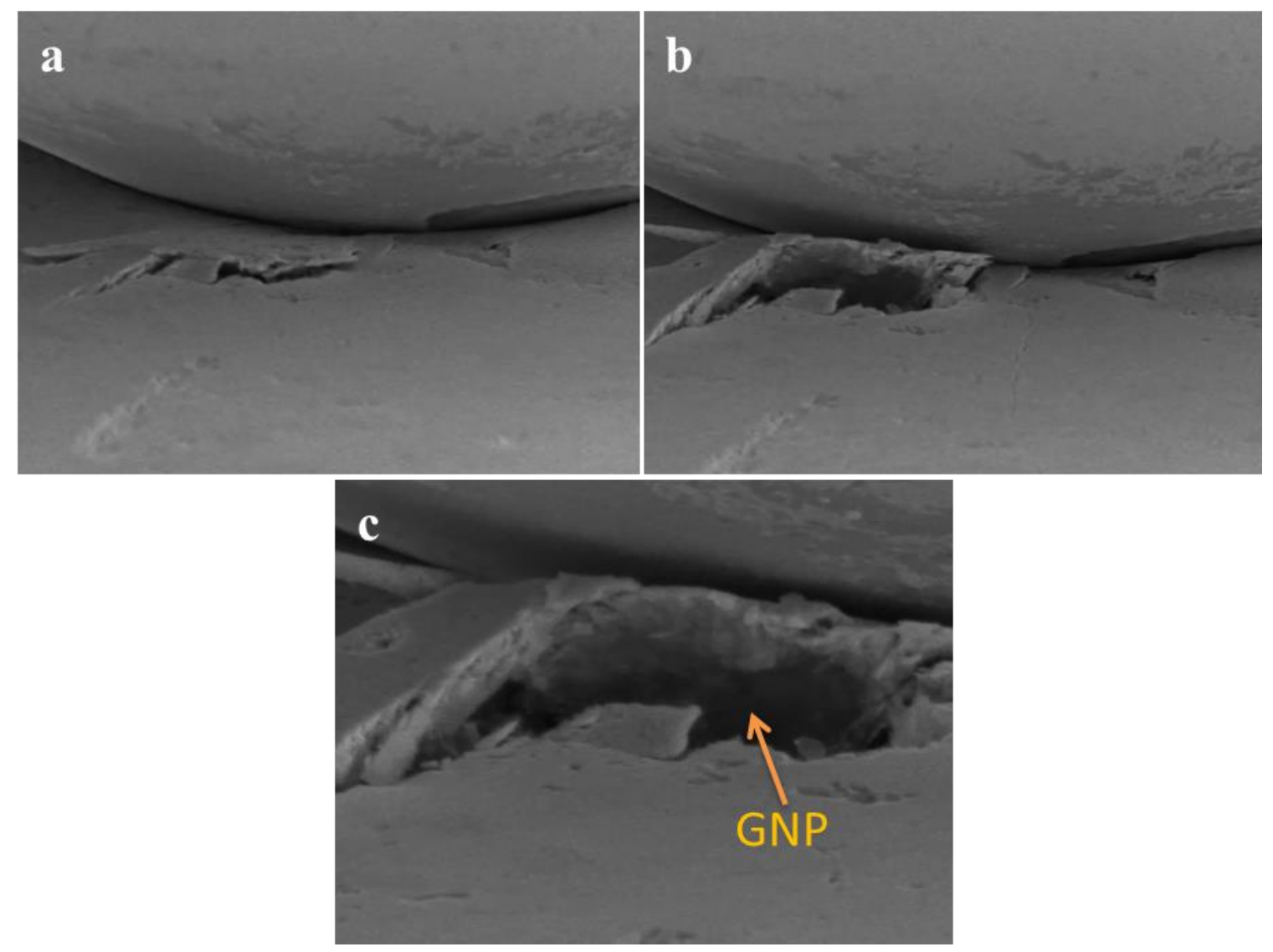

Figure 68: Image stills taken from the real-time indentation video of indentation performed in the surface orientation direction for the TaC-NbC-5B ${ }_{4} \mathrm{C}-3 \mathrm{GNP}$ compact.

At the end of this range, the GNP has completely delaminated from the composite and offers no additional toughening. The decreased slope of the loading curve immediately following provides evidence that the sheet sliding mechanism provided increased load resistance.

Figure 69 shows image stills taken from the real-time video of the indentation in the orthogonal orientation which shows the crack bridging and crack deflection mechanisms. These mechanisms were seen during the indentation depth range $(0.042 \mathrm{~mm}$ to $0.053 \mathrm{~mm}$ ) marked in Figure 63 as V3. In Figure 69a, the GNP that are bridging the crack are marked with the orange arrows while the crack deflection is pointed out with 
the blue arrow. The crack bridging mechanism was most prevalent and acted as regime 3 toughening by suppressing the propagation of the crack up until the point where the crack energy became too great causing the GNP to pull-out as shown by the arrows in Figure 69b. The crack deflection that was seen to occur also acted as regime 3 toughening by diverting the crack from its current path thereby dissipating a greater energy that would otherwise have led to increased crack growth.
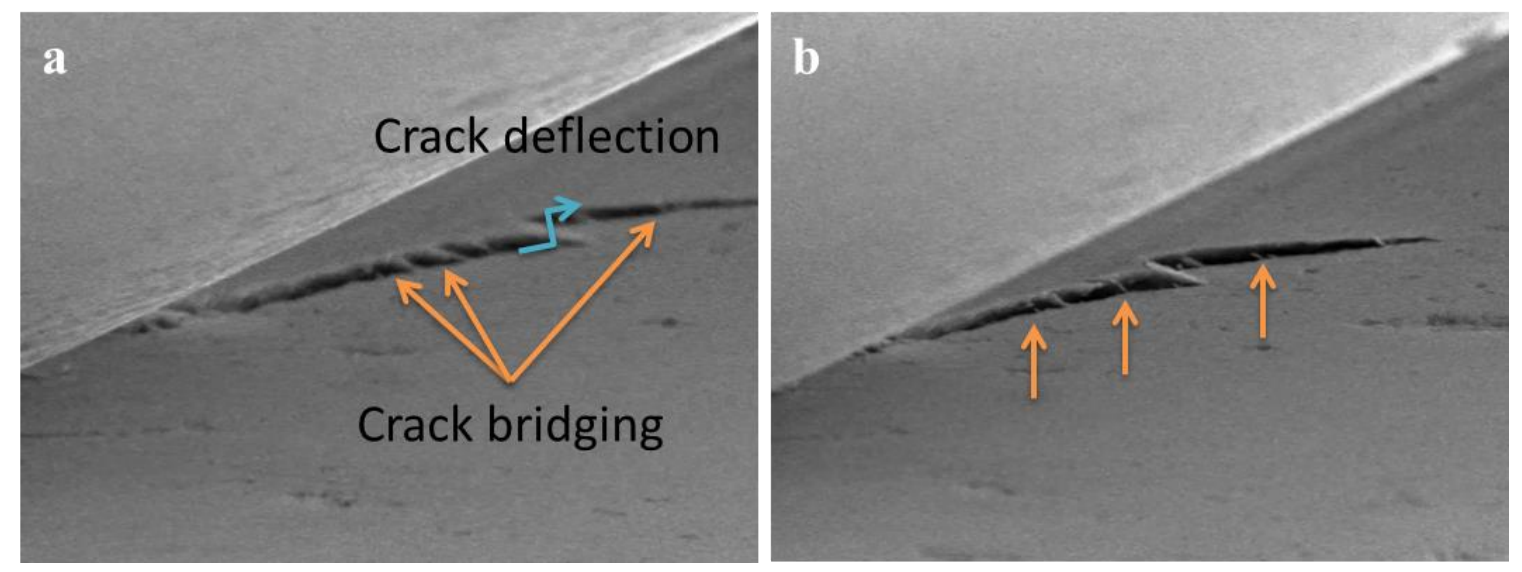

Figure 69: Image stills taken from the real-time indentation video of indentation performed in the orthogonal orientation direction for the TaC-NbC-5B 4 C-3GNP compact.

The analysis of the video for the sample with $\mathrm{Si}$ addition is not being discussed due to the inability to conclude that the toughening is from GNP alone as a result of the added $\mathrm{SiC}$ toughening.

While the in situ videos are beneficial to provide real time mechanism occurrence, post indent imaging provides greater detail. Figure 70 shows post-indentation SEM images of observed intrinsic toughening mechanisms of GNP. When indentation was performed in the orthogonal direction, the predominant observed GNP toughening mechanisms were crack bridging and crack deflection shown in Figure 70a and Figure 
70b, respectively. When indentation was performed in the surface direction, the predominant GNP toughening mechanisms were crack bridging (Figure 70c), crack arrest (Figure 70d), and sheet sliding (Figure 70e). Sheet sliding results from the weak van der Waals forces between graphene layers and therefore dissipates the least amount of indentation energy as was previously reported in section 4.2. Crack arrest occurs when a crack tip comes into contact with GNP and does not have enough energy to deflect around it or through it. This was only seen to occur near the ends of long cracks that did not have enough energy to overcome the obstacle. Crack deflection was observed only in the orthogonal indentation direction. When a crack is deflected it is due to the lowest energy path being between the layers of the GNP. Because of this, crack deflection was only noted when the GNP had a large number of layers as shown in Figure 70b which caused the crack to navigate perpendicular to the crack direction in order to move between layers of graphene. While crack bridging was noted in both the surface and orthogonal orientations, the toughening benefit is greater in the orthogonal direction. As seen in Figure 70a, when a crack is bridged in the orthogonal indentation direction, the crack does not appear to penetrate through the GNP nor does it deviate around the GNP. Instead the crack must propagate below the GNP which changes from a conventional two-dimensional space to force the crack to propagate in three-dimensional space. This occurrence has been previously seen by Walker et al. [60] as well. When a crack is bridged in the surface orientation (Figure 70c), the toughening benefit is much less due to the crack being able to propagate under the thin GNP with little energy dissipation. 

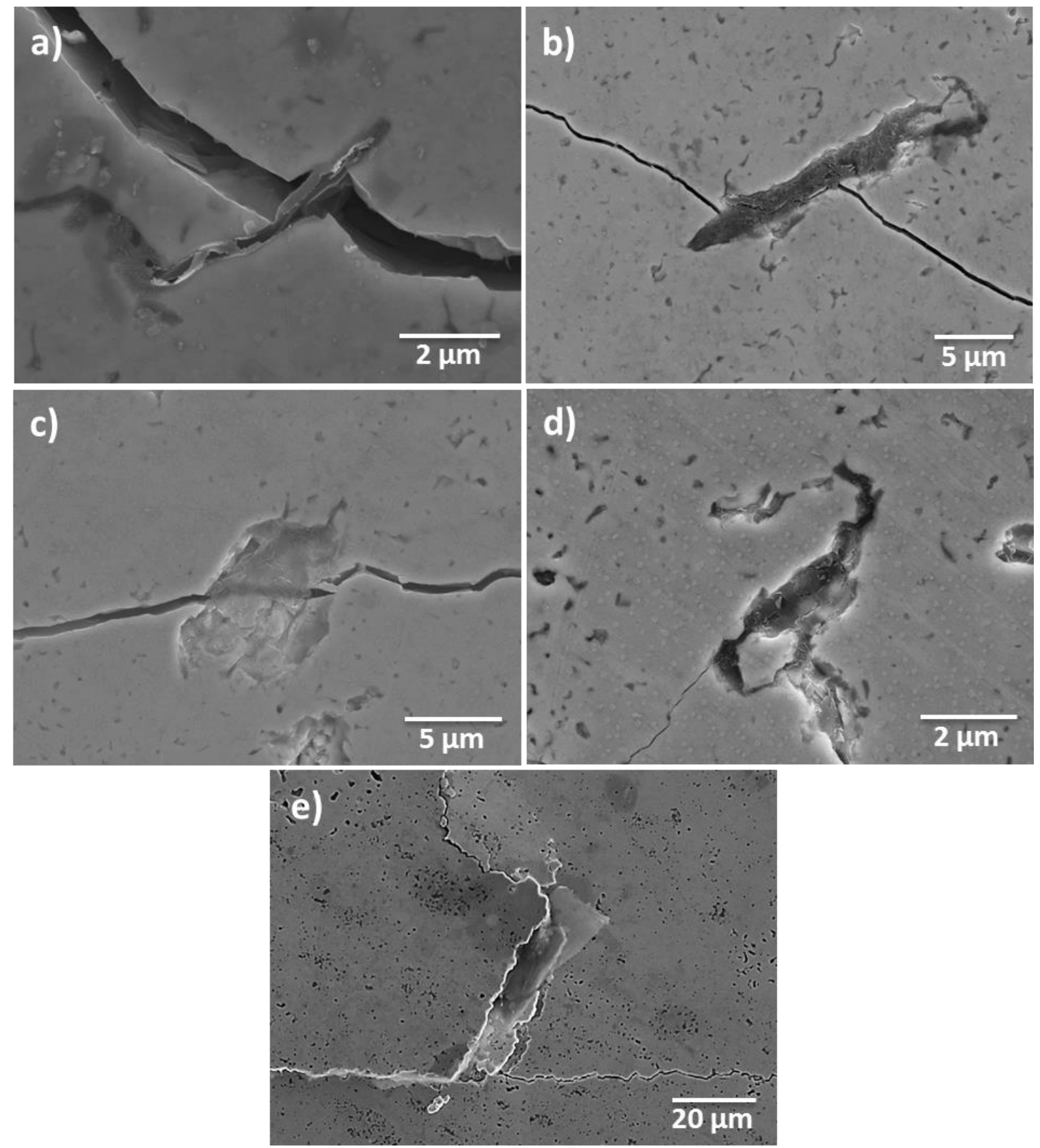

Figure 70: SEM images of intrinsic toughening mechanisms of GNP: a) crack bridging in the orthogonal direction, b) crack deflection, c) crack bridging in the surface direction, d) crack arrest, and e) sheet sliding. 


\subsubsection{Comparison to Compositions without GNP Reinforcement}

Without GNP addition, the projected residual damaged areas of TaC-NbC-5B ${ }_{4} \mathrm{C}$ and TaC-NbC-5Si were $1.025 \mathrm{~mm}^{2}$ and $0.555 \mathrm{~mm}^{2}$, respectively. With the addition of GNP, the damaged area for the sample with $\mathrm{B}_{4} \mathrm{C}$ addition was reduced by $7 \%$ when indenting in the surface direction and reduced by $51 \%$ when indentation was performed in the orthogonal direction. These results indicate an increase in toughness of the GNP reinforced composite. In comparing the TaC-NbC-5Si samples with and without GNP addition, the projected damaged area of the GNP reinforced sample is $33 \%$ greater in the surface indentation orientation but $32 \%$ less in the orthogonal indentation direction compared to the damaged area in the sample without GNP addition. These results point to the fact that the anisotropic toughening of the reinforcement phase is very important. In the $\mathrm{TaC}-\mathrm{NbC}-5 \mathrm{Si}$ sample without GNP addition, the formed SiC phase was randomly oriented throughout the matrix. In the sample with GNP addition, the formed $\mathrm{SiC}$ had an aligned orientation as a result of reacting with the already aligned GNP. The damaged area of the randomly oriented reinforcement sample being right in the middle of the damaged area of the surface and orthogonally oriented reinforcement provides additional evidence that the toughening is highly anisotropic. This outcome also supports the finding that the formation of $\mathrm{SiC}$ acts as a primary reinforcement phase to the composite and that there is anisotropy in the SiC toughening mechanisms (crack bridging and crack deflection) as seen before in $\mathrm{SiC}$ platelet reinforced ceramic composites $[62,72,77]$. 


\subsubsection{Conclusions with Respect to GNP Reinforcement of the Ceramic Composite}

The toughening benefits of adding GNP in the TaC-NbC composite depend on the orientation of the GNP throughout the matrix. Due to the aligned orientation of the GNP as a result of SPS processing, the dominant anisotropic intrinsic toughening mechanisms were able to be analyzed. When indentation was performed on the surface, the dominant toughening mechanisms were sheet sliding, crack bridging, and crack arrest. In the orthogonal orientation, the predominant toughening mechanisms were crack bridging and crack deflection. The relative fracture toughness of the composites is greatest in the orthogonal direction. This result is due to the projected residual damaged area after indentation in the surface direction being $89 \%$ greater in the $\mathrm{TaC}-\mathrm{NbC}-5 \mathrm{~B}_{4} \mathrm{C}-3 \mathrm{GNP}$ sample, and $96 \%$ greater in the $\mathrm{TaC}-\mathrm{NbC}-5 \mathrm{Si}-3 \mathrm{GNP}$ sample when compared to indentation in the orthogonal direction.

\subsection{Compression Testing and Interfacial Stresses}

Diametral compression testing was performed to gain a better understanding of the bulk strength of the sintered pellets. The diametral compression test was chosen due to its ability to test the cylindrical shaped sintered samples without having to first machine them to a desired shape. This test makes the assumption that the maximum tensile principal stress is responsible for the failure of the sample and therefore the tensile strength of the material can be calculated. Table 15 lists the results of the diametral compression tests including the tensile fracture strength $\left(\sigma_{\mathrm{f}}\right)$ and the fracture energy. 
Table 15: Fracture strength and energy results from diametral compression testing.

\begin{tabular}{|c|c|c|c|c|c|}
\hline Sample & $\begin{array}{c}\text { Relative } \\
\text { Density }\end{array}$ & $\begin{array}{c}\text { Max Load } \\
\mathbf{( k N )}\end{array}$ & $\begin{array}{c}\text { Tensile } \\
\text { Fracture } \\
\text { Strength } \\
\text { (MPa) }\end{array}$ & $\begin{array}{c}\text { Fracture } \\
\text { Energy (J) }\end{array}$ & $\begin{array}{c}\text { Normalized } \\
\text { Energy }\end{array}$ \\
\hline TaC-NbC-3GNP & 87.3 & 14.172 & 129.8 & 4.59 & 1 \\
\hline TaC-NbC-5B4C- & 98.7 & 16.377 & 150.0 & 5.90 & 1.29 \\
\hline 3GNP & 99.0 & 23.200 & 212.5 & 12.37 & 2.7 \\
\hline 3aC-NbC-5Si- & & & & & \\
\hline
\end{tabular}

The fracture energies, calculated by the area under the loading curves (Figure 71), are specific to our testing conditions and therefore it is normalized with respect to the sample without sintering additive for a more straightforward comparison between them.

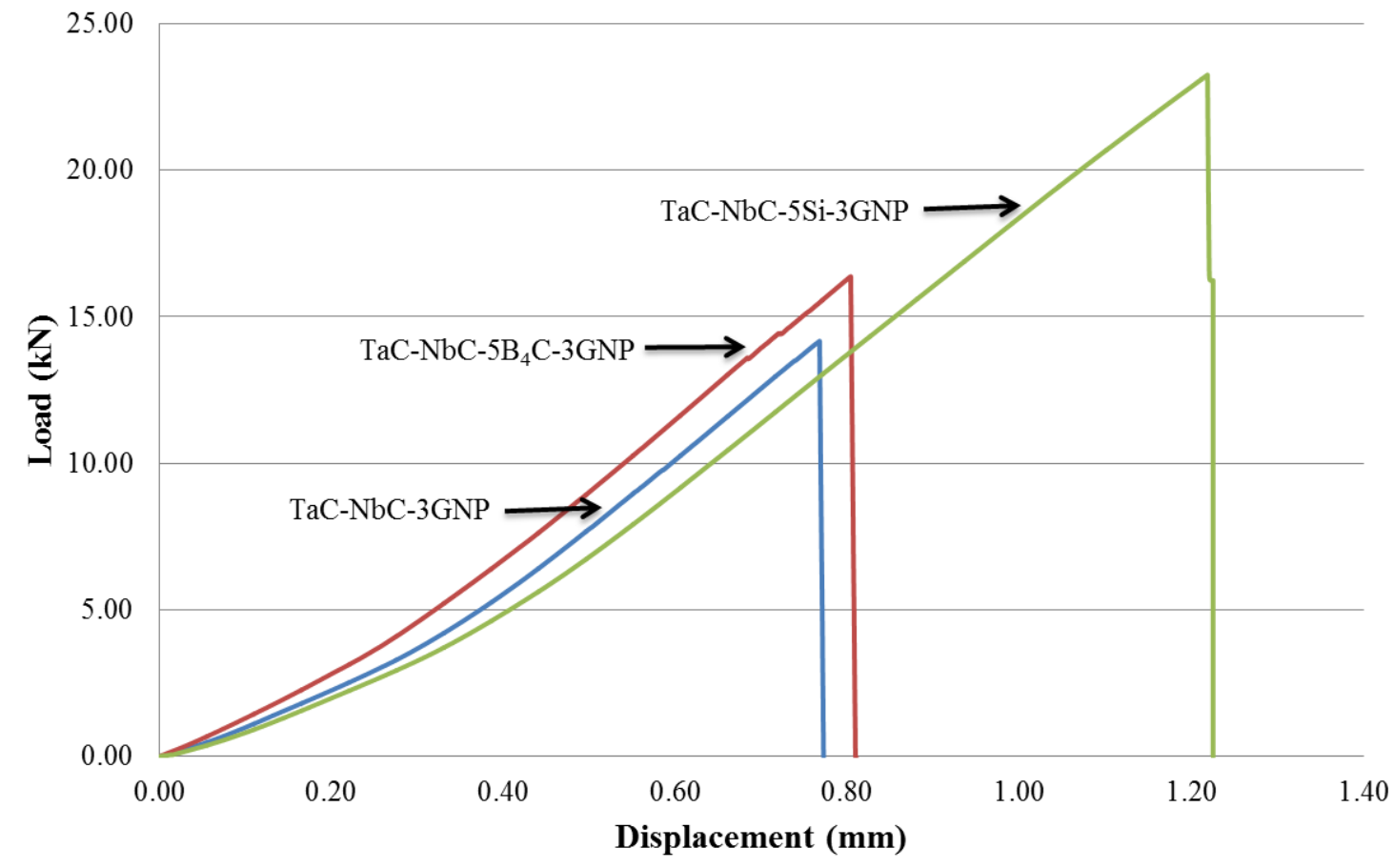

Figure 71: Load-displacement curves resulting from diametral compression testing. 
The sample with $\mathrm{B}_{4} \mathrm{C}$ as the sintering additive resulted in a $16 \%$ increase in fracture strength over the sample without sintering additive. The sample with the greatest fracture strength (TaC-NbC-5Si-3GNP) had an increase in fracture strength of $64 \%$ over the sample without sintering additive. These values follow the same trend as the values we obtained for projected residual damaged area due to indentation and further confirm their validity.

After compression testing, the fracture surfaces of the samples were viewed under SEM to provide insight to the manner of fracture as well as the interfaces between the matrix grains and the GNP reinforcement. When fracture occurred in the sample without sintering additive, it was evident from the GNP sticking out from the surface (Figure 72a and Figure 72b), that debonding between the matrix $\mathrm{TaC}-\mathrm{NbC}$ grains and the GNP occurred on one side (presumably the side with less surface area with respect to the crack location). This debonding between the GNP and the matrix grains is further evident in Figure 72c which shows a void where a GNP debonded and pulled out from the TaC$\mathrm{NbC}$ matrix. Due to the density of this sample only being $87.3 \%$, there is less surface contact between the GNP and the $\mathrm{TaC}-\mathrm{NbC}$ grains which results in this interfacial debonding occurrence. While the addition of the GNP still increases the strength of the composite up to the point where interfacial debonding occurs, the fracture surfaces also showed that the intrinsic toughening mechanism of GNP pull-out occurred. Figure 72d shows a GNP that has increased its surface area with the matrix grains by kinking and wrapping to conform to the $\mathrm{TaC}-\mathrm{NbC}$ grains for stronger bonding. Not only does this occurrence lead to stronger bonding but it also leads to increased load transfer efficiency 
between the matrix and GNP. In this case, it is clearly evident that pull-out occurred between the GNP layers (marked with arrows in the figure) which increases the strength of the composite by dissipating energy that would otherwise propagate the crack.
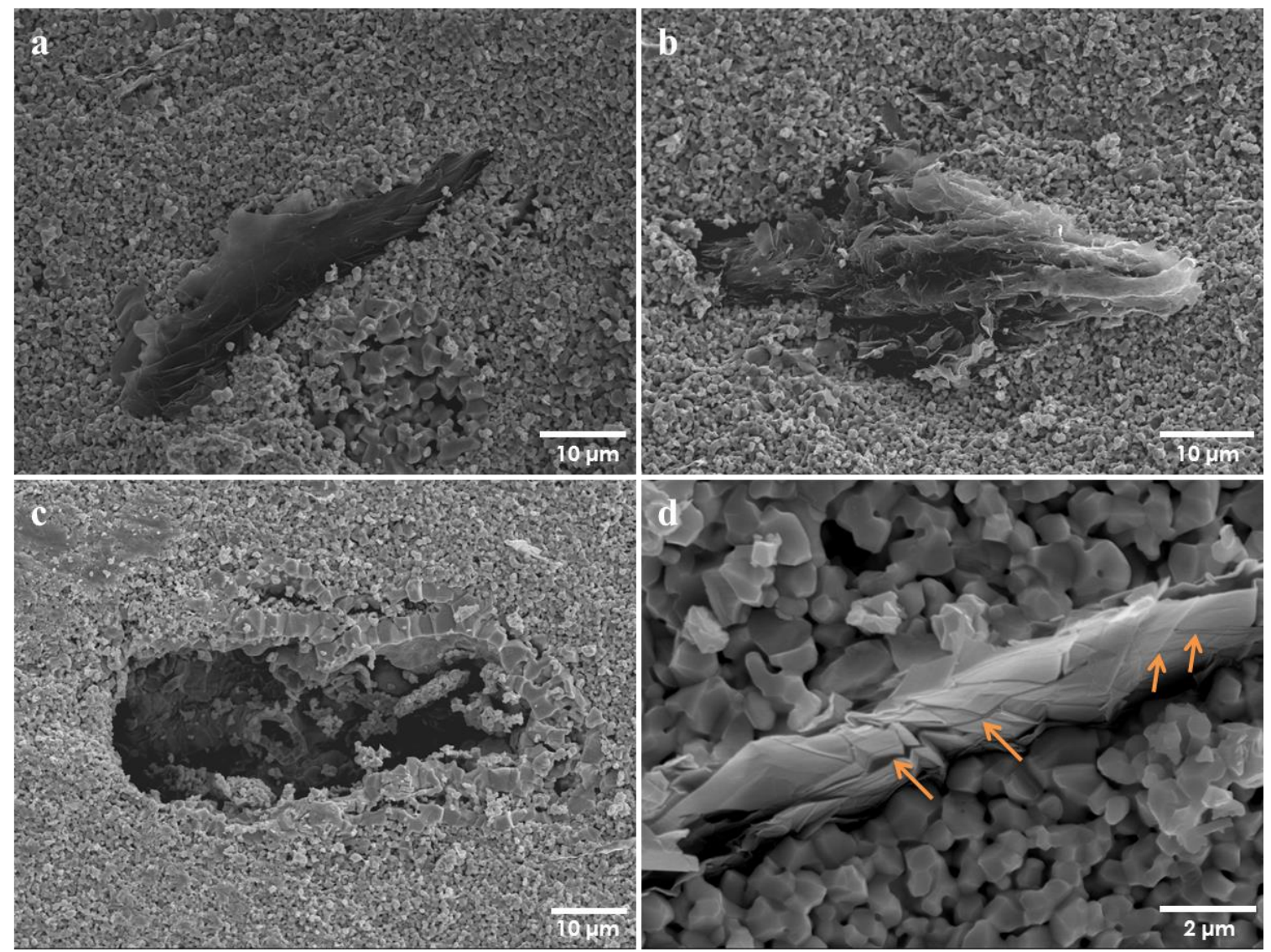

Figure 72: Post compression test SEM imaging of the fracture surface of TaC-NbC3 GNP.

The fracture surface of the sample with $\mathrm{B}_{4} \mathrm{C}$ addition is shown in Figure 73. As in the sample without sintering additive, GNP are seen sticking out from the fracture surface (Figure 73a). Although the same mechanism is seen occurring, the increased density of this sample (98.7\% compared to $87.3 \%$ ) resulted in greater bonding between the GNP and the ceramic matrix grains. Evidence of greater bonding was seen as more instances of pull-out were observed and there were no visible voids (as is the case in Figure 72c) 
where the GNP completely debonded from the ceramic matrix on one side of the fracture. In addition to the GNP mechanisms that were observed, areas of secondary phase formation of $\mathrm{TaB}_{2}$ and/or $\mathrm{NbB}_{2}$ were observed on the fracture surface as well (Figure 73b). These locations seemed to be areas where fracture occurred more easily. This is likely due to the increased hardness of the secondary phase (22.6 and $23.2 \mathrm{GPa}$ for $\mathrm{TaB}_{2}$ and $\mathrm{NbB}_{2}$, respectively versus 16.7 and $19.6 \mathrm{GPa}$ for $\mathrm{TaC}$ and $\mathrm{NbC}$, respectively) that possibly leads to more brittle fracture.
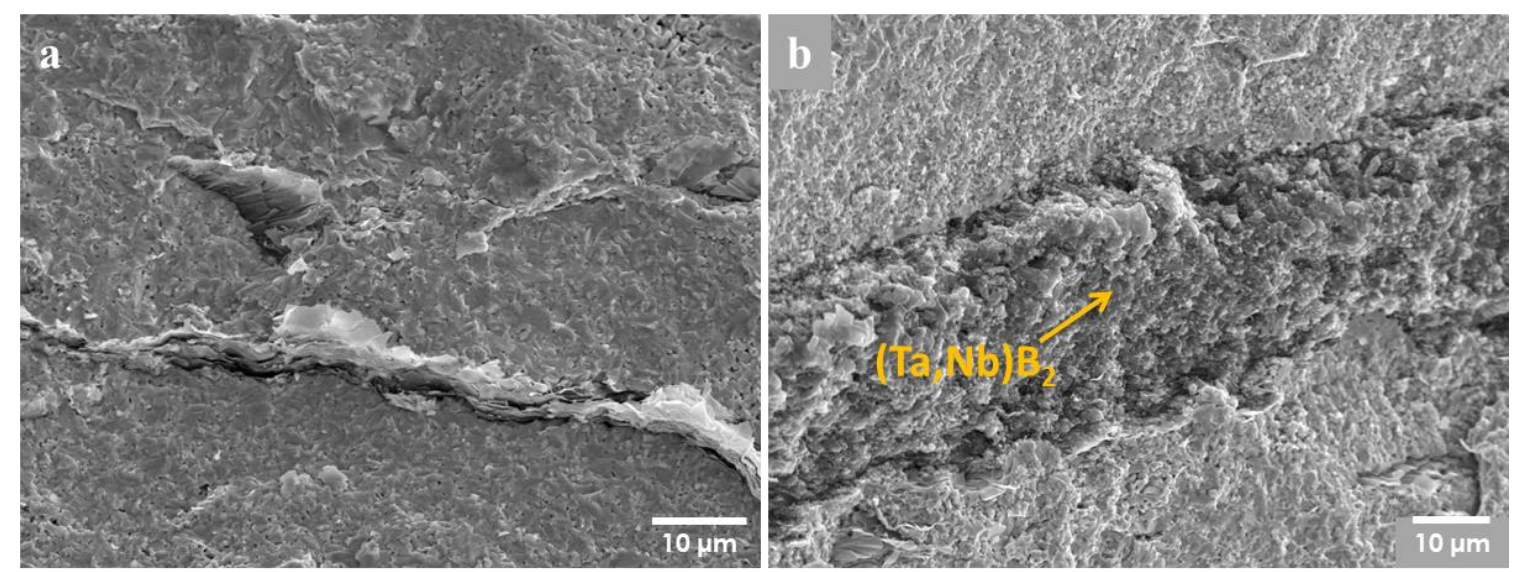

Figure 73: Post compression test SEM imaging of the fracture surface of TaC-NbC$5 \mathrm{~B}_{4} \mathrm{C}-3 \mathrm{GNP}$.

The sample with silicon addition was different from the others. Areas on the fracture surface (Figure 74) that showed GNP sticking out were surrounded by a secondary phase that was confirmed to be SiC by EDS (see appendix 2). As previously stated, the silicon reacted with the GNP to form the $\mathrm{SiC}$ phase. The $\mathrm{SiC}$ phase acts as a reinforcement to the composite in addition to the GNP, which is clearly evident in the increased strength of the sample compared to the other two. 

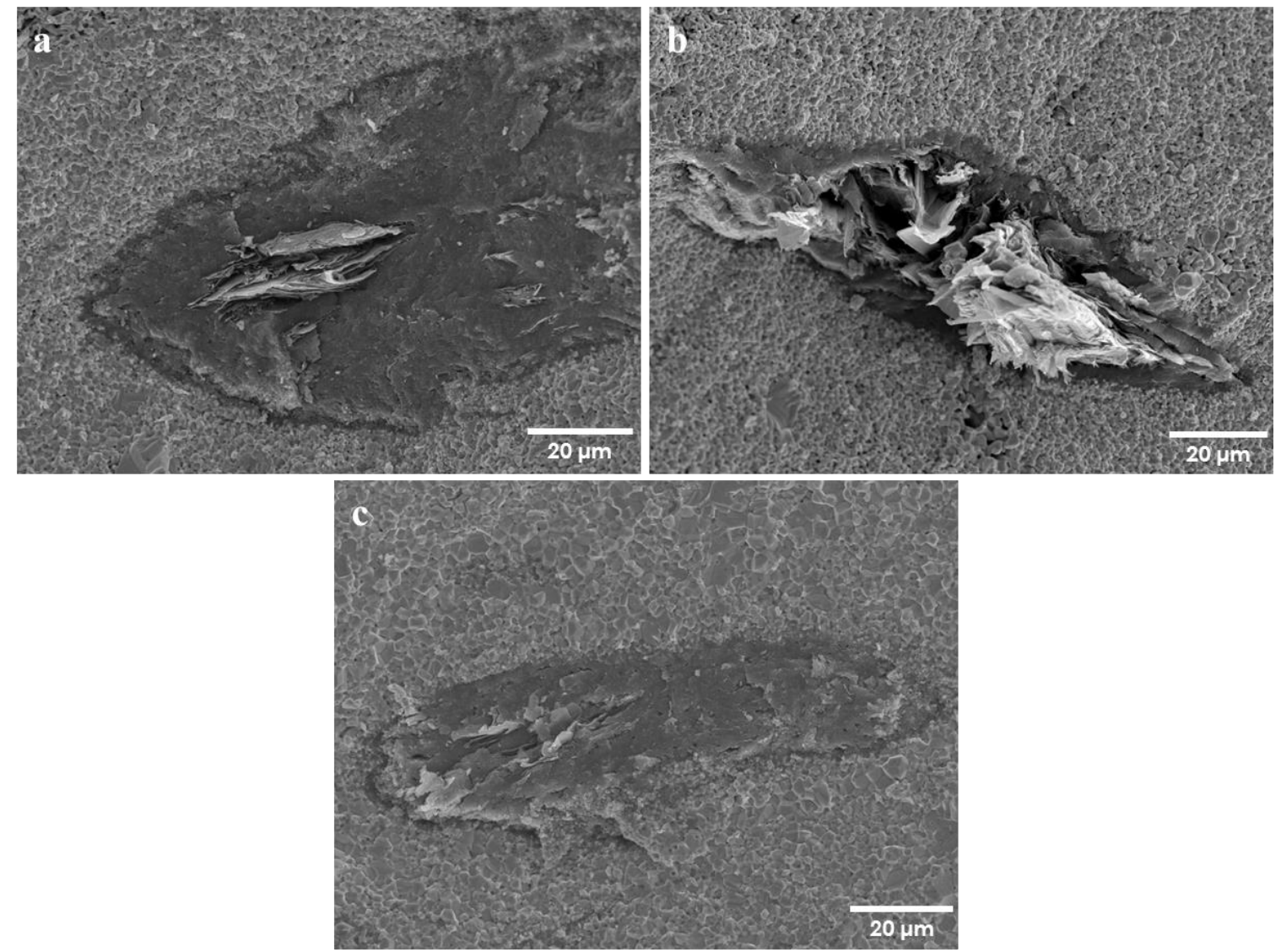

Figure 74: Post compression test SEM imaging of the fracture surface of TaC-NbC-5Si3GNP.

Because the toughening benefit of adding GNP to the ceramic matrix is related to how stress can transfer through the interface between the two, investigation into the interfacial stress is beneficial to understanding how failure will occur at the interface. Using stress transfer equations for a platelet geometry (Figure 75) and making the assumptions that the GNP are all perfectly aligned in the ceramic matrix and that the stress transfer takes place through the interface, the following equations are formed for the interfacial shear stress $(\tau)$ and the axial stress $(\sigma)$ at the GNP-ceramic matrix interface [19]: 


$$
\begin{gathered}
\tau=a \varphi \psi \sigma_{0}\left\{\frac{\exp (\varphi y)-\exp (-\varphi y)}{\exp (\varphi t)+\exp (-\varphi t)}\right\} \\
\sigma=\psi \sigma_{0}\left\{1-\frac{\exp (\varphi y)+\exp (-\varphi y)}{\exp (\varphi t)+\exp (-\varphi t)}\right\}
\end{gathered}
$$

where

$$
\begin{gathered}
\varphi=\frac{1}{b-a}\left\{\frac{3\left(a E_{p}+(b-a) E_{m}\right)}{2 a\left(1+v_{m}\right) E_{p}}\right\}^{1 / 2} \\
\psi=\frac{b E_{p}}{a E_{p}+(b-a) E_{m}}
\end{gathered}
$$

where $E_{m}$ is the elastic modulus of the ceramic, $v_{m}$ is the Poisson's ratio of the ceramic, $\mathrm{E}_{\mathrm{p}}$ is the elastic modulus of the GNP, and $\mathrm{y}$ is the distance from the center of the GNP to its end. Also, the values of $a / b$ and $t / u$ are related to the volume fraction of the added GNP and can be calculated assuming:

$$
\frac{a}{b}=\frac{t}{u}=\frac{1}{m} ; f=\left(\frac{a}{b}\right)\left(\frac{t}{u}\right)^{2}=\left(\frac{1}{m}\right)^{3}
$$

The volume fraction of GNP addition to the composite is estimated using:

$$
f=\frac{\rho_{c}}{\rho_{G}} w_{G} ; \rho_{c}=f \rho_{G}+(1-\Phi-f) \rho_{m}
$$

where $\rho_{\mathrm{c}}$ and $\rho_{\mathrm{m}}$ are the density of the GNP/ceramic composite and the ceramic composite, respectively, $\Phi$ is the volume fraction of porosity and $\mathrm{w}_{\mathrm{G}}$ is the weight fraction of the added GNP. 


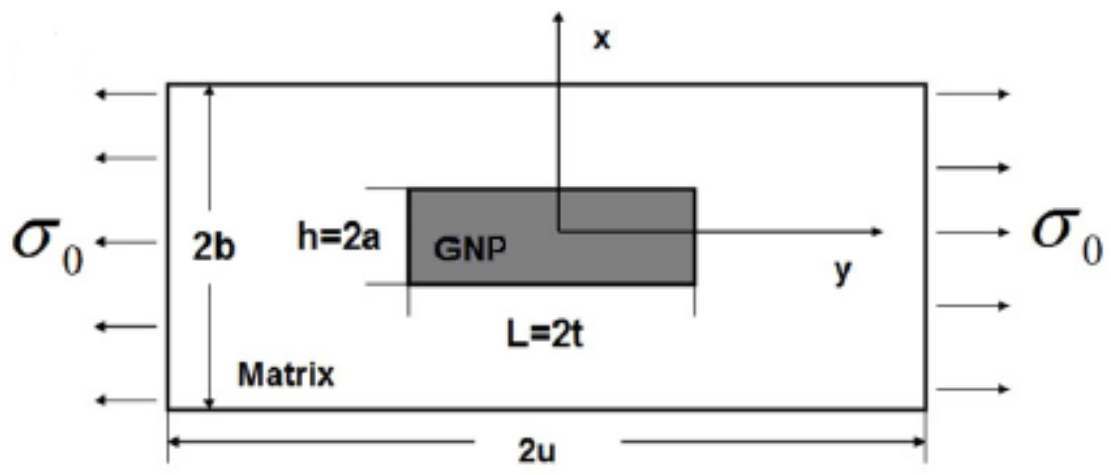

Figure 75: Schematic of GNP in a ceramic matrix used for the stress-transfer equations.

The values used in the computations include an average thickness of the GNP (h) of 7 $\mathrm{nm}$, a length (L) of $5 \mu \mathrm{m}$, an elastic modulus of the GNP $\left(\mathrm{E}_{\mathrm{G}}\right)$ of $750 \mathrm{GPa}$, a density of the GNP $\left(\rho_{\mathrm{G}}\right)$ of $1.82 \mathrm{~g} / \mathrm{cm}^{2}$ along with values of the other variables specific to each composition which are listed in Table 16 along with the resulting interfacial shear and axial stresses. Theoretical calculated values were used for the elastic modulus and the Poisson's ratio of the matrix.

Table 16: Values used in calculating the interfacial shear and axial stresses in the varying compositions along with the resulting stresses.

\begin{tabular}{|c|c|c|c|c|c|c|c|c|}
\hline Sample & $\begin{array}{c}\mathrm{E}_{\mathrm{m}} \\
(\mathrm{GPa})\end{array}$ & $v_{\mathrm{m}}$ & $\begin{array}{c}\rho_{\mathrm{m}} \\
\left(\mathrm{g} / \mathrm{cm}^{2}\right)\end{array}$ & $\Phi$ & $\mathrm{w}_{\mathrm{G}}$ & $\sigma_{0}$ & $\tau_{\max }$ & $\sigma_{\max }$ \\
\hline TaC-NbC- & 429.8 & 0.234 & 12.36 & 0.127 & 0.0045 & 129.8 & 132 & 186 \\
\hline 3GNP & 429.1 & 0.232 & 11.85 & 0.013 & 0.0047 & 150.0 & 158 & 213 \\
\hline TaC-NbC- & & & & & & & \\
\hline TaC-NbC- & 415.4 & 0.235 & 11.84 & 0.010 & 0.0047 & 212.5 & 226 & 307 \\
\hline 5Si-3GNP & & & & & & & & \\
\hline
\end{tabular}


Experimental values of the tensile fracture strength $\left(\sigma_{0}\right)$ from the diametral compression tests were used for each composition. The resulting maximum shear stress occurred at the edges of the GNP and the maximum axial stress occurred at the center of the GNP. Zhang et al. [19] identified the four possibilities for interfacial behavior which are listed in Table 17.

Table 17: Interfacial behavior possibilities between a GNP and the ceramic matrix.

\begin{tabular}{|c|c|l|}
\hline & Interfacial Behavior & \multicolumn{1}{c|}{ Result } \\
\hline $\mathbf{1}$ & $\tau_{\max }<\tau_{\text {interface }}$ and $\sigma_{\max }<\sigma_{\text {interface }}$ & $\begin{array}{l}\text { No interfacial delamination or GNP rupture } \\
\text { will occur }\end{array}$ \\
\hline $\mathbf{2}$ & $\tau_{\max }>\tau_{\text {interface }}$ and $\sigma_{\max }<\sigma_{\text {interface }}$ & $\begin{array}{l}\text { Interface will delaminate but no GNP rupture } \\
\text { will occur }\end{array}$ \\
\hline $\mathbf{3}$ & $\tau_{\max }<\tau_{\text {interface }}$ and $\sigma_{\max }>\sigma_{\text {interface }}$ & $\begin{array}{l}\text { No interfacial delamination, however GNP } \\
\text { rupture will occur }\end{array}$ \\
\hline $\mathbf{4}$ & $\tau_{\max }>\tau_{\text {interface }}$ and $\sigma_{\max }>\sigma_{\text {interface }}$ & $\begin{array}{l}\text { Both interfacial delamination and GNP rupture } \\
\text { will occur }\end{array}$ \\
\hline
\end{tabular}

Based on these possibilities and looking at both the maximum interfacial shear and stress results and the fracture surfaces resulting from diametral compression testing, the interfacial behavior can be characterized. Type 3 and 4 interfacial behaviors are not expected due to the very high tensile strength of GNP which should not lead to rupture. In the TaC-NbC-3GNP sample, interfacial delamination was visible and therefore exhibited type 2 interfacial behavior. The low interfacial shear strength likely resulted from the density only being $87 \%$ which resulted in poor surface area interaction between the GNP and TaC-NbC grains. In the TaC-NbC-5B ${ }_{4} \mathrm{C}-3 \mathrm{GNP}$ sample, there was greater surface interaction between the GNP and $\mathrm{TaC}-\mathrm{NbC}$ grains which resulted in greater 
maximum interfacial shear and axial strength. In the TaC-NbC-5Si-3GNP sample, the GNP surfaces appeared to interact with the formed SiC creating very strong interfacial bonding. In both of the samples with sintering additive addition, shearing between GNP layers was observed (GNP pullout) instead of shearing and delamination between the GNP and the surrounding ceramic matrix which points to type 1 interfacial behavior.

\subsection{Oxidation Analysis}

While the main objective of this research was to look at the mechanical responses of adding nanofillers to the $\mathrm{TaC}-\mathrm{NbC}$ system, a brief analysis was performed to check how the addition of the nanofillers affected the oxidation. For the oxidation study, testing was performed on only two samples: Tantalum-Niobium Carbide with the addition of 5 vol.\% Si as well as Tantalum-Niobium Carbide with the addition of both 5 vol.\% Si and 3 vol.\% GNP. These samples were chosen based on their greater toughness compared with the others.

The test was performed for 3 minutes at a standoff distance of $75 \mathrm{~mm}$ from the

plasma gun. The temperature profile (Figure 76) was measured using a thermocouple that was in contact with the back of the sample and the front side temperature was measured to be about $2500^{\circ} \mathrm{C}$ by AccuraSpray. Based on the temperature profiles, the sample with GNP addition resulted in a faster temperature increase as well as a higher temperature reading. This was expected due to the higher thermal conductivity of the added GNP. 


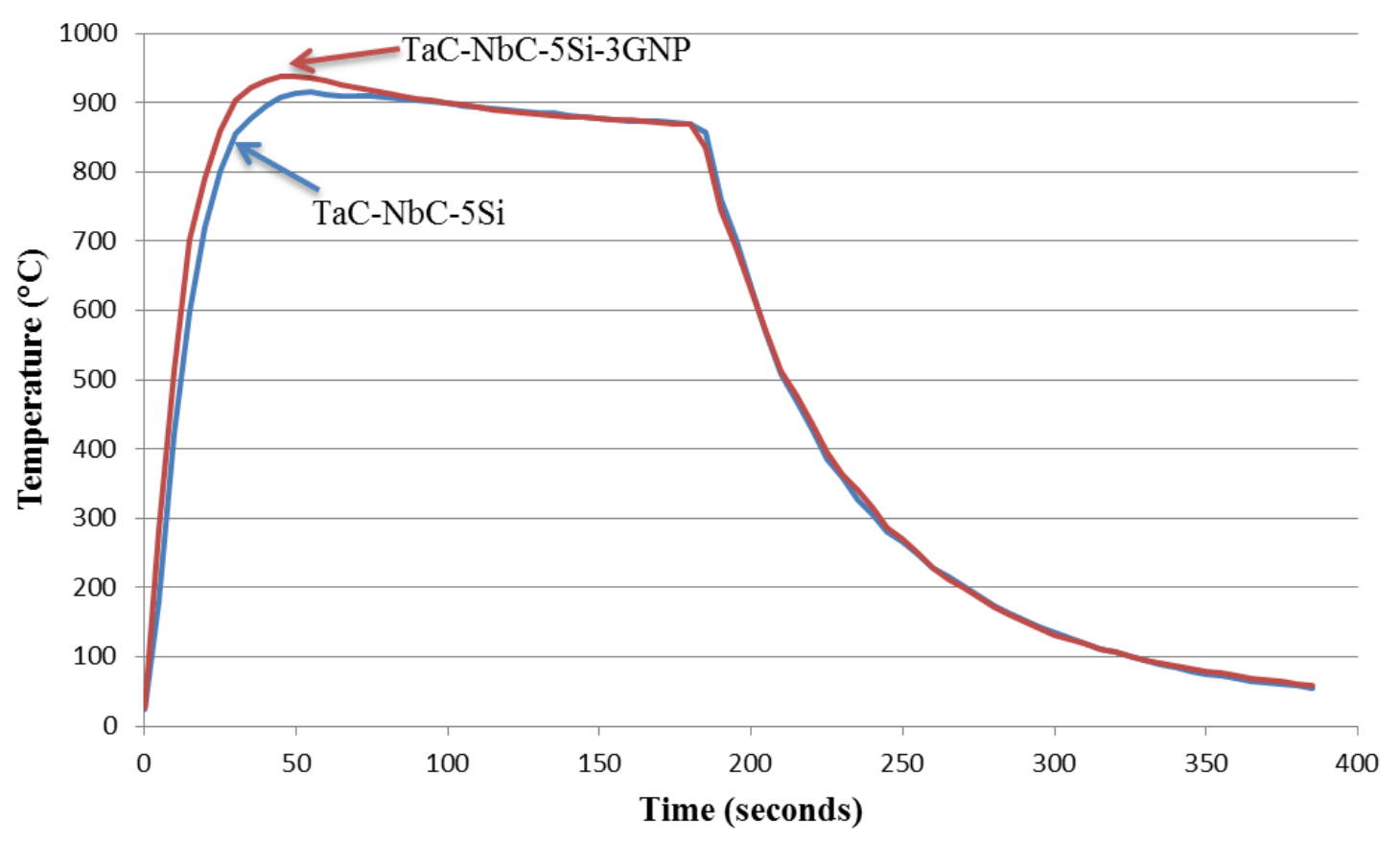

Figure 76: Temperature profile during oxidation testing measured by thermocouple in contact with back side of sample.

Both of the tested samples had a measured oxidation layer thickness of $0.29 \mathrm{~mm}$, however a visible difference was observed when qualitatively comparing the surfaces of the oxidation layers. Figure 77 shows the front side surface of the oxidation layer for the TaC-NbC-5Si sample. As can be seen from the SEM imaging, the oxidation layer displayed many cracks and ruptures. In comparison, the front side surface of the oxidation layer for the sample with GNP addition (Figure 78), displayed a more intact top layer with cracks that were less pronounced. Therefore, a conclusion can be made that with the addition of GNP, the oxidized surface would provide greater protection to the material underneath resulting in less susceptibility to oxidation failure. 


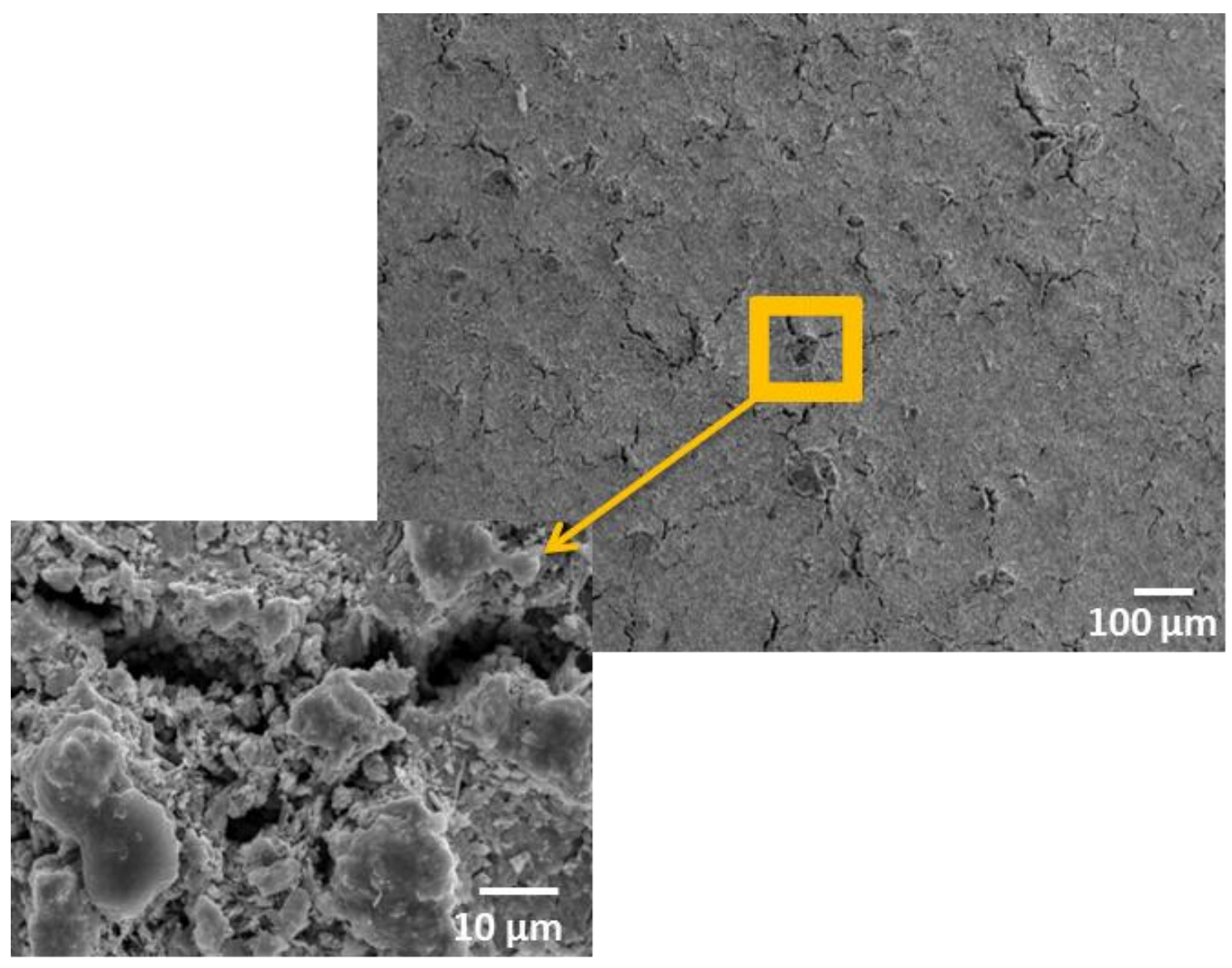

Figure 77: SEM micrograph of the front side oxidation layer surface of the TaC-NbC-5 vol.\% Si sample. 


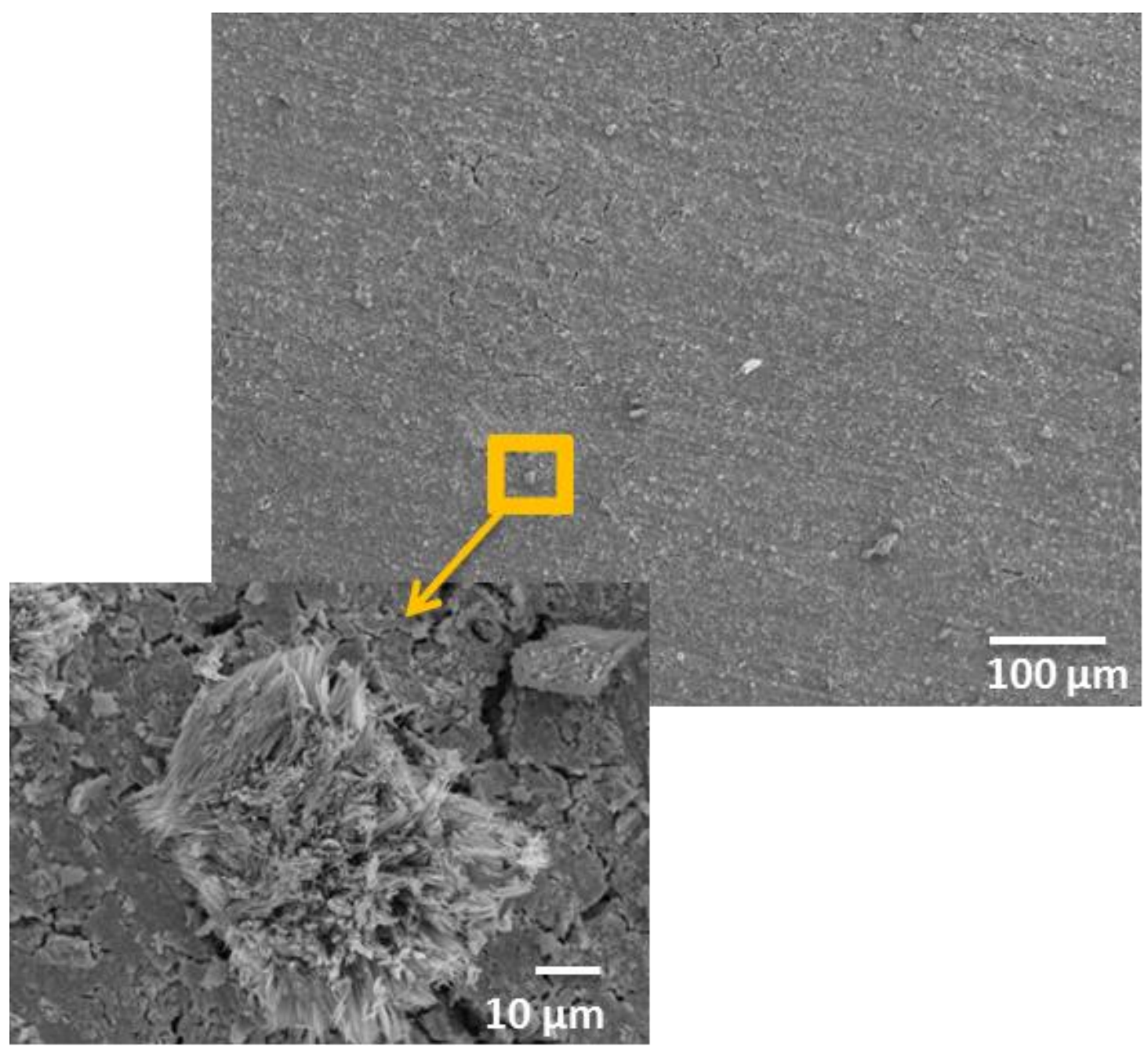

Figure 78: SEM micrograph of the front side oxidation layer surface of the TaC-NbC-5 vol.\% Si-3 vol.\% GNP sample. 


\section{Chapter V: Conclusions}

This dissertation presents an enhanced potential for GNP reinforced tantalumniobium carbide ceramic composites to be used in applications that experience extreme environmental conditions. The major setbacks to the implementation of UHTC ceramics, being low fracture toughness and reliability issues due to processing defects, have been examined. Investigation into the fundamental GNP deformation mechanisms by indentation on bulk spark plasma sintered GNP revealed their anisotropic behavior. Analysis of the deformation mechanisms, aided by performing these tests in-situ, led to an understanding of which mechanisms are prevalent in each orientation. In addition, a quantitative comparison was made between the associated energy dissipation capabilities

of each mechanism. Investigation into the role of powder processing as well as SPS parameter variation resulted in improvement in both microstructure and mechanical properties of the sintered $\mathrm{TaC}-\mathrm{NbC}$. Sintering additives resulted in the achievement of near full relative densification and the secondary phase formations were analyzed. GNP reinforced ceramic composites with near full relative density were sintered and experimentation revealed the mechanisms of toughening. The specific conclusions on GNP reinforcement to UHTC materials and the potential for the TaC-NbC ceramic composites to be use in extreme environment conditions are listed below:

- Consolidation of both bulk GNP and GNP reinforced TaC-NbC composites resulted in an aligned orientation of the GNP perpendicular to the SPS pressing axis. This enabled the study of the anisotropy of the toughening mechanisms inherent to the GNP. 
- When indenting bulk GNP under displacement control, the energy required to reach the same indentation depth was $270 \%$ greater when indenting in the orthogonal GNP orientation compared to the energy required when indenting in the surface orientation. This leads to the conclusion that when GNP is used as reinforcement in a ceramic composite, the preferred orientation for increased toughness would be orthogonal to the surface.

- The energy dissipation mechanisms in the surface orientation of bulk GNP (in order of frequency of occurrence) are sheet sliding followed by bending, kinking, and sheet pull-out. The energy dissipation mechanisms in the orthogonal orientation of bulk GNP (in order of frequency of occurrence) are bending, pushout, and pop-out. The sheet sliding mechanism was found to dissipate the least energy due to the weak van der Waals forces and therefore explains why the total dissipated energy in the surface orientation was much lower than in the orthogonal orientation.

- The use of sintering additive $\left(5\right.$ vol. $\% \mathrm{~B}_{4} \mathrm{C}$ or $\left.\mathrm{Si}\right)$ resulted in near full relative densification $(>99 \%)$ of the $\mathrm{TaC}-\mathrm{NbC}$ ceramic composites. Because any areas of non-uniformity are areas where failure is likely to occur, the reliability of the composites increased as a result of reducing the porosity as well as by refining the microstructure by analyzing the instantaneous densification during the spark plasma sintering process.

- Relative fracture toughness evaluation performed by calculating the residual damaged area after indentation on samples with sintering aid addition showed a 
$46 \%$ increase in toughness when $\mathrm{Si}$ is used as the sintering additive compared with $\mathrm{B}_{4} \mathrm{C}$.

- $\quad$ SPS processed TaC-NbC-5Si-3GNP composites, with both 5 vol.\% Si added as sintering additive and 3 vol.\% GNP added as a reinforcement phase, had a reaction occur between the added $\mathrm{Si}$ and most of the GNP to form $\mathrm{SiC}$. The $\mathrm{SiC}$ appeared to have formed at the areas where the GNP were already aligned resulting in the alignment of the secondary phase of $\mathrm{SiC}$.

- The relative fracture toughness in the GNP reinforced ceramic composites was nearly doubled in the orthogonal direction when compared to the surface orientation.

- The ability to run the high load indentation experiments in situ resulted in enhanced analysis of the intrinsic toughening mechanisms of GNP reinforcement by shedding light on the frequency of the mechanisms occurrence. When indentation was performed on GNP reinforced TaC-NbC composites with GNP aligned in the surface orientation, the predominant toughening mechanisms were sheet sliding, crack bridging, and crack arrest. When indentation was performed with GNP aligned in the orthogonally aligned orientation, the predominant toughening mechanisms were crack bridging and crack deflection.

- While the crack bridging GNP toughening mechanism was observed when indenting on both alignment orientations, it was clear that a greater energy was dissipated when a crack is bridged in the orthogonal alignment due to the crack being forced to propagate below the GNP in three dimensions. 
- Diametral compression testing of GNP reinforced composites revealed an increase in relative fracture strength of $16 \%$ when $\mathrm{B}_{4} \mathrm{C}$ was used as the sintering additive and an increase of $64 \%$ when $\mathrm{Si}$ was used as the sintering additive when compared to no sintering additive being used. 


\section{Chapter VI: Recommendations for Future Work}

This research focused on enhancing the potential for UHTC materials to be used in applications that experience extreme environmental conditions. By experimenting with the sintering parameters, the reliability of the ceramic system was improved by refining the microstructure and uniformity. With the use of GNP as a reinforcement material, the toughness of the normally very brittle ceramic was enhanced. While the findings of this research provide enhanced potential for reinforced UHTC materials for the desired applications, some topics require further investigation to progress towards the eventual implementation of nanofiller reinforced UHTC materials. The following is a list of recommendations for future work.

\subsection{Analysis of SiC Reaction between $\mathrm{Si}$ and GNP}

Further investigation into the reaction occurring between the GNP added for reinforcement and the Si added as a sintering additive has the potential to further increase the toughness of the composites. This research showed that the secondary phase of SiC had an aligned orientation due to the Si reacting with the already aligned GNP and that the toughness increase was anisotropic. With a complete understanding of when the transition from $\beta \rightarrow \alpha \mathrm{SiC}$ occurs and how the laminar structure of the GNP affects the formation of elongated $\mathrm{SiC}$ grains, there is the potential to obtain an even greater overall toughness. 
6.2 Transmission Electron Microscopy (TEM) Analysis for a Greater Understanding of the Stress Transfer between the Ceramic Matrix and the GNP Reinforcement.

This study presented a preliminary investigation into the interfacial stress transfer between the ceramic matrix and the GNP. TEM analysis should increase the understanding of the stress transfer that occurs by investigating the bonding between the phases.

\subsection{Crack Propagation Viewing}

Ceramics are notoriously difficult to obtain accurate fracture toughness values due to their brittle nature leading to extremely rapid crack propagation. There are two methods warranting exploration for crack propagation viewing. The first is using very slow loading rates and doing in situ indentation with a Vickers tip. In this research, in situ indentation was performed using a cono-spherical tip which made it impossible to predict where crack propagation would occur. With a Vickers tip, the crack will propagate from one of the four sharp edges which gives a high probability of capturing the crack growth. The other method for crack propagation viewing is using an in situ double torsion testing technique. In one of my previous publications [78], the a double torsion technique for brittle ceramics was proposed using the in situ load frame. Initial investigation was carried out using 3D printed fixtures; however more testing is still needed to come up with a completed experimental plan. Both of these proposed methods provide a framework to characterize crack growth rate, fracture toughness, and stress intensity factors that have the potential to improve the analysis of a material's service lifetime. 


\subsection{Investigation into Alternative Ceramic Stoichiometries and Compositions}

In this research, only the pre-alloyed ceramic system made up of 80 weight percent $\mathrm{TaC}$ and 20 weight percent $\mathrm{NbC}$ was investigated. In future research it is recommended to try a higher weight percentage of $\mathrm{NbC}$ addition due to the benefit of its lower density. While this TaC-NbC composition has high chemical resistivity that makes it an ideal candidate for rocket nozzle throat inserts, various other compositions could lead to a greater oxidation resistance and toughness. In further research, it is recommended to try a combination including $\mathrm{HfC}$ which has a greater oxidation resistance compared to $\mathrm{TaC}$ in an oxygen rich environment.

\subsection{Further Exploration into Enhancing Oxidation Resistance}

Besides trying a new combination of ceramics, as stated previously, to increase the oxidation resistance, the oxidation resistance can also be increased by exploring the addition of other combinations of transition metals. Bongiorno et al. [79] found that the high cation field strengths of transition metal oxides can lead to good oxidation behavior by producing immiscible glasses upon oxidation. It could be worthwhile to investigate the addition of both $\mathrm{B}_{4} \mathrm{C}$ and $\mathrm{Si}$ as sintering additive to a single composition. 


\section{REFERENCES}

1. James, R., et al., A reusable space vehicle design study exploring sharp leading edges, in 35th AIAA Thermophysics Conference. 2001, American Institute of Aeronautics and Astronautics.

2. Squire, T.H. and J. Marschall, Material property requirements for analysis and design of UHTC components in hypersonic applications. Journal of the European Ceramic Society, 2010. 30(11): p. 2239-2251.

3. Dean, K., G. Ken, and P. Dinesh, Temperature constraints at the sharp leading edge of a Crew Transfer Vehicle, in 35th AIAA Thermophysics Conference. 2001, American Institute of Aeronautics and Astronautics.

4. [cited 2016 May 02]; Space Shuttle Re-Entry Figure]. Available from: http://picsabout-space.com/space-shuttle-re-entry-temperature-of? $\mathrm{p}=4$.

5. [cited 2016 May 02]; Rocket Nozzle Figure]. Available from: http://www.mineralstech.com/Pages/Minteq/Other-MTO.aspx.

6. Upadhya, K., Yang, J.M., Hoffman, W., Advanced Materials for Ultrahigh Temperature Structural Applications Above 2000C. 1997.

7. Pierson, H.O., 5 - Carbides of Group V: Vanadium, Niobium and Tantalum Carbides, in Handbook of Refractory Carbides and Nitrides. 1996, William Andrew Publishing: Westwood, NJ. p. 81-99.

8. E. Wuchina, E.O., M. Opeka, W. Fahrenholtz, I. Talmy, UHTCs: Ultra-High Temperature Ceramic Materials for Extreme Environment Applications. Electrochem. Soc. Interface, 2007. Winter: p. 30-36.

9. Allen, M.J., V.C. Tung, and R.B. Kaner, Honeycomb Carbon: A Review of Graphene. Chemical Reviews, 2010. 110(1): p. 132-145.

10. Frank, I.W., et al., Mechanical properties of suspended graphene sheets. Journal of Vacuum Science \& Technology B: Microelectronics and Nanometer Structures, 2007. 25(6): p. 2558.

11. Lee, C., et al., Measurement of the elastic properties and intrinsic strength of monolayer graphene. Science, 2008. 321(5887): p. 385-8.

12. M.A. Rafiee, J.R., Z. Wang, H. Song, Z.Z. Yu, N. Koratkar, Enhanced mechanical properties of nanocomposites at low graphene content. ACS Nano, 2009. 3(12): p. 3884-3890. 
13. Seiner, H., et al., Anisotropic elastic moduli and internal friction of graphene nanoplatelets/silicon nitride composites. Composites Science and Technology, 2013. 75: p. 93-97.

14. Yang, S.B., et al., Fabrication of Cobalt and Cobalt Oxide/Graphene Composites: Towards High-Performance Anode Materials for Lithium Ion Batteries. Chemsuschem, 2010. 3(2): p. 236-239.

15. Choi, W., et al., Synthesis of Graphene and Its Applications: A Review. Critical Reviews in Solid State and Materials Sciences, 2010. 35(1): p. 52-71.

16. Kotov, N.A., Materials science: Carbon sheet solutions. Nature, 2006. 442(7100): p. 254-255.

17. Liu, J., H. Yan, and K. Jiang, Mechanical properties of graphene plateletreinforced alumina ceramic composites. Ceramics International, 2013. 39(6): p. 6215-6221.

18. Nieto, A., D. Lahiri, and A. Agarwal, Nanodynamic mechanical behavior of graphene nanoplatelet-reinforced tantalum carbide. Scripta Materialia, 2013. 69(9): p. 678-681.

19. Zhang, L., et al., Interfacial stress transfer in a graphene nanosheet toughened hydroxyapatite composite. Applied Physics Letters, 2014. 105(16): p. 161908.

20. Chen, F., et al., Field assisted sintering of graphene reinforced zirconia ceramics. Ceramics International, 2015. 41(4): p. 6113-6116.

21. Nieto, A., D. Lahiri, and A. Agarwal, Graphene NanoPlatelets reinforced tantalum carbide consolidated by spark plasma sintering. Materials Science and Engineering: A, 2013. 582: p. 338-346.

22. J.D. Bull, D.J.R., C.C. Karika. Stability characterization of diboride composites under high velocity atmospheric flight conditions. in 24th International SAMPE Technical Conference. 1992.

23. S.R. Levine, M.S., E.J. Opila, J.A. Lorincz, J. Petko, D.T. Ellerby, M.J. Gasch. Ultra-High Temperature Ceramic Composites for Leading Edges. in JANNAF 39th Combustion/27th Airbreathing Propulsion/21st Propulsion Systems Hazards/3rd Modeling and Simulation Joint Subcommittee Meeting. 2003. Colorado Springs.

24. Booker, P., and Rudy, E., Phase Equilibria Investigations of Binary, Ternary, and Higher Order Systems. Part III. Phase Equilibria Studies in the Nb-Ta-C System. 1970 . 
25. Metcalfe, A.E., Norbert, Rocket Nozzle Material, USPTO, Editor. 2006, HIZ Corporation.

26. Hackett, K., et al., Phase Constitution and Mechanical Properties of Carbides in the Ta-C System. Journal of the American Ceramic Society, 2009. 92(10): p. 2404-2407.

27. M. Desmaison-Brut, N.A., J. Desmaison, Comparison of the Oxidation Behavior of Two Dense Hot Isostatically Pressed Tantalum Carbide (TaC and Ta2C Materials. Journal of the European Ceramic Society, 1997. 17: p. 1325-1334.

28. Zhang, X., G.E. Hilmas, and W.G. Fahrenholtz, Densification, Mechanical Properties, and Oxidation Resistance of TaC-TaB2Ceramics. Journal of the American Ceramic Society, 2008. 91(12): p. 4129-4132.

29. Zhang, X., G.E. Hilmas, and W.G. Fahrenholtz, Densification and mechanical properties of TaC-based ceramics. Materials Science and Engineering: A, 2009. 501(1-2): p. 37-43.

30. Zhang, X., et al., Hot Pressing of Tantalum Carbide With and Without Sintering Additives. Journal of the American Ceramic Society, 2007. 90(2): p. 393-401.

31. Sciti, D., et al., Processing, mechanical properties and oxidation behavior of TaC and HfC composites containing 15 vol\% TaSi2 or MoSi2. Journal of Materials Research, 2011. 24(06): p. 2056-2065.

32. Liu, J.-X., Y.-M. Kan, and G.-J. Zhang, Pressureless Sintering of Tantalum Carbide Ceramics without Additives. Journal of the American Ceramic Society, 2010. 93(2): p. 370-373.

33. Talmy, I.G., J.A. Zaykoski, and M.M. Opeka, Synthesis, processing and properties of TaC-TaB2-C ceramics. Journal of the European Ceramic Society, 2010. 30(11): p. 2253-2263.

34. Balani, K., et al., Synthesis, Microstructural Characterization, and Mechanical Property Evaluation of Vacuum Plasma Sprayed Tantalum Carbide. Journal of the American Ceramic Society, 2006. 89(4): p. 1419-1425.

35. Kim, B.-R., et al., Mechanical properties and rapid consolidation of binderless nanostructured tantalum carbide. Ceramics International, 2009. 35(8): p. 33953400 .

36. Kim, B.-R., et al., Mechanical properties and rapid consolidation of binderless niobium carbide. Journal of Alloys and Compounds, 2009. 481(1-2): p. 573-576. 
37. Bakshi, S.R., et al., Spark plasma sintered tantalum carbide: Effect of pressure and nano-boron carbide addition on microstructure and mechanical properties. Materials Science and Engineering: A, 2011. 528(3): p. 1287-1295.

38. Khaleghi, E., et al., Spark plasma sintering of tantalum carbide. Scripta Materialia, 2010. 63(6): p. 577-580.

39. Liu, H., et al., Microstructure and mechanical properties of the spark plasma sintered TaC/SiC composites: Effects of sintering temperatures. Journal of the European Ceramic Society, 2012. 32(13): p. 3617-3625.

40. Liu, L., F. Ye, and Y. Zhou, New route to densify tantalum carbide at $1400^{\circ} \mathrm{C}$ by spark plasma sintering. Materials Science and Engineering: A, 2011. 528(13-14): p. $4710-4714$.

41. Suarez, M., et al., Challenges and Opportunities for Spark Plasma Sintering: A Key Technology for a New Generation of Materials. 2013.

42. Rahaman, M.N., Ceramic Processing and Sintering. 2 ed. 2003, New York: Marcel Dekker, Inc.

43. Guillon, O., et al., Field-Assisted Sintering Technology/Spark Plasma Sintering: Mechanisms, Materials, and Technology Developments. Advanced Engineering Materials, 2014. 16(7): p. 830-849.

44. Liu, L., et al., Densification of tantalum carbide ceramics with $5 \mathrm{~mol} . \% \mathrm{Al}, \mathrm{Cu}, \mathrm{Ag}$ and $A u$. Scripta Materialia, 2013. 69(8): p. 574-577.

45. Gibson, J.O., Gibson, M.G., Production of Carbon Fiber-Tantalum Carbide Composites, U.S. Patent, Editor. 1979.

46. Liu, L., et al., Microstructure and mechanical properties of the spark plasma sintered TaC/SiC composites. Materials Science and Engineering: A, 2011. 529: p. 479-484.

47. Guicciardi, S., et al., Microstructure and Toughening Mechanisms in Spark Plasma-Sintered ZrB2 Ceramics Reinforced by SiC Whiskers or SiC-Chopped Fibers. Journal of the American Ceramic Society, 2010. 93(8): p. 2384-2391.

48. Kaushik, B.K. and M.K. Majumder, Carbon Nanotube: Properties and Applications. 2015: p. 17-37.

49. Bakshi, S.R., et al., Spark plasma sintered tantalum carbide-carbon nanotube composite: Effect of pressure, carbon nanotube length and dispersion technique on microstructure and mechanical properties. Materials Science and Engineering: A, 2011. 528(6): p. 2538-2547. 
50. Sivakumar, R., et al., Thermal conductivity in multi-wall carbon nanotube/silicabased nanocomposites. Scripta Materialia, 2007. 56(4): p. 265-268.

51. Lahiri, D., et al., Graphene-induced strengthening in spark plasma sintered tantalum carbide-nanotube composite. Scripta Materialia, 2013. 68(5): p. 285288.

52. Rafiee, M.A., Rafiee, J., Wang, Z., Song, H., Yu, Z.Z., Koratkar, N., Enhanced mechanical properties of nanocomposites at low graphene content. ACS Nano, 2009. 3(12): p. 3884-3890.

53. Eizenberg, M. and J.M. Blakely, Carbon monolayer phase condensation on Ni(111). Surface Science, 1979. 82(1): p. 228-236.

54. Aizawa, T., et al., Anomalous Bond of Monolayer Graphite on Transition-Metal Carbide Surfaces. Physical Review Letters, 1990. 64(7): p. 768-771.

55. Berger, C., et al., Electronic confinement and coherence in patterned epitaxial graphene. Science, 2006. 312(5777): p. 1191-1196.

56. Novoselov, K.S., et al., Electric field effect in atomically thin carbon films. Science, 2004. 306(5696): p. 666-669.

57. Stankovich, S., et al., Synthesis and exfoliation of isocyanate-treated graphene oxide nanoplatelets. Carbon, 2006. 44(15): p. 3342-3347.

58. Balandin, A.A., et al., Superior thermal conductivity of single-layer graphene. Nano Letters, 2008. 8(3): p. 902-907.

59. Stankovich, S., et al., Graphene-based composite materials. Nature, 2006. 442(7100): p. 282-6.

60. Walker, L.S., et al., Toughening in Graphene Ceramic Composites. Acs Nano, 2011. 5(4): p. 3182-3190.

61. Kvetkova, L., et al., Fracture toughness and toughening mechanisms in graphene platelet reinforced Si3N4 composites. Scripta Materialia, 2012. 66(10): p. 793796.

62. Chou, Y.S. and D.J. Green, Silicon-Carbide Platelet Alumina Composites .1. Effect of Forming Technique on Platelet Orientation. Journal of the American Ceramic Society, 1992. 75(12): p. 3346-3352.

63. Porwal, H., et al., Graphene reinforced alumina nano-composites. Carbon, 2013. 64: p. 359-369. 
64. Ramirez, C., et al., Electrical conductivity maps in graphene nanoplatelet/silicon nitride composites using conducting scanning force microscopy. Carbon, 2011. 49(12): p. 3873-3880.

65. Dai, G. and L. Mishnaevsky, Graphene reinforced nanocomposites: 3D simulation of damage and fracture. Computational Materials Science, 2014. 95: p. 684-692.

66. xGnP-M_Data-Sheet. 2013, XG Sciences Inc: www.xgsciences.com.

67. Procopio, A.T., A. Zavaliangos, and J.C. Cunningham, Analysis of the diametrical compression test and the applicability to plastically deforming materials. Journal of Materials Science, 2003. 38(17): p. 3629-3639.

68. Fahrenholtz, W.G., Wuchina, E.J., Lee, W.E., Zhou, Y., Ultra-High Temperature Ceramics: Materials for Extreme Environment Applications. 2014, Hoboken, New Jersey: John Wiley \& Sons, Inc.

69. Zhong, L., et al., Transient liquid phase sintering of tantalum carbide ceramics by using silicon as the sintering aid and its effects on microstructure and mechanical properties. Materials Chemistry and Physics, 2015. 149-150: p. 505-511.

70. Silvestroni, L., et al., Strength and toughness: The challenging case of TaC-based composites. Composites Part B: Engineering, 2015. 72: p. 10-20.

71. Rudolf, C., B. Boesl, and A. Agarwal, In situ indentation behavior of bulk multilayer graphene flakes with respect to orientation. Carbon, 2015. 94: p. 872-878.

72. Lee, S.K., Y.C. Kim, and C.H. Kim, Microstructural Development and Mechanical-Properties of Pressureless-Sintered Sic with Plate-Like Grains Using Al2o3-Y2o3 Additives. Journal of Materials Science, 1994. 29(20): p. 5321-5326.

73. Lee, J.K., et al., Effect of starting phase on microstructure and fracture toughness of hot-pressed silicon carbide. Materials Letters, 2002. 57(1): p. 203-208.

74. Padture, N.P., In Situ-Toughened Silicon-Carbide. Journal of the American Ceramic Society, 1994. 77(2): p. 519-523.

75. Sciti, D., S. Guicciardi, and A. Bellosi, Effect of annealing treatments on microstructure and mechanical properties of liquid-phase-sintered silicon carbide. Journal of the European Ceramic Society, 2001. 21(5): p. 621-632.

76. Ma, J.P., et al., Raman analysis of a crystalline SiC sample prepared from carbon-saturated melt of silicon. Chinese Physics Letters, 2001. 18(8): p. 11231125. 
77. Padture, N.P. and B.R. Lawn, Toughness Properties of a Silicon-Carbide with an in-Situ Induced Heterogeneous Grain-Structure. Journal of the American Ceramic Society, 1994. 77(10): p. 2518-2522.

78. Rudolf, C., B. Boesl, and A. Agarwal, In Situ Mechanical Testing Techniques for Real-Time Materials Deformation Characterization. Jom, 2016. 68(1): p. 136142.

79. Bongiorno, A., et al., A perspective on modeling materials in extreme environments: Oxidation of ultrahigh-temperature ceramics. Mrs Bulletin, 2006. 31(5): p. 410-418. 


\section{APPENDICES}

Appendix 1: FIB cross-sections for grain size viewing

The following are the images used for average grain size calculations for the samples with GNP addition. A total of 50 grains were measured and the average was reported in Table 12 in section 4.4.2.

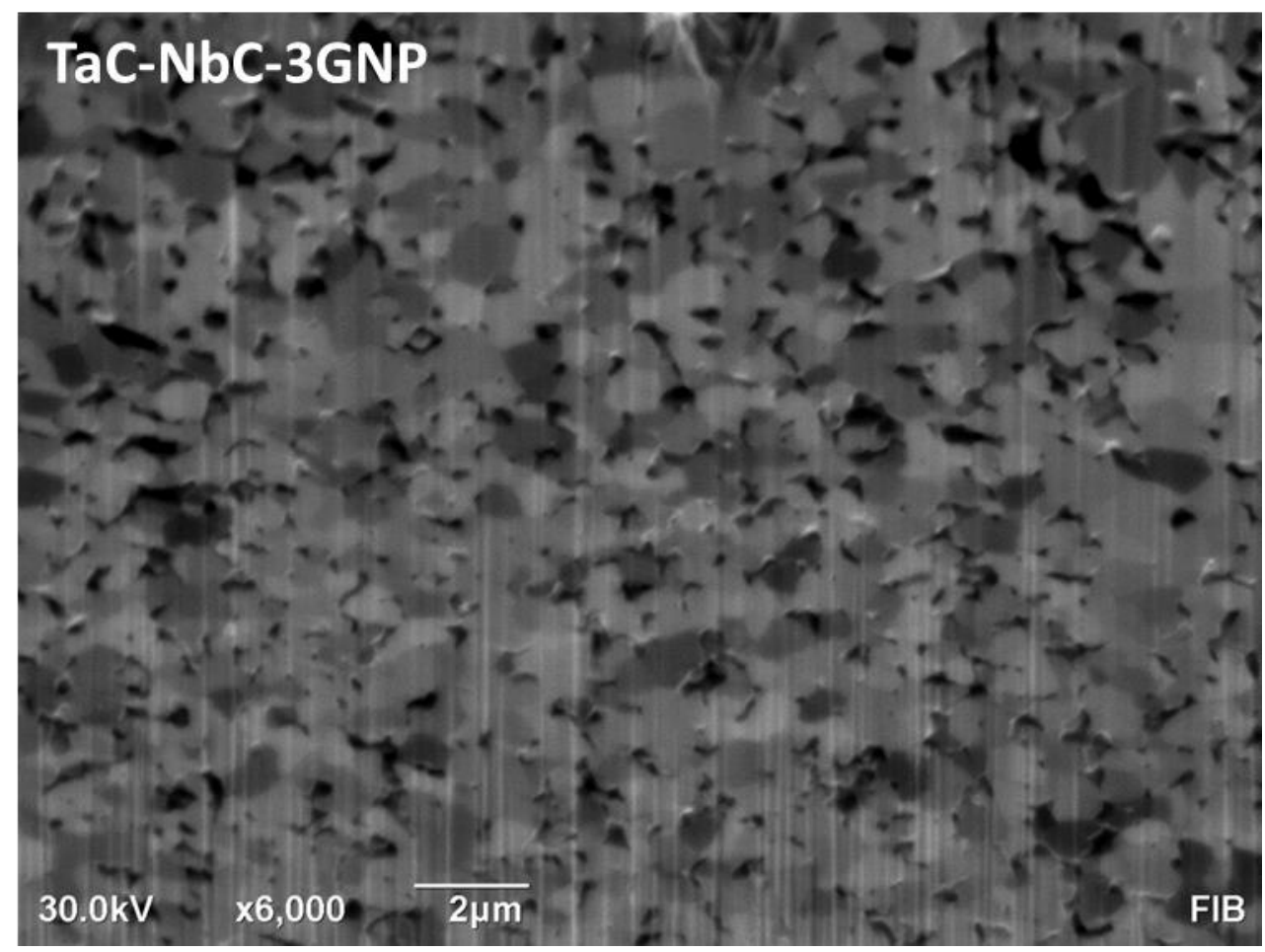

Figure A1: FIB cross section of TaC-NbC-3GNP for grain size viewing. 


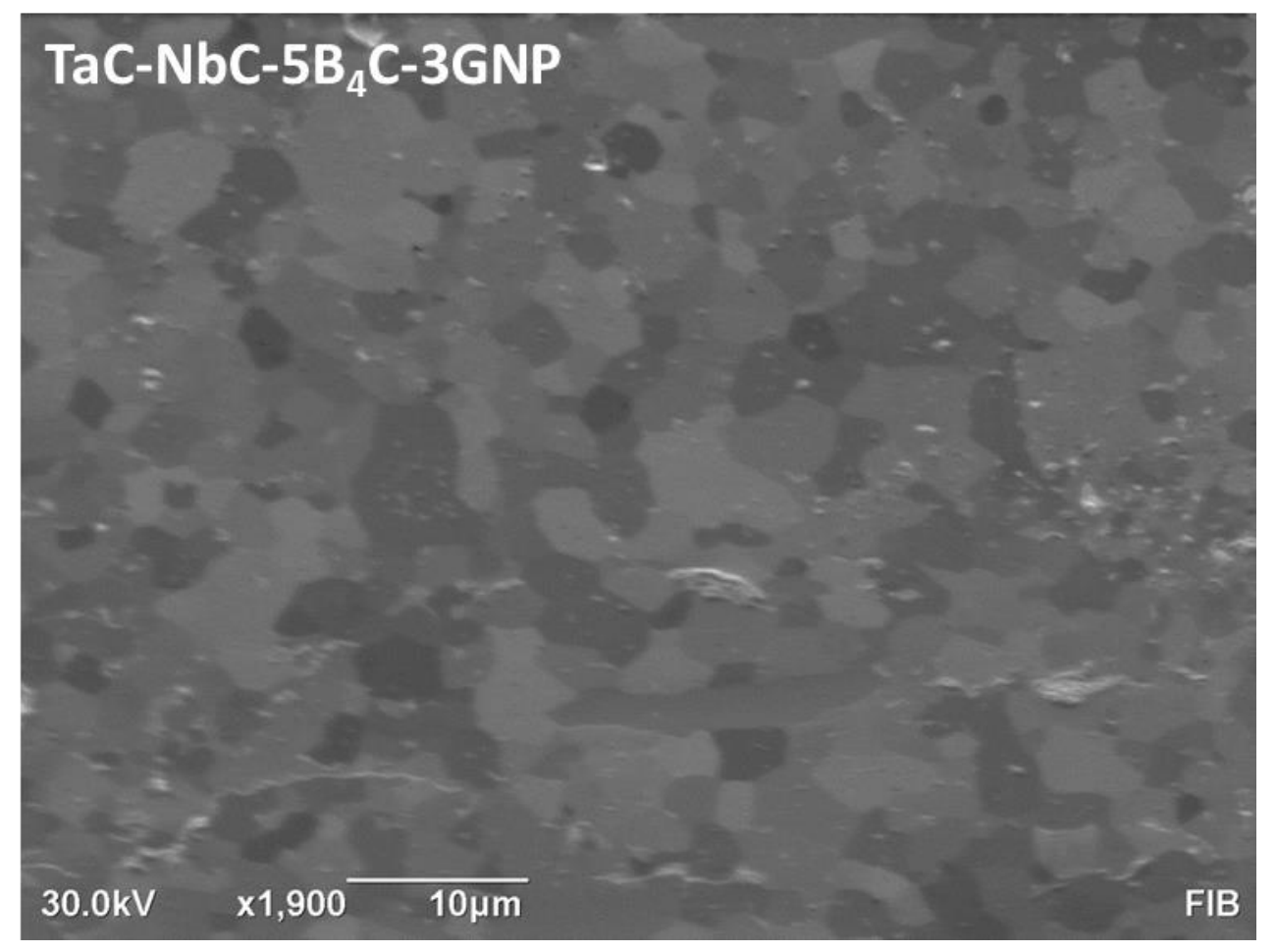

Figure A2: FIB cross section of TaC-NbC-5B 4 C-3GNP for grain size viewing.

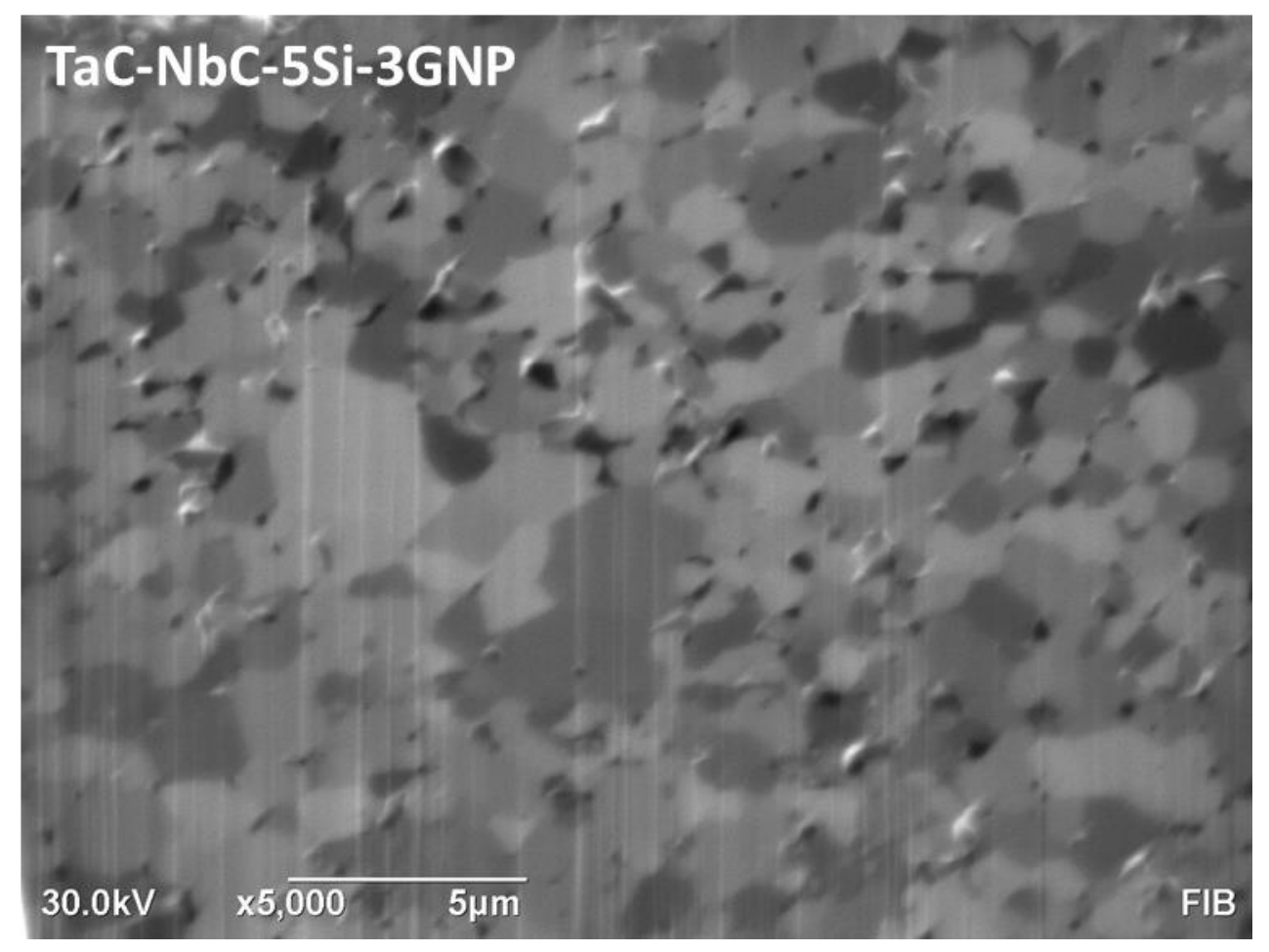

Figure A3: FIB cross section of TaC-NbC-5Si-3GNP for grain size viewing. 
Appendix 2: EDS analysis from samples with Si addition

The following is the EDS verification of the $\mathrm{SiC}$ phase forming from a reaction between the added silicon and the GNP:

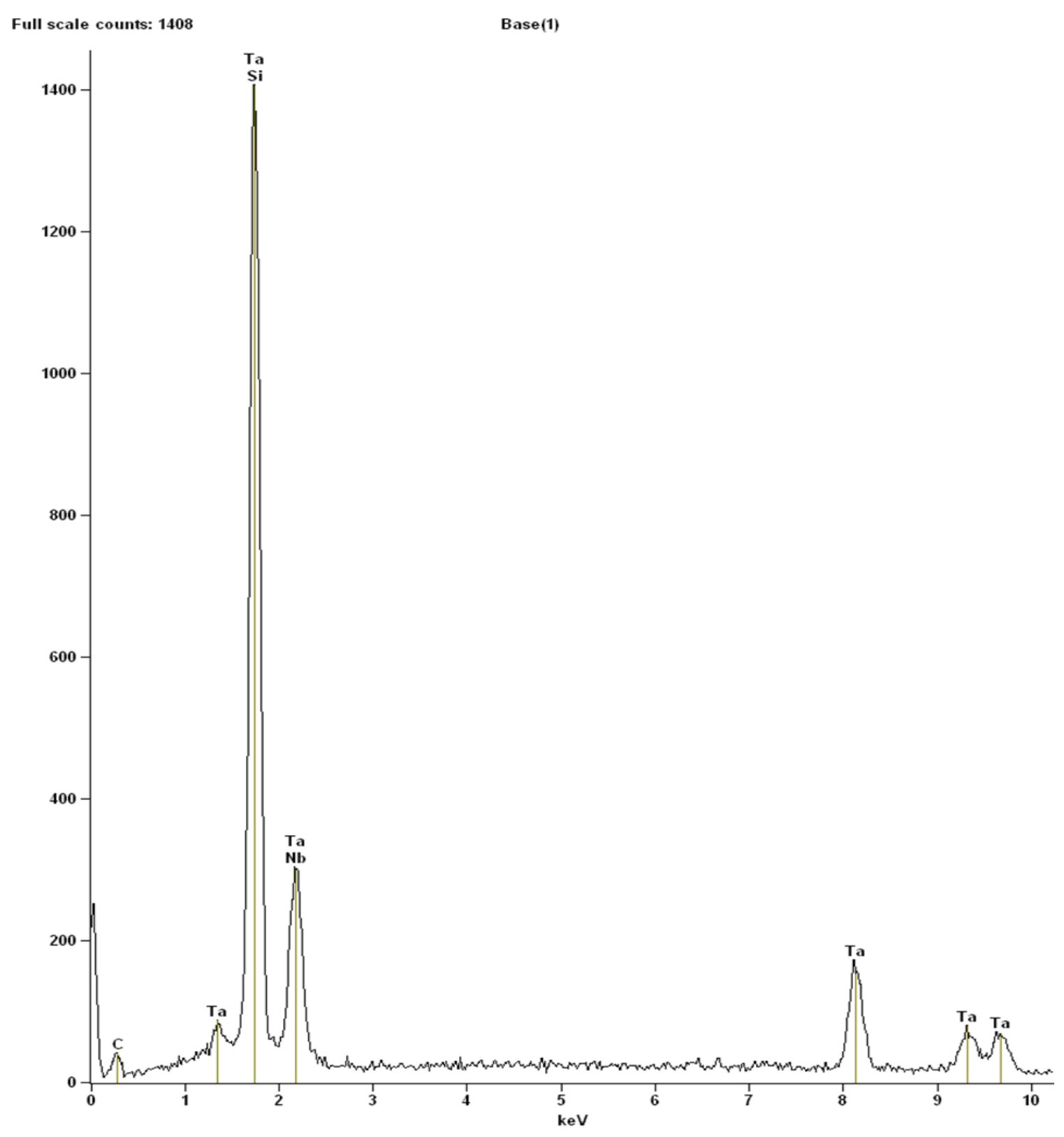



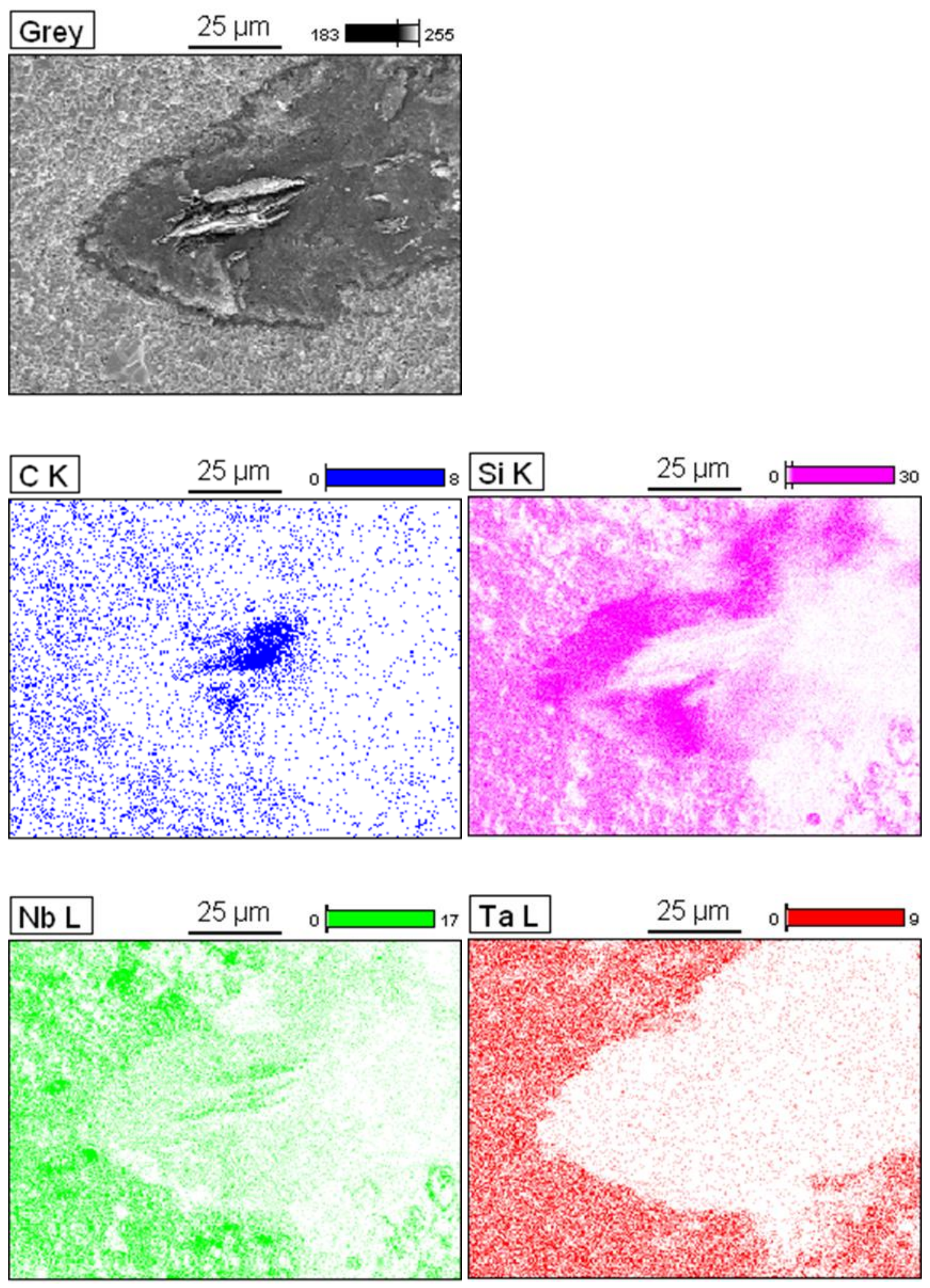
Appendix 3: This section lists the publications during the $\mathrm{PhD}$ research followed by the first pages of all first authored publications.

1. P. Nautiyal, Chris Rudolf, A. Loganathan, C. Zhang, B. Boesl, A. Agarwal. Directionally Aligned Ultra-long Boron Nitride Nanotube Induced Strengthening of Aluminum based Sandwich Composite. Adv. Eng. Mater. (Online: 20 July 2016)

2. Christopher C. Rudolf, B. Boesl, A. Agarwal. TaC-NbC Formed by Spark Plasma Sintering with the Addition of Sintering Additives. J. Ceram. Soc. Japan 124 (2016) 381-387.

3. M. Asadikiya, Christopher Rudolf, C. Zhang, B. Boesl, Y. Zhong. The Role of CALPHAD Approach in the Sintering of B4C with SiC as a Sintering Aid by Spark Plasma Sintering Technique. 11th International Conference on Ceramic Materials \& Components for Energy \& Environmental Applications, February 2016.

4. Chris Rudolf, B. Boesl, A. Agarwal. In Situ Mechanical Testing Techniques for RealTime Materials Deformation Characterization. JOM 68(1) (2016) 136-142.

5. Chris Rudolf, B. Boesl, A. Agarwal. In situ indentation behavior of bulk multi-layer graphene flakes with respect to orientation. Carbon 94 (2015) 872-878. 


\title{
$\mathrm{TaC}-\mathrm{NbC}$ formed by spark plasma sintering with the addition of sintering additives
}

\author{
Christopher C. RUDOLF, Arvind AGARWAL ${ }^{\dagger}$ and Benjamin BOESL ${ }^{\dagger \dagger}$ \\ Plasma Forming Laboratory Department of Mechanical and Materials Engineering Florida International University, \\ Miami, FL, 33174, USA
}

\begin{abstract}
$\mathrm{TaC}-\mathrm{NbC}$ with and without the addition of 5 vol.\% $\mathrm{B}_{4} \mathrm{C}$ and $5 \mathrm{vol} . \%$ Si nano-powders as sintering aids were consolidated by spark plasma sintering at $1850^{\circ} \mathrm{C}$. The effect of sintering aid addition on the densification and mechanical properties is evaluated along with secondary phase formations. Relative density $>99 \%$ is achieved with a hold time of just 3 min for the addition of Si and $10 \mathrm{~min}$ for the addition of $\mathrm{B}_{4} \mathrm{C}$ as sintering additives. High load instrumented indentation was performed and projected area of residual da mage is compared to estimate rehative fracture toughness. The addition of $5 \mathrm{vol} . \% \mathrm{Si}$ as a sintering additive resulted in a $46 \%$ reduction in projected residual damage area and $14.5 \%$ more energy dissipation during indentation than the sample with 5 vol.\% $\mathrm{B}_{4} \mathrm{C}$ as the sintering additive resulting in a higher overall toughness.
\end{abstract}

Q2016 The Cerarric Society of Japan. AI rights reserved.

Key-words : Field-assisted sintering, Microstructure contrd, Dense ceramics, Sintering additives, Indentation

Received October 29, 2015; Accepted December 27, 2015]

\section{Introduction}

Ultra high temperature ceramics (UHTC) have generated a bt of interest as candidate materials for high temperature aerospace applications such as leading edges for hypersonic airerafts. Flight at hypersonic speeds generates enomous drag forces, which generates high temperatures at the shap leadings edges that are crucial to maintaining aerodynamic efficiency. UHTC (Group IV and $\mathrm{V}$ carbides and borides) have high melting po in $t=\left(>3000^{\circ} \mathrm{C}\right.$ ), making them ideal candidates for shamp leading edges and zero erosion throat inserts. Among the group V carbides, $\mathrm{TaC}$ has the highest melting point temperature of about $3950^{\circ} \mathrm{C}$ and $\mathrm{NbC}$ has the socond highest at about $3600^{\circ} \mathrm{C}$. ?)

The properties of UHTCs are highly influenced by the final grain size and morphology. According to the Hall-Petch relationship, the streng th increases as the inverse square of the grain size which is influenced by the starting powder size, sintering additives, and processing time and temperature. ${ }^{27}$ Because of their strong covalent bonds and the low self-diffusion coefficients of UHTCs, reaching full densification is a major challenge.

UHTCs such as $\mathrm{TaC}, \mathrm{NbC}, \mathrm{VC}$, and HfC, have been consolidated using conventional hot pressing, ${ }^{3,4}, 4$ pressureless sintering, 5,.9 vacuum plasma spraying," and high frequency induction heating. (8)-19) These methods only reach a densification of up to $97.5 \%$ for the case of $\mathrm{TaC}$ consolidation and is similar in consolidating other UHTCs. Consolidation by spark plasma sintering (SPS), however, has achieved full densification at lower temperatures $\left(1800-1900^{\circ} \mathrm{C}\right.$ for SPS comparod to $2100-2300^{\circ} \mathrm{C}$ for pressureless sintering and hot pressing) and shorter dwell times compared to other consolidation methods $(10 \mathrm{~min}$ hold time in SPS comparod to $30 \mathrm{~min}$ to several hours for pressureless sintering and hot pressing). ${ }^{11-144}$

Sintering aids such as carbon, $\mathrm{TaB}_{2}, \mathrm{TaSi}_{2}, \mathrm{MoSi}_{2}, \mathrm{SiC}, \mathrm{B}_{4} \mathrm{C}$, and $\mathrm{Si}$ have been utilized to improve the densification and

${ }^{1}$ Corresponding author: A. Agarwal; E-mail: agarwala $Q$ fiu.edu

$"$ Corresponding author: B. Boesl; E-mail: booesl@ fiu.edu

"t' Preface for this article: Dol http $/ /$ dxddoi.ong/10.2109/joersj2.124.P4-1 mechanical properties of the sintered ceramic., 3,4,100,14) The use of sintering aids can lead to the reduction in temperature when densification begins to take place. Not only will this lead to increased final densification, but it will also reduce the amount of grain growth occurrence. Liu et al. ${ }^{14)}$ consolidated a $\mathrm{TaC}-$ $20 \mathrm{vol} . \% \mathrm{SiC}$ composite using SPS and saw an increase in mechanical properties (Young's modulus, Vickers hardness, flexural strength, fracture toughness) that they attributed to a docrease in residual porosity.

In this study, a pre-alloyod powder of Tantalum-Niobium Carbide (TaC-NbC) made up of $80 \mathrm{wt}$. \% TaC and $20 \mathrm{wt} . \% \mathrm{NbC}$ was used. $\mathrm{NbC}$ has a density which is half that of $\mathrm{TaC}(7.78$ $\mathrm{g} / \mathrm{cm}^{3} \mathrm{vs} 14.5 \mathrm{~g} / \mathrm{cm}^{3}$, respoctively) and the addition of the 20 wt. \% NbC reduced the density of pre-alloyed composition to $12.36 \mathrm{~g} / \mathrm{cm}^{3}$.) There have boen previous studies on the sintering of $\mathrm{TaC}$ with and without the use of sintering additives, however the addition of $\mathrm{NbC}$ has not yet been studied. ${ }^{15-19}$ Because the properties of ultra high temperature ceramics make them candidate materials for high temperature space applications and rocket nozzle throat inserts, the high cost associated with send ing something into space or the increased payload capacity of a rocket as a result of a lower weight material being used can make these systems more viable and cost efficient.

The sintering additives investigated in this study $\left(\mathrm{B}_{4} \mathrm{C}\right.$ and $\left.\mathrm{Si}\right)$ were chosen based on their high temperature properties when considering the possible socondary phases formed during the sintering process ${ }^{2,20)}$ With the addition of $\mathrm{Si}$, the formation of $\mathrm{SiC}$ is expected which has a melting point of $2830^{\circ} \mathrm{C}$. With the addition of $\mathrm{B}_{4} \mathrm{C}, \mathrm{TaB}_{2}$ and $\mathrm{NbB}_{2}$ have the possibility to form which have melting points of 3037 and $3050^{\circ} \mathrm{C}$, respoctively. Hence, the novelty of this work is twofold; (1) the addition of $\mathrm{NbC}$ to $\mathrm{TaC}$ reduces the density of the system and (2) samples are sintered to $>99 \%$ relative density in as little as $3 \mathrm{~min}$ with improved toughness.

\section{Experimental procedure}

\subsection{Powder preparation}

Tantalum-Niobium Carbide powder (TaC20NbC; $80 \mathrm{wt} \%$ 


\title{
In Situ Mechanical Testing Techniques for Real-Time Materials Deformation Characterization
}

\author{
CHRIS RUDOLF, ${ }^{1}$ BENJAMIN BOESL, ${ }^{1}$ and ARVIND AGARWAL ${ }^{1,2}$ \\ 1.-Plasma Forming Laboratory, Composites Laboratory, Department of Mechanical and Mate- \\ rials Engineering, Florida International University, Miami, FL 33174, USA. 2.- e-mail: \\ agarwala@fiu.edu
}

In situ mechanical property testing has the ability to enhance quantitative characterization of materials by revealing the occurring deformation behavior in real time. This article will summarize select recent testing performed inside a scanning electron microscope on various materials including metals, ceramics, composites, coatings, and 3-Dimensional graphene foam. Tensile and indentation testing methods are outlined with case studies and preliminary data. The benefits of performing a novel double-torsion testing technique in situ are also proposed.

\section{INTRODUCTION}

In situ experimentation and materials processing has been carried out inside various tools including scanning electron microscopes (SEMs), transmission electron microscopes, $\mathrm{x}$-ray diffractometers, and ellipsometers. ${ }^{1-4}$ In situ experimentation has gained significant attention in recent years due to its ability to enhance material characterization. This is particularly true when performing mechanical testing inside a scanning electron microscope to determine fracture and failure mechanisms. In traditional testing, the point of failure is realized from the collected experimental data and possible fracture and cracking mechanisms can be obtained from post-experimental imaging. When performing the same tests inside of a scanning electron microscope with real-time imaging, elucidation of the specific deformation mechanisms that occur, as well as when they occur, allows for a better understanding of the material's properties and overall performance. With the recent advances in testing devices, many different materials, such as polymers, metals, ceramics, and carbon forms, can be characterized. In this research summary of in situ testing methods, tensile tests of a 3-Dimensional (3D) graphene foam, a metal composite with nanoscale reinforcement, and a metallic glassy coating, as well as an indentation test of a high-temperature ceramic, and a double-torsion testing technique for materials including brittle ceramics is summarized.

\section{TEST METHODS}

In situ tests are carried out using a linear, screwdriven micro-load frame (MTI Instruments SEM tester 1000) inside a dual-beam focused ion beam/ SEM (JEOL JIB-4500) (Fig. 1) under a vacuum atmosphere. The micro-load frame has a load capacity of $4500 \mathrm{~N}$ with an accuracy of $0.2 \%$ and a linear movement resolution of $20 \mathrm{~nm}$. The ability to change the attachment grips quickly makes running multiple tests possible. The load frame is controlled using an interface system that connects to the load frame by pass-through connections on the scanning electron microscope chamber. The software used to in put the test procedures and analyze and report the data is the MTEST Quattro system (ADMET, Norwood, MA). Testing procedures can be custom built, or preloaded test procedures that follow ASTM and ISO specifications can be chosen. Specimens to be tested must either be conductive or have a conductive coating applied in order to achieve quality imaging.

\section{In Situ Tensile Testing}

In situ tensile tests are performed by clamping a specimen into the micro-load frame by wedge grips on each side. Specimens of various sizes or dog-bone shapes can be used and input into the analysis software for accurate results. A main difficulty when performing in situ tensile testing is characterizing the material strain. Two methods have the potential to measure strain: both digital image 


\title{
In situ indentation behavior of bulk multi-layer graphene flakes with respect to orientation
}

\author{
Chris Rudolf, Benjamin Boesl, Arvind Agarwal * \\ Plasma Forming Labaratary, Composites Laborabry, Department of Mechanical and Materials Engineering Flarida Intemarianal University, 10555 West Flagler Street, Miami
} F. 33174 , United Srates

\section{A R T I C L. E I N F O}

\section{Artide histary:}

Received 23 February 2015

Received in revised form 21 July 2015

Accepted 22 July 2015

Available online 23 July 2015

\begin{abstract}
A B S T R A C T
In situ indentation is performed on bulk multi-layer graphene flakes (MLG) consolidated by spark plasma sintering to study the effect of orientation on the deformation behavior and associated energy dissipation capabilities of MLG. Spark plasma sintering of MLG aligns them into a uniformly oriented, densely packed pellet. With respect to the $2 \mathrm{D}$ surface of consolidated MLG, indentation is carried out on the surface (out-of-plane MLG orientation) and in the orthogonal direction (in-plane MLG orientation). The combination of instrumented indentation and imaging provided evidence of deformation and failure mechanisms in real-time, as well as a quantitative comparison of energy dissipation. Indentation performed in the orthogonal direction resulted in a work of indentation $270 \%$ greater than indentation performed on the surface. The prevalent energy dissipation mechanisms observed when indenting in the orthogonal direction are compressive reinforcement, bending. push-out, and pop-out while the prevalent mechanisms observed in the surface indent are sliding, bending, kinking, and MLG pull-out.

Q 2015 Elsevier Itd. All rights reserved.
\end{abstract}

\section{Introduction}

Graphene is a single layer of $\mathrm{sp}^{2}$ bonded carbon that is known for its excellent thermal, mechanical, and electrical properties. The functional properties of graphene include having a high Young's modulus (0.5-1 TPa) [1] and high tensile strength (130 GPa) [2], which has gained it considerable attention for use as reinforcement in polymer, metallic, and ceramic composite matrices [3-5]. Rafiee et al. [3] found that compared to other carbon-based nanostructures, such as single- and multi-walled carbon nanotubes, a low content of graphene addition offered better improvement in mechanical properties in a reinforced epoxy nanocomposite. While offering great reinforcement properties, the use of graphene was slow until the development of multi-layer graphene flakes (MLG) which are also called graphene nanosheets [6]. Multi-layer graphene flakes are particles consisting of multiple layers of stacked graphene [7]. MLG are becoming more widely used because they are easier and less expensive to form compared to single layer graphene while retaining much of the desirable properties $[8,9]$. Typically, MLG are made up of $10-30$ sheets of graphene held together by weak van der Waals forces and have a thickness of 3-10 nm and a width of $1-25 \mu \mathrm{m}$, providing large surface areas.

\footnotetext{
* Corresponding author.

E-mail address: agarwa laøfiuedu (A. Agarwal)
}

The mechanical properties of graphene have been previously reported by Lee et al. [2] who performed atomic force microscopy on a suspended graphene flake and found that the bending rigidity is higher in the principal (in-plane) direction. Golkarian et al. [10] reported on the effect of the van der Waals forces when increasing the number of layers in graphite flakes and found by theoretical modeling that the Young's modulus decreased about 13\% with increasing the number of layers fourfold. Nanoindentation, previously performed on fully dense samples of MLG consolidated by spark plasma sintering (SPS), showed a correlation of mechanical properties as a function of loading direction [11]. Energy dissipation mechanisms were observed post-fracture with a goal of evaluating future use of MLG in ceramic composites. Subsequent published reports on MLG-reinforced composites detail the various deformation or energy dissipation mechanisms. Nieto et al. [12] prepared a 5 vol.\% graphene platelet reinforced tantalum carbide composite by spark plasma sintering and reported a fracture toughness increase of $99 \%$ over the monolith. Additionally, post fracture analysis of the graphene platelet fracture surfaces has shown evidence of energy dissipation mechanisms [6]. Hypotheses for increased toughness in composites reinforced with MLG can be attributed to three different regimes; (i) property changes during processing, (ii) increased load capacity prior to initial crack propagation, and (iii) crack propagation suppression mechanisms, examples of toughening mechanisms occurring in regime (i) include increased densification and grain wrapping 
VITA

\section{CHRISTOPHER RUDOLF}

\section{EDUCATION AND EXPERIENCE:}

Sept. 2007 - May 2012 Bachelor of Science (B.S.) in Mechanical Engineering, Florida State University, Tallahassee, FL

Sept. 2012 - Dec. 2013 Master of Science (M.S.) in Mechanical Engineering, Florida International University, Miami, FL

Jan. 2014 - Aug. 2016 Doctoral Candidate (Ph.D) in Mechanical Engineering, Florida International University, Miami, FL

Jan. 2013 - May 2014 Teaching Assistant, Department of Mechanical and Materials Engineering, Florida International University

Jan. 2014 - Aug. 2016

Research Assistant, Department of Mechanical and Materials Engineering, Florida International University

\section{PUBLICATIONS AND PRESENTATIONS:}

1. P. Nautiyal, Chris Rudolf, A. Loganathan, C. Zhang, B. Boesl, A. Agarwal. Directionally Aligned Ultra-long Boron Nitride Nanotube Induced Strengthening of Aluminum based Sandwich Composite. Adv. Eng. Mater. (Online: 20 July 2016)

2. Christopher C. Rudolf, B. Boesl, A. Agarwal. TaC-NbC Formed by Spark Plasma Sintering with the Addition of Sintering Additives. J. Ceram. Soc. Japan 124 (4) (2016) 381-387.

3. M. Asadikiya, Christopher Rudolf, C. Zhang, B. Boesl, Y. Zhong. The Role of CALPHAD Approach in the Sintering of B4C with SiC as a Sintering Aid by Spark Plasma Sintering Technique. 11th International Conference on Ceramic Materials \& Components for Energy \& Environmental Applications, February 2016.

4. Chris Rudolf, B. Boesl, A. Agarwal. In Situ Mechanical Testing Techniques for RealTime Materials Deformation Characterization. JOM 68(1) (2016) 136-142.

5. Chris Rudolf, B. Boesl, A. Agarwal. In situ indentation behavior of bulk multi-layer graphene flakes with respect to orientation. Carbon 94 (2015) 872-878.

6. Christopher Rudolf. TaCNbC Formed by Spark Plasma Sintering with the Addition of Sintering Additives. Presented in the Sintering and Related Powder Processing Science and Technologies symposium at the Materials Science \& Technology 2015 conference, Columbus, $\mathrm{OH}$.

7. Christopher Rudolf. Ultrahigh Temperature Ceramics (UHTC) Materials with NanoReinforcement. Presented at the 2015 AFRL Research Collaboration Program Review, Dayton, $\mathrm{OH}$. 